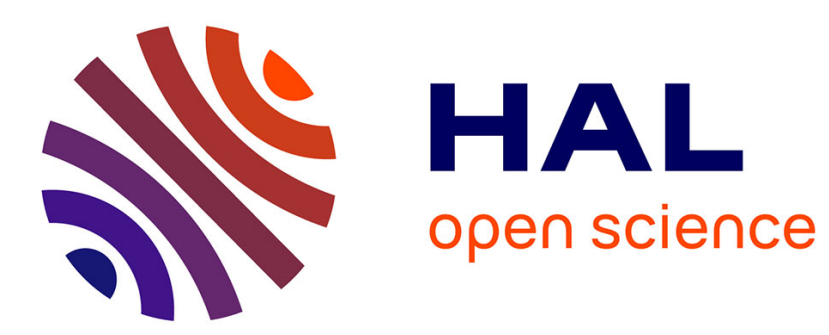

\title{
Grade filtration of linear functional systems
}

Alban Quadrat

\section{To cite this version:}

Alban Quadrat. Grade filtration of linear functional systems. [Research Report] RR-7769, INRIA. 2011, pp.86. inria-00632281

\section{HAL Id: inria-00632281 https://hal.inria.fr/inria-00632281}

Submitted on 14 Oct 2011

HAL is a multi-disciplinary open access archive for the deposit and dissemination of scientific research documents, whether they are published or not. The documents may come from teaching and research institutions in France or abroad, or from public or private research centers.
L'archive ouverte pluridisciplinaire HAL, est destinée au dépôt et à la diffusion de documents scientifiques de niveau recherche, publiés ou non, émanant des établissements d'enseignement et de recherche français ou étrangers, des laboratoires publics ou privés. 


\section{N R I A}

INSTITUT NATIONAL DE RECHERCHE EN INFORMATIQUE ET EN AUTOMATIQUE

\section{Grade filtration of linear functional systems}

Alban Quadrat

\section{$\mathbf{N}^{\circ} \mathbf{7 7 6 9}$}

October 2011

Modeling, Optimization, and Control of Dynamic Systems 



\title{
Grade filtration of linear functional systems
}

\author{
Alban Quadrat* \\ Theme : Modeling, Optimization, and Control of Dynamic Systems \\ Équipe-Projet DISCO
}

Rapport de recherche $\mathrm{n}^{\circ} 7769$ - October 2011 - 86 pages

\begin{abstract}
The grade filtration of a finitely generated left module $M$ over an Auslander regular ring $D$ is a built-in classification of the elements of $M$ in terms of their grades (or their (co)dimensions if $D$ is also a Cohen-Macaulay ring). In this paper, we show how grade filtration can be explicitly characterized by means of elementary methods of homological algebra. Our approach avoids the use of sophisticated methods such as bidualizing complexes, spectral sequences, associated cohomology, and Spencer cohomology used in the literature of algebraic analysis. Efficient implementations dedicated to the computation of grade filtration can then be easily developed in the standard computer algebra systems (see the Maple package PURITYFiLtration and the GAP4 package AbelianSystems). Moreover, this characterization of grade filtration is shown to induce a new presentation of the left $D$-module $M$ which is defined by a block-triangular matrix formed by equidimensional diagonal blocks. The linear functional system associated with the left $D$-module $M$ can then be integrated in cascade by successively solving inhomogeneous linear functional systems defined by equidimensional homogeneous linear systems of increasing dimension. This equivalent linear system generally simplifies the computation of closed-form solutions of the original linear system. In particular, many classes of underdetermined/overdetermined linear systems of partial differential equations can be explicitly integrated by the packages PURITYFILTRATION and AbelianSystems, but not by computer algebra systems such as Maple.
\end{abstract}

Key-words: Algebraic analysis, grade filtration, module theory, homological algebra, symbolic computation, mathematical systems theory, underdetermined/overdetermined linear functional systems, linear systems of partial differential equations.

\footnotetext{
* INRIA Saclay - Île-de-France, DISCO project, L2S, Supélec, 3 rue Joliot Curie, 91192 Gif-sur-Yvette Cedex, France, alban.quadrat@inria.fr, http://www.sophia.inria.fr/members/Alban.Quadrat/index.html.
} 


\section{Filtration par grade des systèmes linéaires fonctionnels}

Résumé : La filtration par grade d'un module à gauche $M$ finiment engendré sur un anneau Auslander-régulier $D$ est une classification intrinsèque des éléments de $M$ en fonction de leurs grades (ou de leurs (co)dimensions si $D$ est aussi un anneau de Cohen-Macaulay). Dans ce papier, nous montrons comment la filtration par grade peut être explicitement caractérisée au moyen de techniques élémentaires d'algèbre homologique. Notre approche évite l'utilisation de techniques sophistiquées telles que les complexes bidualisants, les suites spectrales, la cohomologie associée et la cohomologie de Spencer utilisées dans la littérature d'analyse algébrique. Des implantations efficaces dédiées au calcul de la filtration par grade peuvent alors être facilement développées dans les systèmes standards de calcul formel (voir le package PURityFiLtration de Maple et le package AbelianSystems de GAP4). De plus, cette caractérisation de la filtration par grade induit une nouvelle présentation du $D$-module à gauche $M$ qui est définie par une matrice triangulaire par blocs formée de blocs diagonaux équidimensionnels. Le système linéaire fonctionnel associé au $D$-module à gauche $M$ peut alors être intégré en cascade par la résolution successive de systèmes linéaires fonctionnels inhomogènes définis par des systèmes linéaires homogènes équidimensionnels de dimension croissante. Ce système linéaire équivalent simplifie généralement le calcul des solutions sous formes closes du système linéaire originel. En particulier, de nombreux systèmes linéaires sur-déterminés/sous-déterminés d'équations aux dérivées partielles peuvent être explicitement intégrés au moyen des packages PURITYFILTRATION et AbelianSystems, alors qu'ils ne peuvent l'être par des systèmes de calcul formel tels que Maple.

Mots-clés : Analyse algébrique, filtration par grade, théorie des modules, algèbre homologique, calcul formel, théorie mathématique des systèmes, systèmes linéaires fonctionnels sur-déterminés/ sous-déterminés, systèmes linéaires d'équations aux dérivées partielles. 


\section{Introduction}

The theory of linear functional systems such as linear systems of partial differential/time-delay/ difference equations is a rich branch of mathematics which finds its foundation in mathematical physics. Different analytic methods can be used to study determined linear functional systems (see, e.g., [19]), namely linear functional systems containing as many unknown functions as functionally independent linear equations. Overdetermined (resp., underdetermined) linear functional systems, namely linear functional systems containing fewer (resp., more) unknown functions than functionally independent linear equations, also find important applications in mathematical physics (see, e.g., [13, 38]), in differential geometry (see, e.g., [24, 38]), or in mathematical systems theory (see, e.g., [14, 36, 38, 40]). Formal methods for studying overdetermined linear systems of PD equations can be traced back to the works of Cartan, Riquier and Janet [27]. A modern approach was developed in the sixties by Spencer and his collaborators (see, e.g., [38, 51]). Gröbner bases and Janet bases [12, 27] over a noncommutative polynomial ring of functional operators are nowadays two fundamental computational tools for the formal study of overdetermined linear functional systems (see, e.g., [14, 31, 48]).

Despite these important computational methods, computer algebra systems still have many difficulties to find closed-form solutions of overdetermined or undetermined linear functional systems (when they exist), for instance of linear systems of PD equations. One of the main reasons for this failure is that linear functional systems generally mix together unknown functions which satisfy linear functional systems of different dimension. For instance, the integration of the unknown functions of an overdetermined linear systems of PD equations depends on arbitrary functions of a certain number of the independent variables (due to the Cartan-Kähler-Janet theorem which generalizes the well-known Cauchy-Kowalevski theorem) (see, e.g., [27, 38, 51]). The maximal number of independent variables which appear in these arbitrary functions (sometimes plus the number of independent variables) is called the dimension of the system. Hence, an important issue for the study of overdetermined linear functional systems is to determine the unknown functions or their linear functional combinations which satisfy a linear functional system of a given dimension. This problem, related to the equidimensional decomposition of algebraic varieties (see, e.g., [20, 25, 49]), has lengthly been studied within algebraic analysis and algebraic/analytic D-module theory [9, 10, 11, 33] by Roos [49, Sato and Kashiwara [29, 30, Björk [9, 10], Ginsburg [23], and others. This problem corresponds to the so-called grade filtration $\left\{M_{i}\right\}_{i \geq 0}$ (also called bidualizing or purity filtration) of the finitely generated left $D$-module $M$ which defines the linear system of PD equations, where $D$ is a noncommutative polynomial ring of PD operators satisfying certain regularity conditions (e.g., $D$ is an Auslander regular ring). This filtration of $M$ is defined by the left $D$-submodules $M_{i}$ 's of $M$ formed by the elements of $M$ having a codimension (or a grade) greater or equal to $i$. The existence of the grade filtration of a finitely generated left/right module $M$ over an Auslander regular ring $D$ is proved in [9, 10, 23, 32, 49] (resp., in [30, 29]) using bidualizing complexes and spectral sequence arguments (resp., derived categories, derived functors and associated cohomology [25]), i.e., by means of sophisticated homological algebra techniques (resp., modern developments of category theory). See also [38, 39] (resp., [37]) for a recent study of grade filtration based on Spencer cohomology and Spencer sequences (resp., Gabriel localization for commutative polynomial rings). Despite the difficulties for the computation of the spectral sequences defining the grade filtration, they were recently made constructive in [2, 3] thanks to the new concept of generalized morphisms, and they were implemented in the homalg package [8] of the system GAP4 [22] (homalg is a package dedicated to homological algebra oriented computations). To our knowledge, it is the first implementation of the computation of the grade filtration in a computer algebra system. 
We refer the reader to [20, 25, 49] (resp., [9, 10, 23, 29]) for applications of grade filtration to algebraic geometry (resp., algebraic analysis). Finally, techniques based on grade filtration have recently been introduced in mathematical systems theory (see [4, 37, 38, 39, 40, 41, 42, 43, 44]).

The purpose of this paper is to develop a new algorithm which computes the grade filtration of a finitely generated left module $M$ over a noetherian regular domain $D$ satisfying a slightly weaker condition than the standard Auslander condition (see, e.g., [9, 10]). In particular, many important classes of noncommutative polynomial rings of functional systems satisfy these conditions. The first benefit of this new algorithm is that it is an extension of the methods developed in [1, 14, 30, 38, 40] for the classification of modules (torsion modules, modules with torsion submodules, torsion-free/reflexive/projective modules). These methods have recently been applied to solve the problem of parametrizing underdetermined linear functional systems by means of arbitrary functions (potentials) studied in mathematical physics and in control theory (see [14, 15, 21, 38, 40, 53]). The second benefit of this algorithm is that it is conceptually much simpler than the algorithms based on bidualizing complexes, spectral sequences and associated cohomology. In particular, it can be easily implemented in any computer algebra system in which Gröbner basis techniques are available (e.g., Maple, Mathematica, Singular, Macaulay2, Magma). The corresponding algorithm was implemented by the author in the Maple package PurityFiltration [45] built upon Oremodules [15]. Using the PurityFiltration package, classes of overdetermined/underdetermined linear systems of PD equations which cannot be directly integrated by Maple can be explicitly solved [45] (see also the forthcoming homalg based package D-modules). Moreover, the algorithm has also been implemented recently in the homalg project package AbelianSystems [7] developed in collaboration with M. Barakat (University of Kaiserslautern). This implementation is much faster than the original homalg command based on spectral sequence computation (10 times faster on small PD examples), and thus it can be used to study larger examples. We hope that the results developed in this paper and demonstrated by the PURITYFiltration and AbelianSystems packages will be used in the future to improve standard computer algebra systems such as Maple or Mathematica for the symbolic integration of overdetermined/underdetermined linear functional systems. More generally, this new algorithm holds for constructive abelian categories [6], and thus it can be used in different contexts such as the computation of the grade filtration of coherent sheaves over projective schemes as shown in the homalg project package Sheaves [5].

Since techniques of module theory, homological algebra and algebraic analysis are not largely well-known, they are summarized in Section 2, The main results about grade filtration are developed in Section 3. In Section 4, we show how the concept of grade filtration can be used to compute an equivalent block-triangular form of a linear functional system whose diagonal blocks define equidimensional linear functional systems. The integration of the original system is then equivalent to a cascade integration of inhomogeneous linear functional systems, the corresponding homogeneous linear systems being equidimensional and of increasing dimension (e.g., we first integrate a 0-dimensional/holonomic homogenous linear system, then an inhomogeneous linear systems defined by a 1-dimensional/subholonomic homogeneous linear system, ...). In Section 5. we briefly give a few extensions of the results obtained in Section 3 . Finally, in Appendix, we demonstrate the PURITYFiltration package through different explicit examples.

\section{Algebraic analysis approach to linear functional systems}

In what follows, $D$ will always be a noetherian ring, i.e., a ring $D$ that is both a left and a right noetherian ring (see, e.g., [50]). Moreover, the set of $q \times p$ matrices with entries in $D$ is 
denoted by $D^{q \times p}$ and the unit of the ring $D^{p \times p}$ by $I_{p}$. If $\mathcal{F}$ is a left $D$-module (e.g., $\mathcal{F}=D$ ) and $R \in D^{q \times p}$, then.$R$ and $R$. are respectively the left $D$-homomorphism (i.e., the left $D$-linear map) and the abelian group homomorphism (i.e., $\mathbb{Z}$-homomorphism) defined by:

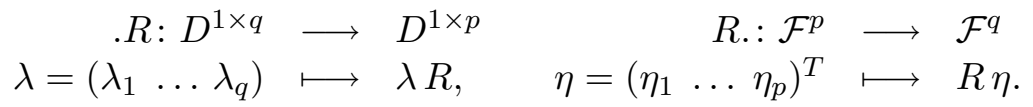

With the above notations, we call linear system an abelian group of the form:

$$
\operatorname{ker}_{\mathcal{F}}(R .)=\left\{\eta \in \mathcal{F}^{p} \mid R \eta=0\right\} .
$$

The study of $\operatorname{ker}_{\mathcal{F}}(R$. $)$ in terms of the finitely presented left $D$-module $M=D^{1 \times p} /\left(D^{1 \times q} R\right)$ and of the left $D$-module $\mathcal{F}$ was first developed in [34]. This idea is nowadays the cornerstone of the algebraic D-module theory (or algebraic analysis), developed by Bernstein and Sato's school (particularly by Kashiwara), in which $D$ stands for a noncommutative ring of partial differential (PD) operators with coefficients in a differential ring (see, e.g., 9, 10, 11, 30, 33]). More precisely, if $A$ is a ring and $\left\{\delta_{i}\right\}_{i=1, \ldots, n}$ are $n$ commuting derivations of $A$, namely, $\delta_{i}: A \longrightarrow A$ satisfies $\delta_{i}\left(a_{1}+a_{2}\right)=\delta_{i}\left(a_{1}\right)+\delta_{i}\left(a_{2}\right), \delta_{i}\left(a_{1} a_{2}\right)=\delta_{i}\left(a_{1}\right) a_{2}+a_{1} \delta_{i}\left(a_{2}\right)$ for all $a_{1}, a_{2} \in A$ and for all $i=1, \ldots, n$, and $\delta_{i} \circ \delta_{j}=\delta_{j} \circ \delta_{i}$ for all $i, j=1, \ldots, n$, then the ring $D=A\left\langle\partial_{1}, \ldots, \partial_{n}\right\rangle$ of PD operators with coefficients in $A$ is the noncommutative polynomial ring in $\partial_{1}, \ldots, \partial_{n}$ which satisfies the relations $\partial_{i} a=a \partial_{i}+\delta_{i}(a)$ for all $a \in A$ and for all $i=1, \ldots, n$, and $\partial_{i} \partial_{j}=\partial_{j} \partial_{i}$ for all $i, j=1, \ldots, n$. Prototype examples of a ring $D$ of PD operators are the so-called $W e y l$ algebras $A_{n}(k)$ and $B_{n}(k)$ of $\mathrm{PD}$ operators with respectively coefficients in $A=k\left[x_{1}, \ldots, x_{n}\right]$ and in $A=k\left(x_{1}, \ldots, x_{n}\right)$, where $k$ is a field (that we shall suppose to be of characteristic 0 ), $\hat{\mathcal{D}}_{n}(k)$, or $\mathcal{D}_{n}\left(k^{\prime}\right)$ the rings of PD operators with coefficients in the ring of formal power series $A=k \llbracket x_{1}, \ldots, x_{n} \rrbracket$ or in the ring of locally convergent power series $A=k^{\prime}\left\{x_{1}, \ldots, x_{n}\right\}$, where $k^{\prime}=\mathbb{R}$ or $\mathbb{C}$. These rings are noetherian domains (see, e.g., [9, 11, 33]). If $D$ is a ring of PD operators and $\mathcal{F}$ a left $D$-module (e.g., $\mathcal{F}=A$ ), then $R \in D^{q \times p}$ is a matrix of PD operators and the linear system $\operatorname{ker}_{\mathcal{F}}(R$.) is the $k$-vector space formed by the $\mathcal{F}$-solutions of the linear system of $\mathrm{PD}$ equations $R \eta=0$. Within algebraic analysis, more general classes of noncommutative polynomial rings of functional operators can be considered such as Ore algebras as explained in [14], which allows one to consider a more general class of linear functional systems.

Let us now explain basic ideas of algebraic analysis. Let $\pi: D^{1 \times p} \longrightarrow M$ be the left $D$ homomorphism which maps $\lambda \in D^{1 \times q}$ to its residue class $\pi(\lambda) \in M$, and $\left\{f_{j}\right\}_{j=1, \ldots, p}$ the standard basis of $D^{1 \times p}$, namely, $f_{j}$ is the row vector of length $p$ with 1 at the $j^{\text {th }}$ position and 0 elsewhere. Then, $\left\{y_{j}=\pi\left(f_{j}\right)\right\}_{j=1, \ldots, n}$ is a family of generators of $M$ since for every $m \in M$, there exists $\lambda=\left(\lambda_{1} \ldots \lambda_{p}\right) \in D^{1 \times p}$ such that $m=\pi(\lambda)$, which yields:

$$
m=\pi(\lambda)=\pi\left(\sum_{j=1}^{p} \lambda_{j} f_{j}\right)=\sum_{j=1}^{p} \lambda_{j} \pi\left(f_{j}\right)=\sum_{j=1}^{p} \lambda_{j} y_{j} .
$$

The family of generators $\left\{y_{j}\right\}_{j=1, \ldots, p}$ of $M$ satisfies $D$-linear relations: if $R_{i}$ denotes the $i^{\text {th }}$ row of $R$, then $R_{i \bullet} \in D^{1 \times q} R$, which yields $\pi\left(R_{i \bullet}\right)=0$, and thus:

$$
\forall i=1, \ldots, q, \quad \pi\left(R_{i \bullet}\right)=\pi\left(\sum_{j=1}^{p} R_{i j} f_{j}\right)=\sum_{j=1}^{p} R_{i j} \pi\left(f_{j}\right)=\sum_{j=1}^{p} R_{i j} y_{j}=0 .
$$

If $y=\left(y_{1} \ldots y_{p}\right)^{T} \in M^{p}$, then the above relations can be rewritten as $R y=0$. 
Now, if $\mathcal{F}$ is a left $D$-module, $\operatorname{hom}_{D}(M, \mathcal{F})$ the abelian group of left $D$-homomorphisms from $M$ to $\mathcal{F}$, and $\phi \in \operatorname{hom}_{D}(M, \mathcal{F})$, then $\eta=\left(\phi\left(y_{1}\right) \ldots \phi\left(y_{p}\right)\right)^{T} \in \mathcal{F}^{p}$ and

$$
\forall i=1, \ldots, q, \quad \sum_{j=1}^{p} R_{i j} \eta_{j}=\sum_{j=1}^{p} R_{i j} \phi\left(y_{j}\right)=\phi\left(\sum_{j=1}^{p} R_{i j} y_{j}\right)=\phi(0)=0,
$$

i.e., $\eta \in \operatorname{ker}_{\mathcal{F}}\left(R\right.$.). Conversely, if $\eta \in \operatorname{ker}_{\mathcal{F}}\left(R\right.$.), then we can define the map $\phi_{\eta}: M \longrightarrow \mathcal{F}$ by $\phi_{\eta}(\pi(\lambda))=\lambda \eta$ for all $\lambda \in D^{1 \times p}$. Indeed, $\phi_{\eta}$ is well-defined: if $\pi(\lambda)=\pi\left(\lambda^{\prime}\right)$, then $\lambda=\lambda^{\prime}+\mu R$, for a certain $\mu \in D^{1 \times q}$, which yields $\phi_{\eta}(\pi(\lambda))=\lambda \eta=\lambda^{\prime} \eta+\mu R \eta=\lambda^{\prime} \eta$. The map $\phi_{\eta}$ is clearly left $D$-linear and $\phi_{\eta}(0)=0$ since $\phi_{\eta}\left(\sum_{j=1}^{p} R_{i j} y_{j}\right)=\sum_{j=1}^{p} R_{i j} \eta_{j}=0$ for all $i=1, \ldots, q$, and thus $\phi_{\eta} \in \operatorname{hom}_{D}(M, \mathcal{F})$. If we introduce the following abelian group homomorphisms

$$
\begin{aligned}
\sigma: \operatorname{ker}_{\mathcal{F}}(R .) & \longrightarrow \operatorname{hom}_{D}(M, \mathcal{F}) \quad \chi: \operatorname{hom}_{D}(M, \mathcal{F}) \\
\eta & \longrightarrow \phi_{\eta},
\end{aligned}
$$

then $\chi \circ \sigma=\operatorname{id}_{\operatorname{ker}_{\mathcal{F}}(R .)}$ since $\phi_{\eta}\left(y_{j}\right)=\eta_{j}$ for all $j=1, \ldots, p$, and $\sigma \circ \chi=\operatorname{id}_{\operatorname{hom}_{D}(M, \mathcal{F})}$ since $(\sigma \circ \chi)(\phi)=\phi_{\left(\phi\left(y_{1}\right) \ldots \phi\left(y_{p}\right)\right)^{T}}=\phi$, which shows that $\chi^{-1}=\sigma$, and proves that $\operatorname{ker}_{\mathcal{F}}(R$. $)$ and $\operatorname{hom}_{D}(M, \mathcal{F})$ are isomorphic as abelian groups, which is denoted by $\operatorname{ker}_{\mathcal{F}}(R$. $) \cong \operatorname{hom}_{D}(M, \mathcal{F})$.

Theorem 1 ([34]). With the previous notations, we have:

$$
\operatorname{ker}_{\mathcal{F}}(R .) \cong \operatorname{hom}_{D}(M, \mathcal{F}) .
$$

Theorem 1 shows that the linear system $\operatorname{ker}_{\mathcal{F}}(R$. $)$ can be intrinsically studied by means of the two left $D$-modules $M=D^{1 \times p} /\left(D^{1 \times q} R\right)$ and $\mathcal{F}$. The matrix $R$ is a particular finite presentation of the left $D$-module $M$ defined up to isomorphism (see, e.g., [50]). Hence, we can study the solution space $\operatorname{hom}_{D}(M, \mathcal{F})$ independently of the particular embedding of $\operatorname{ker}_{\mathcal{F}}\left(R\right.$.) into $\mathcal{F}^{p}$. A second benefit of Theorem 1 is that the linear system $\operatorname{ker}_{\mathcal{F}}(R$. $)$ can be studied by means of the properties of the left $D$-modules $M$ and $\mathcal{F}$.

Definition 1 ([50]). Let $D$ be a noetherian ring and $M$ a finitely generated left $D$-module.

1. $M$ is free if there exists $r \in \mathbb{N}=\{0,1,2, \ldots\}$ such that $M \cong D^{1 \times r}$. Then, $r$ is then called the rank of $M$.

2. $M$ is projective if there exist $r \in \mathbb{N}$ and a left $D$-module $N$ such that $M \oplus N \cong D^{1 \times r}$, where $\oplus$ denotes the direct sum of left $D$-modules.

3. $M$ is reflexive if the left $D$-homomorphism $\varepsilon: M \longrightarrow \operatorname{hom}_{D}\left(\operatorname{hom}_{D}(M, D), D\right)$, defined by $\varepsilon(m)(f)=f(m)$ for all $m \in M$ and for all $f \in \operatorname{hom}_{D}(M, D)$, is an isomorphism.

4. If $D$ is a domain, then $M$ is torsion-free if the torsion left $D$-submodule of $M$ defined by

$$
t(M)=\{m \in M \mid \exists d \in D \backslash\{0\}: d m=0\}
$$

is reduced to 0 , i.e., if $t(M)=0$.

5. If $D$ is a domain, then $M$ is torsion if $t(M)=M$, i.e., if every element of $M$ is a torsion element.

Theorem 2 ([50]). A free module is projective, a projective module is reflexive, and a reflexive module is torsion-free.

In the next sections, we summarize basic homological techniques which will be used to algorithmically test whether or not $M$ admits torsion elements or is torsion-free, reflexive or projective (see Theorem 5 thereafter). These techniques will then be generalized in Section 3 to obtain an explicit characterization of the so-called grade filtration of $M$. 


\subsection{Basic homological algebra}

Let us shortly recall a few definitions of homological algebra (see, e.g., [50]).

Definition 2. 1. A complex, denoted by

$$
M_{\bullet} \ldots \stackrel{d_{i+2}}{\longrightarrow} M_{i+1} \stackrel{d_{i+1}}{\longrightarrow} M_{i} \stackrel{d_{i}}{\longrightarrow} M_{i-1} \stackrel{d_{i-1}}{\longrightarrow} \ldots,
$$

is a sequence of left (resp., right) $D$-modules $M_{i}$ and of left (resp., right) $D$-homomorphisms $d_{i}: M_{i} \longrightarrow M_{i-1}$ that satisfy $\operatorname{im} d_{i+1} \subseteq \operatorname{ker} d_{i}$, i.e., $d_{i} \circ d_{i+1}=0$ for all $i \in \mathbb{Z}$.

2. The defect of exactness of (1) at $M_{i}$ is the left (resp., right) $D$-module defined by:

$$
H_{i}\left(M_{\bullet}\right) \triangleq \operatorname{ker} d_{i} / \operatorname{im} d_{i+1} .
$$

3. The complex (1) is exact at $M_{i}$ if $H_{i}\left(M_{\bullet}\right)=0$, i.e., if ker $d_{i}=\operatorname{im} d_{i+1}$, and exact if ker $d_{i}=\operatorname{im} d_{i+1}$ for all $i \in \mathbb{Z}$. An exact complex is called an exact sequence.

4. An exact sequence of the form

$$
0 \longrightarrow M^{\prime} \stackrel{f}{\longrightarrow} M \stackrel{g}{\longrightarrow} M^{\prime \prime} \longrightarrow 0,
$$

i.e., $f$ is injective, $\operatorname{ker} g=\operatorname{im} f$ and $g$ is surjective, is called a short exact sequence.

5. A projective resolution of a left $D$-module $M$ is an exact sequence of the form

$$
\ldots \stackrel{d_{4}}{\longrightarrow} P_{3} \stackrel{d_{3}}{\longrightarrow} P_{2} \stackrel{d_{2}}{\longrightarrow} P_{1} \stackrel{d_{1}}{\longrightarrow} P_{0} \stackrel{d_{0}}{\longrightarrow} M \longrightarrow 0,
$$

where the $P_{i}$ 's are projective left $D$-modules and $d_{i} \in \operatorname{hom}_{D}\left(P_{i}, P_{i-1}\right)$ for all $i \in \mathbb{N}$. The smallest $n \in \mathbb{N}$ such that $P_{m}=0$ for all $m>n$ is called the length of the projective resolution of $M$. Similarly for right $D$-modules.

6. A free resolution of a left $D$-module $M$ is an exact sequence of the form

$$
\ldots \stackrel{. R_{3}}{\longrightarrow} D^{1 \times p_{2}} \stackrel{. R_{2}}{\longrightarrow} D^{1 \times p_{1}} \stackrel{. R_{1}}{\longrightarrow} D^{1 \times p_{0}} \stackrel{\pi}{\longrightarrow} M \longrightarrow 0,
$$

where $R_{i} \in D^{p_{i} \times p_{i-1}}$ and $. R_{i}: D^{1 \times p_{i}} \longrightarrow D^{1 \times p_{i-1}}$ is defined by $\left(. R_{i}\right)(\lambda)=\lambda R_{i}$.

7. A free resolution of a right $D$-module $N$ is an exact sequence of the form

$$
0 \longleftarrow N \stackrel{\kappa}{\longleftarrow} D^{q_{0}} \stackrel{S_{1} .}{\longleftarrow} D^{q_{1}} \stackrel{S_{2} .}{\longleftarrow} D^{q_{2}} \stackrel{S_{3} .}{\longleftarrow} \ldots,
$$

where $S_{i} \in D^{q_{i-1} \times q_{i}}$ and $S_{i}: D^{q_{i}} \longrightarrow D^{q_{i-1}}$ is defined by $\left(S_{i} \cdot\right)(\eta)=S_{i} \eta$.

Example 1. If $D$ is a noetherian domain and $M$ a finitely generated left $D$-module, then we have the following short exact sequence of left $D$-modules:

$$
0 \longrightarrow t(M) \stackrel{j}{\longrightarrow} M \stackrel{\rho}{\longrightarrow} M / t(M) \longrightarrow 0 .
$$

Remark 1. A module $M$ is not defined by a unique projective/free resolution: Fitting's lemma asserts that if $0 \longrightarrow \operatorname{ker} \pi \longrightarrow P \stackrel{\pi}{\longrightarrow} M \longrightarrow 0$ and $0 \longrightarrow \operatorname{ker} \pi^{\prime} \longrightarrow P^{\prime} \stackrel{\pi^{\prime}}{\longrightarrow} M \longrightarrow 0$ are two exact sequences, where $P$ and $P^{\prime}$ are projective/free modules, then $\operatorname{ker} \pi \oplus P^{\prime} \cong \operatorname{ker} \pi^{\prime} \oplus P$ (see, e.g., [50]). This isomorphism does not generally imply that $\operatorname{ker} \pi \cong \operatorname{ker} \pi^{\prime}$. We say that ker $\pi$ depends on $M$ up to a projective equivalence (see, e.g., [50]). Similarly, if we consider two

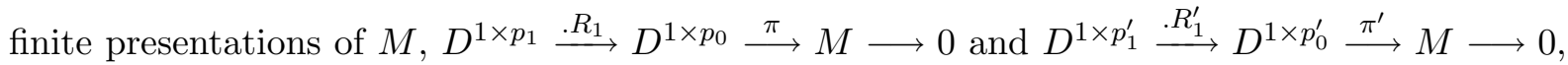
then $\operatorname{ker}_{D}\left(. R_{1}\right) \oplus D^{1 \times\left(p_{1}^{\prime}+p_{0}\right)} \cong \operatorname{ker}_{D}\left(. R_{1}^{\prime}\right) \oplus D^{1 \times\left(p_{1}+p_{0}^{\prime}\right)}$. For more details, see, e.g., [50]. For a constructive proof, see [18]. Similar results hold for all the syzygy modules $\operatorname{ker}_{D}\left(. R_{i}\right)$ 's of $M$. 
Since $D$ is a noetherian ring, one can easily prove that every finitely generated left (resp. right) $D$-module $M$ admits a free resolution (see, e.g., [50]). Now, if $\mathcal{F}$ is a left $D$-module, then using a free resolution (3) of a finitely generated left $D$-module $M$, we can define the extension abelian groups $\operatorname{ext}_{D}^{i}(M, \mathcal{F})$ 's for $i \geq 0$ as follows. Up to abelian group isomorphism, they are defined by the defects of exactness of the following complex of abelian groups

$$
\ldots \stackrel{R_{i+1} \cdot}{\longleftarrow} \mathcal{F}^{p_{i}} \stackrel{R_{i} \cdot}{\longleftarrow} \mathcal{F}^{p_{i-1}} \stackrel{R_{i-1}}{\longleftarrow} \ldots \stackrel{R_{3} \cdot}{\longleftarrow} \mathcal{F}^{p_{2}} \stackrel{R_{2} \cdot}{\longleftarrow} \mathcal{F}^{p_{1}} \stackrel{R_{1} \cdot}{\longleftarrow} \mathcal{F}^{p_{0}} \longleftarrow 0,
$$

where $R_{i}:: \mathcal{F}^{p_{i-1}} \longrightarrow \mathcal{F}^{p_{i}}$ is defined by $\left(R_{i}.\right)(\eta)=R_{i} \eta$ for all $\eta \in \mathcal{F}^{p_{i-1}}$ and $i \geq 1$, namely:

$$
\left\{\begin{array}{l}
\operatorname{ext}_{D}^{0}(M, \mathcal{F}) \cong \operatorname{ker}_{\mathcal{F}}\left(R_{1} \cdot\right) \\
\operatorname{ext}_{D}^{i}(M, \mathcal{F}) \cong \operatorname{ker}_{\mathcal{F}}\left(R_{i+1} \cdot\right) / \operatorname{im}_{\mathcal{F}}\left(R_{i} \cdot\right), \quad i \geq 1
\end{array}\right.
$$

Theorem 1 shows that:

$$
\operatorname{ext}_{D}^{0}(M, \mathcal{F})=\operatorname{hom}_{D}(M, \mathcal{F}) \text {. }
$$

See also, e.g., [50]. We say that the complex (6) is obtained by application of the contravariant left exact functor $\operatorname{hom}_{D}(\cdot, \mathcal{F})$ to the reduced (truncated) free resolution of $M$, namely, to the complex obtained by removing $M$ from the finite free resolution (3) as follows:

$$
\ldots \stackrel{. R_{4}}{\longrightarrow} D^{1 \times p_{3}} \stackrel{. R_{3}}{\longrightarrow} D^{1 \times p_{2}} \stackrel{. R_{2}}{\longrightarrow} D^{1 \times p_{1}} \stackrel{. R_{1}}{\longrightarrow} D^{1 \times p_{0}} \longrightarrow 0 .
$$

A fundamental theorem of homological algebra asserts that the abelian $\operatorname{groups}_{\operatorname{ext}}^{i}(M, \mathcal{F})$ 's depend only on the left $D$-modules $M$ and $\mathcal{F}$ (up to abelian group isomorphism), i.e., they do not depend on the choice of the free resolution (3) of $M$ (see, e.g., [50]). The $\operatorname{ext}_{D}^{i}(M, \mathcal{F})$ 's can also be defined using projective resolutions of $M$ (see, e.g., [50]). But, this approach is generally less constructive than the one based on free resolutions. In what follows, we shall only consider free resolutions and we let the reader reformulate the different results based on projective resolutions.

The idea of replacing a rather complicated left $D$-module $M$ by the complex (8) formed by the left $D$-modules $D^{1 \times p_{i}}$ 's (free modules) and trivial left $D$-homomorphisms.$R_{i}$ 's (defined by matrices) is of paramount importance in the theory of derived category developed by Grothendieck and Verdier (see, e.g., [25]). In this paper, we shall show how the grade filtration of $M$, which is difficult to compute directly on $M$, can be explicitly characterized by many but simple (matrix) computations related to the computation of $\operatorname{ext}_{D}^{i}(M, D)$ and $\operatorname{ext}_{D}^{j}\left(\operatorname{ext}_{D}^{i}(M, D), D\right)$.

Similarly, if $N$ a finitely generated right $D$-module and $\mathcal{G}$ a right $D$-module, then using a free resolution (4) of $N$, we can define the following abelian groups:

$$
\left\{\begin{array}{l}
\operatorname{ext}_{D}^{0}(N, \mathcal{G})=\operatorname{hom}_{D}(N, \mathcal{G}) \cong \operatorname{ker}_{\mathcal{G}}\left(. S_{1}\right), \\
\operatorname{ext}_{D}^{i}(N, \mathcal{G}) \cong \operatorname{ker}_{\mathcal{G}}\left(. S_{i+1}\right) / \operatorname{im}_{\mathcal{G}}\left(. S_{i}\right), \quad i \geq 1 .
\end{array}\right.
$$

We note that if $M$ is a left (resp., right) $D$-module, then $\operatorname{ext}_{D}^{i}(M, D)$ is a right (resp., left) $D$-module due to the $D-D$-bimodule structure of $D$ (see, e.g., [50]).

Definition $3([50])$. A left $D$-module $\mathcal{F}$ is injective if $\operatorname{ext}_{D}^{i}(M, \mathcal{F})=0$ for all left $D$-modules $M$ and for all $i \geq 1$.

Example 2. If $\Omega$ is an open convex subset of $\mathbb{R}^{n}$, then the space $C^{\infty}(\Omega)$ (resp., $\mathcal{D}^{\prime}(\Omega), \mathcal{S}^{\prime}(\Omega)$, $\mathcal{A}(\Omega), \mathcal{O}(\Omega)$ ) of smooth functions (resp., distributions/temperate distributions, real analytic/ holomorphic functions) on $\Omega$ is an injective $D=k\left[\partial_{1}, \ldots, \partial_{n}\right]$-module $(k=\mathbb{R}, \mathbb{C})[34,36$, 53. 
If $M$ is a finitely generated left $D$-module and $\mathcal{F}$ an injective left $D$-module, then applying the contravariant left exact functor $\operatorname{hom}_{D}(\cdot, \mathcal{F})$ to $(3)$, and using Theorem 1 and the fact that $\operatorname{ext}_{D}^{i}(\cdot, \mathcal{F})=0$ for all $i \geq 1$, we obtain the following exact sequence of abelian groups:

$$
\ldots \stackrel{R_{3} .}{\longleftarrow} \mathcal{F}^{p_{2}} \stackrel{R_{2} .}{\longleftarrow} \mathcal{F}^{p_{1}} \stackrel{R_{1}}{\longleftarrow} \mathcal{F}^{p_{0}} \longleftarrow \operatorname{hom}_{D}(M, \mathcal{F}) \longleftarrow 0 .
$$

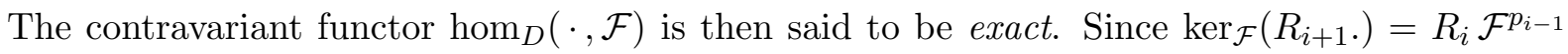
for all $i \geq 1$, the linear system $\operatorname{ker}_{\mathcal{F}}\left(R_{i+1}\right.$.) is then parametrized by $R_{i}$ (called a parametrization).

Let us now state two results which will be used in Section 3 ,

Theorem 3 ([50]). Let [2) be a short exact sequence of left (resp., right) D-modules and $N a$ left (resp., right) D-module. Then, the following long exact sequence holds

$$
\begin{aligned}
& 0 \longrightarrow \operatorname{ext}_{D}^{0}\left(M^{\prime \prime}, N\right) \stackrel{g^{\star}}{\longrightarrow} \operatorname{ext}_{D}^{0}(M, N) \stackrel{f^{\star}}{\longrightarrow} \operatorname{ext}_{D}^{0}\left(M^{\prime}, N\right) \\
& \longrightarrow \operatorname{ext}_{D}^{1}\left(M^{\prime \prime}, N\right) \longrightarrow \operatorname{ext}_{D}^{1}(M, N) \longrightarrow \operatorname{ext}_{D}^{1}\left(M^{\prime}, N\right) \\
& \longrightarrow \operatorname{ext}_{D}^{2}\left(M^{\prime \prime}, N\right) \longrightarrow \operatorname{ext}_{D}^{2}(M, N) \longrightarrow \ldots
\end{aligned}
$$

where $f^{\star}$ (resp., $\left.g^{\star}\right)$ is defined by $f^{\star}(\phi)=\phi \circ f\left(\right.$ resp., $\left.g^{\star}(\psi)=\psi \circ g\right)$ for all $\phi \in \operatorname{hom}_{D}(M, N)$ (resp., for all $\psi \in \operatorname{hom}_{D}\left(M^{\prime \prime}, N\right)$ ).

Remark 2. One can prove that a left $D$-module $M$ is projective iff $\operatorname{ext}_{D}^{i}(M, N)=0$ for all left $D$-module $N$ and for all $i \geq 1$ (see, e.g., [50]). If $P$ and $P^{\prime}$ are the two projective left $D$-modules considered in Remark 1, the additivity of the functor $\operatorname{ext}_{D}^{i}(\cdot, N)$ (see, e.g., [50]) then yields

$$
\forall i \geq 1, \quad\left\{\begin{array}{l}
\operatorname{ext}_{D}^{i}\left(\operatorname{ker} \pi \oplus P^{\prime}, N\right) \cong \operatorname{ext}_{D}^{i}(\operatorname{ker} \pi, N) \oplus \operatorname{ext}_{D}^{i}\left(P^{\prime}, N\right)=\operatorname{ext}_{D}^{i}(\operatorname{ker} \pi, N), \\
\operatorname{ext}_{D}^{i}\left(\operatorname{ker} \pi^{\prime} \oplus P, N\right) \cong \operatorname{ext}_{D}^{i}\left(\operatorname{ker} \pi^{\prime}, N\right) \oplus \operatorname{ext}_{D}^{i}(P, N)=\operatorname{ext}_{D}^{i}\left(\operatorname{ker} \pi^{\prime}, N\right),
\end{array}\right.
$$

and thus, $\operatorname{ext}_{D}^{i}(\operatorname{ker} \pi, N) \cong \operatorname{ext}_{D}^{i}\left(\operatorname{ker} \pi^{\prime}, N\right)$ for $i \geq 1$, which shows that $\operatorname{ext}_{D}^{i}(\operatorname{ker} \pi, N)$ depends only on $M$ and $N$ (up to isomorphism) for $i \geq 1$.

Combining Remark 2 with Theorem 3 , we obtain the following result.

Proposition 1 ([50]). Let [2) be a short exact sequence of left (resp., right) D-modules and $M$ a projective left (resp., right) $D$-module. Then, for every left (resp., right) $D$-module $N$, we have $\operatorname{ext}_{D}^{i+1}\left(M^{\prime \prime}, N\right) \cong \operatorname{ext}_{D}^{i}\left(M^{\prime}, N\right)$ for $i \geq 1$.

Let us introduce important invariants of modules and rings.

Definition 4 ([50]). $\quad 1$. The left projective dimension of a left $D$-module $M$, denoted by $\operatorname{lpd}_{D}(M)$, is the minimum of the lengths of projective resolutions of $M$. If no such integer exists, then we set $\operatorname{lpd}_{D}(M)=\infty$. Similarly for the right projective dimension $\operatorname{rpd}_{D}(N)$ of a right $D$-module $N$.

2. The left global dimension (resp., right global dimension) of a ring $D$, denoted by $\operatorname{lgd}(D)$ $($ resp., $\operatorname{rgd}(D))$, is the supremum of $\operatorname{lpd}_{D}(M)$ (resp., $\left.\operatorname{rpd}_{D}(N)\right)$ for all left $D$-modules $M$ (resp., all right $D$-modules $N$ ).

3. If the left and the right global dimension of $D$ coincide, then the common value is called the global dimension of $D$ and is denoted by $\operatorname{gld}(D)$. 
Proposition 2 ([10]). Let $D$ be a noetherian ring and $M$ a finitely generated left $D$-module. Then, we have:

$$
\operatorname{lpd}_{D}(M)=\sup \left\{i \in \mathbb{N} \mid \operatorname{ext}_{D}^{i}(M, D) \neq 0\right\} .
$$

Similarly for the right projective dimension $\operatorname{rpd}_{D}(N)$ of a right $D$-module $N$.

Proposition $3([50]) \cdot \operatorname{lgd}(D) \leq n$ iff $\operatorname{ext}_{D}^{i}(M, N)=0$ for all left $D$-modules $M$ and $N$, and for all $i>n$.

Theorem $4([\underline{50})$. If $D$ is a noetherian ring, then $\operatorname{lgld}(D)=\operatorname{rgld}(D)$.

Example 3. If $k$ is a field, then $\operatorname{gld}\left(k\left[x_{1}, \ldots, x_{n}\right]\right)=n$ [50. If $k$ is a field of characteristic 0, $k^{\prime}=\mathbb{R}$ or $\mathbb{C}$, and $D=A_{n}(k), B_{n}(k), \hat{\mathcal{D}}_{n}(k)$, or $\mathcal{D}_{n}\left(k^{\prime}\right)$, then $\operatorname{gld}(D)=n$ [9, 10, 30].

We are now in a position to recall how the properties stated in Definition 1 can be checked by means of homological techniques for a noetherian regular domain $D$, namely a noetherian domain $D$ of finite global dimension $\operatorname{gld}(D)$.

Theorem 5 (11, 14, 30, 38, 40]). Let $D$ be a noetherian domain with a finite global dimension $\operatorname{gld}(D)=n, M=D^{1 \times p} /\left(D^{1 \times q} R\right)$ a finitely presented left $D$-module, and $N=D^{q} /\left(R D^{p}\right)$ the so-called Auslander transpose right $D$-module of $M$.

1. The following left D-isomorphism holds:

$$
t(M) \cong \operatorname{ext}_{D}^{1}(N, D)
$$

2. $M$ is torsion-free iff $\operatorname{ext}_{D}^{1}(N, D)=0$.

3. The following long exact sequence holds

$$
0 \longrightarrow \operatorname{ext}_{D}^{1}(N, D) \longrightarrow M \stackrel{\varepsilon}{\longrightarrow} \operatorname{hom}_{D}\left(\operatorname{hom}_{D}(M, D), D\right) \longrightarrow \operatorname{ext}_{D}^{2}(N, D) \longrightarrow 0,
$$

where $\varepsilon$ is defined in 3 of Definition 1 .

4. $M$ is reflexive iff $\operatorname{ext}_{D}^{i}(N, D)=0$ for $i=1,2$.

5. $M$ is projective iff $\operatorname{ext}_{D}^{i}(N, D)=0$ for $i=1, \ldots, n$.

Remark 3. The Auslander transpose right $D$-module $N=D^{q} /\left(R D^{p}\right)$ depends on the left $D$-module $M=D^{1 \times p} /\left(D^{1 \times q} R\right)$ up to a projective equivalence: if $M \cong M^{\prime}=D^{1 \times p^{\prime}} /\left(D^{1 \times q^{\prime}} R^{\prime}\right)$, then $N \oplus D^{\left(p+q^{\prime}\right)} \cong N^{\prime} \oplus D^{\left(p^{\prime}+q\right)}$, where $N^{\prime}=D^{q^{\prime}} /\left(R^{\prime} D^{p^{\prime}}\right)$ [1. See [18] for a constructive proof. Using Remark 2, the additivity of the functor $\operatorname{ext}_{D}^{i}(\cdot, \mathcal{F})$ (see, e.g., [50]) then yields $\operatorname{ext}_{D}^{i}(N, \mathcal{F}) \cong \operatorname{ext}_{D}^{i}\left(N^{\prime}, \mathcal{F}\right)$ for all left $D$-modules $\mathcal{F}$ and for $i \geq 1$. Therefore, the results stated in Theorem 5 do not depend on the chosen presentation of $M$.

Theorem 5 was implemented in the OREMODULES package [15] for the class of Ore algebras of functional operators implemented in the Maple package Ore_algebra (e.g., PD, shift, difference, time-delay operators) for which Buchberger's algorithm terminates for any admissible term order and which computes a Gröbner basis [14]. Using the OrEMODUlEs package, we can effectively check whether or not the left $D$-module $M=D^{1 \times p} /\left(D^{1 \times q} R\right)$ admits torsion elements or is torsion-free, reflexive or projective. For applications of Theorem 5 to mathematical systems theory and mathematical physics, see [15]. 
Let us recall how to compute the torsion left $D$-submodule $t(M)$ of $M=D^{1 \times p} /\left(D^{1 \times q} R\right)$. We first consider $Q \in D^{p \times m}$ such that $\operatorname{ker}_{D}(R$. $)=Q D^{m}$. Then, we get the exact sequence $0 \longleftarrow N \longleftarrow D^{q} \stackrel{R .}{\longleftarrow} D^{p} \stackrel{Q .}{\longleftarrow} D^{m}$. Then, 1 of Theorem 5 shows that the defect of exactness at $D^{1 \times p}$ of the complex $D^{1 \times q} \stackrel{. R}{\longrightarrow} D^{1 \times p} \stackrel{\cdot Q}{\longrightarrow} D^{1 \times m}$ is defined by

$$
\operatorname{ext}_{D}^{1}(N, D) \cong t(M)=\operatorname{ker}_{D}(. Q) / \operatorname{im}_{D}(. R)=\left(D^{1 \times q^{\prime}} R^{\prime}\right) /\left(D^{1 \times q} R\right),
$$

where $R^{\prime} \in D^{q^{\prime} \times p}$ is any matrix such that $\operatorname{ker}_{D}(. Q)=D^{1 \times q^{\prime}} R^{\prime}$. Moreover, the standard third isomorphism theorem [50] then yields:

$$
M / t(M)=\left[D^{1 \times p} /\left(D^{1 \times q} R\right)\right] /\left[\left(D^{1 \times q^{\prime}} R^{\prime}\right) /\left(D^{1 \times q} R\right)\right] \cong D^{1 \times p} /\left(D^{1 \times q^{\prime}} R^{\prime}\right) .
$$

We note that a right analogous of Theorem 1 asserts that $\operatorname{hom}_{D}(M, D) \cong \operatorname{ker}_{D}(R$.). Hence, if $\operatorname{hom}_{D}(M, D)=0$, then $0 \longleftarrow N \longleftarrow D^{q} \stackrel{R}{\longleftarrow} D^{p} \longleftarrow 0$ is an exact sequence, and thus the defect of exactness of the complex $D^{1 \times q} \stackrel{. R}{\longrightarrow} D^{1 \times p} \longrightarrow 0$ at $D^{1 \times p}$ is $\operatorname{ext}_{D}^{1}(N, D) \cong t(M)=$ $D^{1 \times p} /\left(D^{1 \times q} R\right)=M$ by $(9)$, i.e., $M$ is a torsion left $D$-module. Conversely, if $M$ is a torsion left $D$-module and $f \in \operatorname{hom}_{D}(M, D)$, then for every $m \in M$, there exists $d \in D \backslash\{0\}$ such that $d m=0$, which yields $d f(m)=f(d m)=0$, and thus $f(m)=0$ since $D$ is a domain and $f(m) \in D$. Thus, $f=0$, i.e., $\operatorname{hom}_{D}(M, D)=0$. We obtain the following corollary of Theorem 5 .

Corollary 1 (see, e.g., [14). Let $M$ be a finitely generated left module over a noetherian domain $D$. Then, $M$ is a torsion left $D$-module iff $\operatorname{hom}_{D}(M, D)=0$.

Let us now introduce a lemma which gives a finite presentation of a factor module.

Proposition 4 (see, e.g. [16]). Let $R \in D^{q \times p}$ and $R^{\prime} \in D^{q^{\prime} \times p}$ satisfy $D^{1 \times q} R \subseteq D^{1 \times q^{\prime}} R^{\prime}$, i.e., are such that $R=R^{\prime \prime} R^{\prime}$ for a certain $R^{\prime \prime} \in D^{q \times q^{\prime}}$. Moreover, let $R_{2}^{\prime} \in D^{r^{\prime} \times q^{\prime}}$ be a matrix such that $\operatorname{ker}_{D}\left(. R^{\prime}\right)=D^{1 \times r^{\prime}} R_{2}^{\prime}$, and let $\pi$ and $\pi^{\prime}$ be respectively the following canonical projections:

$$
\pi: D^{1 \times q^{\prime}} R^{\prime} \longrightarrow\left(D^{1 \times q^{\prime}} R^{\prime}\right) /\left(D^{1 \times q} R\right), \quad \pi^{\prime}: D^{1 \times q^{\prime}} \longrightarrow D^{1 \times q^{\prime}} /\left(D^{1 \times q} R^{\prime \prime}+D^{1 \times r^{\prime}} R_{2}^{\prime}\right) .
$$

Then, the left D-homomorphism ı defined by

$$
\begin{aligned}
D^{1 \times q^{\prime}} /\left(D^{1 \times q} R^{\prime \prime}+D^{1 \times r^{\prime}} R_{2}^{\prime}\right) & \stackrel{\iota}{ }\left(D^{1 \times q^{\prime}} R^{\prime}\right) /\left(D^{1 \times q} R\right) \\
\pi^{\prime}(\lambda) & \longmapsto \pi\left(\lambda R^{\prime}\right),
\end{aligned}
$$

is an isomorphism and its inverse $\iota^{-1}$ is defined by:

$$
\begin{aligned}
\left(D^{1 \times q^{\prime}} R^{\prime}\right) /\left(D^{1 \times q} R\right) & \stackrel{\iota^{-1}}{\longrightarrow} D^{1 \times q^{\prime}} /\left(D^{1 \times q} R^{\prime \prime}+D^{1 \times r^{\prime}} R_{2}^{\prime}\right) \\
\pi\left(\lambda R^{\prime}\right) & \longmapsto \pi^{\prime}(\lambda) .
\end{aligned}
$$

Applying Proposition 4 to the left $D$-module $t(M)=\left(D^{1 \times q^{\prime}} R^{\prime}\right) /\left(D^{1 \times q} R\right)$, we obtain

$$
t(M) \cong D^{1 \times q^{\prime}} /\left(D^{1 \times q} R^{\prime \prime}+D^{1 \times r^{\prime}} R_{2}^{\prime}\right)=D^{1 \times q^{\prime}} /\left(D^{1 \times\left(q+r^{\prime}\right)}\left(R^{\prime \prime T} \quad R_{2}^{\prime T}\right)^{T}\right),
$$

where $R^{\prime \prime} \in D^{q \times q^{\prime}}$ and $R_{2}^{\prime} \in D^{r^{\prime} \times q^{\prime}}$ are defined by $R=R^{\prime \prime} R^{\prime}$ and $\operatorname{ker}_{D}\left(. R^{\prime}\right)=D^{1 \times r^{\prime}} R_{2}^{\prime}$.

If $t(M)=0$, then using 111 , the complex $D^{1 \times q} \stackrel{. R}{\longrightarrow} D^{1 \times p} \stackrel{. Q}{\longrightarrow} D^{1 \times m}$ is exact at $D^{1 \times p}$, and thus it defines the beginning of a free resolution of the left $D$-module $L=D^{1 \times m} /\left(D^{1 \times q} Q\right)$. Up to isomorphism, a finitely generated torsion-free left $D$-module $M$ can then be embedded into a finite free left $D$-module since $M=D^{1 \times p} /\left(D^{1 \times q} R\right) \cong \operatorname{im}_{D}(. Q) \subseteq D^{1 \times m}$. If $\mathcal{F}$ is an injective 
left $D$-module, then applying the exact functor $\operatorname{hom}_{D}(\cdot, \mathcal{F})$ to the above beginning of a free resolution of $L$, we obtain the exact sequence $\mathcal{F}^{q} \stackrel{R \text {. }}{\longleftarrow} \mathcal{F}^{p} \stackrel{Q .}{\longleftarrow} \mathcal{F}^{m}$, i.e., $\operatorname{ker}_{\mathcal{F}}(R)=.Q \mathcal{F}^{m}$, i.e., $Q$ is a parametrization of $\operatorname{ker}_{\mathcal{F}}(R$.). The computation of parametrizations is implemented in the OreModules package. This package allows one to explicitly parametrize underdetermined linear functional systems appearing in mathematical physics and in control theory (see [15]).

The above techniques will be generalized in Section 3 to determine the so-called grade filtration of a finitely generated left $D$-module $M$.

To finish with this section, we shortly recall a few classical results on homomorphisms of finitely presented modules that will be used in the next sections.

Proposition 5 ([16, 18]). Let $M=D^{1 \times p} /\left(D^{1 \times q} R\right)$ (resp., $\left.M^{\prime}=D^{1 \times p^{\prime}} /\left(D^{1 \times q^{\prime}} R^{\prime}\right)\right)$ be a left $D$-module finitely presented by $R \in D^{q \times p}$ (resp., $R^{\prime} \in D^{q^{\prime} \times p^{\prime}}$ ), and $\pi: D^{1 \times p} \longrightarrow M$ (resp., $\left.\pi^{\prime}: D^{1 \times p^{\prime}} \longrightarrow M^{\prime}\right)$ the canonical projection onto $M$ (resp., $\left.M^{\prime}\right)$. Then, every $f \in \operatorname{hom}_{D}\left(M, M^{\prime}\right)$ is defined by $f(\pi(\lambda))=\pi^{\prime}(\lambda P)$ for all $\lambda \in D^{1 \times p}$, where $P \in D^{p \times p^{\prime}}$ satisfies $R P=Q R^{\prime}$ for a certain $Q \in D^{q \times q^{\prime}}$. Moreover, we have:

1. $\operatorname{ker} f=\left(D^{1 \times r} S\right) /\left(D^{1 \times q} R\right)$, where the matrix $S \in D^{r \times p}$ is defined by:

$$
\operatorname{ker}_{D}\left(.\left(P^{T} \quad R^{\prime T}\right)^{T}\right)=D^{1 \times r}(S \quad-T), \quad T \in D^{r \times q^{\prime}} .
$$

In particular, $f$ is injective iff there exists a matrix $F \in D^{r \times q}$ such that $S=F R$.

2. $\operatorname{im} f=\left(D^{1 \times p} P+D^{1 \times q^{\prime}} R^{\prime}\right) /\left(D^{1 \times q^{\prime}} R^{\prime}\right) \cong \operatorname{coim} f=D^{1 \times p} /\left(D^{1 \times r} S\right)$.

3. coker $f=D^{1 \times p^{\prime}} /\left(D^{1 \times p} P+D^{1 \times q^{\prime}} R^{\prime}\right)$. Thus, $f$ is surjective iff $\left(P^{T} \quad R^{\prime T}\right)^{T}$ admits a left inverse over $D$, i.e., $X \in D^{p^{\prime} \times p}$ and $Y \in D^{p^{\prime} \times q^{\prime}}$ exist such that $X P+Y R^{\prime}=I_{p^{\prime}}$.

4. $f$ is an isomorphism, i.e., $M \cong M^{\prime}$, iff there exists $F \in D^{r \times q}$ such that $S=F R$ and the matrix $\left(\begin{array}{ll}P^{T} & R^{T}\end{array}\right)^{T}$ admits a left inverse over $D$. If $X \in D^{p^{\prime} \times p}$ is defined as in 3, then $f^{-1} \in \operatorname{hom}_{D}\left(M^{\prime}, M\right)$ is defined by $f^{-1}\left(\pi^{\prime}\left(\lambda^{\prime}\right)\right)=\pi\left(\lambda^{\prime} X\right)$ for all $\lambda^{\prime} \in D^{1 \times p^{\prime}}$.

\subsection{Baer's extensions}

In this section, we give another interpretation of the abelian group $\operatorname{ext}_{D}^{1}(M, N)$ which will be used in Section 4. To do that, let us introduce a few more definitions (see, e.g., [50]).

Definition 5. 1. Let $M$ and $N$ be two left $D$-modules. An extension of $M$ by $N$ is a short exact sequence of left $D$-modules of the form:

$$
e: 0 \longrightarrow N \stackrel{\alpha}{\longrightarrow} E \stackrel{\beta}{\longrightarrow} M \longrightarrow 0 .
$$

2. Two extensions $e_{i}: 0 \longrightarrow N \stackrel{\alpha_{i}}{\longrightarrow} E_{i} \stackrel{\beta_{i}}{\longrightarrow} M \longrightarrow 0$ of $M$ by $N$ for $i=1,2$ are said to be equivalent, which is denoted by $e_{1} \sim e_{2}$, if there exists a left $D$-isomorphism $\phi: E_{1} \longrightarrow E_{2}$ such that $\alpha_{2}=\phi \circ \alpha_{1}$ and $\beta_{1}=\beta_{2} \circ \phi$, or equivalently, such that the following commutative exact diagram holds:

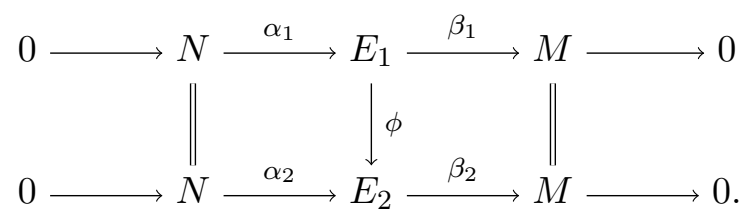


3. Let $[e]$ be the equivalence class of the extension $e$ for the equivalence relation $\sim$. The set of all equivalence classes of extensions of $M$ by $N$ is denoted by $\mathrm{e}_{D}(M, N)$.

The next theorem, which can be traced back to Baer's work, plays an important role in homological algebra. In particular, it explains the terminology extension used for $\operatorname{ext}_{D}^{1}(M, N)$.

Theorem 6 ([50]). Let $M$ and $N$ be two left D-modules. Then, we have:

$$
\operatorname{ext}_{D}^{1}(M, N) \cong \mathrm{e}_{D}(M, N) .
$$

The next theorem gives an explicit description of the isomorphism stated in Theorem 6 in the case where $M$ and $N$ are two finitely presented left $D$-modules.

Theorem 7 ([46, 47]). Let $M=D^{1 \times p} /\left(D^{1 \times q} R\right)$ and $N=D^{1 \times s} /\left(D^{1 \times t} S\right), \pi: D^{1 \times p} \longrightarrow M$ (resp., $\delta: D^{1 \times s} \longrightarrow N$ ) be the canonical projection onto $M$ (resp., $N$ ), and $R_{2} \in D^{r \times q}$ a matrix such that $\operatorname{ker}_{D}(. R)=D^{1 \times r} R_{2}$, and $\Omega=\left\{X \in D^{q \times s} \mid \exists Y \in D^{r \times t}: R_{2} X=Y S\right\}$. Then, every equivalence class of extensions of $M$ by $N$ is defined by the following short exact sequence

$$
e: 0 \longrightarrow N \stackrel{\alpha}{\longrightarrow} E \stackrel{\beta}{\longrightarrow} M \longrightarrow 0,
$$

where $E=D^{1 \times(p+s)} /\left(D^{1 \times(q+t)} L\right)$ and $L=\left(\begin{array}{cc}R & -A \\ 0 & S\end{array}\right) \in D^{(q+t) \times(p+s)}$ for a certain $A \in \Omega$,

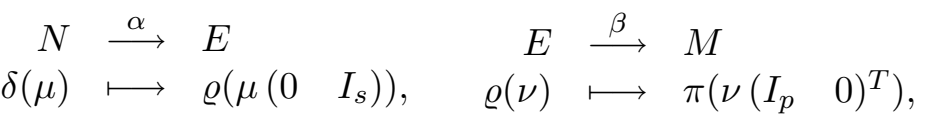

and $\varrho: D^{1 \times(p+s)} \longrightarrow E$ is the canonical projection onto E. Finally, the equivalence class $[e]$ depends only on the residue class $\epsilon(A)$ of the matrix $A$ in the following abelian group:

$$
\Omega /\left(R D^{p \times s}+D^{q \times t} S\right) \cong \operatorname{ext}_{D}^{1}(M, N) .
$$

Remark 4. The extension $e$ of Theorem 7 is trivial, i.e., $E \cong N \oplus M$, iff there exist $U \in D^{p \times s}$ and $V \in D^{q \times t}$ such that $A=R U+V S$, i.e., iff $\epsilon(A)=0$. If $D$ is a commutative polynomial ring over a computable field $k$, then using Kronecker product and Gröbner/Janet bases, we can check whether or not this identity holds and if so, compute solutions $U$ and $V$. See, e.g., [47, 54,

The next corollary shows how to determine $\epsilon(A)$ for a given extension $e$ of $M$ by $N$.

Corollary 2 (47]). With the notations of Theorem 7, let $e^{\prime}: 0 \longrightarrow N \stackrel{u}{\longrightarrow} F \stackrel{v}{\longrightarrow} M \longrightarrow 0$ be an extension of the left $D$-module $M=D^{1 \times p} /\left(D^{1 \times q} R\right)$ by the left $D$-module $N=D^{1 \times s} /\left(D^{1 \times t} S\right)$, $\left\{f_{j}\right\}_{j=1, \ldots, p}$ (resp., $\left\{e_{i}\right\}_{i=1, \ldots, q}$ ) the standard basis of $D^{1 \times p}$ (resp., $\left.D^{1 \times q}\right), y_{j}=\pi\left(f_{j}\right)$, and $z_{j} \in F$ a pre-image of $y_{j}$ under $v$ for all $j=1, \ldots, p$. Then, we have $\sum_{j=1}^{p} R_{i j} z_{j} \in \operatorname{im} u$ for all $i=1, \ldots, q$, and, since $u$ is injective, there exists a unique $n_{i} \in N$ satisfying $u\left(n_{i}\right)=\sum_{j=1}^{p} R_{i j} z_{j}$. If we consider a pre-image $a_{i} \in D^{1 \times s}$ of $n_{i}$ under $\delta$, i.e., $n_{i}=\delta\left(a_{i}\right)$ for all $i=1, \ldots, q$, then the extensions $e^{\prime}$ and (16) are equivalent, where $E=D^{1 \times(p+s)} /\left(D^{1 \times(q+t)} L\right)$ and:

$$
L=\left(\begin{array}{cc}
R & -A \\
0 & S
\end{array}\right) \in D^{(q+t) \times(p+s)}, \quad A=\left(\begin{array}{c}
a_{1} \\
\vdots \\
a_{q}
\end{array}\right) \in D^{q \times s} .
$$


Equivalently, the following commutative exact diagram holds

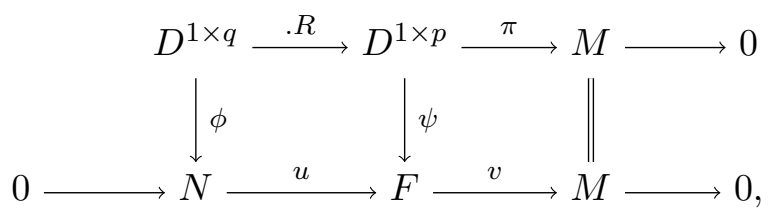

where $\psi$ and $\phi$ are respectively defined by:

$$
\begin{aligned}
& \psi: D^{1 \times p} \longrightarrow F \quad \phi: D^{1 \times q} \longrightarrow N \\
& f_{j} \longmapsto z_{j}, j=1, \ldots, p, \quad e_{i} \longmapsto n_{i}=\delta\left(a_{i}\right), i=1, \ldots, q .
\end{aligned}
$$

Theorem 7 and Corollary 2 will be abundantly used in Section 4. For more results on Baer's extensions, examples and applications to mathematical systems theory, see [4, 46, 47, 50, 54,

The next proposition shows how the presentation of the left $D$-module $E$ defining the extension of $M$ by $N$ (see Theorem 7 ) changes with the presentations of $M$ and $N$.

Proposition 6. With the notations of Theorem 7 , let $M=D^{1 \times p} /\left(D^{1 \times q} R\right), N=D^{1 \times s} /\left(D^{1 \times t} S\right)$, and $E=D^{1 \times(p+s)} /\left(D^{1 \times(q+t)} L\right)$ be three left $D$-modules defining the extension e of $M$ by $N(16)$. Moreover, let $f$ and $g$ be two left D-isomorphisms defined by

$$
\begin{aligned}
f: M=D^{1 \times p} /\left(D^{1 \times q} R\right) & \longrightarrow M^{\prime}=D^{1 \times p^{\prime}} /\left(D^{1 \times q^{\prime}} R^{\prime}\right) \\
\pi(\lambda) & \longmapsto \pi^{\prime}(\lambda P), \\
g: N=D^{1 \times s} /\left(D^{1 \times t} S\right) & \longrightarrow N^{\prime}=D^{1 \times s^{\prime}} /\left(D^{1 \times t^{\prime}} S^{\prime}\right) \\
\delta(\mu) & \longmapsto \delta^{\prime}(\mu X),
\end{aligned}
$$

where $\pi^{\prime}$ (resp., $\delta^{\prime}$ ) is the canonical projection onto $M^{\prime}$ (resp., $N^{\prime}$ ), i.e., $P \in D^{p \times p^{\prime}}, X \in D^{s \times s^{\prime}}$ are such that there exist $Q \in D^{q \times q^{\prime}}, P^{\prime} \in D^{p^{\prime} \times p}, Q^{\prime} \in D^{q^{\prime} \times q}, Y \in D^{t \times t^{\prime}}, X^{\prime} \in D^{s^{\prime} \times s}, Y^{\prime} \in D^{t^{\prime} \times t}$, $T \in D^{p \times q}, T^{\prime} \in D^{p^{\prime} \times q^{\prime}}, Z \in D^{s \times t}$, and $Z^{\prime} \in D^{s^{\prime} \times t^{\prime}}$ satisfying the following identities:

$$
\left\{\begin{array} { l } 
{ R P = Q R ^ { \prime } , } \\
{ R ^ { \prime } P ^ { \prime } = Q ^ { \prime } R , } \\
{ I _ { p } = P P ^ { \prime } + T R , } \\
{ I _ { p ^ { \prime } } = P ^ { \prime } P + T ^ { \prime } R ^ { \prime } , }
\end{array} \quad \left\{\begin{array}{l}
S X=Y S^{\prime}, \\
S^{\prime} X^{\prime}=Y^{\prime} S, \\
I_{s}=X X^{\prime}+Z S, \\
I_{s^{\prime}}=X^{\prime} X+Z^{\prime} S^{\prime} .
\end{array}\right.\right.
$$

Then, the extension e yields the following extension of $M^{\prime}$ by $N^{\prime}$

$$
e^{\prime}: 0 \longrightarrow N^{\prime} \stackrel{\alpha \circ g^{-1}}{\longrightarrow} E \stackrel{f \circ \beta}{\longrightarrow} M^{\prime} \longrightarrow 0,
$$

which implies that the left D-module $E$ admits the following presentation

$$
L^{\prime}=\left(\begin{array}{cc}
R^{\prime} & -Q^{\prime} A X \\
0 & S^{\prime}
\end{array}\right) \in D^{\left(q^{\prime}+t^{\prime}\right) \times\left(p^{\prime}+s^{\prime}\right)},
$$

i.e., $E \cong E^{\prime}=D^{1 \times\left(p^{\prime}+s^{\prime}\right)} /\left(D^{1 \times\left(q^{\prime}+t^{\prime}\right)} L^{\prime}\right)$, where this left D-isomorphism is explicitly defined by

$$
\begin{aligned}
& \varphi: E \longrightarrow E^{\prime} \quad \varphi^{-1}: E^{\prime} \longrightarrow E \\
& \varrho(\nu) \longmapsto \varrho^{\prime}(\nu U), \quad \varrho^{\prime}\left(\nu^{\prime}\right) \longmapsto \varrho\left(\nu^{\prime} U^{\prime}\right), \\
& U=\left(\begin{array}{cc}
P & T A X \\
0 & X
\end{array}\right) \in D^{(p+s) \times\left(p^{\prime}+s^{\prime}\right)}, \quad U^{\prime}=\left(\begin{array}{cc}
P^{\prime} & 0 \\
0 & X^{\prime}
\end{array}\right) \in D^{\left(p^{\prime}+s^{\prime}\right) \times(p+s)},
\end{aligned}
$$

and $\varrho^{\prime}: D^{1 \times\left(p^{\prime}+s^{\prime}\right)} \longrightarrow E^{\prime}$ is the canonical projection onto $E^{\prime}$. 
Proof. With the notations (18), 4 of Proposition 5 yields:

$$
\begin{aligned}
f^{-1}: M^{\prime}=D^{1 \times p^{\prime}} /\left(D^{1 \times q^{\prime}} R^{\prime}\right) & \longrightarrow M=D^{1 \times p} /\left(D^{1 \times q} R\right) \\
\pi^{\prime}\left(\lambda^{\prime}\right) & \longmapsto \pi\left(\lambda^{\prime} P^{\prime}\right), \\
g^{-1}: N^{\prime}=D^{1 \times s^{\prime}} /\left(D^{1 \times t^{\prime}} S^{\prime}\right) & \longrightarrow N=D^{1 \times s} /\left(D^{1 \times t} S\right) \\
\delta^{\prime}\left(\mu^{\prime}\right) & \longmapsto \delta\left(\mu^{\prime} X^{\prime}\right) .
\end{aligned}
$$

Using (18), we get $\left(I_{q}-Q Q^{\prime}-R T\right) R=R-Q Q^{\prime} R-R T R=R-R P P^{\prime}-R T R=0$. Thus, if $\operatorname{ker}_{D}(. R)=D^{1 \times r} R_{2}$, then there exists $T_{2} \in D^{q \times r}$ such that:

$$
I_{q}=Q Q^{\prime}+R T+T_{2} R_{2}
$$

Now, clearly, 16) yields (19). Moreover, since $A \in \Omega$ (see Theorem 7), there exists $B \in D^{r \times s}$ such that $R_{2} A=B S$. Hence, using this identity, (18) and (20), we obtain

$$
\begin{aligned}
L U & =\left(\begin{array}{cc}
R & -A \\
0 & S
\end{array}\right)\left(\begin{array}{cc}
P & T A X \\
0 & X
\end{array}\right)=\left(\begin{array}{cc}
R P & \left(R T-I_{q}\right) A X \\
0 & S X
\end{array}\right) \\
& =\left(\begin{array}{cc}
Q R^{\prime} & -\left(Q Q^{\prime} A+T_{2}\left(R_{2} A\right)\right) X \\
0 & Y S^{\prime}
\end{array}\right)=\left(\begin{array}{cc}
Q R^{\prime} & -\left(Q Q^{\prime} A+T_{2} B S\right) X \\
0 & Y S^{\prime}
\end{array}\right) \\
& =\left(\begin{array}{cc}
Q R^{\prime} & -Q Q^{\prime} A X-T_{2} B Y S^{\prime} \\
0 & Y S^{\prime}
\end{array}\right)=\left(\begin{array}{cc}
Q & -T_{2} B Y \\
0 & Y
\end{array}\right)\left(\begin{array}{cc}
R^{\prime} & -Q^{\prime} A X \\
0 & S^{\prime}
\end{array}\right)=V L^{\prime},
\end{aligned}
$$

where $V$ is the first matrix appearing in the last but one equality, which shows that $\varphi$ is welldefined by Proposition 5. Similarly, using (18), we get

$$
\begin{aligned}
L^{\prime} U^{\prime} & =\left(\begin{array}{cc}
R^{\prime} & -Q^{\prime} A X \\
0 & S^{\prime}
\end{array}\right)\left(\begin{array}{cc}
P^{\prime} & 0 \\
0 & X^{\prime}
\end{array}\right)=\left(\begin{array}{cc}
R^{\prime} P^{\prime} & -Q^{\prime} A X X^{\prime} \\
0 & S^{\prime} X^{\prime}
\end{array}\right) \\
& =\left(\begin{array}{cc}
Q^{\prime} R & -Q^{\prime} A\left(I_{s}-Z S\right) \\
0 & Y^{\prime} S
\end{array}\right)=\left(\begin{array}{cc}
Q^{\prime} & Q^{\prime} A Z \\
0 & Y^{\prime}
\end{array}\right)\left(\begin{array}{cc}
R & -A \\
0 & S
\end{array}\right)=V^{\prime} L,
\end{aligned}
$$

where $V^{\prime}$ is the first matrix appearing in the last but one equality, which yields $\phi \in \operatorname{hom}_{D}\left(E^{\prime}, E\right)$ defined by $\phi\left(\varrho^{\prime}\left(\nu^{\prime}\right)\right)=\varrho\left(\nu^{\prime} U^{\prime}\right)$ for all $\nu^{\prime} \in D^{1 \times\left(p^{\prime}+s^{\prime}\right)}$ by Proposition 5 . Using (18), we also have

$$
\begin{aligned}
U U^{\prime} & =\left(\begin{array}{cc}
P & T A X \\
0 & X
\end{array}\right)\left(\begin{array}{cc}
P^{\prime} & 0 \\
0 & X^{\prime}
\end{array}\right)=\left(\begin{array}{cc}
P P^{\prime} & T A X X^{\prime} \\
0 & X X^{\prime}
\end{array}\right) \\
& =\left(\begin{array}{cc}
I_{p}-T R & T A\left(I_{s}-Z S\right) \\
0 & I_{s}-Z S
\end{array}\right)=I_{p+s}-\left(\begin{array}{cc}
T & -T A Z \\
0 & Z
\end{array}\right)\left(\begin{array}{cc}
R & -A \\
0 & S
\end{array}\right),
\end{aligned}
$$

which shows that $\phi \circ \varphi=\operatorname{id}_{E}$. Moreover, using (18), we obtain

$$
\left(P^{\prime} T-T^{\prime} Q^{\prime}\right) R=P^{\prime} T R-T^{\prime} Q^{\prime} R=P^{\prime} T R-T^{\prime} R^{\prime} P^{\prime}=P^{\prime}\left(I_{p}-P P^{\prime}\right)-\left(I_{p^{\prime}}-P^{\prime} P\right) P^{\prime}=0,
$$

which shows that there exists $L \in D^{p^{\prime} \times r}$ such that $P^{\prime} T-T^{\prime} Q^{\prime}=L R_{2}$. Using $R_{2} A=B S$ and $S X=Y S^{\prime}$ (see (18)), $\left(P^{\prime} T-T^{\prime} Q^{\prime}\right) A X=L\left(R_{2} A\right) X=L B S X=L B Y S^{\prime}$, and then

$$
\begin{aligned}
U U^{\prime} & =\left(\begin{array}{cc}
P^{\prime} & 0 \\
0 & X^{\prime}
\end{array}\right)\left(\begin{array}{cc}
P & T A X \\
0 & X
\end{array}\right)=\left(\begin{array}{cc}
P^{\prime} P & P^{\prime} T A X \\
0 & X^{\prime} X
\end{array}\right) \\
& =\left(\begin{array}{cc}
I_{p^{\prime}}-T^{\prime} R^{\prime} & P^{\prime} T A X \\
0 & I_{s^{\prime}}-Z^{\prime} S^{\prime}
\end{array}\right)=I_{p^{\prime}+s^{\prime}}-\left(\begin{array}{cc}
T^{\prime} & -L B Y \\
0 & Z^{\prime}
\end{array}\right)\left(\begin{array}{cc}
R^{\prime} & -Q^{\prime} A X \\
0 & S^{\prime}
\end{array}\right),
\end{aligned}
$$


which shows that $\varphi \circ \phi=\mathrm{id}_{E^{\prime}}$, and thus proves that $\varphi$ is a left $D$-isomorphism and $\phi=\varphi^{-1}$.

\subsection{Pure modules and grade filtration}

Let us introduce the concept of the grade number of a finitely generated left $D$-module $M$.

Definition $6([9,10])$. The grade number of a nonzero finitely generated left $D$-module $M$ is defined by $j_{D}(M)=\inf \left\{i \in \mathbb{N} \mid \operatorname{ext}_{D}^{i}(M, D) \neq 0\right\}$. If $M=0$, then we set $j_{D}(M)=\infty$. A similar definition holds for right $D$-modules.

If $M \neq 0$, then $j_{D}(M)$ is then the smallest integer such that $\operatorname{ext}_{D}^{j_{D}(M)}(M, D) \neq 0$.

Remark 5. If $\operatorname{gld}(D)$ is finite and $M$ is a nonzero left $D$-module, then using Proposition 3 , $\operatorname{ext}_{D}^{i}(M, D)=0$ for all $i>\operatorname{gld}(D)$, which yield $0 \leq j_{D}(M) \leq \operatorname{gld}(D)$.

Let us now introduce the concept of pure module that will play an important role.

Definition $7([10])$. A finitely generated left $D$-module $M$ is said to be pure or $j_{D}(M)$-pure if $j_{D}(N)=j_{D}(M)$ for all nonzero left $D$-submodules $N$ of $M$.

Remark 6. If $M$ is a pure left $D$-module, then for every $m \in M \backslash\{0\}$, the cyclic left $D$-module $D m$ generated by $m$ satisfies $j_{D}(D m)=j_{D}(M)$. More generally, if $N$ is a left $D$-submodule of a $j_{D}(M)$-pure left $D$-module $M$, then $N$ is also a $j_{D}(M)$-pure left $D$-module since every left $D$-submodule of $N$ is a left $D$-submodule of $M$ and $j_{D}(N)=j_{D}(M)$.

In what follows, we shall mainly focus on the class of Auslander regular rings.

Definition 8 ([10]). A ring $D$ is called an Auslander regular ring if $D$ is a noetherian ring of finite global dimension $\operatorname{gld}(D)$ which satisfies the Auslander condition, namely, for every $i \in \mathbb{N}$, for every finitely generated left (resp., right) $D$-module $M$, and for every left (resp., right) $D$-submodule $N$ of $\operatorname{ext}_{D}^{i}(M, D)$, then $j_{D}(N) \geq i$.

Remark 7. If $D$ is an Auslander regular ring, then for a nonzero finitely generated left $D$-module $M$, taking $N=\operatorname{ext}_{D}^{i}(M, D)$ in Definition 8, we get $j_{D}\left(\operatorname{ext}_{D}^{i}(M, D)\right) \geq i$, i.e., $\operatorname{ext}_{D}^{j}\left(\operatorname{ext}_{D}^{i}(M, D), D\right)=0$ for $0 \leq j<i$. Similarly, considering $\operatorname{ext}_{D}^{i}(M, D)$ instead of $M$ in Definition 8, then $N \subseteq \operatorname{ext}_{D}^{i}\left(\operatorname{ext}_{D}^{i}(M, D), D\right) \neq 0$ yields $j_{D}(N) \geq i$.

Theorem 8 ([10]). Let $D$ be an Auslander regular ring and $M$ a nonzero finitely generated left $D$-module. Then, we have:

1. $M$ is pure iff $M$ is a left $D$-submodule of $\operatorname{ext}_{D}^{j_{D}(M)}\left(\operatorname{ext}_{D}^{j_{D}(M)}(M, D), D\right)$.

2. $M$ is pure iff $\operatorname{ext}_{D}^{i}\left(\operatorname{ext}_{D}^{i}(M, D), D\right)=0$ for $i \neq j_{D}(M)$.

3. If $\operatorname{ext}_{D}^{i}\left(\operatorname{ext}_{D}^{i}(M, D), D\right) \neq 0$, then $\operatorname{ext}_{D}^{i}\left(\operatorname{ext}_{D}^{i}(M, D), D\right)$ is a pure left D-module with grade number $i$, i.e., $j_{D}\left(\operatorname{ext}_{D}^{i}\left(\operatorname{ext}_{D}^{i}(M, D), D\right)\right)=i$.

Example 4. By 1 of Theorem 8, $M$ is 0-pure iff $M$ is a left $D$-submodule of $\operatorname{hom}_{D}\left(\operatorname{hom}_{D}(M, D), D\right)$. If $D$ is a domain, then using 3 of Theorem 5 , we deduce that $M$ is 0 -pure iff $M$ is a torsion-free left $D$-module. In particular, the left $D$-module $M / t(M)$ is either zero or 0-pure. 
Let us now show that pure modules naturally appear in the study of a finitely generated left module $M$ over an Auslander regular ring $D$. Let us consider:

$$
t_{i}(M)=\left\{m \in M \mid j_{D}(D m) \geq i\right\}, \quad i=0, \ldots, n=\operatorname{gld}(D), \quad t_{n+1}(M)=0 .
$$

To prove that the $t_{i}(M)$ 's are left $D$-modules, we need the following important result.

Proposition 7 ([10]). If $0 \longrightarrow M^{\prime} \longrightarrow M \longrightarrow M^{\prime \prime} \longrightarrow 0$ is a short exact sequence of left modules over an Auslander regular ring $D$, then:

$$
j_{D}(M)=\inf \left\{j_{D}\left(M^{\prime}\right), j_{D}\left(M^{\prime \prime}\right)\right\} .
$$

Remark 8. If $\operatorname{ext}_{D}^{i}\left(M^{\prime}, D\right)=0$ and $\operatorname{ext}_{D}^{i}\left(M^{\prime \prime}, D\right)=0$ for $0 \leq i \leq j$, then Theorem 3 yields $\operatorname{ext}_{D}^{i}(M, D)=0$ for $0 \leq i \leq j$, which shows that $j_{D}(M) \geq \inf \left\{j_{D}\left(M^{\prime}\right), j_{D}\left(M^{\prime \prime}\right)\right\}$. Thus, the Auslander regularity condition is only used to prove the other inequality.

Let us now explain why $t_{i}(M)$ is a left $D$-module. If $m \in t_{i}(M)$ and $d \in D$, then $d m \in D m$, i.e., $D(d m) \subseteq D m$. Then, applying Proposition 7 to the following short exact sequence

$$
0 \longrightarrow D(d m) \longrightarrow D m \longrightarrow D m / D(d m) \longrightarrow 0,
$$

we get $j_{D}(D(d m)) \geq j_{D}(D m) \geq i$, i.e., $d m \in t_{i}(M)$. Let us now consider $m_{1}$ and $m_{2} \in t_{i}(M)$. Then, we have $m_{1}+m_{2} \in D m_{1}+D m_{2}$. Since $D\left(m_{1}+m_{2}\right) \subseteq D m_{1}+D m_{2}$, similarly as previously, Proposition 7 yields $j_{D}\left(D\left(m_{1}+m_{2}\right)\right) \geq j_{D}\left(D m_{1}+D m_{2}\right)$. Now, applying again Proposition 7 to the following two standard short exact sequences

$$
\begin{gathered}
0 \longrightarrow D m_{1} \cap D m_{2} \longrightarrow D m_{1} \oplus D m_{2} \longrightarrow D m_{1}+D m_{2} \longrightarrow 0 \\
0 \longrightarrow D m_{1} \longrightarrow D m_{1} \oplus D m_{2} \longrightarrow D m_{2} \longrightarrow 0
\end{gathered}
$$

(see, e.g., [50]), we then obtain the following inequality and equality

$$
\left\{\begin{array}{l}
j_{D}\left(D m_{1}+D m_{2}\right) \geq j_{D}\left(D m_{1} \oplus D m_{2}\right), \\
j_{D}\left(D m_{1} \oplus D m_{2}\right)=\inf \left\{j_{D}\left(D m_{1}\right), j_{D}\left(D m_{2}\right)\right\}=i,
\end{array}\right.
$$

which yields $j_{D}\left(D\left(m_{1}+m_{2}\right)\right) \geq i$, i.e., $m_{1}+m_{2} \in t_{i}(M)$.

If $M^{\prime}$ is a left $D$-submodule of $M$ such that $j_{D}\left(M^{\prime}\right) \geq i$ and if $m^{\prime} \in M^{\prime} \backslash\{0\}$, then applying Proposition 7 to the short exact sequence $0 \longrightarrow D m^{\prime} \longrightarrow M^{\prime} \longrightarrow M^{\prime} /\left(D m^{\prime}\right) \longrightarrow 0$, we get $j_{D}\left(D m^{\prime}\right) \geq j_{D}\left(M^{\prime}\right) \geq i$, i.e., $m^{\prime} \in t_{i}(M)$, and thus $M^{\prime} \subseteq t_{i}(M)$, which proves that $t_{i}(M)$ is the largest left $D$-submodule $L$ of $M$ ( $D$ is a noetherian ring) which satisfies $j_{D}(L) \geq i$.

Note that $t_{0}(M)=\left\{m \in M \mid j_{D}(D m) \geq 0\right\}=M$. Thus, the following filtration of $M$ holds:

$$
0=t_{n+1}(M) \subseteq t_{n}(M) \subseteq t_{n-1}(M) \subseteq \cdots \subseteq t_{1}(M) \subseteq t_{0}(M)=M .
$$

If $D$ is a domain, then using Corollary 1, we get $t_{1}(M)=t(M)$ since:

$$
m \in t(M) \Leftrightarrow \operatorname{ext}_{D}^{0}(D m, D)=0 \Leftrightarrow j_{D}(D m) \geq 1 \Leftrightarrow m \in t_{1}(M) .
$$

It can easily been seen that a module $M$ is $i$-pure iff $t_{i}(M)=M$ and $t_{i+1}(M)=0$.

Lemma 1. The left $D$-module $t_{i}(M) / t_{i+1}(M)$ is either zero or is $i$-pure. 
Proof. Let us suppose that $P=t_{i}(M) / t_{i+1}(M)$ is nonzero. Applying Proposition 7 to the short exact sequence $0 \longrightarrow t_{i+1}(M) \longrightarrow t_{i}(M) \longrightarrow P \longrightarrow 0$, we get $j_{D}(P) \geq j_{D}\left(t_{i}(M)\right) \geq i$, and thus $P \subseteq t_{i}(P) \subseteq P$, i.e., $t_{i}(P)=P$. Let us now check that $t_{i+1}(P)=0$, which will prove the result. Composing the two canonical projections $\alpha: t_{i}(M) \longrightarrow P=t_{i}(M) / t_{i+1}(M)$ and $\beta: P \longrightarrow P / t_{i+1}(P)$, we get the following commutative exact diagram:

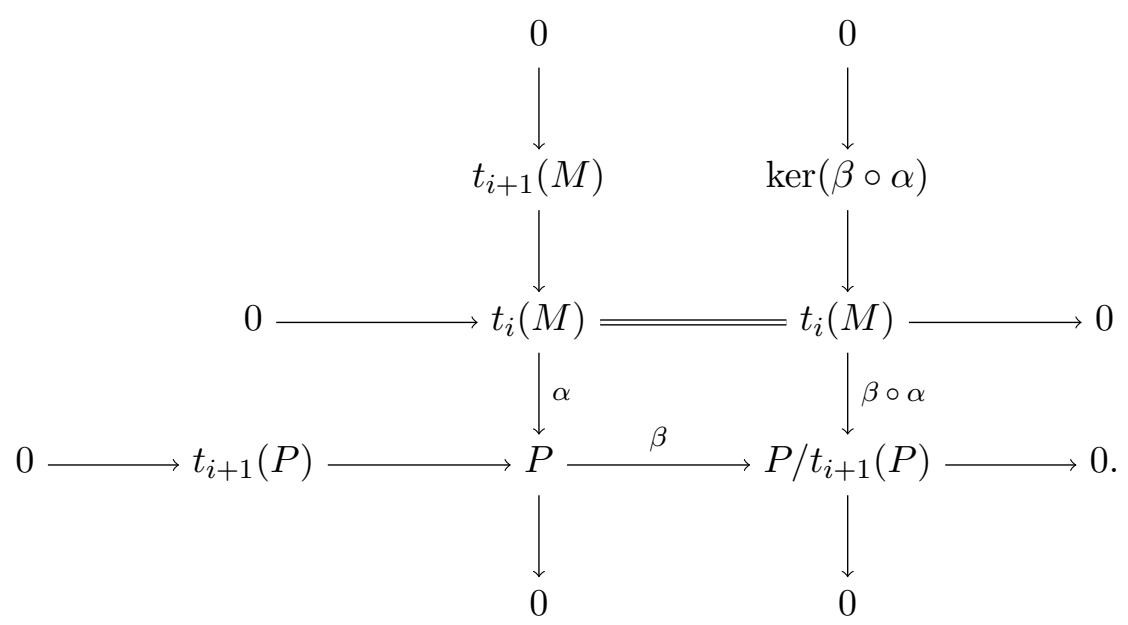

The snake lemma (see, e.g., [50]) then yields the following short exact sequence:

$$
0 \longrightarrow t_{i+1}(M) \longrightarrow \operatorname{ker}(\beta \circ \alpha) \longrightarrow t_{i+1}(P) \longrightarrow 0 .
$$

Using Proposition 7, we have $j_{D}(\operatorname{ker}(\beta \circ \alpha))=\inf \left\{j_{D}\left(t_{i+1}(M)\right), j_{D}\left(t_{i+1}(P)\right)\right\} \geq i+1$. Since $t_{i+1}(M) \subseteq \operatorname{ker}(\beta \circ \alpha) \subseteq t_{i}(M) \subseteq M$, we obtain $\operatorname{ker}(\beta \circ \alpha)=t_{i+1}(M)$, and thus $t_{i+1}(P)=0$ by the above short exact sequence.

According to Lemma 1, (22) is called the grade filtration (purity filtration) of $M$ (see [10]).

Theorem 9 ([9, 10, 11]). Let $D$ be a ring equipped with a filtration $\left\{D_{r}\right\}_{r \geq-1}\left(D_{-1}=0\right)$ such that the associated graded ring $\operatorname{gr}(D)=\bigoplus_{r \in \mathbb{N}} D_{r} / D_{r-1}$ satisfies the following three properties:

1. $\operatorname{gr}(D)$ is a commutative ring.

2. $\operatorname{gr}(D)$ is a noetherian ring.

3. $\operatorname{gr}(D)$ is a regular ring of pure dimension $d \in \mathbb{N}$, namely, $\operatorname{gld}\left(\operatorname{gr}(D)_{\mathfrak{m}}\right)$ is equal to $d$ for all localizations $\operatorname{gr}(D)_{\mathfrak{m}}$ of $\operatorname{gr}(D)$ at maximal ideals $\mathfrak{m}$ of $\operatorname{gr}(D)$.

Then, the following results hold:

1. $\operatorname{gld}\left(\operatorname{gr}(D)_{\mathfrak{m}}\right)$ is equal to the Krull dimension $\operatorname{Kdim}\left(\operatorname{gr}(D)_{\mathfrak{m}}\right)$ of the noetherian local ring $\operatorname{gr}(D)_{\mathfrak{m}}$, which also equal to the dimension $\operatorname{dim}_{\operatorname{gr}(D)_{\mathfrak{m}} / \mathfrak{m}}\left(\mathfrak{m} / \mathfrak{m}^{2}\right)$ of $\mathfrak{m} / \mathfrak{m}^{2}$ as a $\operatorname{gr}(D)_{\mathfrak{m}} / \mathfrak{m}$ vector space. This common value d for all maximal ideals $\mathfrak{m}$ of $\operatorname{gr}(D)$ is denoted by $\operatorname{dim}(D)$.

2. If $M \neq 0$ is a left $D$-module $M$, then the characteristic ideal $J(M)$ of $\operatorname{gr}(D)$, defined by

$$
J(M)=\sqrt{\operatorname{ann}_{\operatorname{gr}(D)}(\operatorname{gr}(M))}=\left\{a \in \operatorname{gr}(D) \mid \exists k \in \mathbb{N}: a^{k} \operatorname{gr}(M)=0\right\},
$$

does not depend on any good filtration of $M$ (e.g., if $M=\sum_{i=j}^{p} D y_{j}$ then $\left\{M_{r}\right\}_{r \in \mathbb{N}}$ defined by $M_{r}=\sum_{j=1}^{p} D_{r} y_{j}$ for all $r \in \mathbb{N}$ is a good filtration of $M$, and $\left.\operatorname{gr}(M)=\sum_{j=1}^{p} \operatorname{gr}(D) y_{j}\right)$. 
3. If the dimension of $M$ is defined by $\operatorname{dim}_{D}(M)=\operatorname{Kdim}(\operatorname{gr}(D) / J(M))$, then

$$
j_{D}(M)=\operatorname{dim}(D)-\operatorname{dim}_{D}(M),
$$

i.e., the codimension of $M$ is equal to the grade number of $M$.

A ring $D$ satisfying (23) is called a Cohen-Macaulay ring. A natural substitute for $\operatorname{dim}_{D}(\cdot)$ for more general $k$-algebras is the so-called Gel'fand-Kirillov dimension GKdim (see, e.g., [35]).

If $D$ satisfies the hypotheses of Theorem 9, then $\operatorname{dim}(D)=\operatorname{gld}(\operatorname{gr}(D))$ since we have $\operatorname{gld}(\operatorname{gr}(D))=\sup _{\mathfrak{m} \in \operatorname{Max}(\operatorname{gr}(D))} \operatorname{gld}\left(\operatorname{gr}(D)_{\mathfrak{m}}\right)$, where $\operatorname{Max}(\operatorname{gr}(D))$ is the set of the maximal ideals of $\operatorname{gr}(D)$ (see, e.g., [50]).

Example 5. If $k$ is a field of characteristic 0 and $A$ a differential field (namely, a field with a differential ring structure) of characteristic 0 (e.g., $\left.k, k\left(x_{1}, \ldots, x_{n}\right)\right)$, or $k\left[x_{1}, \ldots, x_{n}\right], k \llbracket x_{1}, \ldots, x_{n} \rrbracket$, $k^{\prime}\left\{x_{1}, \ldots, x_{n}\right\}$ where $k^{\prime}=\mathbb{R}$ or $\mathbb{C}$, then the ring $D=A\left\langle\partial_{1}, \ldots, \partial_{n}\right\rangle$ of PD operators with coefficients in $A$ is Auslander regular and Cohen-Macaulay (see [9, 10, 11]). In particular, if $\left\{D_{i}\right\}_{i \geq-1}$ is the order filtration of $D$, namely $D_{i}$ is the $A$-submodule of $D$ formed by the PD operators of order less than or equal to $i$, and $\chi_{i}$ is the class of $\partial_{i}$ in $D_{1} / D_{0}$, then $\operatorname{gr}(D)=A\left[\chi_{1}, \ldots, \chi_{n}\right]$. Thus, if $A$ is a differential field of characteristic 0 (e.g., $k, k\left(x_{1}, \ldots, x_{n}\right)$ ), then $\operatorname{dim}(D)=n$, and if $A=k\left[x_{1}, \ldots, x_{n}\right], k \llbracket x_{1}, \ldots, x_{n} \rrbracket$, or $k^{\prime}\left\{x_{1}, \ldots, x_{n}\right\}$, then $\operatorname{dim}(A)=n$ and $\operatorname{dim}(D)=2 n$.

Corollary 3 ([9, 10, 11]). Let $D$ be an Auslander regular ring and a Cohen-Macaulay ring, and $M$ a nonzero finitely generated left D-module. Then, we have:

1. $\operatorname{dim}_{D}\left(\operatorname{ext}_{D}^{i}(M, D)\right) \leq \operatorname{dim}(D)-i$.

2. $\operatorname{dim}_{D}\left(\operatorname{ext}_{D}^{j_{D}(M)}(M, D)\right)=\operatorname{dim}(D)-j_{D}(M)$.

3. If $\operatorname{ext}_{D}^{i}\left(\operatorname{ext}_{D}^{i}(M, D), D\right) \neq 0$, then $\operatorname{dim}_{D}\left(\operatorname{ext}_{D}^{i}\left(\operatorname{ext}_{D}^{i}(M, D), D\right)\right)=\operatorname{dim}(D)-i$.

4. If $M$ is an i-pure left $D$-module, then $\operatorname{dim}_{D}(M)=\operatorname{dim}(D)-i$.

If $D$ is an Auslander regular ring with $\operatorname{gld}(D)=n$, then a nonzero finitely generated left $D$-module $M$ is called holonomic (resp., subholonomic) if $j_{D}(M)=n$ (resp., $j_{D}(M) \geq n-1$ ). It is convenient to assume that $M=0$ is also holonomic so that $M$ is holonomic if $j_{D}(M) \geq n$. If $D$ is also a Cohen-Macaulay ring, then $M \neq 0$ is holonomic (resp., subholonomic) iff $\operatorname{dim}_{D}(M)=$ $\operatorname{dim}(D)-n$ (resp., $\operatorname{dim}_{D}(M) \leq \operatorname{dim}(D)-n+1$ ). In particular, if $D$ is one of the rings of PD operators defined in Example 5, then we find again the classical definitions of holonomic and subholonomic modules over a ring of PD operators (see, e.g., [9, 10, 11, 33]).

Let us state a few remarks on holonomic modules. If $0 \longrightarrow M^{\prime} \longrightarrow M \longrightarrow M^{\prime \prime} \longrightarrow 0$ is a short exact sequence and $j_{D}\left(M^{\prime}\right)=j_{D}\left(M^{\prime \prime}\right)=i$, then $j_{D}(M)=i$ by Proposition 7. In particular, if $M^{\prime}$ and $M^{\prime \prime}$ are two holonomic left $D$-modules, so is $M$. The converse result also holds since Proposition 7 and $j_{D}(M) \geq n$ yield $j_{D}\left(M^{\prime}\right) \geq n$ and $j_{D}\left(M^{\prime \prime}\right) \geq n$. Thus, $M$ is a holonomic left $D$-module iff $M^{\prime}$ and $M^{\prime \prime}$ are two holonomic left $D$-modules. Finally, a simple module (i.e., a module containing no nontrivial submodules) left $A_{n}(k)$-module is not necessarily holonomic as shown in [52]. But, a simple module over an Auslander regular ring $D$ is pure. 


\section{Grade filtration}

The goal of the section is to show how the grade filtration $(22)$ of a finitely generated left module $M$ over an Auslander regular ring $D$ can be explicitly computed. Since we are motivated by developing an effective algorithm which can be implemented in computer algebra systems, in what follows, we shall only use free resolutions of modules and not the more general projective resolutions. This extension can easily be done and it is left to the interested reader.

Let $D$ be a noetherian regular ring, i.e., a noetherian domain $D$ with a finite global dimension $\operatorname{gld}(D)=n$, and $M$ a finitely generated left $D$-module. Let us consider a free resolution of $M$ :

$$
0 \longleftarrow M \stackrel{\pi}{\longleftarrow} D^{1 \times p_{0}} \stackrel{. R_{1}}{\longleftarrow} D^{1 \times p_{1}} \stackrel{. R_{2}}{\longleftarrow} \ldots \stackrel{. R_{i-1}}{\longleftarrow} D^{1 \times p_{i-1}} \stackrel{. R_{i}}{\longleftarrow} D^{1 \times p_{i}} \stackrel{R_{i+1}}{\longleftarrow} \ldots
$$

Using (7) and Proposition 3, the defects of exactness of the following complex

$$
0 \longrightarrow D^{p_{0}} \stackrel{R_{1} \cdot}{\longrightarrow} D^{p_{1}} \stackrel{R_{2} \cdot}{\longrightarrow} \ldots \stackrel{R_{i-1} \cdot}{\longrightarrow} D^{p_{i-1}} \stackrel{R_{i \cdot}}{\longrightarrow} D^{p_{i}} \stackrel{R_{i+1} \cdot}{\longrightarrow} D^{p_{i+1}} \stackrel{R_{i+2 \cdot}}{\longrightarrow} \ldots
$$

are the right $D$-modules defined by:

$$
\left\{\begin{array}{l}
\operatorname{ext}_{D}^{0}(M, D) \cong \operatorname{ker}_{D}\left(R_{1} \cdot\right) \\
\operatorname{ext}_{D}^{i}(M, D) \cong \operatorname{ker}_{D}\left(R_{i+1} \cdot\right) /\left(R_{i} D^{p_{i-1}}\right), \quad 1 \leq i \leq n, \\
\operatorname{ext}_{D}^{i}(M, D)=0, \quad i>n .
\end{array}\right.
$$

To characterize the $\operatorname{ext}_{D}^{i}(M, D)$ 's for all $0 \leq i \leq n$, we need to study $\operatorname{ker}_{D}\left(R_{i+1}\right.$.). For $1 \leq k \leq n+1$, considering the beginning of a free resolution of the finitely generated right $D$-module $\operatorname{ker}_{D}\left(R_{k}\right.$.), we obtain the following long exact sequence of right $D$-modules

$$
D^{p_{(-1) k}} \stackrel{R_{0 k} \cdot}{\longrightarrow} D^{p_{0 k}} \stackrel{R_{1 k} \cdot}{\longrightarrow} D^{p_{1 k}} \stackrel{R_{2 k} .}{\longrightarrow} \ldots \stackrel{R_{(k-1) k}}{\longrightarrow} D^{p_{(k-1) k}} \stackrel{R_{k k} \cdot}{\longrightarrow} D^{p_{k k}} \stackrel{\kappa_{k k}}{\longrightarrow} N_{k k} \longrightarrow 0,
$$

where for $k$ from 1 to $n+1$, we have set $R_{k k}=R_{k}, p_{k k}=p_{k}, p_{(k-1) k}=p_{k-1}=p_{(k-1)(k-1)}$ and:

$$
N_{k k}=\operatorname{coker}_{D}\left(R_{k k} .\right)=D^{p_{k k}} /\left(R_{k k} D^{p_{(k-1) k}}\right) .
$$

Let us explain why this choice of the notations is natural. If we consider a squared-line paper sheet where each square has coordinates $(j, k) \in \mathbb{N}^{2}$, and if the long exact sequence (27) is placed at $k^{\text {th }}$ level with $D^{p_{j k}}$ at position $(j, k)$, then the horizontal arrow of the right $D$-homomorphism $R_{j k}$. arrives at $D^{p_{j k}}$ with $j \leq k$ (a good mnemonic device). For instance, the first three horizontal exact sequences can be arranged as follows:

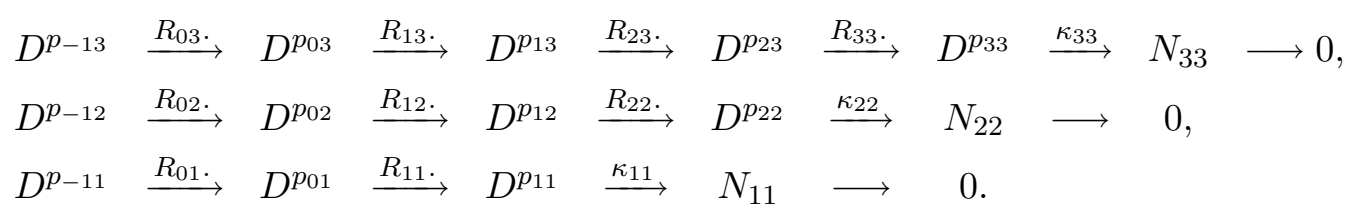

Since 25 is a complex, $R_{k k} R_{(k-1)(k-1)}=R_{k} R_{k-1}=0$ for all $k=2, \ldots, n+1$, and thus

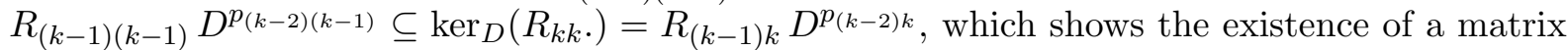
$F_{(k-2) k} \in D^{p_{(k-2) k} \times p_{(k-2)(k-1)}}$ such that:

$$
\forall k=2, \ldots, n+1, \quad R_{(k-1)(k-1)}=R_{(k-1) k} F_{(k-2) k} .
$$


Then, using (28), we get $R_{(k-1) k} F_{(k-2) k} R_{(k-2)(k-1)}=R_{(k-1)(k-1)} R_{(k-2)(k-1)}=0$, i.e.,

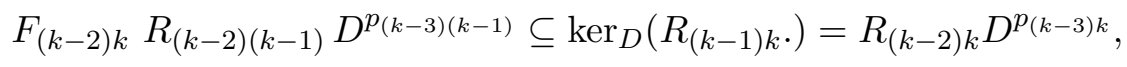

and thus, there exists a matrix $F_{(k-3) k} \in D^{p_{(k-3) k} \times p_{(k-3)(k-1)}}$ such that:

$$
\forall k=2, \ldots, n+1, \quad F_{(k-2) k} R_{(k-2)(k-1)}=R_{(k-2) k} F_{(k-3) k} .
$$

Similarly, we can show that for $k=3, \ldots, n+1$, there exist matrices $F_{(k-j) k} \in D^{p_{(k-j) k} \times p_{(k-j)(k-1)}}$ with $j=3, \ldots, k$ such that:

$$
F_{(k-j) k} R_{(k-j)(k-1)}=R_{(k-j) k} F_{(k-j-1) k} .
$$

Let us denote by:

$$
R_{00}=0, \quad N_{00}=D^{p_{00}} / 0 \cong D^{p_{00}}, \quad p_{01}=p_{00}, \quad p_{-10}=0 .
$$

Using (27), 28), 29), (30) and (31), we get the following commutative diagram formed by $n+2$ horizontal exact sequences (where to reduce the size of the diagram, we set $m=n+1$ ):

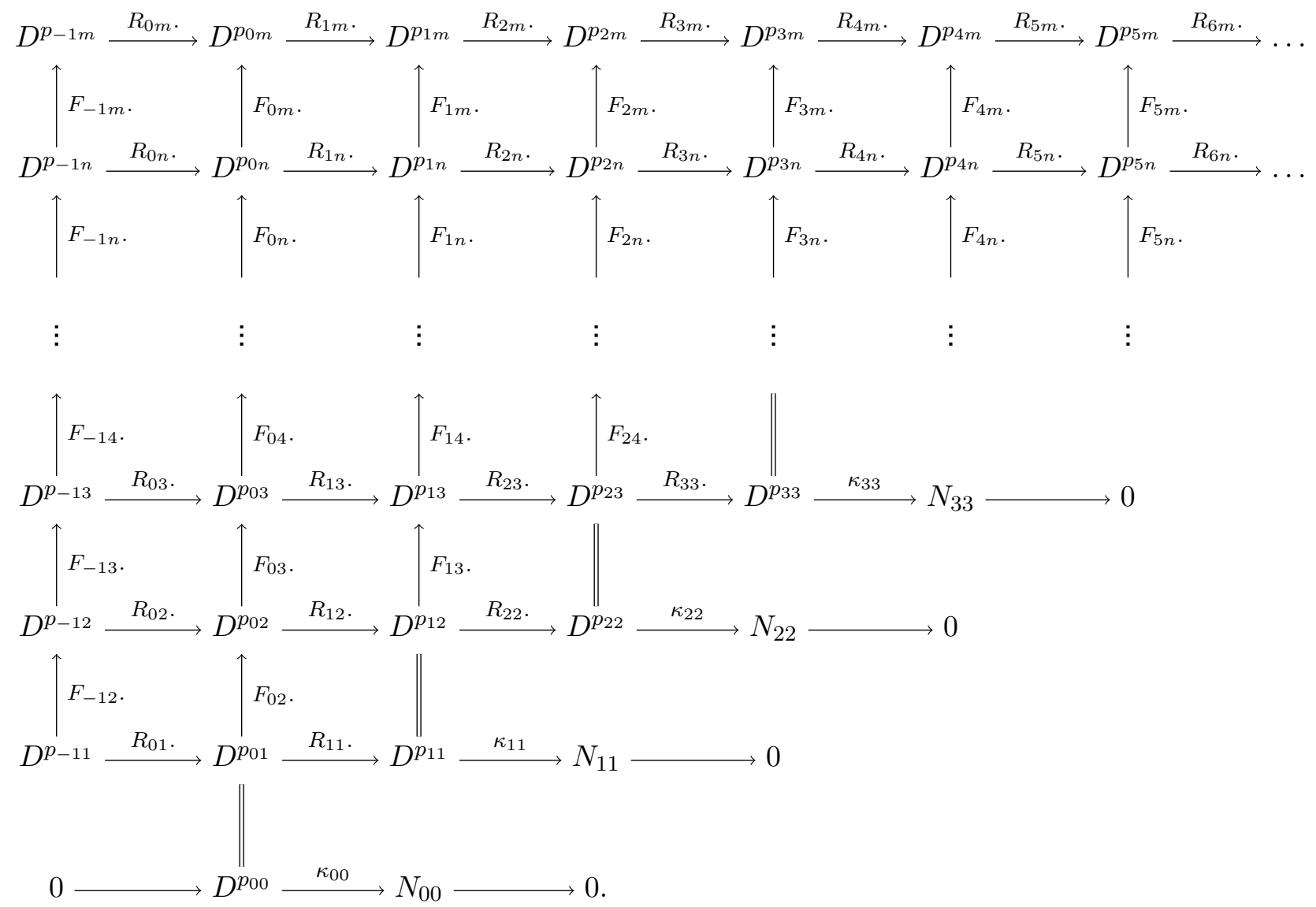

Now, if we denote by $N_{(k-j) k}$ the finitely presented right $D$-module defined by

$$
N_{(k-j) k}=\operatorname{coker}_{D}\left(R_{(k-j) k} .\right)=D^{p_{(k-j) k}} /\left(R_{(k-j) k} D^{\left.p_{(k-j-1) k}\right)},\right.
$$


then, (32) can be truncated to get the following commutative diagram formed by horizontal exact sequences:

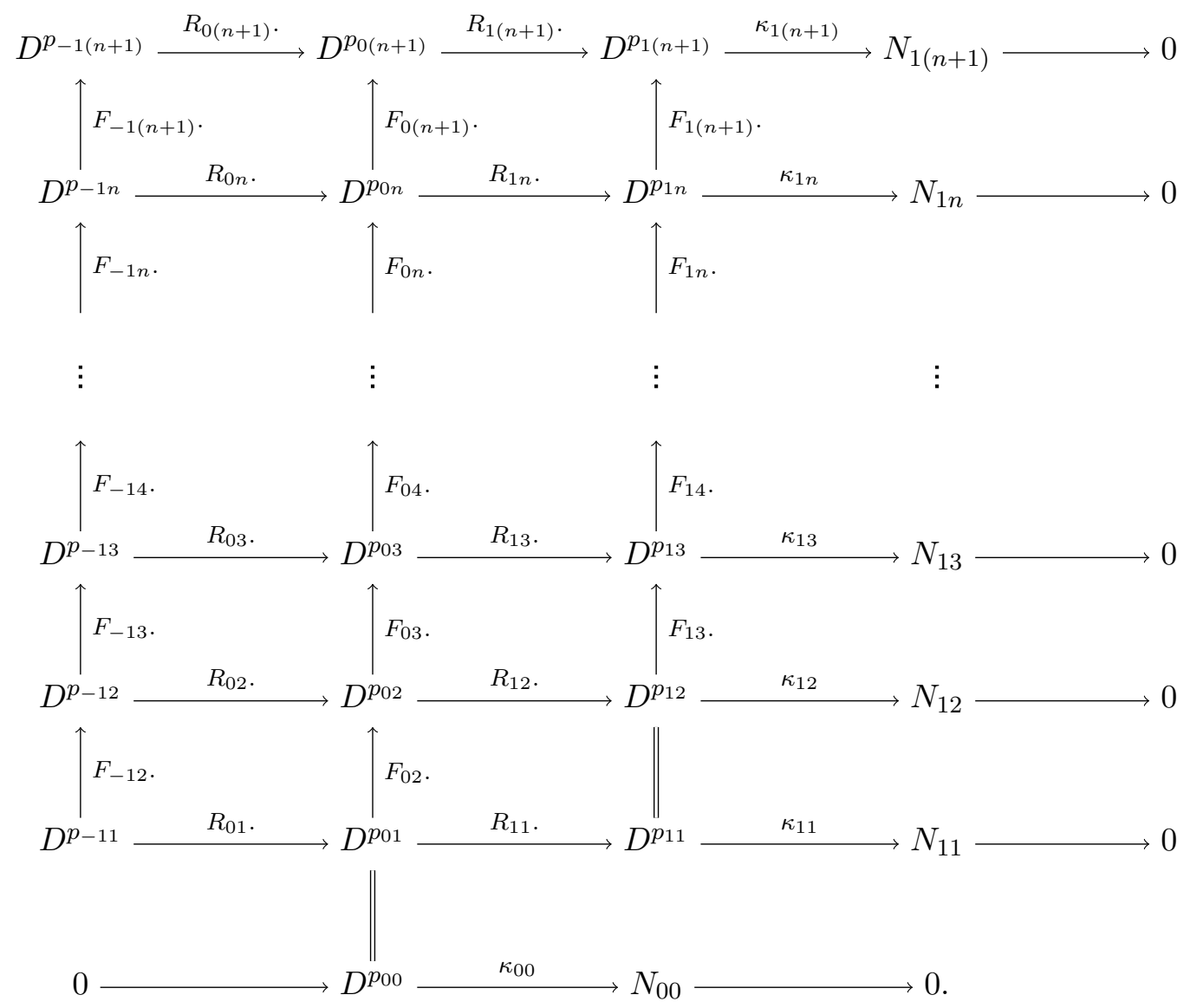

For $k=1, \ldots, n+1$ and $j=0, \ldots, k-1$, using the exactness of the following complex

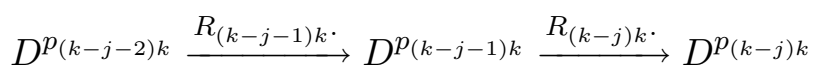

at $D^{p_{(k-j-1) k}}$, we get $N_{(k-j-1) k}=\operatorname{coker}_{D}\left(R_{(k-j-1) k}.\right) \cong \operatorname{im}_{D}\left(R_{(k-j) k}.\right)$ which, when combined with the short exact sequence $0 \longrightarrow \operatorname{im}_{D}\left(R_{(k-j) k}.\right) \longrightarrow D^{p_{(k-j) k}} \stackrel{\kappa_{(k-j) k}}{\longrightarrow} N_{(k-j) k} \longrightarrow 0$, yields the following short exact sequence of right $D$-modules:

$$
0 \longrightarrow N_{(k-j-1) k} \longrightarrow D^{p_{(k-j) k}} \longrightarrow N_{(k-j) k} \longrightarrow 0 .
$$

Using (26), we obtain the following characterization of the right $D$-modules $\operatorname{ext}_{D}^{i}(M, D)$ 's:

$$
\left\{\begin{array}{lr}
\operatorname{ext}_{D}^{i}(M, D) \cong \operatorname{ker}_{D}\left(R_{(i+1)(i+1)} \cdot\right) / \operatorname{im}_{D}\left(R_{i i} .\right)=\left(R_{i(i+1)} D^{\left.p_{(i-1)(i+1)}\right)}\right) /\left(R_{i i} D^{p_{(i-1) i}}\right), & 0 \leq i \leq n, \\
\operatorname{ext}_{D}^{i}(M, D)=0, & i>n .
\end{array}\right.
$$

Since $N_{i i}=D^{p_{i i}} /\left(R_{i i} D^{p_{(i-1) i}}\right), N_{i(i+1)}=D^{p_{(i+1)}} /\left(R_{i(i+1)} D^{\left.p_{(i-1)(i+1)}\right)}, p_{i(i+1)}=p_{i i}\right.$, and $N_{00}=$ $D^{p_{00}},(35)$ and the third isomorphism theorem of module theory (see, e.g., [50]) yield the following short exact sequence of right $D$-modules:

$$
0 \longrightarrow \operatorname{ext}_{D}^{i}(M, D) \longrightarrow N_{i i} \longrightarrow N_{i(i+1)} \longrightarrow 0, \quad i=0, \ldots, n .
$$


Applying the contravariant left exact functor $\operatorname{hom}_{D}(\cdot, D)$ to the short exact sequence of (36) and using Theorem 3, we obtain the following long exact sequences:

$$
\begin{aligned}
& 0 \quad \longrightarrow \quad \operatorname{ext}_{D}^{0}\left(N_{01}, D\right) \quad \longrightarrow \operatorname{ext}_{D}^{0}\left(N_{00}, D\right) \longrightarrow \operatorname{ext}_{D}^{0}\left(\operatorname{ext}_{D}^{0}(M, D), D\right) \\
& \cdots \quad \longrightarrow \quad \begin{array}{cll}
\operatorname{ext}_{D}^{1}\left(N_{01}, D\right) & \longrightarrow \operatorname{ext}_{D}^{1}\left(N_{00}, D\right), \\
\operatorname{ext}_{D}^{i-1}\left(N_{i(i+1)}, D\right) \longrightarrow \operatorname{ext}_{D}^{i-1}\left(N_{i i}, D\right) \longrightarrow \operatorname{ext}_{D}^{i-1}\left(\operatorname{ext}_{D}^{i}(M, D), D\right)
\end{array} \\
& \longrightarrow \operatorname{ext}_{D}^{i}\left(N_{i(i+1)}, D\right) \longrightarrow \operatorname{ext}_{D}^{i}\left(N_{i i}, D\right) \longrightarrow \operatorname{ext}_{D}^{i}\left(\operatorname{ext}_{D}^{i}(M, D), D\right) \\
& \stackrel{\tau_{i+1}}{\longrightarrow} \operatorname{ext}_{D}^{i+1}\left(N_{i(i+1)}, D\right) \longrightarrow \operatorname{ext}_{D}^{i+1}\left(N_{i i}, D\right) \longrightarrow \quad \ldots, \quad i=1, \ldots, n .
\end{aligned}
$$

In what follows, we shall assume that $D$ satisfies the following property

$$
\forall i \geq 1, \quad \operatorname{ext}_{D}^{i-1}\left(\operatorname{ext}_{D}^{i}(M, D), D\right)=0,
$$

for all finitely generated left $D$-modules $M$. In particular, by Remark 7, this condition holds if $D$ is an Auslander regular ring (see Definition 8).

We note that $\operatorname{ext}_{D}^{1}\left(N_{00}, D\right)$ is reduced to 0 since $N_{00}=D^{p_{00}}$ is a free, and thus a projective right $D$-module (see Remark 22). Using (38), the above long exact sequences then yield the following long exact sequences of left $D$-modules:

$$
\begin{gathered}
0 \longrightarrow \operatorname{ext}_{D}^{0}\left(N_{01}, D\right) \longrightarrow \operatorname{ext}_{D}^{0}\left(N_{00}, D\right) \longrightarrow \operatorname{ext}_{D}^{0}\left(\operatorname{ext}_{D}^{0}(M, D), D\right) \longrightarrow \operatorname{ext}_{D}^{1}\left(N_{01}, D\right) \longrightarrow 0, \\
\left.0 \longrightarrow \operatorname{ext}_{D}^{i}\left(N_{i(i+1)}, D\right) \longrightarrow \operatorname{ext}_{D}^{i}\left(N_{i i}, D\right) \longrightarrow \operatorname{ext}_{D}^{i}(M, D), D\right), \quad i=1, \ldots, n .
\end{gathered}
$$

Applying Proposition 1 to (34) for $k=i+1$ and $j=0, \ldots, i-1$, i.e., to the short exact sequence $0 \longrightarrow N_{(i-j)(i+1)} \longrightarrow D^{p_{(i-j+1)(i+1)}} \longrightarrow N_{(i-j+1)(i+1)} \longrightarrow 0$, we obtain:

$$
\forall i=1, \ldots, n, \quad \operatorname{ext}_{D}^{i+1}\left(N_{(i+1)(i+1)}, D\right) \cong \operatorname{ext}_{D}^{i}\left(N_{i(i+1)}, D\right) \cong \ldots \cong \operatorname{ext}_{D}^{1}\left(N_{1(i+1)}, D\right) .
$$

Similarly, applying Proposition 1 to (34) for $k=i+1$ and $j=0$ gives:

$$
\operatorname{ext}_{D}^{i+2}\left(N_{(i+1)(i+1)}, D\right) \cong \operatorname{ext}_{D}^{i+1}\left(N_{i(i+1)}, D\right) .
$$

Applying Proposition 1 to the above short exact sequence with $i=0$ and $j=0$, we get:

$$
\operatorname{ext}_{D}^{2}\left(N_{11}, D\right) \cong \operatorname{ext}_{D}^{1}\left(N_{01}, D\right) .
$$

Thus, the first long exact sequence of (39) yields the following one

$$
0 \longrightarrow \operatorname{ext}_{D}^{0}\left(N_{01}, D\right) \stackrel{\gamma_{10}}{\longrightarrow} \operatorname{ext}_{D}^{0}\left(N_{00}, D\right) \stackrel{\gamma_{00}}{\longrightarrow} \operatorname{ext}_{D}^{0}\left(\operatorname{ext}_{D}^{0}(M, D), D\right) \longrightarrow \operatorname{ext}_{D}^{2}\left(N_{11}, D\right) \longrightarrow 0
$$

and $(39)$ and $(40)$ yield the following exact sequence of left $D$-modules

$$
0 \longrightarrow \operatorname{ext}_{D}^{i+1}\left(N_{(i+1)(i+1)}, D\right) \stackrel{\gamma_{(i+1) i}}{\longrightarrow} \operatorname{ext}_{D}^{i}\left(N_{i i}, D\right) \stackrel{\gamma_{i i}}{\longrightarrow} \operatorname{ext}_{D}^{i}\left(\operatorname{ext}_{D}^{i}(M, D), D\right) \longrightarrow \operatorname{coker} \gamma_{i i} \longrightarrow 0,
$$

where:

$$
\forall i=1, \ldots, n, \quad \text { coker } \gamma_{i i} \subseteq \operatorname{ext}_{D}^{i+1}\left(N_{i(i+1)}, D\right) \cong \operatorname{ext}_{D}^{i+2}\left(N_{(i+1)(i+1)}, D\right) .
$$

Hence, if we introduce the following finitely generated left $D$-modules

$$
\forall i=0, \ldots, n+1, \quad T_{i} \triangleq \operatorname{ext}_{D}^{i}\left(N_{i i}, D\right),
$$

then 43 can be rewritten as the following exact sequences:

$$
0 \longrightarrow T_{i+1} \stackrel{\gamma_{(i+1) i}}{\longrightarrow} T_{i} \stackrel{\gamma_{i i}}{\longrightarrow} \operatorname{ext}_{D}^{i}\left(\operatorname{ext}_{D}^{i}(M, D), D\right) \longrightarrow \operatorname{coker} \gamma_{i i} \longrightarrow 0, \quad i=1, \ldots, n .
$$


Remark 9. If $D$ is an Auslander regular ring, then using (45) and Remark 7, $T_{i}$ is either zero or $j_{D}\left(T_{i}\right) \geq i$. Moreover, according to 3 of Theorem 8 , $\operatorname{ext}_{D}^{i}\left(\operatorname{ext}_{D}^{i}(M, D), D\right)$ is either zero or is $i$-pure. In particular, $T_{i} / \gamma_{(i+1) i}\left(T_{i+1}\right)$ is a left $D$-submodule of $\operatorname{ext}_{D}^{i}\left(\operatorname{ext}_{D}^{i}(M, D), D\right)$, and thus it is either zero or is $i$-pure by Remark 7. Finally, using Remark 7 and 44, we find that coker $\gamma_{i i}$ is either zero or $j_{D}\left(\operatorname{coker} \gamma_{i i}\right)=j_{D}\left(\operatorname{ext}_{D}^{i+2}\left(N_{(i+1)(i+1)}, D\right)\right) \geq i+2$.

Using (40), up to isomorphism, the left $D$-modules $T_{i}$ 's are the defects of exactness at $D^{1 \times p_{0 i}}$ of the horizontal complexes of the following commutative diagram (marked in red)

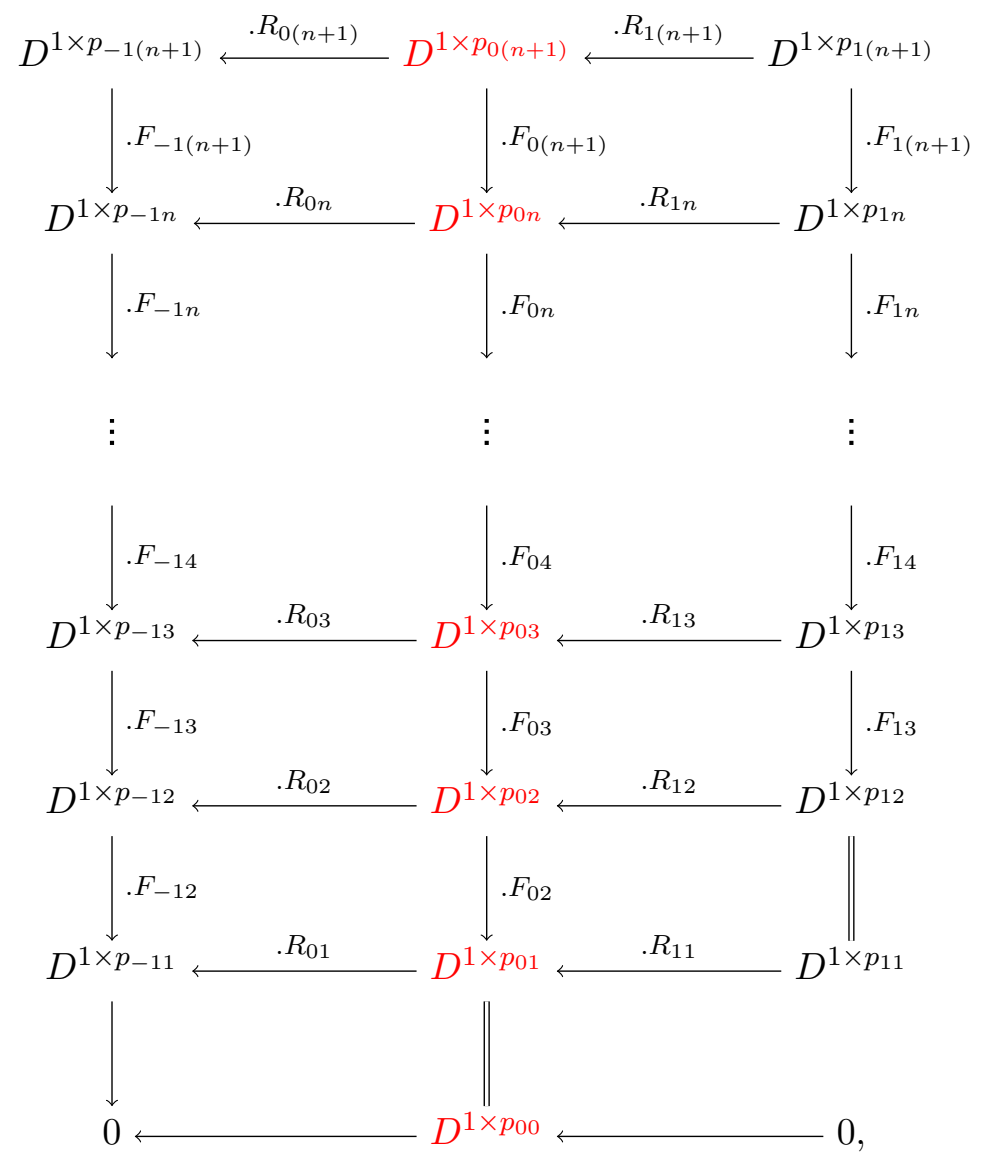

i.e., we have:

$$
T_{0}=D^{1 \times p_{00}}, \quad T_{i}=\operatorname{ker}_{D}\left(. R_{0 i}\right) / \operatorname{im}_{D}\left(. R_{1 i}\right), \quad i=1, \ldots, n+1 .
$$

If $\rho_{i}: \operatorname{ker}_{D}\left(. R_{0 i}\right) \longrightarrow T_{i}=\operatorname{ker}_{D}\left(. R_{0 i}\right) /\left(D^{1 \times p_{1 i}} R_{1 i}\right)$ is the canonical projection onto the $D$ module $T_{i}$ for $i=1, \ldots, n+1$, then $\gamma_{(i+1) i} \in \operatorname{hom}_{D}\left(T_{i+1}, T_{i}\right)$ (see 460) is defined by:

$$
\forall \lambda \in \operatorname{ker}_{D}\left(. R_{0(i+1)}\right), \quad \gamma_{(i+1) i}\left(\rho_{i+1}(\lambda)\right)=\rho_{i}\left(\lambda F_{0(i+1)}\right), \quad i=1, \ldots, n .
$$


The inclusion $\operatorname{ker}_{D}\left(. R_{01}\right) \subseteq D^{1 \times p_{01}}$ yields the following commutative exact diagram

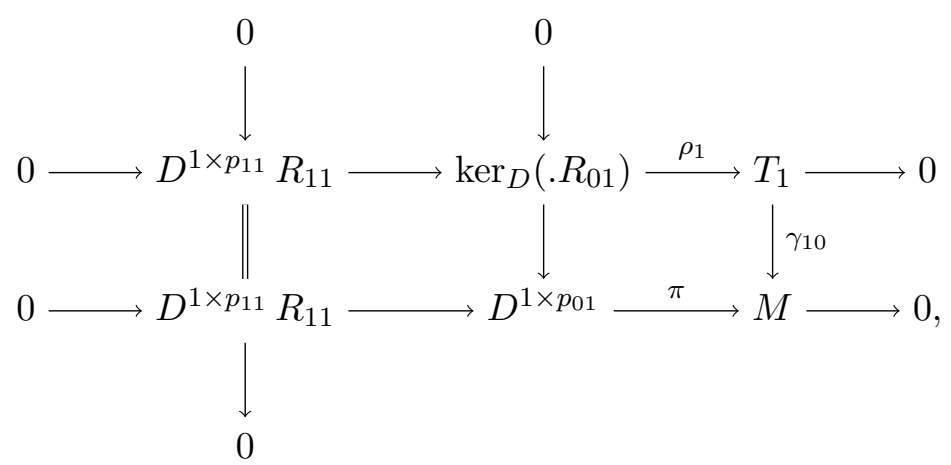

where $\gamma_{10} \in \operatorname{hom}_{D}\left(T_{1}, M\right)$ is defined by

$$
\forall \lambda \in \operatorname{ker}_{D}\left(. R_{01}\right), \quad \gamma_{10}\left(\rho_{1}(\lambda)\right)=\pi(\lambda),
$$

and $\pi$ is the canonical projection onto $M=D^{1 \times p_{01}} /\left(D^{1 \times p_{11}} R_{11}\right)$, i.e., $\gamma_{10}=\mathrm{id}_{T_{1}}$. In particular, $\gamma_{10}$ is injective. Moreover, using $T_{1}=\operatorname{ker}_{D}\left(. R_{01}\right) /\left(D^{1 \times p_{11}} R_{11}\right) \subseteq M=D^{1 \times p_{01}} /\left(D^{1 \times p_{11}} R_{11}\right)$, the third isomorphism theorem of module theory (see, e.g., [50]) gives:

$$
M / T_{1} \cong D^{1 \times p_{01}} / \operatorname{ker}_{D}\left(. R_{01}\right) .
$$

Finally, if $D$ is a domain, then 1 of Theorem 5 shows that $T_{1}=t(M)$ and $M / T_{1}=M / t(M)$.

Let us now study the long exact sequences (42) and (46) for $i=n-1, n$.

A right $D$-module analogous of Theorem 1 shows that $\operatorname{ext}_{D}^{0}\left(N_{01}, D\right) \cong \operatorname{ker}_{D}\left(. R_{01}\right)$. Using (31), $T_{0}=\operatorname{ext}_{D}^{0}\left(N_{00}, D\right)=\operatorname{hom}_{D}\left(D^{p_{00}}, D\right) \cong D^{1 \times p_{00}}=D^{1 \times p_{01}}$ (see (47)). The long exact sequence 42 then becomes the following one:

$$
0 \longrightarrow \operatorname{ker}_{D}\left(. R_{01}\right) \stackrel{\gamma_{10}}{\longrightarrow} D^{1 \times p_{01}} \stackrel{\gamma_{00}}{\longrightarrow} \operatorname{ext}_{D}^{0}\left(\operatorname{ext}_{D}^{0}(M, D), D\right) \longrightarrow \operatorname{ext}_{D}^{2}\left(N_{11}, D\right) \longrightarrow 0 .
$$

Proposition 3, $\operatorname{gld}(D)=n$ and 44 yield coker $\gamma_{(n-1)(n-1)} \subseteq \operatorname{ext}_{D}^{n+1}\left(N_{n n}, D\right)=0$, i.e., coker $\gamma_{(n-1)(n-1)}=0$. Thus, setting $i=n-1$ in $(46)$, we get the following short exact sequence

$$
0 \longrightarrow T_{n} \stackrel{\gamma_{n(n-1)}}{\longrightarrow} T_{n-1} \stackrel{\gamma_{(n-1)(n-1)}}{\longrightarrow} \operatorname{ext}_{D}^{n-1}\left(\operatorname{ext}_{D}^{n-1}(M, D), D\right) \longrightarrow 0,
$$

which shows that:

$$
T_{n-1} /\left(\gamma_{n(n-1)}\left(T_{n}\right)\right) \cong \operatorname{ext}_{D}^{n-1}\left(\operatorname{ext}_{D}^{n-1}(M, D), D\right) .
$$

Proposition $3, \operatorname{gld}(D)=n$ and $(44)$ imply that coker $\gamma_{n n} \subseteq \operatorname{ext}_{D}^{n+2}\left(N_{(n+1)(n+1)}, D\right)=0$, i.e., coker $\gamma_{n n}=0$. By Proposition 3, we also have:

$$
T_{n+1}=\operatorname{ext}_{D}^{n+1}\left(N_{(n+1)(n+1)}, D\right)=0 .
$$

Thus, setting $i=n$ in (46), we obtain the following short exact sequence

$$
0 \longrightarrow T_{n} \stackrel{\gamma_{n n}}{\longrightarrow} \operatorname{ext}_{D}^{n}\left(\operatorname{ext}_{D}^{n}(M, D), D\right) \longrightarrow 0,
$$

which shows that:

$$
T_{n} \cong \operatorname{ext}_{D}^{n}\left(\operatorname{ext}_{D}^{n}(M, D), D\right)
$$


Therefore, the following exact sequences of left $D$-modules hold

$\begin{array}{ccccccc} & 0 & \longrightarrow & T_{n} & \stackrel{\gamma_{n n}}{\longrightarrow} & \operatorname{ext}_{D}^{n}\left(\operatorname{ext}_{D}^{n}(M, D), D\right) & \longrightarrow 0, \\ 0 \longrightarrow & T_{n} & \stackrel{\gamma_{n(n-1)}}{\longrightarrow} & T_{n-1} & \longrightarrow & \operatorname{coker} \gamma_{n(n-1)} & \longrightarrow 0, \\ \vdots & & \vdots & & \vdots & \\ 0 \longrightarrow & T_{i} & \stackrel{\gamma_{i(i-1)}}{\longrightarrow} & T_{i-1} & \longrightarrow & \operatorname{coker} \gamma_{i(i-1)} & \longrightarrow 0, \\ \vdots & & \vdots & & \vdots & \\ 0 \longrightarrow & T_{2} & \stackrel{\gamma_{21}}{\longrightarrow} & T_{1} & \longrightarrow & \operatorname{coker} \gamma_{21} & \longrightarrow 0, \\ 0 \longrightarrow & T_{1} & \stackrel{\gamma_{10}}{\longrightarrow} & M & \stackrel{\rho}{\longrightarrow} & M / T_{1} & \longrightarrow 0, \\ 0 \longrightarrow \operatorname{ker}_{D}\left(. R_{01}\right) & \longrightarrow & D^{1 \times p_{01}} & \stackrel{\pi^{\prime}}{\longrightarrow} & M / T_{1} & \longrightarrow 0, \\ 0 \longrightarrow & M / T_{1} & \longrightarrow & \operatorname{ext}_{D}^{0}\left(\operatorname{ext}_{D}^{0}(M, D), D\right) & \longrightarrow & \operatorname{ext}_{D}^{2}\left(N_{11}, D\right) & \longrightarrow 0,\end{array}$

where:

$$
\forall i=2, \ldots, n, \quad \operatorname{coker} \gamma_{i(i-1)} \subseteq \operatorname{ext}_{D}^{i}\left(\operatorname{ext}_{D}^{i}(M, D), D\right) .
$$

Now, since the $\gamma_{i(i-1)}$ 's are injective left $D$-homomorphisms and $\gamma_{10}=\mathrm{id}_{T_{1}}$, we can define the following sequence $\left\{M_{i}\right\}_{i=0, \ldots, n}$ of left $D$-submodules of $M$ as follows:

$M_{0}=M, \quad M_{1}=\gamma_{10}\left(T_{1}\right)=T_{1}, \quad \forall i=2, \ldots, n, \quad M_{i}=\left(\gamma_{10} \circ \gamma_{21} \circ \gamma_{32} \circ \cdots \circ \gamma_{i(i-1)}\right)\left(T_{i}\right) \cong T_{i}$.

Using (48) and (49), the left $D$-module $M_{i}$ can be explicitly characterized by:

$$
\forall i=1, \ldots, n, \quad M_{i}=\pi\left(\operatorname{ker}_{D}\left(. R_{0 i}\right)\left(F_{0 i} \ldots F_{02}\right)\right) .
$$

The inclusion $\gamma_{i(i-1)}\left(T_{i}\right) \subseteq T_{i-1}$ yields $M_{i} \subseteq M_{i-1}$, and we get the following filtration of $M$ :

$$
0=M_{n+1} \subseteq M_{n} \subseteq M_{n-1} \subseteq \cdots \subseteq M_{2} \subseteq M_{1} \subseteq M_{0}=M
$$

Remark 10. Let us explain why the left $D$-modules $M_{i}$ 's depend only on $M$ and not on the free resolution (24) of $M$. Using Remark 3, the Auslander transpose right $D$-module $N_{i i}=$ $D^{p_{i i}} /\left(R_{i i} D^{p_{(i-1) i}}\right)$ of the left $D$-module $\operatorname{coker}_{D}\left(. R_{i i}\right)=D^{1 \times p_{i i}} /\left(D^{1 \times p_{(i-1) i}} R_{i i}\right)$ depends only on $\operatorname{coker}_{D}\left(. R_{i i}\right)$ up to projective equivalence. Using Remark 1 and the exactness of the free resolution (24) of $M$, we find that the right $D$-modules

$$
\left\{\begin{array}{l}
\operatorname{coker}_{D}\left(. R_{i i}\right)=\operatorname{coker}_{D}\left(. R_{i}\right) \cong D^{1 \times p_{i-1}} R_{i-1}=\operatorname{ker}_{D}\left(. R_{i-2}\right), \quad i \geq 3, \\
\operatorname{coker}_{D}\left(. R_{22}\right)=\operatorname{coker}_{D}\left(. R_{2}\right)=D^{1 \times p_{1}} R_{1}=\operatorname{ker} \pi, \\
\operatorname{coker}_{D}\left(. R_{11}\right)=\operatorname{coker}_{D}\left(. R_{1}\right)=M,
\end{array}\right.
$$

depend on $M$ up to projective equivalence. Thus, the right $D$-module $N_{i i}$ depends only on $M$ up to a projective equivalence for $i \geq 1$. Using Remark $2, M_{i} \cong T_{i}=\operatorname{ext}_{D}^{i}\left(N_{i i}, D\right)$ finally depends only on $M$ for $i \geq 1$ and not on the free resolution 24) of $M$.

Let us state a few consequences of the above results.

Corollary 4. 1. The following long exact sequences of left D-modules hold

$$
0 \longrightarrow M_{i+1} \stackrel{\iota_{i+1}}{\longrightarrow} M_{i} \stackrel{\varepsilon_{i}}{\longrightarrow} \operatorname{ext}_{D}^{i}\left(\operatorname{ext}_{D}^{i}(M, D), D\right) \longrightarrow C_{i} \longrightarrow 0, \quad i=0, \ldots, n,
$$


where $C_{i}=\operatorname{coker} \varepsilon_{i}$ is isomorphic to a left D-submodule of $\operatorname{ext}_{D}^{i+2}\left(N_{(i+1)(i+1)}, D\right)$ for all $i=0, \ldots, n-2$ (with equality for $i=0$ ), $C_{n-1}=0, C_{n}=0$. In particular:

$$
M_{n} \cong \operatorname{ext}_{D}^{n}\left(\operatorname{ext}_{D}^{n}(M, D), D\right), \quad M_{n-1} / M_{n} \cong \operatorname{ext}_{D}^{n-1}\left(\operatorname{ext}_{D}^{n-1}(M, D), D\right) .
$$

2. If $M_{i}=0$, then $M_{i}=M_{i+1}=\ldots=M_{n}=0$.

3. $M=M_{j_{D}(M)}$.

Proof. 1. Using the last short exact sequence of (53), $M=M_{0}$ and $M_{1}=T_{1}$, we obtain (58) for $i=0$, where $C_{0}=\operatorname{ext}_{D}^{2}\left(N_{11}, D\right)$. Let us now suppose that $i=1, \ldots, n$ and let $\alpha_{i}=\gamma_{10} \circ \gamma_{21} \circ \gamma_{32} \circ \cdots \circ \gamma_{i(i-1)}$ be the left $D$-isomorphism from $T_{i}$ to $M_{i}$ (see (55)). Then, the long exact sequence (46) yields $[58)$ where $\iota_{i+1}=\alpha_{i} \circ \gamma_{(i+1) i} \circ \alpha_{i+1}^{-1}=\mathrm{id}_{M_{i+1}}, \varepsilon_{i}=\gamma_{i i} \circ \alpha_{i}^{-1}$ and $C_{i}=\operatorname{coker} \varepsilon_{i} \cong \operatorname{coker} \gamma_{i i} \subseteq \operatorname{ext}_{D}^{2+2}\left(N_{(i+1)(i+1)}, D\right)$ by 44 . Since $\operatorname{gld}(D)=n$, we get $C_{n-1}=C_{n}=0$. Finally, $(58)$ for $i=n, M_{n+1}=0$ and $C_{n}$ yield $M_{n} \cong \operatorname{ext}_{D}^{n}\left(\operatorname{ext}_{D}^{n}(M, D), D\right)$, and (58) for $i=n-1$ and $C_{n-1}=0$ implies that $M_{n-1} / M_{n} \cong \operatorname{ext}_{D}^{n-1}\left(\operatorname{ext}_{D}^{n-1}(M, D), D\right)$.

2. The equality is a direct consequence of (57).

3. If $j_{D}(M)=0$, then the result holds since $M=M_{0}$. Let us suppose that $j_{D}(M) \geq 1$. Then, $\operatorname{ext}_{D}^{i}\left(\operatorname{ext}_{D}^{i}(M, D), D\right)=0$ for $i=0, \ldots, j_{D}(M)-1$ since $\operatorname{ext}_{D}^{i}(M, D)=0$ for $i=0, \ldots, j_{D}(M)-1$. Using (58), we get $M_{i+1}=M_{i}$ for $i=1, \ldots, j_{D}(M)-1$. Finally, the last short exact sequence of (53) yields $M / M_{1}=0$, i.e., $M=M_{1}$, which finally proves the result.

Let us give consequences of the above results for an Auslander regular ring $D$.

Proposition 8. If $D$ is an Auslander regular ring and $\operatorname{gld}(D)=n$, then we have:

1. If $M_{i}$ is nonzero, then $j_{D}\left(M_{i}\right) \geq i$ for $i=0, \ldots, n$.

2. If $M_{i} / M_{i+1}$ is nonzero, then $M_{i} / M_{i+1}$ is an $i$-pure left $D$-module for $i=0, \ldots, n$. Moreover, if $M_{i+1}=0$, then $M_{i}$ is either zero or an $i$-pure left $D$-submodule of $M$. In particular, $M_{n}$ is either zero or a n-pure left D-module.

3. If $C_{i}$ is nonzero, then $j_{D}\left(C_{i}\right) \geq i+2$ for $i=0, \ldots, n-2$.

4. $M_{i}=M_{i+1}$ iff $\operatorname{ext}_{D}^{i}\left(\operatorname{ext}_{D}^{i}(M, D), D\right)=0$.

Proof. 1. Since $M_{i} \cong T_{i}=\operatorname{ext}_{D}^{i}\left(N_{i i}, D\right)$ for $i=1, \ldots, n$, Remark 7 then shows that $j_{D}\left(M_{i}\right) \geq i$. Moreover, $M_{0}=M$, and thus $j_{D}\left(M_{0}\right) \geq 0$.

2. By 3 of Theorem 8, $\operatorname{ext}_{D}^{i}\left(\operatorname{ext}_{D}^{i}(M, D), D\right)$ is either zero or $i$-pure, and so is the left $D$ module $M_{i} / M_{i+1} \cong \operatorname{im} \varepsilon_{i} \subseteq \operatorname{ext}_{D}^{i}\left(\operatorname{ext}_{D}^{i}(M, D), D\right)$ (see Remark 6). In particular, if $M_{i+1}=0$, then $M_{i}$ is either zero or an $i$-pure left $D$-submodule of $M$. Finally, $M_{n} \cong \operatorname{ext}_{D}^{n}\left(\operatorname{ext}_{D}^{n}(M, D), D\right)$ (see 1 of Corollary 4 implies that $M_{n}$ is either zero or $n$-pure.

3. Since $C_{i}=\operatorname{coker} \varepsilon_{i}$ is isomorphic to a left $D$-submodule of $\operatorname{ext}_{D}^{i+2}\left(N_{(i+1)(i+1)}, D\right)$ for $i=0, \ldots, n-2$ (see 1 of Corollary 4 ), then Remark 7 then yields $j_{D}\left(C_{i}\right) \geq i+2$ for $i=0, \ldots, n-2$.

4. If $M_{i}=M_{i+1}$, then (58) gives $C_{i} \cong \operatorname{ext}_{D}^{i}\left(\operatorname{ext}_{D}^{i}(M, D), D\right)$. On the one hand, by 3 of Theorem 8, $C_{i}$ is either zero or $i$-pure, and thus we either have $C_{i}=0$ or $j_{D}\left(C_{i}\right)=i$. On the other hand, using 3 , if $C_{i} \neq 0$, then $j_{D}\left(C_{i}\right) \geq i+2$, which shows that $C_{i}=0$. Conversely, if $\operatorname{ext}_{D}^{i}\left(\operatorname{ext}_{D}^{i}(M, D), D\right)=0$, then (58) yields $M_{i}=M_{i+1}$. 
If $D$ is also a Cohen-Macaulay ring, then using Corollary 3, we obtain:

$$
\forall i=0, \ldots, n, \quad \operatorname{dim}_{D}\left(M_{i}\right) \leq \operatorname{dim}(D)-i, \quad \operatorname{dim}_{D}\left(M_{i} / M_{i+1}\right)=\operatorname{dim}(D)-i .
$$

Let us now show that the filtration $\left\{M_{i}\right\}_{i=0, \ldots, n}$ of $M$ defined by (55) is exactly the grade filtration $\left\{t_{i}(M)\right\}_{i=0, \ldots, n}$ of $M$ defined in (21) when $D$ is an Auslander regular ring.

Theorem 10. Let $D$ be an Auslander regular ring and $M$ a finitely generated left $D$-module. Then, we have $t_{i}(M)=M_{i}$ for all $i=0, \ldots, n=\operatorname{gld}(D)$, i.e., the grade filtration (22) of $M$ and the filtration (7) of $M$ coincide.

Proof. Let us first prove that $0 \neq M_{i} \subseteq t_{i}(M)$. By 1 of Proposition 8, $j_{D}\left(M_{i}\right) \geq i$. If $m \in M_{i}$, then applying Proposition 7 to the short exact sequence $0 \longrightarrow D m \longrightarrow M_{i} \longrightarrow M_{i} /(D m) \longrightarrow 0$, we obtain $j_{D}(D m) \geq j_{D}\left(M_{i}\right)=i$, and thus $m \in t_{i}(M)$, i.e., $M_{i} \subseteq t_{i}(M)$.

Following [9], let us now prove $t_{i}(M) \subseteq M_{i}$ by induction on $i$, i.e., $t_{i}(M)=M_{i}$ by the above point. We first note that $t_{0}(M)=M=M_{0}$, which proves the result for $i=0$. Let us now assume that $t_{i}(M)=M_{i}$ and let us show that it yields $t_{i+1}(M)=M_{i+1}$. Since $M_{i+1} \subseteq t_{i+1}(M) \subseteq t_{i}(M)$, we get $t_{i+1}(M) / M_{i+1} \subseteq t_{i}(M) / M_{i+1}=M_{i} / M_{i+1}$. Using 2 of Proposition 8, $M_{i} / M_{i+1}$ is either zero or an $i$-pure left $D$-module. If $M_{i} / M_{i+1}=0$, then $t_{i+1}(M) / M_{i+1}=0$, i.e., $t_{i+1}(M)=M_{i+1}$, which proves the result. Hence, let us assume that $M_{i} / M_{i+1}$ is an $i$-pure left $D$-module. Then, by definition of a pure module, its left $D$-submodule $t_{i+1}(M) / M_{i+1}$ is also either zero or $i$-pure. If $t_{i+1}(M) / M_{i+1}$ is $i$-pure, then $j_{D}\left(t_{i+1}(M) / M_{i+1}\right)=i$. But, applying Proposition 7 to the following short exact sequence

$$
0 \longrightarrow M_{i+1} \longrightarrow t_{i+1}(M) \longrightarrow t_{i+1}(M) / M_{i+1} \longrightarrow 0
$$

gives $j_{D}\left(t_{i+1}(M) / M_{i+1}\right) \geq j_{D}\left(t_{i+1}(M)\right) \geq i+1$, which yields a contradiction. Thus, we obtain $t_{i+1}(M) / M_{i+1}=0$, i.e., $t_{i+1}(M)=M_{i+1}$, which finally proves the result by induction.

Remark 11. We can combine Theorem 10 and Proposition 8 to find again 2 of Theorem 8 . Indeed, using Theorem 10, $M \neq 0$ is $i$-pure iff $M=M_{1}=\ldots=M_{i} \neq 0$ and $M_{i+1}=M_{i+2}=$ $\ldots=M_{n+1}=0$. By 4 of Proposition 8, the equalities are equivalent to $\operatorname{ext}_{D}^{k}\left(\operatorname{ext}_{D}^{k}(M, D), D\right)=0$ for $k=0, \ldots, i-1$ and $k=i+1, \ldots, n$. Let us study the inequality. Combining $M_{i} \neq 0, M_{i+1}=0$ and (58), $\operatorname{ext}_{D}^{i}\left(\operatorname{ext}_{D}^{i}(M, D), D\right)$ then contains the nonzero left $D$-submodule $M_{i}$, which shows that $\operatorname{ext}_{D}^{i}\left(\operatorname{ext}_{D}^{i}(M, D), D\right) \neq 0$. Since $\operatorname{ext}_{D}^{i}\left(\operatorname{ext}_{D}^{i}(M, D), D\right) \neq 0$ yields $M \neq 0, M \neq 0$ is then an $i$-pure left $D$-module iff $\operatorname{ext}_{D}^{k}\left(\operatorname{ext}_{D}^{k}(M, D), D\right)=0$ for $k \neq i$ and $\operatorname{ext}_{D}^{i}\left(\operatorname{ext}_{D}^{i}(M, D), D\right) \neq 0$.

The existence of the filtration (57) only requires that $D$ is a noetherian regular domain which satisfies (38). If $D$ is an Auslander regular ring, then Theorem 10 proves that (57) is exactly the grade filtration (22) of $M$. If $D$ is also a Cohen-Macaulay ring, then using (59), the filtration $\left\{M_{i}\right\}_{i=0, \ldots, n}$ of $M$ gives a built-in classification of the elements of $M$ by means of their (co)dimensions. This filtration is sometimes called the codimension filtration of $M$ (or equidimensional decomposition in algebraic geometry).

Remark 12. If $D$ satisfies the hypotheses of Theorem 9, then Theorem 9 shows that the characteristic ideal $J(M)$ of $\operatorname{gr}(D)$ does not depend on the choice of a good filtration of $M$. The characteristic variety of $M$ is then defined by $\operatorname{char}(M)=\{\mathfrak{p} \in \operatorname{Spec}(\operatorname{gr}(D)) \mid J(M) \subseteq \mathfrak{p}\}$, where $\operatorname{Spec}(\operatorname{gr}(D))$ is the set of prime ideals of $\operatorname{gr}(D)$ endowed with the Zariski topology. A well-known result in algebraic analysis states that a short exact sequence of left $D$-modules

$$
0 \longrightarrow M^{\prime} \longrightarrow M \longrightarrow M^{\prime \prime} \longrightarrow 0
$$


yields the equality $\operatorname{char}(M)=\operatorname{char}\left(M^{\prime}\right) \cup \operatorname{char}\left(M^{\prime \prime}\right)$ (see, e.g., [30, 33]). Applying this result to the short exact sequences $0 \longrightarrow M_{i+1} \longrightarrow M_{i} \longrightarrow M_{i} / M_{i+1} \longrightarrow 0$ for $i=0, \ldots, n$, we get:

$$
\operatorname{char}(M)=\bigcup_{i=0, \ldots, n} \operatorname{char}\left(M_{i} / M_{i+1}\right)
$$

It can be proved that the characteristic variety $\operatorname{char}(P)$ of an $i$-pure module $P$ is equidimensional in the sense that every irreducible component of $\operatorname{char}(P)$ has dimension $\operatorname{dim}(D)-i$ (see, e.g., [10]). Hence, 60 is an equidimensional decomposition of the affine algebraic variety char $(M)$.

Theorem 10 shows that the grade filtration of $M$ can be computed by means of elementary methods of module theory and homological algebra. In particular, we do not need to compute a Cartan-Eilenberg resolution $P^{\bullet \bullet}$ (see, e.g., [50]) of the complex 25) (called $\operatorname{Rhom}(M, D)$ within derived categories (see, e.g., [25])), the total complex $\operatorname{Tot}\left(\operatorname{hom}_{D}\left(P^{\bullet \bullet}, D\right)\right)$ of the double complex $\operatorname{hom}_{D}\left(P^{\bullet \bullet}, D\right)$, and the spectral sequence associated with the first filtration of $\operatorname{Tot}\left(\operatorname{hom}_{D}\left(P^{\bullet \bullet}, D\right)\right)$. For more details, see [2, 9, 10, 11, 23, 25, 32, 50]. Our approach has then the advantage to be easily implementable in any computer algebra system containing an implementation of Gröbner bases for (noncommutative) polynomial rings (e.g., Maple, Singular, Macaulay2, Magma, Mathematica). Another advantage will be explained in Section 4.

The filtration (57) is a particular case of the more general bidualizing filtration $\left\{M_{i}\right\}_{i=0, \ldots, n}$ of a finitely generated module $M$ over a regular ring $D$ [9, 10], of which the existence can be proved by means of a spectral sequence argument. In this case, $M_{i} / M_{i+1}$ is then a left $D$ -

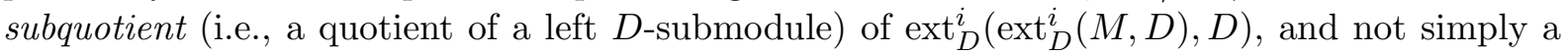
left $D$-submodule as shown above for an Auslander regular ring $D$. Finally, we note that the results developed in [9, 49] were extended in [32] for an Auslander-Gorenstein ring D, namely a noetherian ring of finite injective dimension $m$ as a left/right $D$-module (i.e., $\operatorname{ext}_{D}^{i}(M, D)=0$ for $i>m$ and for all finitely left/right $D$-modules $M$ ) [50] which satisfies the Auslander condition (see Definition 8).

Let us sum up the above results in the following algorithm.

Algorithm 1. - Input: A noetherian regular $\operatorname{ring} D$ satisfying (38), $\operatorname{gld}(D)=n$, and $R \in D^{q \times p}$.

- Output: A sequence $\left\{T_{i}\right\}_{i=1, \ldots, n}$ of finitely generated left $D$-modules defined by 45 and a sequence $\left\{\gamma_{10} \in \operatorname{hom}_{D}\left(T_{1}, M\right)\right\} \cup\left\{\gamma_{(i+1) i} \in \operatorname{hom}_{D}\left(T_{i+1}, T_{i}\right)\right\}_{i=1, \ldots, n}$ of $D$-homomorphisms defined by 49 and $(48)$ such that $\left\{M_{i}=\left(\gamma_{10} \circ \gamma_{21} \circ \gamma_{32} \circ \cdots \circ \gamma_{i(i-1)}\right)\left(T_{i}\right)\right\}_{i=1, \ldots, n}$ is a filtration of $M$ (the grade filtration of $M$ when $D$ is an Auslander regular ring).

1. Set $R_{1}=R, p_{1}=p, p_{2}=q$, and $M=D^{1 \times p_{1}} /\left(D^{1 \times p_{2}} R_{1}\right)$.

2. Compute matrices $R_{k} \in D^{p_{k} \times p_{k-1}}$ for $k=2, \ldots, n$ such that 24 is an exact sequence.

3. Set $p_{k k}=p_{k}, p_{(k-1) k}=p_{k-1}=p_{(k-1)(k-1)}, R_{k k}=R_{k}$, and $N_{k k}=D^{p_{k k}} /\left(R_{k k} D^{p_{(k-1) k}}\right)$.

4. For $k=1, \ldots, n$ and for $j=1, \ldots, k$, compute matrices $R_{(k-j) k} \in D^{p_{(k-j) k} \times p_{(k-j-1) k}}$ such that (27) is an exact sequence.

5. For $k=2, \ldots, n$, compute matrices $F_{(k-2) k} \in D^{p_{(k-2) k} \times p_{(k-2)(k-1)}}$ such that:

$$
R_{(k-1)(k-1)}=R_{(k-1) k} F_{(k-2) k} .
$$


6. For $k=2, \ldots, n$ and for $j=2, \ldots, k$, compute $F_{(k-j) k} \in D^{p_{(k-j) k} \times p_{(k-j)(k-1)}}$ satisfying:

$$
F_{(k-j) k} R_{(k-j)(k-1)}=R_{(k-j) k} F_{(k-j-1) k} .
$$

7. Return the matrices $R_{0 i}, R_{1 i}$, and $F_{0 i}$ defining the left $D$-module $T_{i}=\operatorname{ker}_{D}\left(. R_{0 i}\right) / \operatorname{im}_{D}\left(. R_{1 i}\right)$ for $i=1, \ldots, n, \gamma_{10}=\mathrm{id}_{T_{1}}: T_{1}=\operatorname{ker}_{D}\left(. R_{01}\right) / \operatorname{im}_{D}\left(. R_{11}\right) \longrightarrow M=D^{1 \times p_{01}} / \operatorname{im}_{D}\left(. R_{11}\right)$, and $\gamma_{i(i-1)} \in \operatorname{hom}_{D}\left(T_{i}, T_{i-1}\right)$ by 48$)$ for $i=2, \ldots, n$.

Remark 13. Using 3 of Corollary 4 i.e., $M=M_{j_{D}(M)}$, let us explain how Algorithm 1 can then be speeded up when $j_{D}(M) \geq 1$ by avoiding the computation of the left $D$-modules $T_{i}$ 's for $i=1, \ldots, j_{D}(M)$. Since $\operatorname{ext}_{D}^{i}(M, D)=0$ for $i=0, \ldots, j_{D}(M)-1$, then 25 yields the following free resolution of $N_{j_{D}(M) j_{D}(M)}$ :

$$
D^{p_{0}} \stackrel{R_{1} \cdot}{\longrightarrow} D^{p_{1}} \stackrel{R_{2} .}{\longrightarrow} \ldots \stackrel{R_{j_{D}(M)}}{\longrightarrow} D^{p_{j_{D}}(M)} \stackrel{\kappa_{j_{D}(M) j_{D}(M)}}{\longrightarrow} N_{j_{D}(M) j_{D}(M)} \longrightarrow 0 .
$$

Applying Proposition 1 to 61 , we get $\operatorname{ext}_{D}^{j_{D}(M)}\left(N_{j_{D}(M) j_{D}(M)}, D\right) \cong \operatorname{ext}_{D}^{1}\left(N_{11}, D\right)=M_{1}$, where $N_{11}=D^{p_{1}} /\left(R_{1} D^{p_{0}}\right)$. Moreover, since $j_{D}(M) \geq 1$, $\operatorname{hom}_{D}(M, D)=0$, and using Theorem 1. $\operatorname{ker}_{D}\left(R_{1}.\right) \cong \operatorname{hom}_{D}(M, D)=0$, and thus $M_{1}=\operatorname{ext}_{D}^{1}\left(N_{11}, D\right) \cong M$. Hence, we do not need to compute the beginning of a free resolution of the right $D$-module $N_{k k}$ for $k=1, \ldots, j_{D}(M)$, i.e., we can only consider $k=j_{D}(M)+1, \ldots, n$ in 4 of Algorithm 1 .

Algorithm 1 with its improvement explained in Remark 13 are implemented in the Maple package PurityFiltration [45] built upon Oremodules [15]. The PurityFiltration package allows us to compute the grade filtration of a finitely generated left $D$-module $M$, where $D$ is an Ore algebras available in OreModules. If an involution $\theta$ of $D$ (namely, $\theta: D \longrightarrow D$ satisfies $\theta\left(d_{1}+d_{2}\right)=\theta\left(d_{1}\right)+\theta\left(d_{2}\right), \theta\left(d_{1} d_{2}\right)=\theta\left(d_{2}\right) \theta\left(d_{1}\right)$ for all $d_{1}, d_{2} \in D$, and $\left.\theta^{2}=\operatorname{id}_{D}\right)$ exists, then we can compute the matrices $R_{(k-j) k}$ defined in 4 of Algorithm 1 by left Gröbner basis techniques. For more details, see [14]. Algorithm 1 has also recently been implemented in the homalg based package AbelianSystems [7] by M. Barakat (University of Kaiserslautern) and the author.

Let us now determine a finite presentation of the left $D$-modules $T_{i}$ 's defined by (45). To do that, we first consider the beginning of a finite free resolution of $P_{i}=D^{1 \times p_{-1 i}} /\left(D^{1 \times p_{0 i}} R_{0 i}\right)$, namely, matrices $R_{1 i}^{\prime} \in D^{p_{1 i}^{\prime} \times p_{0 i}}$ and $R_{2 i}^{\prime} \in D^{p_{2 i}^{\prime} \times p_{1 i}^{\prime}}$ such that $\operatorname{ker}_{D}\left(. R_{0 i}\right)=D^{1 \times p_{1 i}^{\prime}} R_{1 i}^{\prime}$ and $\operatorname{ker}_{D}\left(. R_{1 i}^{\prime}\right)=D^{1 \times p_{2 i}^{\prime} R_{2 i}^{\prime}}$ for $i=1, \ldots, n$. We obtain the commutative diagram 68 formed by horizontal exact sequences.

Remark 14. If $R_{0 k}=0$, i.e., $\operatorname{ker}_{D}\left(R_{1 k}.\right)=0$, then applying the functor $\operatorname{hom}_{D}(\cdot, D)$ to the short exact sequence $0 \longrightarrow D^{p_{0 k}} \stackrel{R_{1 k}}{\longrightarrow} D^{p_{1 k}} \stackrel{\kappa_{1 k}}{\longrightarrow} N_{1 k} \longrightarrow 0$, we get the following complex:

$$
0 \longleftarrow D^{1 \times p_{0 k}} \stackrel{R_{1 k}}{\longleftarrow} D^{1 \times p_{1 k}} .
$$

Hence, we have $\operatorname{ker}_{D}\left(. R_{0 k}\right)=D^{1 \times p_{0 k}}$, i.e., $R_{1 k}^{\prime}=I_{p_{0 k}}, p_{1 k}^{\prime}=p_{0 k}$, and $R_{2 k}^{\prime}=0$.

Combining 56 with $\operatorname{ker}_{D}\left(. R_{0 i}\right)=D^{1 \times p_{1 i}^{\prime}} R_{1 i}^{\prime}$, we obtain the following explicit characterization of the $M_{i}$ 's, i.e., of the $t_{i}(M)$ 's when $D$ is an Auslander regular ring (see Theorem 10):

$$
\left\{\begin{array}{l}
M_{1}=\left(D^{1 \times p_{11}^{\prime}} R_{11}^{\prime}\right) /\left(D^{1 \times p_{11}} R_{11}\right), \\
M_{i}=\left(D^{1 \times p_{1 i}^{\prime}}\left(R_{1 i}^{\prime} F_{0 i} \ldots F_{02}\right)\right) /\left(D^{1 \times p_{11}} R_{11}\right), \quad i=2, \ldots, n .
\end{array}\right.
$$

Hence, (62) shows that the residue classes of the rows of the matrix $R_{1 i}^{\prime} F_{0 i} \ldots F_{02}$ in the left $D$-module $M=D^{1 \times p_{01}} /\left(D^{1 \times p_{11}} R_{11}\right)$ generate the left $D$-module $M_{i}$. 
Algorithm 2. - Input: A noetherian regular ring $D$ satisfying $(38), \operatorname{gld}(D)=n$, and $R \in D^{q \times p}$.

- Output: A sequence $\left\{M_{i}\right\}_{i=1, \ldots, n}$ of left $D$-submodules of $M$ defined by (62), i.e., the grade filtration (57) of $M$ when $D$ is an Auslander regular ring.

1. Apply Algorithm 1 to $D$ and $R \in D^{q \times p}$ to obtain $R_{0 i} \in D^{p_{0 i} \times p_{-1 i}}$ for $i=1, \ldots, n$, and $F_{0 i} \in D^{p_{0 i} \times p_{0(i-1)}}$ for $i=2, \ldots, n$.

2. Compute $R_{1 i}^{\prime} \in D^{p_{1 i}^{\prime} \times p_{0 i}}$ such that $\operatorname{ker}_{D}\left(. R_{0 i}\right)=D^{1 \times p_{1 i}^{\prime}} R_{1 i}^{\prime}$ for $i=1, \ldots, n$.

3. Return the matrices $R_{1 i}^{\prime} F_{0 i} \ldots F_{02}$ (or their reductions with respect to $D^{1 \times p_{11}} R_{11}$ ) for $i=1, \ldots, n$.

Algorithm 2 is implemented in the PurityFiltration package [45].

Let us now compute a finite presentation of the left $D$-module $M_{i}$ 's. The identity $R_{1 i} R_{0 i}=0$ yields $D^{1 \times p_{1 i}} R_{1 i} \subseteq \operatorname{ker}_{D}\left(. R_{0 i}\right)=D^{1 \times p_{1 i}^{\prime}} R_{1 i}^{\prime}$, and thus there exists $R_{1 i}^{\prime \prime} \in D^{p_{1 i} \times p_{1 i}^{\prime}}$ such that:

$$
\forall i=1, \ldots, n, \quad R_{1 i}=R_{1 i}^{\prime \prime} R_{1 i}^{\prime} .
$$

Applying Proposition 4 to the left $D$-module $T_{i}$, we obtain

$$
\begin{aligned}
\forall i=1, \ldots, n, \quad T_{i} & =\operatorname{ker}_{D}\left(. R_{0 i}\right) / \operatorname{im}_{D}\left(. R_{1 i}\right)=\left(D^{1 \times p_{1 i}^{\prime}} R_{1 i}^{\prime}\right) /\left(D^{1 \times p_{1 i}} R_{1 i}\right) \\
& \cong L_{i} \triangleq D^{1 \times p_{1 i}^{\prime}} /\left(D^{1 \times p_{1 i}} R_{1 i}^{\prime \prime}+D^{1 \times p_{2 i}^{\prime}} R_{2 i}^{\prime}\right),
\end{aligned}
$$

where the above left $D$-isomorphism $\chi_{i}$ is defined by

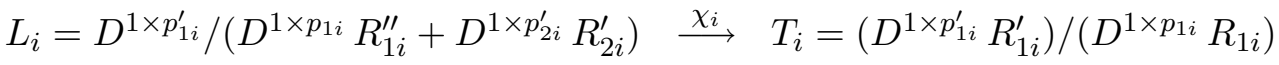

$$
\begin{aligned}
& \rho_{i}^{\prime}(\lambda) \longmapsto \rho_{i}\left(\lambda R_{1 i}^{\prime}\right),
\end{aligned}
$$

and $\rho_{i}^{\prime}: D^{1 \times p_{1 i}^{\prime}} \longrightarrow L_{i}$ is the canonical projection onto the left $D$-module $L_{i}$. The inverse $\chi_{i}^{-1} \in \operatorname{hom}_{D}\left(T_{i}, L_{i}\right)$ is then defined by $\chi_{i}^{-1}\left(\rho_{i}\left(\lambda R_{1 i}^{\prime}\right)\right)=\rho_{i}^{\prime}(\lambda)$ for all $\lambda \in D^{1 \times p_{1 i}^{\prime}}$.

Let us complete the commutative diagram $(68)$ to determine the left $D$-homomorphism $\bar{\gamma}_{(i+1) i}$ induced by the left $D$-homomorphism $\gamma_{(i+1) i}$ and the left $D$-isomorphisms $\chi_{i}$ and $\chi_{i+1}$. Using (30) with $k=j=i$ and $i=2, \ldots, n$, we obtain $F_{0 i} R_{0(i-1)}=R_{0 i} F_{-1 i}$. Pre-multiplying this identity by $R_{1 i}^{\prime}$, we get $R_{1 i}^{\prime} F_{0 i} R_{0(i-1)}=R_{1 i}^{\prime} R_{0 i} F_{-1 i}=0$, and thus $D^{1 \times p_{1 i}^{\prime}}\left(R_{1 i}^{\prime} F_{0 i}\right) \subseteq$ $\operatorname{ker}_{D}\left(. R_{0(i-1)}\right)=D^{1 \times p_{1(i-1)}^{\prime}} R_{1(i-1)}^{\prime}$, which proves the existence of $F_{1 i}^{\prime} \in D^{p_{1 i}^{\prime} \times p_{1(i-1)}^{\prime}}$ such that:

$$
\forall i=2, \ldots, n, \quad R_{1 i}^{\prime} F_{0 i}=F_{1 i}^{\prime} R_{1(i-1)}^{\prime} .
$$

Similarly, we can prove the existence of a matrix $F_{2 i}^{\prime} \in D^{p_{2 i}^{\prime} \times p_{2(i-1)}^{\prime}}$ such that:

$$
\forall i=2, \ldots, n, \quad R_{2 i}^{\prime} F_{1 i}^{\prime}=F_{2 i}^{\prime} R_{2(i-1)}^{\prime} .
$$

Thus, the commutative diagram $(69)$ formed by horizontal exact sequences holds.

Let us now deduce identities which will be used in what follows. Combining (28), (29), (30), (63) and (66), for $i=1, \ldots, n$, we get

$$
\begin{aligned}
F_{1(i+1)}\left(R_{1 i}^{\prime \prime} R_{1 i}^{\prime}\right)=F_{1(i+1)} R_{1 i}=R_{1(i+1)} F_{0(i+1)} & =\left(R_{1(i+1)}^{\prime \prime} R_{1(i+1)}^{\prime}\right) F_{0(i+1)} \\
& =R_{1(i+1)}^{\prime \prime} F_{1(i+1)}^{\prime} R_{1 i}^{\prime},
\end{aligned}
$$



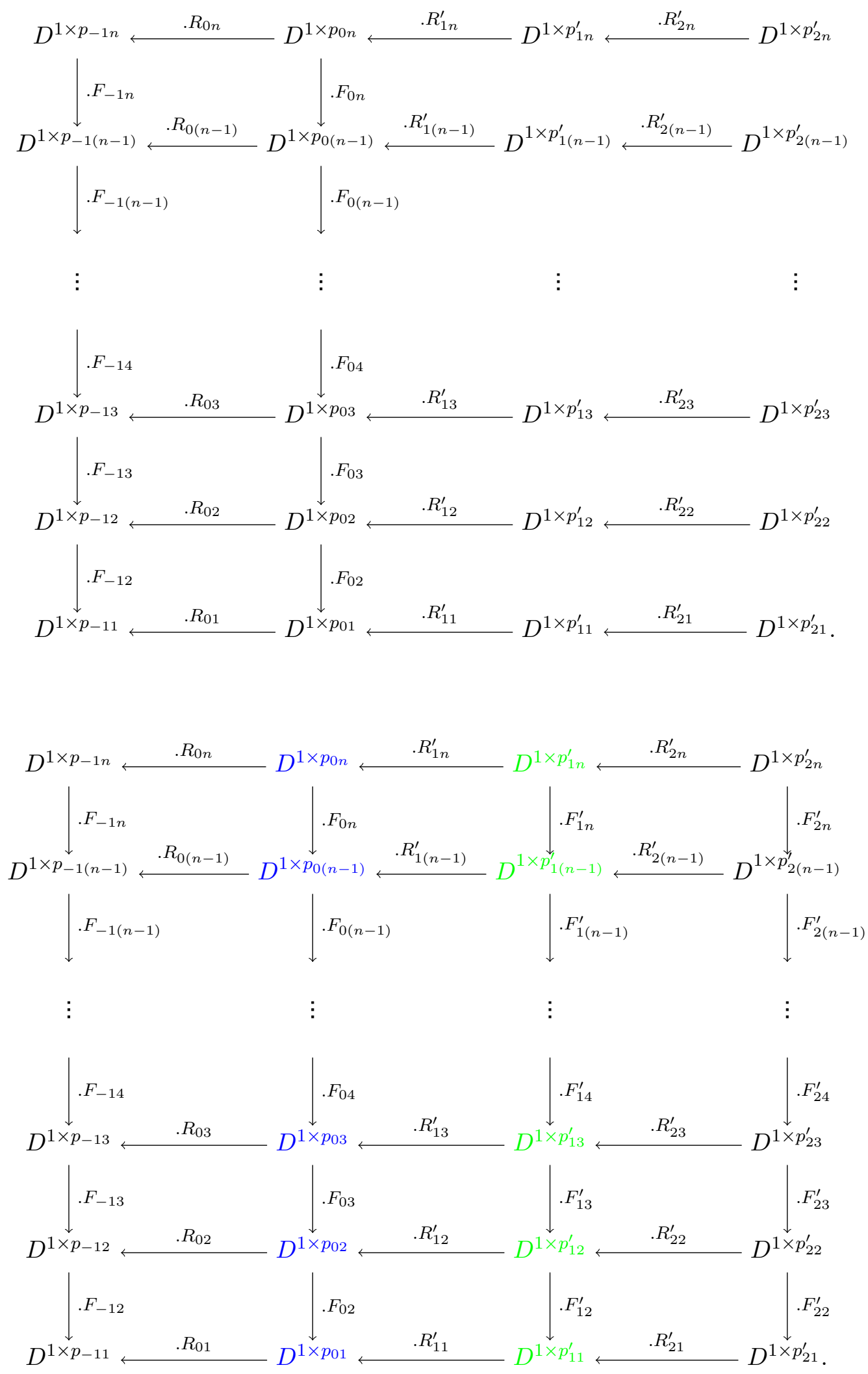


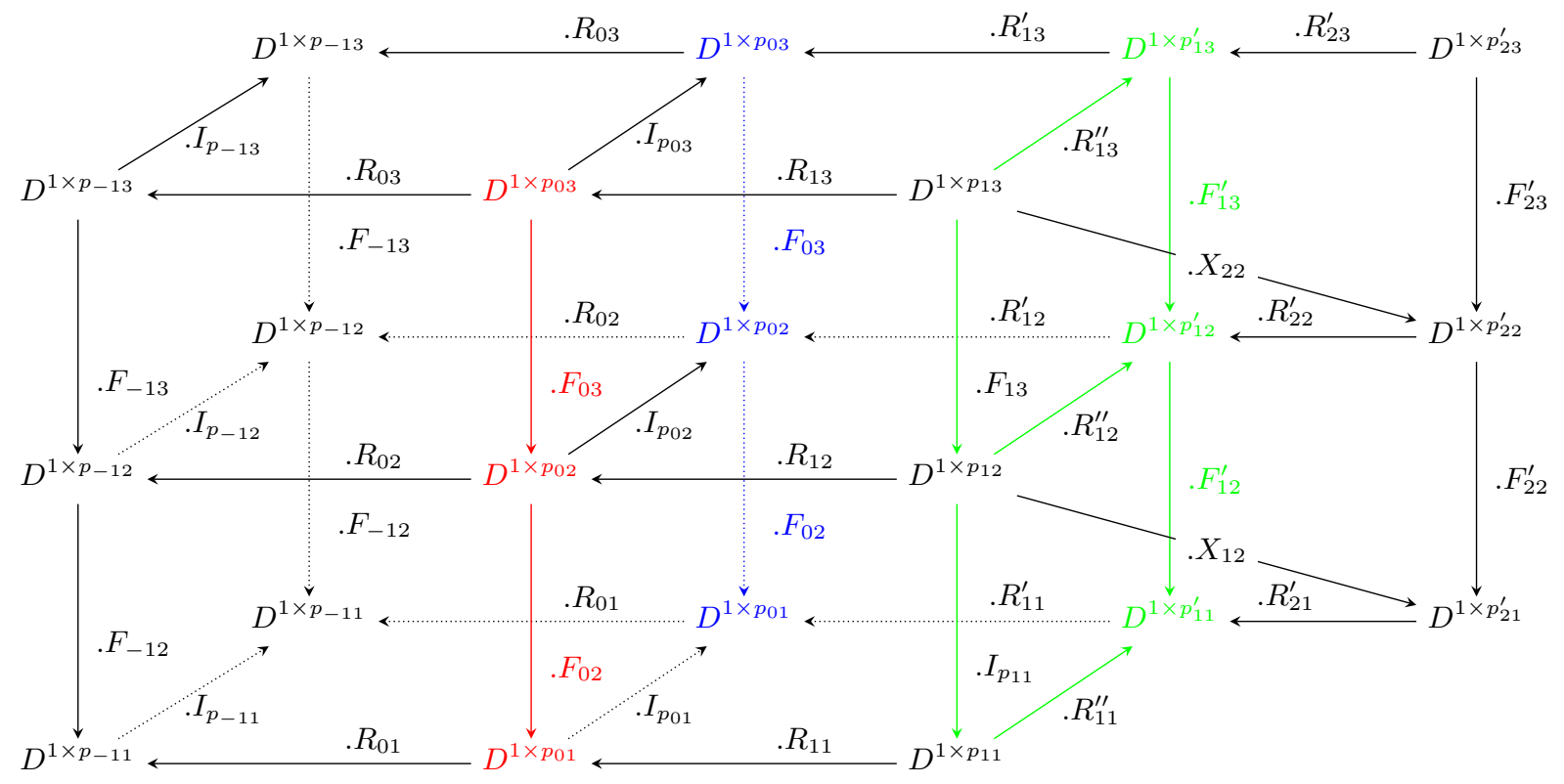

Figure 1: Bottom part of the main diagram defining the grade filtration of $M$

and thus $\left(F_{1(i+1)} R_{1 i}^{\prime \prime}-R_{1(i+1)}^{\prime \prime} F_{1(i+1)}^{\prime}\right) R_{1 i}^{\prime}=0$, i.e.,

$$
D^{1 \times p_{1(i+1)}}\left(F_{1(i+1)} R_{1 i}^{\prime \prime}-R_{1(i+1)}^{\prime \prime} F_{1(i+1)}^{\prime}\right) \subseteq \operatorname{ker}_{D}\left(. R_{1 i}^{\prime}\right)=D^{1 \times p_{2 i}^{\prime}} R_{2 i}^{\prime},
$$

which proves the existence of a matrix $X_{i 2} \in D^{p_{1(i+1)} \times p_{2 i}^{\prime}}$ such that:

$$
\forall i=1, \ldots, n-1, \quad F_{1(i+1)} R_{1 i}^{\prime \prime}-R_{1(i+1)}^{\prime \prime} F_{1(i+1)}^{\prime}=X_{i 2} R_{2 i}^{\prime} .
$$

Now, $\gamma_{(i+1) i} \in \operatorname{hom}_{D}\left(T_{i+1}, T_{i}\right)$ then gives rise to $\bar{\gamma}_{(i+1) i} \in \operatorname{hom}_{D}\left(L_{i+1}, L_{i}\right)$ defined by

$$
\forall i=1, \ldots, n-1, \quad \bar{\gamma}_{(i+1) i}=\chi_{i}^{-1} \circ \gamma_{(i+1) i} \circ \chi_{i+1},
$$

where the $\chi_{i}$ 's are defined by $(65)$ and $\gamma_{(i+1) i}$ is defined by (48). Using (66), we get

$$
\begin{aligned}
\bar{\gamma}_{(i+1) i}\left(\rho_{(i+1)}^{\prime}(\lambda)\right) & =\left(\chi_{i}^{-1} \circ \gamma_{(i+1) i}\right)\left(\rho_{i+1}\left(\lambda R_{1(i+1)}^{\prime}\right)\right)=\chi_{i}^{-1}\left(\rho_{i}\left(\lambda R_{1(i+1)}^{\prime} F_{0(i+1)}\right)\right) \\
& =\chi_{i}^{-1}\left(\rho_{i}\left(\lambda F_{1(i+1)}^{\prime} R_{1 i}^{\prime}\right)\right)=\rho_{i}^{\prime}\left(\lambda F_{1(i+1)}^{\prime}\right),
\end{aligned}
$$

for all $\lambda \in D^{1 \times p_{1(i+1)}^{\prime}}$. Moreover, using (67) and 70 , for $i=1, \ldots, n-1$, we obtain

$$
\left(\begin{array}{l}
R_{1(i+1)}^{\prime \prime} \\
R_{2(i+1)}^{\prime}
\end{array}\right) F_{1(i+1)}^{\prime}=\left(\begin{array}{c}
F_{1(i+1)} R_{1 i}^{\prime \prime}-X_{i 2} R_{2 i}^{\prime} \\
F_{2(i+1)}^{\prime} R_{2 i}^{\prime}
\end{array}\right)=\left(\begin{array}{cc}
F_{1(i+1)} & -X_{i 2} \\
0 & F_{2(i+1)}^{\prime}
\end{array}\right)\left(\begin{array}{c}
R_{1 i}^{\prime \prime} \\
R_{2 i}^{\prime}
\end{array}\right)
$$

which yields the following commutative exact diagram

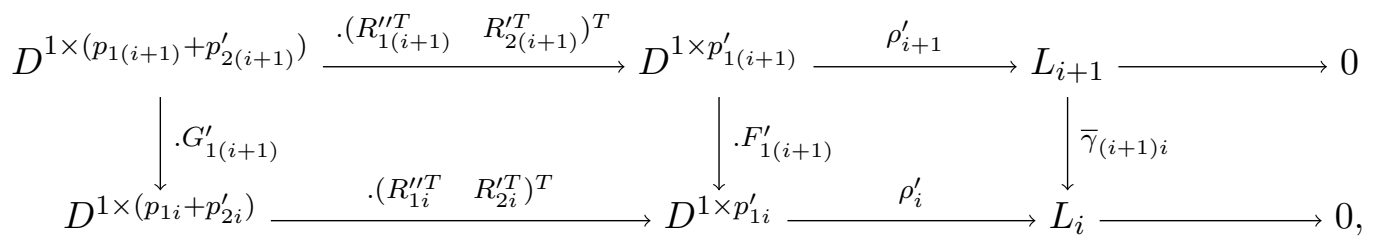


where $G_{1(i+1)}^{\prime} \in D^{\left(p_{1(i+1)}+p_{2(i+1)}^{\prime}\right) \times\left(p_{1 i}+p_{2 i}^{\prime}\right)}$ is the first matrix appearing in the last equality of 73.).

The identities $R_{11}=R_{11}^{\prime \prime} R_{11}^{\prime}$ (see $(63)$ ) and $R_{21}^{\prime} R_{11}^{\prime}=0$ yield the following commutative exact diagram

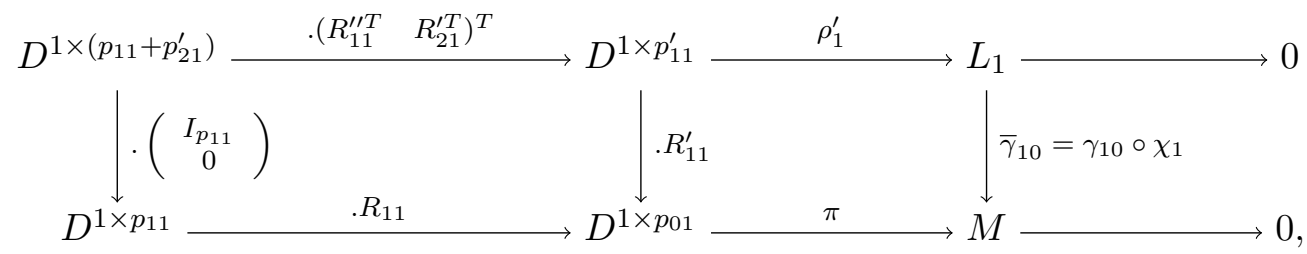

where $\bar{\gamma}_{10}=\gamma_{10} \circ \chi_{1} \in \operatorname{hom}_{D}\left(L_{1}, M\right)$ is defined by:

$$
\forall \lambda \in D^{1 \times p_{11}^{\prime}}, \quad \bar{\gamma}_{10}\left(\rho_{1}^{\prime}(\lambda)\right)=\pi\left(\lambda R_{11}^{\prime}\right) .
$$

The matrices previously introduced can be rearranged into the three dimensional diagram whose bottom part is shown in Figure 1. Each two dimensional diagram of Figure 1 commutes except for the two diagrams marked in green ("faces in the depth direction") (see 700 ). The horizontal sequences are either complexes (marked in red) or are exact sequences (marked in blue and in green). The vertical sequences are not complexes. The defect of exactness $T_{i}=\operatorname{ext}_{D}^{i}\left(N_{i i}, D\right)$ of the $i^{\text {th }}$ horizontal complex at $D^{1 \times p_{0 i}}$ (marked in red) is isomorphic to the cokernel $L_{i}$ of the left $D$-homomorphism $D^{1 \times\left(p_{1 i}+p_{2 i}^{\prime}\right)} \longrightarrow D^{1 \times p_{1 i}^{\prime}}$ defined by the two left $D$-homomorphisms . $R_{1 i}^{\prime \prime}: D^{1 \times p_{1 i}} \longrightarrow D^{1 \times p_{1 i}^{\prime}}$ and $\cdot R_{2 i}^{\prime}: D^{1 \times p_{2 i}^{\prime}} \longrightarrow D^{1 \times p_{1 i}^{\prime}}$ arriving at $D^{1 \times p_{1 i}^{\prime}}$ (marked in green),

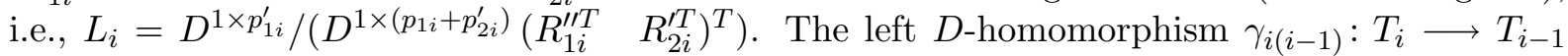
defined by (48), i.e., by means of the left $D$-homomorphism.$F_{0 i}$ (marked in red), induces $\bar{\gamma}_{i(i-1)} \in \operatorname{hom}_{D}\left(L_{i}, L_{i-1}\right)$ defined by $(72)$, i.e., by means of the left $D$-homomorphism.$F_{1 i}^{\prime}$ (marked in green).

Algorithm 3. - Input: A noetherian regular ring $D$ satisfying $(38), \operatorname{gld}(D)=n$, and $R \in D^{q \times p}$.

- Output: A sequence $\left\{L_{i}\right\}_{i=1, \ldots, n}$ of finitely presented left $D$-modules and a sequence $\left\{\bar{\gamma}_{10} \in \operatorname{hom}_{D}\left(L_{1}, M\right)\right\} \cup\left\{\bar{\gamma}_{(i+1) i} \in \operatorname{hom}_{D}\left(L_{i+1}, L_{i}\right)\right\}_{i=1, \ldots, n-1}$ of left $D$-homomorphisms defined by 65 .

1. Apply Algorithm 2 to $D$ and $R \in D^{q \times p}$ to get matrices $R_{0 i} \in D^{p_{0 i} \times p_{-1 i}}$ for $i=1, \ldots, n$, matrices $F_{0 i} \in D^{p_{0 i} \times p_{0(i-1)}}$ for $i=2, \ldots, n$, and matrices $R_{1 i}^{\prime} \in D^{p_{1 i}^{\prime} \times p_{0 i}}$ such that $\operatorname{ker}_{D}\left(. R_{0 i}\right)=D^{1 \times p_{1 i}^{\prime}} R_{1 i}^{\prime}$ for $i=1, \ldots, n$.

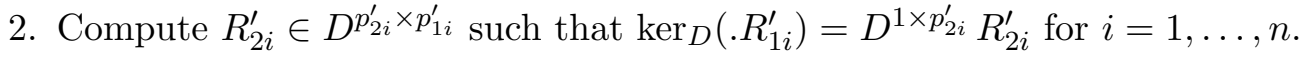

3. Left factorize $R_{1 i}$ by $R_{1 i}^{\prime}$ to get $R_{1 i}^{\prime \prime} \in D^{p_{1 i} \times p_{1 i}^{\prime}}$ such that $R_{1 i}=R_{1 i}^{\prime \prime} R_{1 i}^{\prime}$ for $i=1, \ldots, n$.

4. Compute $F_{1 i}^{\prime} \in D^{p_{1 i}^{\prime} \times p_{1(i-1)}^{\prime}}$ such that $R_{1 i}^{\prime} F_{0 i}=F_{1 i}^{\prime} R_{1(i-1)}^{\prime}$ for $i=2, \ldots, n$.

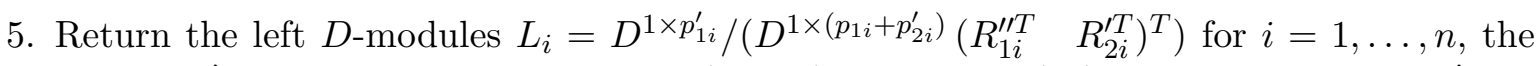
matrix $R_{11}^{\prime}$ which defines $\bar{\gamma}_{10} \in \operatorname{hom}_{D}\left(L_{1}, M\right)$ defined by $(76)$, and the matrices $F_{1(i+1)}^{\prime}$ which define $\bar{\gamma}_{(i+1) i} \in \operatorname{hom}_{D}\left(L_{i+1}, L_{i}\right)$ by 72 for $i=1, \ldots, n-1$. 
Algorithm 3 is implemented in the PurityFiltration package [45].

Using 3 of Proposition 5, we obtain the following explicit finite presentation of $\operatorname{coker} \bar{\gamma}_{(i+1) i}$ :

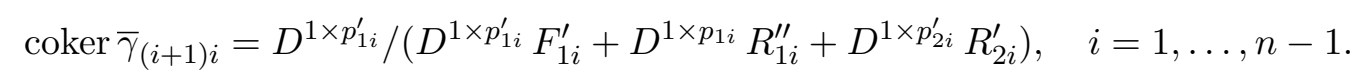

We shall denote by $\sigma_{i}: D^{1 \times p_{1 i}^{\prime}} \longrightarrow \operatorname{coker} \bar{\gamma}_{(i+1) i}$ the canonical projection onto coker $\bar{\gamma}_{(i+1) i}$.

Up to isomorphism, the short exact sequences

$$
0 \longrightarrow T_{i+1} \stackrel{\gamma_{(i+1) i}}{\longrightarrow} T_{i} \longrightarrow \operatorname{coker} \gamma_{(i+1) i} \longrightarrow 0, \quad i=1, \ldots, n-1,
$$

defined in (53) (see also (46) ) give rise to the following exact sequences:

$$
0 \longrightarrow L_{i+1} \stackrel{\bar{\gamma}_{(i+1) i}}{\longrightarrow} L_{i} \stackrel{\theta_{i}}{\longrightarrow} \operatorname{coker} \bar{\gamma}_{(i+1) i} \longrightarrow 0, \quad i=1, \ldots, n-1 .
$$

Since both $\gamma_{10}$ and $\chi_{1}$ are injective so is $\bar{\gamma}_{10}$, and $(75)$ yields the following short exact sequence

$$
0 \longrightarrow L_{1} \stackrel{\bar{\gamma}_{10}}{\longrightarrow} M \stackrel{\rho}{\longrightarrow} M / M_{1} \longrightarrow 0,
$$

where $M / M_{1} \cong D^{1 \times p_{01}} / \operatorname{ker}_{D}\left(. R_{01}\right)=D^{1 \times p_{01}} /\left(D^{1 \times p_{11}^{\prime}} R_{11}^{\prime}\right)$ (see 50$)$ ).

We recall that coker $\bar{\gamma}_{(i+1) i} \cong \operatorname{coker} \gamma_{(i+1) i} \subseteq \operatorname{ext}_{D}^{i}\left(\operatorname{ext}_{D}^{i}(M, D), D\right)$ (see (54)), and thus coker $\bar{\gamma}_{(i+1) i}$ is either zero or an $i$-pure left $D$-module when $D$ is an Auslander regular ring (see 3 of Theorem 8 and Remark 7). Exact sequences (78) and (79) will be used in Section 4.

Remark 15. Let us point out that the left $D$-modules $M_{i}$ 's can also be characterized by means of the left $D$-homomorphisms $\bar{\gamma}_{i(i-1)}$ 's. Combining (74) with $(75)$, we obtain the following commutative exact diagram:

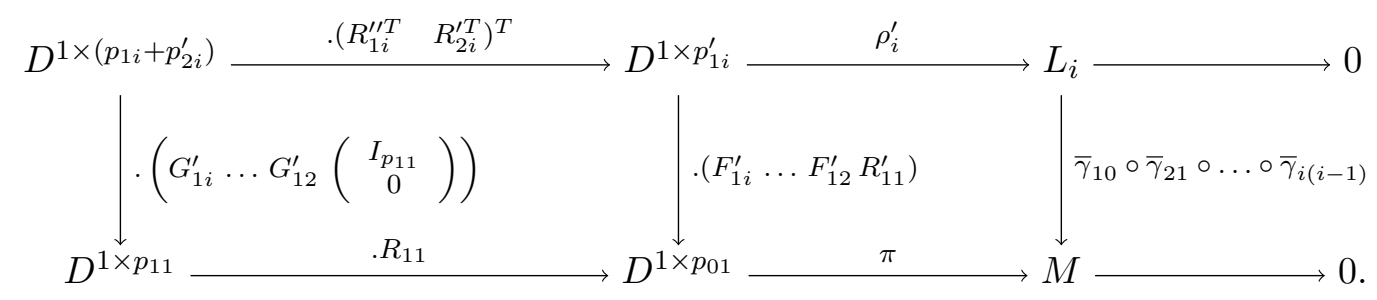

By construction (see (66)), the identity $R_{1 i}^{\prime} F_{1 i} \ldots F_{12}=F_{1 i}^{\prime} \ldots F_{12}^{\prime} R_{11}^{\prime}$ holds. Hence, using 62) and 2 of Proposition 5, we obtain:

$$
\operatorname{im}\left(\bar{\gamma}_{10} \circ \bar{\gamma}_{21} \circ \ldots \circ \bar{\gamma}_{i(i-1)}\right)=\left(D^{1 \times p_{1 i}^{\prime}}\left(F_{1 i}^{\prime} \ldots F_{12}^{\prime} R_{11}^{\prime}\right)+D^{1 \times p_{11}} R_{11}\right) /\left(D^{1 \times p_{11}} R_{11}\right)=M_{i} .
$$

Hence, the residue classes of the rows of the matrix $R_{1 i}^{\prime} F_{1 i} \ldots F_{12}=F_{1 i}^{\prime} \ldots F_{12}^{\prime} R_{11}^{\prime}$ in the left $D$-module $M=D^{1 \times p_{01}} /\left(D^{1 \times p_{11}} R_{11}\right)$ generates the left $D$-module $M_{i}$ for $i=1, \ldots, n$.

Finally, we explain an efficient way to obtain the grade filtration of a nontrivial $\operatorname{ext}_{D}^{i}(N, D)$ for $i \geq 1$. We consider the case of a right $D$-module $N$ (the case of a left $D$-module is similar). Let us first study the case of $\operatorname{ext}_{D}^{1}(N, D)$, where $N=D^{q} /\left(R D^{p}\right)$. If we introduce the Auslander transpose $M=D^{1 \times p} /\left(D^{1 \times q} R\right)$ of $N$, then the above results shows that $t_{1}(M)=\operatorname{ext}_{D}^{1}(N, D)$, and thus the grade filtration of $\operatorname{ext}_{D}^{1}(N, D)$ can be obtained by computing the grade filtration of $M$. Let us now study the case $i \geq 2$. Considering a free resolution (4) of $N$ and introducing the right $D$-module $P=D^{q_{i-1}} /\left(S_{i} D^{q_{i}}\right) \cong \operatorname{im}_{D}\left(S_{i-1}\right.$. $)$, then applying Proposition 1 to the long exact sequence $0 \longleftarrow N \stackrel{\kappa}{\longleftarrow} D^{q_{0}} \stackrel{S_{1}}{\longleftarrow} D^{q_{1}} \stackrel{S_{2}}{\longleftarrow} \ldots \stackrel{S_{i-2} .}{\longleftarrow} D^{q_{i-2}} \longleftarrow P \longleftarrow 0$, we get $\operatorname{ext}_{D}^{i}(N, D) \cong \operatorname{ext}_{D}^{1}(P, D)=t_{1}(L)$, where $L=D^{1 \times q_{i}} /\left(D^{1 \times p_{i-1}} S_{i}\right)$ is the Auslander transpose of $P$, which shows that the grade filtration of $L$ gives the grade filtration of $\operatorname{ext}_{D}^{i}(N, D)$. The corresponding algorithm is implemented in the PURITyFiltration package [45]. 


\section{Equidimensional triangularization of linear systems}

The purpose of this section is to apply Theorem 7 on Baer's extensions to the short exact sequences $(78)$ and $(79)$ to obtain a block-triangular matrix which presents the finitely generated left $D$-module $M$, and whose block-diagonal matrices are presentation matrices of the pure left $D$-modules $M_{i} / M_{i+1}$, where the $M_{i}$ 's are the left $D$-modules defined by the filtration (57) of $M$.

To simplify the exposition, we only consider the first three terms of the filtration (57) of $M$, namely, $M_{3} \subseteq M_{2} \subseteq M_{1} \subseteq M$, to obtain a presentation matrix $P$ of $M$ based on the presentation matrices of the left $D$-modules $M_{3}, M_{2} / M_{3}, M_{1} / M_{2}$ and $M / M_{1}$. If $D$ is an Auslander regular ring, then $M / M_{1}$ (resp., $M_{1} / M_{2}, M_{2} / M_{3}$ ) is 0-pure (resp., 1-pure, 2-pure). The left $D$-module $M_{3}$ satisfies $j_{D}\left(M_{3}\right) \geq 3$ but it is generally not 3-pure (it is the case if $\operatorname{gld}(D)=3$ ). But, from the clear pattern of the presentation matrix $P$, we can easily determine the general result.

We point out that the approach used here emphasizes another main advantage of our approach over the ones based on more sophisticated techniques of homological algebra. If we do no want to separate the elements of $M$ of grade number greater than or equal to $j$, then we only need to compute the first $j$ terms of the filtration (57) of $M$. But, it does not seem to be easy to stop a spectral sequence computation to only get the first steps of the grade filtration (57).

By (78) and (79), the following short exact sequences hold

$$
\begin{gathered}
0 \longrightarrow L_{3} \stackrel{\bar{\gamma}_{32}}{\longrightarrow} L_{2} \stackrel{\theta_{2}}{\longrightarrow} \text { coker } \bar{\gamma}_{32} \longrightarrow 0, \\
0 \longrightarrow L_{2} \stackrel{\bar{\gamma}_{21}}{\longrightarrow} L_{1} \stackrel{\theta_{1}}{\longrightarrow} \operatorname{coker} \bar{\gamma}_{21} \longrightarrow 0, \\
0 \longrightarrow L_{1} \stackrel{\bar{\gamma}_{10}}{\longrightarrow} M \stackrel{\rho}{\longrightarrow} M / M_{1} \longrightarrow 0,
\end{gathered}
$$

where $L_{i}$ (resp., coker $\left.\bar{\gamma}_{(i+1) i}\right)$ is defined by 64 (resp., 77$)$ ) and $M / M_{1} \cong D^{1 \times p_{01}} /\left(D^{1 \times p_{11}^{\prime}} R_{11}^{\prime}\right.$ ).

Using the definitions of $L_{2}, L_{3}$, and coker $\bar{\gamma}_{32}$ (see (65) and (77)), the following commutative exact diagram holds

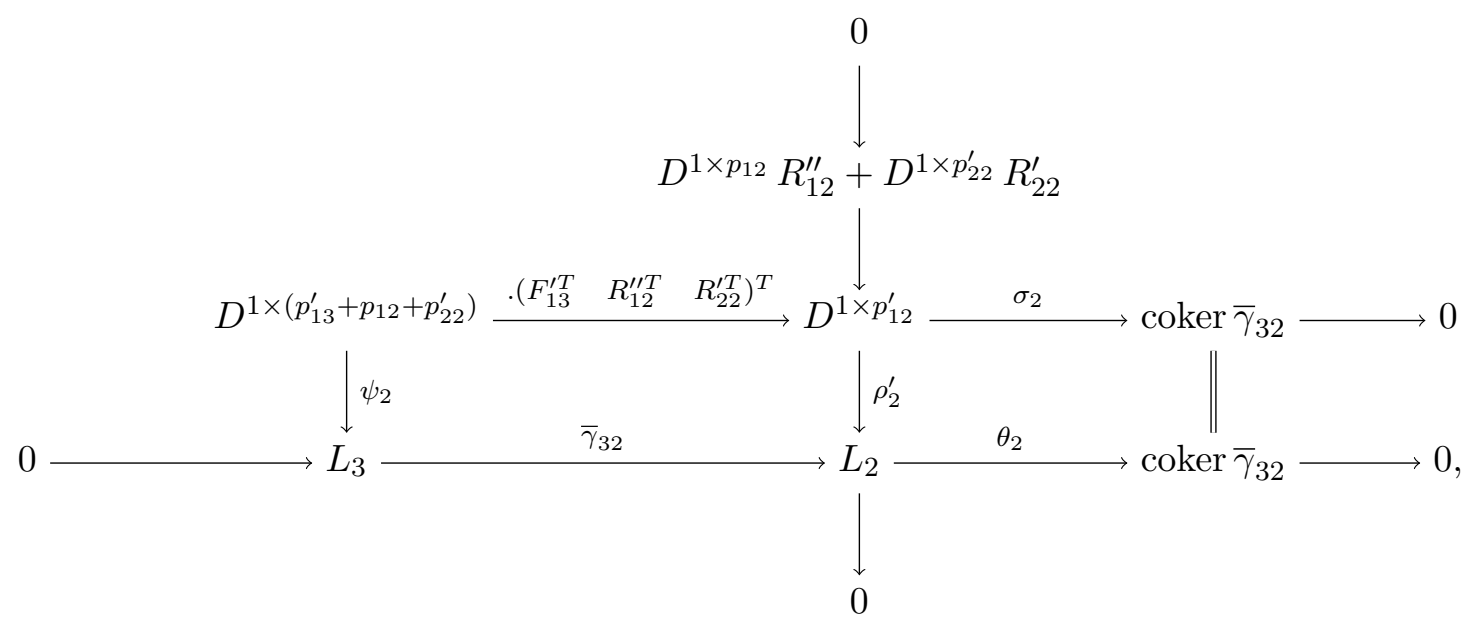

where $\psi_{2}: D^{1 \times\left(p_{13}^{\prime}+p_{12}+p_{22}^{\prime}\right)} \longrightarrow L_{3}$ is the left $D$-homomorphism defined by:

$$
\psi_{2}\left(e_{i}\right)= \begin{cases}\rho_{3}^{\prime}\left(e_{i}\right) & i=1, \ldots, p_{13}^{\prime}, \\ 0, & i=p_{13}^{\prime}+1, \ldots, p_{13}^{\prime}+p_{12}+p_{22}^{\prime}\end{cases}
$$


Applying Theorem 7 to the first short exact sequence of $(80)$ with the matrix

$$
A=\left(I_{p_{13}^{\prime}}^{T} \quad 0^{T} \quad 0^{T}\right)^{T} \in D^{\left(p_{13}^{\prime}+p_{12}+p_{22}^{\prime}\right) \times p_{13}^{\prime}}
$$

(see Corollary 2), we obtain the following characterization of the left $D$-module $L_{2}$ in terms of the presentations of the left $D$-modules $L_{3}$ and coker $\bar{\gamma}_{32}$.

Proposition 9. With the previous hypotheses and notations, let us consider

$$
Q_{2}=\left(\begin{array}{c}
R_{12}^{\prime \prime} \\
R_{22}^{\prime}
\end{array}\right) \in D^{\left(p_{12}+p_{22}^{\prime}\right) \times p_{12}^{\prime},} \quad P_{2}=\left(\begin{array}{cc}
F_{13}^{\prime} & -I_{p_{13}^{\prime}} \\
R_{12}^{\prime \prime} & 0 \\
R_{22}^{\prime} & 0 \\
0 & R_{13}^{\prime \prime} \\
0 & R_{23}^{\prime}
\end{array}\right) \in D^{\left(p_{13}^{\prime}+p_{12}+p_{22}^{\prime}+p_{13}+p_{23}^{\prime}\right) \times\left(p_{12}^{\prime}+p_{13}^{\prime}\right)},
$$

and the following two finitely presented left D-modules:

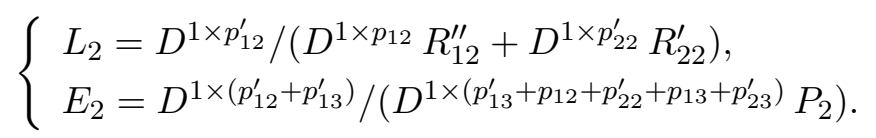

If $\varrho_{2}: D^{1 \times\left(p_{12}^{\prime}+p_{13}^{\prime}\right)} \longrightarrow E_{2}$ is the canonical projection onto $E_{2}$, then we have $E_{2} \cong L_{2}$, where the left D-isomorphism is defined by:

$$
\begin{aligned}
& \phi_{2}: L_{2} \longrightarrow E_{2} \quad \phi_{2}^{-1}: E_{2} \longrightarrow L_{2}
\end{aligned}
$$

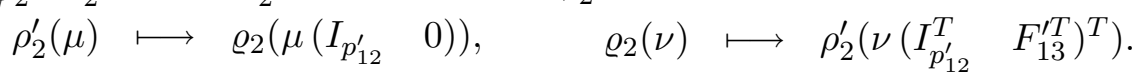

Proof. Let us consider the following matrices:

$$
\begin{gathered}
V_{2}=\left(\begin{array}{ll}
I_{p_{12}^{\prime}} & 0
\end{array}\right) \in D^{p_{12}^{\prime} \times\left(p_{12}^{\prime}+p_{13}^{\prime}\right)}, \quad W_{2}=\left(\begin{array}{ccccc}
0 & I_{p_{12}} & 0 & 0 & 0 \\
0 & 0 & I_{p_{22}^{\prime}} & 0 & 0
\end{array}\right) \in D^{\left(p_{12}+p_{22}^{\prime}\right) \times\left(p_{13}^{\prime}+p_{12}+p_{22}^{\prime}+p_{13}+p_{23}^{\prime}\right),} \\
X_{2}=\left(\begin{array}{c}
I_{p_{12}^{\prime}} \\
F_{13}^{\prime}
\end{array}\right) \in D^{\left(p_{12}^{\prime}+p_{13}^{\prime}\right) \times p_{12}^{\prime}}, \quad Y_{2}=\left(\begin{array}{ccc}
0 & 0 \\
I_{p_{12}} & 0 \\
0 & I_{p_{22}^{\prime}} \\
F_{13} & -X_{22} \\
0 & F_{23}^{\prime}
\end{array}\right) \in D^{\left(p_{13}^{\prime}+p_{12}+p_{22}^{\prime}+p_{13}+p_{23}^{\prime}\right) \times\left(p_{12}+p_{22}^{\prime}\right) .}
\end{gathered}
$$

Using (67) and (70), we can easily check that $Q_{2} V_{2}=W_{2} P_{2}$ (resp., $P_{2} X_{2}=Y_{2} Q_{2}$ ), which by Proposition 5 induces $\phi_{2} \in \operatorname{hom}_{D}\left(L_{2}, E_{2}\right)$ defined by (81) (resp., $\psi_{2} \in \operatorname{hom}_{D}\left(E_{2}, L_{2}\right)$ ). Since $V_{2} X_{2}=I_{p_{12}^{\prime}}$, we get $\psi_{2} \circ \phi_{2}=\mathrm{id}_{L_{2}}$, which shows that $\phi_{2}$ is injective. Using 3 of Proposition 5 , the left $D$-module coker $\phi_{2}$ is finitely presented by the matrix $\left(\begin{array}{lll}V_{2}^{T} & P_{2}^{T}\end{array}\right)^{T}$, which admits the following left inverse over $D$ :

$$
\left(\begin{array}{ccccc}
I_{p_{12}^{\prime}} & 0 & 0 & 0 & 0 \\
F_{13}^{\prime} & -I_{p_{13}^{\prime}} & 0 & 0 & 0
\end{array}\right) \in D^{\left(p_{12}^{\prime}+p_{13}^{\prime}\right) \times\left(p_{12}^{\prime}+p_{13}^{\prime}+p_{12}+p_{22}^{\prime}+p_{13}+p_{23}^{\prime}\right)} .
$$

Hence, coker $\phi_{2}=0$, i.e., $\phi_{2}$ is surjective, and thus $\phi_{2}$ is an isomorphism, $E_{2} \cong L_{2}$, and $\phi_{2}^{-1}=\psi_{2}$. 
Using the left $D$-isomorphism $\phi_{2}^{-1}: E_{2} \longrightarrow L_{2}$ defined by 81 , the second short exact sequence of 80 yields the following short exact sequence

$$
0 \longrightarrow E_{2} \stackrel{\bar{\gamma}_{21} \circ \phi_{2}^{-1}}{\longrightarrow} L_{1} \stackrel{\theta_{1}}{\longrightarrow} \operatorname{coker} \bar{\gamma}_{21} \longrightarrow 0
$$

where using 72 , the left $D$-homomorphism $\bar{\gamma}_{21} \circ \phi_{2}^{-1}: E_{2} \longrightarrow L_{1}$ is defined by:

$$
\forall \nu \in D^{1 \times\left(p_{12}^{\prime}+p_{13}^{\prime}\right)}, \quad\left(\bar{\gamma}_{21} \circ \phi_{2}^{-1}\right)\left(\varrho_{2}(\nu)\right)=\bar{\gamma}_{21}\left(\rho_{2}^{\prime}\left(\nu\left(\begin{array}{c}
I_{p_{12}^{\prime}} \\
F_{13}^{\prime}
\end{array}\right)\right)\right)=\rho_{1}^{\prime}\left(\nu\left(\begin{array}{c}
F_{12}^{\prime} \\
F_{13}^{\prime} F_{12}^{\prime}
\end{array}\right)\right) .
$$

Using the definitions of $L_{1}, E_{2}$, and coker $\bar{\gamma}_{21}$ (see (65), Proposition 9 and (77)), we get the commutative exact diagram

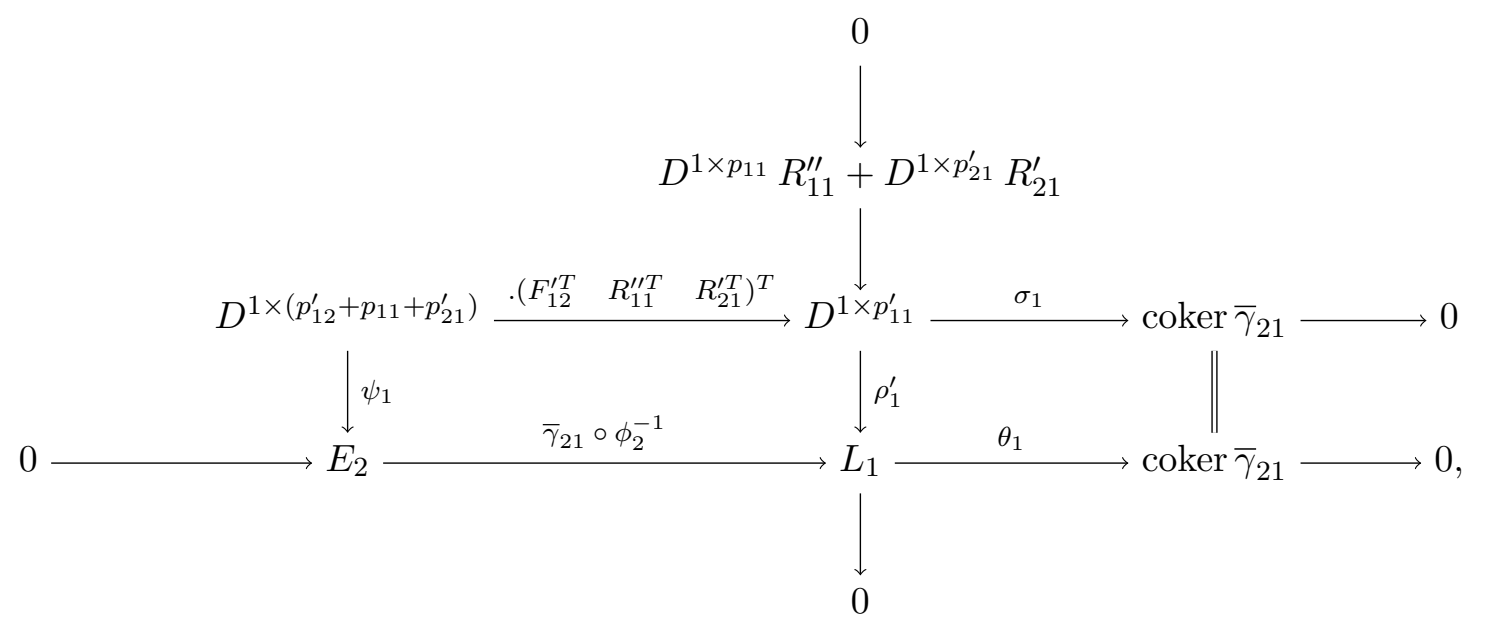

where $\psi_{1}: D^{1 \times\left(p_{12}^{\prime}+p_{11}+p_{21}^{\prime}\right)} \longrightarrow E_{2}$ is the left $D$-homomorphism defined by

$$
\psi_{1}\left(f_{j}\right)= \begin{cases}\varrho_{2}\left(f_{j} F\right), & j=1, \ldots, p_{12}^{\prime}, \\ 0, & j=p_{12}^{\prime}+1, \ldots, p_{12}^{\prime}+p_{11}+p_{21}^{\prime}\end{cases}
$$

$\left\{f_{j}\right\}_{j=1, \ldots, p_{12}^{\prime}+p_{11}+p_{21}^{\prime}}$ is the standard basis of $D^{1 \times\left(p_{12}^{\prime}+p_{11}+p_{21}^{\prime}\right)}$ and:

$$
F=\left(\begin{array}{cc}
I_{p_{12}^{\prime}} & 0 \\
0 & 0 \\
0 & 0
\end{array}\right) \in D^{\left(p_{12}^{\prime}+p_{11}+p_{21}^{\prime}\right) \times\left(p_{12}^{\prime}+p_{13}^{\prime}\right)}
$$

Applying Theorem 7 to the short exact sequence 82 ) with the matrix $A=F$ (see Corollary 2), we obtain the following proposition. 
Proposition 10. With the hypotheses of Proposition 9 and the previous notations, let us consider the following two matrices

$$
\begin{aligned}
P_{1} & =\left(\begin{array}{ccc}
F_{12}^{\prime} & -I_{p_{12}^{\prime}} & 0 \\
R_{11}^{\prime \prime} & 0 & 0 \\
R_{21}^{\prime} & 0 & 0 \\
0 & F_{13}^{\prime} & -I_{p_{13}^{\prime}} \\
0 & R_{12}^{\prime \prime} & 0 \\
0 & R_{22}^{\prime} & 0 \\
0 & 0 & R_{13}^{\prime \prime} \\
0 & 0 & R_{23}^{\prime}
\end{array}\right) \in D^{\left(p_{12}^{\prime}+p_{11}+p_{21}^{\prime}+p_{13}^{\prime}+p_{12}+p_{22}^{\prime}+p_{13}+p_{23}^{\prime}\right) \times\left(p_{11}^{\prime}+p_{12}^{\prime}+p_{13}^{\prime}\right)} \\
Q_{1} & =\left(\begin{array}{c}
R_{11}^{\prime \prime} \\
R_{21}^{\prime}
\end{array}\right) \in D^{\left(p_{11}+p_{21}^{\prime}\right) \times p_{11}^{\prime},}
\end{aligned}
$$

and the following two finitely presented left D-modules:

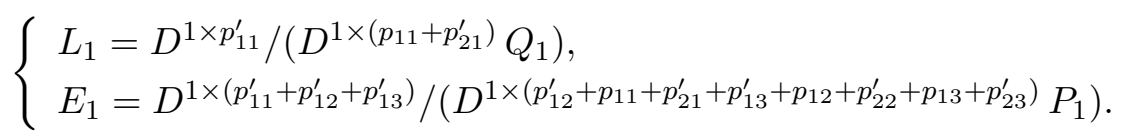

If $\varrho_{1}: D^{1 \times\left(p_{11}^{\prime}+p_{12}^{\prime}+p_{13}^{\prime}\right)} \longrightarrow E_{1}$ is the canonical projection onto $E_{1}$, then we have $E_{1} \cong L_{1}$, where the left $D$-isomorphism is defined by:

$$
\begin{aligned}
& \phi_{1}^{-1}: E_{1} \quad \longrightarrow \quad L_{1}
\end{aligned}
$$

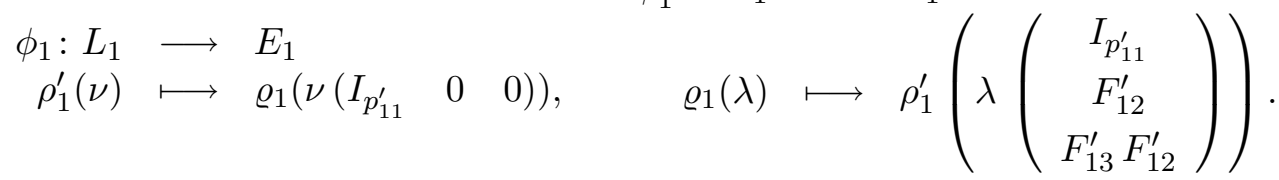

Finally, we have $L_{1} \cong M_{1}$, with the following left D-isomorphisms:

$$
\begin{aligned}
& \chi_{1}: L_{1} \longrightarrow M_{1} \quad \chi_{1}^{-1}: M_{1} \longrightarrow L_{1} \\
& \rho_{1}^{\prime}(\nu) \longmapsto \pi\left(\nu R_{11}^{\prime}\right), \quad \pi\left(\nu R_{11}^{\prime}\right) \longmapsto \rho_{1}^{\prime}(\nu) .
\end{aligned}
$$

Proof. Let us consider the following matrices:

$$
\begin{aligned}
& V_{1}=\left(\begin{array}{lll}
I_{p_{11}^{\prime}} & 0 & 0
\end{array}\right) \in D^{p_{11}^{\prime} \times\left(p_{11}^{\prime}+p_{12}^{\prime}+p_{13}^{\prime}\right)}, \quad X_{1}=\left(I_{p_{11}^{\prime}}^{T} \quad F_{12}^{\prime T} \quad\left(F_{13}^{\prime} F_{12}^{\prime}\right)^{T}\right)^{T} \in D^{\left(p_{11}^{\prime}+p_{12}^{\prime}+p_{13}^{\prime}\right) \times p_{11}^{\prime}}, \\
& W_{1}=\left(\begin{array}{cccccccc}
0 & I_{p_{11}} & 0 & 0 & 0 & 0 & 0 & 0 \\
0 & 0 & I_{p_{21}^{\prime}} & 0 & 0 & 0 & 0 & 0
\end{array}\right) \in D^{\left(p_{11}+p_{21}^{\prime}\right) \times\left(p_{12}^{\prime}+p_{11}+p_{21}^{\prime}+p_{13}^{\prime}+p_{12}+p_{22}^{\prime}+p_{13}+p_{23}^{\prime}\right)}, \\
& Y_{1}=\left(\begin{array}{cc}
0 & 0 \\
I_{p_{11}} & 0 \\
0 & I_{p_{21}^{\prime}} \\
0 & 0 \\
I_{p_{11}} & -X_{12} \\
0 & F_{22}^{\prime} \\
F_{13} & -F_{13} X_{12}-X_{22} F_{22}^{\prime} \\
0 & F_{23}^{\prime} F_{22}^{\prime}
\end{array}\right) \in D^{\left(p_{12}^{\prime}+p_{11}+p_{21}^{\prime}+p_{13}^{\prime}+p_{12}+p_{22}^{\prime}+p_{13}+p_{23}^{\prime}\right) \times\left(p_{11}+p_{21}^{\prime}\right)} \text {. }
\end{aligned}
$$

$\mathrm{RR} \mathrm{n}^{\circ} 7769$ 
Using (67) and (70), we can check that $Q_{1} V_{1}=W_{1} P_{1}$ (resp., $P_{1} X_{1}=Y_{1} Q_{1}$ ), which by Proposition 5 induces $\phi_{1} \in \operatorname{hom}_{D}\left(L_{1}, E_{1}\right)$ defined by (83) (resp., $\psi_{1} \in \operatorname{hom}_{D}\left(E_{1}, L_{1}\right)$ ). Since $V_{1} X_{1}=I_{p_{11}^{\prime}}$, we get $\psi_{1} \circ \phi_{1}=\mathrm{id}_{L_{1}}$, which shows that $\phi_{1}$ is injective. Using 3 of Proposition 5 , the left $D$-module coker $\phi_{1}$ is finitely presented by the matrix $\left(\begin{array}{lll}V_{1}^{T} & P_{1}^{T}\end{array}\right)^{T}$, which admits the following left inverse over $D$ :

$$
\left(\begin{array}{ccccccccc}
I_{p_{11}^{\prime}} & 0 & 0 & 0 & 0 & 0 & 0 & 0 & 0 \\
F_{12}^{\prime} & -I_{p_{12}^{\prime}} & 0 & 0 & 0 & 0 & 0 & 0 & 0 \\
F_{13}^{\prime} F_{12}^{\prime} & -F_{13}^{\prime} & 0 & 0 & -I_{p_{13}^{\prime}} & 0 & 0 & 0 & 0
\end{array}\right) \in D^{\left(p_{11}^{\prime}+p_{12}^{\prime}+p_{13}^{\prime}\right) \times\left(p_{11}^{\prime}+p_{12}^{\prime}+p_{11}+p_{21}^{\prime}+p_{13}^{\prime}+p_{12}+p_{22}^{\prime}+p_{13}+p_{23}^{\prime}\right)} .
$$

Hence, coker $\phi_{1}=0$, i.e., $\phi_{1}$ is surjective, and thus, $\phi_{1}$ is an isomorphism, $E_{1} \cong L_{1}$, and $\phi_{1}^{-1}=\psi_{1}$. Finally, the last result of Proposition 10 was already proved in Remark 15 .

Using Proposition 10 and Remark $15, \bar{\gamma}_{10} \circ \phi_{1}^{-1}: E_{1} \longrightarrow M_{1}$ is then defined by:

$$
\left(\chi_{1} \circ \phi_{1}^{-1}\right)\left(\varrho_{1}(\lambda)\right)=\pi\left(\lambda\left(\begin{array}{c}
R_{11}^{\prime} \\
F_{12}^{\prime} R_{11}^{\prime} \\
F_{13}^{\prime} F_{12}^{\prime} R_{11}^{\prime}
\end{array}\right)\right) .
$$

Then, the third short exact sequence 80 yields the following one:

$$
0 \longrightarrow E_{1} \stackrel{\bar{\gamma}_{10} \circ \phi_{1}^{-1}}{\longrightarrow} M \stackrel{\rho}{\longrightarrow} M / M_{1} \longrightarrow 0 .
$$

Now, we can easily check that the following commutative exact diagram holds

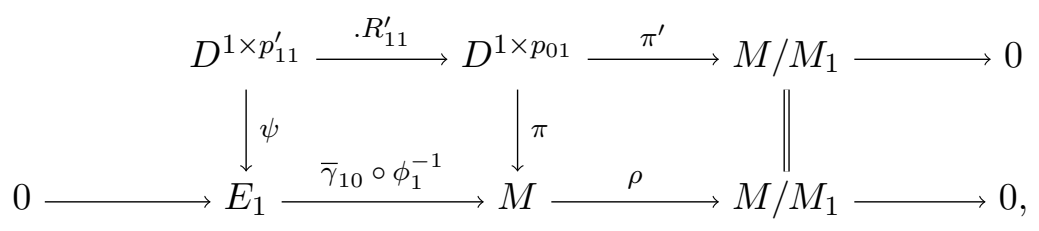

where $\psi: D^{1 \times p_{11}^{\prime}} \longrightarrow E_{1}$ is defined by $\psi\left(g_{k}\right)=\varrho_{1}\left(g_{k}\left(I_{p_{11}^{\prime}} \quad 0 \quad 0\right)\right)$, and $\left\{g_{k}\right\}_{k=1, \ldots, p_{11}^{\prime}}$ is the standard basis of $D^{1 \times p_{11}^{\prime}}$. Then, we can apply Theorem 7 to the short exact sequence 84 with $A=\left(\begin{array}{lll}I_{p_{11}^{\prime}} & 0 & 0\end{array}\right) \in D^{p_{11}^{\prime} \times\left(p_{11}^{\prime}+p_{12}^{\prime}+p_{13}^{\prime}\right)}$ (see Corollary 2$)$ to get the following theorem.

Theorem 11. Let $D$ be a noetherian domain which satisfies (38). With the previous notations, let us consider the following matrix

$$
P=\left(\begin{array}{cccc}
R_{11}^{\prime} & -I_{p_{11}^{\prime}} & 0 & 0 \\
0 & F_{12}^{\prime} & -I_{p_{12}^{\prime}} & 0 \\
0 & R_{11}^{\prime \prime} & 0 & 0 \\
0 & R_{21}^{\prime} & 0 & 0 \\
0 & 0 & F_{13}^{\prime} & -I_{p_{13}^{\prime}} \\
0 & 0 & R_{12}^{\prime \prime} & 0 \\
0 & 0 & R_{22}^{\prime} & 0 \\
0 & 0 & 0 & R_{13}^{\prime \prime} \\
0 & 0 & 0 & R_{23}^{\prime}
\end{array}\right) \in D^{\left(p_{11}^{\prime}+p_{12}^{\prime}+p_{11}+p_{21}^{\prime}+p_{13}^{\prime}+p_{12}+p_{22}^{\prime}+p_{13}+p_{23}^{\prime}\right) \times\left(p_{01}+p_{11}^{\prime}+p_{12}^{\prime}+p_{13}^{\prime}\right)}
$$


and the following two finitely presented left D-modules:

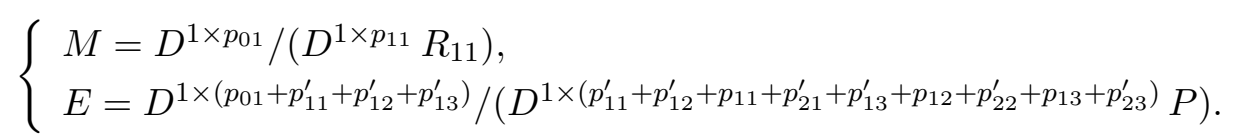

If $\varrho: D^{1 \times\left(p_{01}+p_{11}^{\prime}+p_{12}^{\prime}+p_{13}^{\prime}\right)} \longrightarrow E$ is the canonical projection onto $E$, then we have $E \cong M$, where the left D-isomorphism is defined by:

$$
\begin{aligned}
& \phi^{-1}: E \longrightarrow M
\end{aligned}
$$

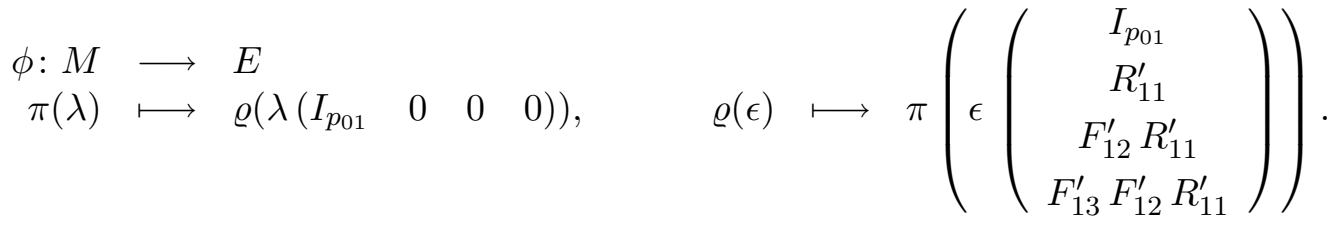

Proof. Let us consider the following matrices:

$$
\begin{aligned}
& V=\left(\begin{array}{llll}
I_{p_{01}} & 0 & 0 & 0
\end{array}\right) \in D^{p_{01} \times\left(p_{01}+p_{11}^{\prime}+p_{12}^{\prime}+p_{13}^{\prime}\right)}, \\
& W=\left(\begin{array}{lllllllll}
R_{11}^{\prime \prime} & 0 & I_{p_{11}^{\prime}} & 0 & 0 & 0 & 0 & 0 & 0
\end{array}\right) \in D^{p_{11} \times\left(p_{11}^{\prime}+p_{12}^{\prime}+p_{11}+p_{21}^{\prime}+p_{13}^{\prime}+p_{12}+p_{22}^{\prime}+p_{13}+p_{23}^{\prime}\right)}, \\
& X=\left(\begin{array}{c}
I_{p_{01}} \\
R_{11}^{\prime} \\
F_{12}^{\prime} R_{11}^{\prime} \\
F_{13}^{\prime} F_{12}^{\prime} R_{11}^{\prime}
\end{array}\right) \in D^{\left(p_{01}+p_{11}^{\prime}+p_{12}^{\prime}+p_{13}^{\prime}\right) \times p_{01}}, \\
& Y=\left(\begin{array}{c}
0 \\
0 \\
I_{p_{11}} \\
0 \\
0 \\
I_{p_{11}} \\
0 \\
F_{13} \\
0
\end{array}\right) \in D^{\left(p_{11}^{\prime}+p_{12}^{\prime}+p_{11}+p_{21}^{\prime}+p_{13}^{\prime}+p_{12}+p_{22}^{\prime}+p_{13}+p_{23}^{\prime}\right) \times p_{11}} .
\end{aligned}
$$

Using (67) and (70), we can check that $R_{11} V=W P$ (resp., $P X=Y R_{11}$ ), which by Proposition 5 induces $\phi \in \operatorname{hom}_{D}(M, E)$ defined by (85) (resp., $\psi \in \operatorname{hom}_{D}(E, M)$ ). Moreover, since $V X=I_{p_{01}}$, we get $\psi \circ \phi=\mathrm{id}_{M}$, which shows that $\phi$ is injective. Using 3 of Proposition 5 , the left $D$-module coker $\phi$ is finitely presented by the matrix $\left(\begin{array}{lll}V^{T} & P^{T}\end{array}\right)^{T}$, which admits the following left inverse over $D$ :

$$
\begin{aligned}
& \left(\begin{array}{cccccccccc}
I_{p_{01}} & 0 & 0 & 0 & 0 & 0 & 0 & 0 & 0 & 0 \\
R_{11}^{\prime} & -I_{p_{11}^{\prime}} & 0 & 0 & 0 & 0 & 0 & 0 & 0 & 0 \\
F_{12}^{\prime} R_{11}^{\prime} & -F_{12}^{\prime} & -I_{p_{12}^{\prime}} & 0 & 0 & 0 & 0 & 0 & 0 & 0 \\
F_{13}^{\prime} F_{12}^{\prime} R_{11}^{\prime} & -F_{13}^{\prime} F_{12}^{\prime} & -F_{13}^{\prime} & 0 & 0 & -I_{p_{13}^{\prime}} & 0 & 0 & 0 & 0
\end{array}\right) \\
& \in D^{\left(p_{01}+p_{11}^{\prime}+p_{12}^{\prime}+p_{11}+p_{21}^{\prime}+p_{13}^{\prime}+p_{12}+p_{22}^{\prime}+p_{13}+p_{23}^{\prime}\right) \times\left(p_{01}+p_{11}^{\prime}+p_{12}^{\prime}+p_{13}^{\prime}\right)} .
\end{aligned}
$$

Hence, coker $\phi=0$, i.e., $\phi$ is surjective, and thus, $\phi$ is an isomorphism, $E \cong M$, and $\phi^{-1}=\psi$. 
We note that $\sqrt{70}$ for $i=1$ and $F_{12}=I_{p_{11}}$ yield the following identity:

$$
R_{11}^{\prime \prime}=R_{12}^{\prime \prime} F_{12}^{\prime}+X_{12} R_{21}^{\prime} \text {. }
$$

Since the third column of $P$ contains $R_{12}^{\prime \prime}$, the third row of $P$ containing the matrix $R_{11}^{\prime \prime}$ can be removed. We then obtain the following straightforward corollary of Theorem 11.

Corollary 5. With the hypotheses and the notations of Theorem 11, if

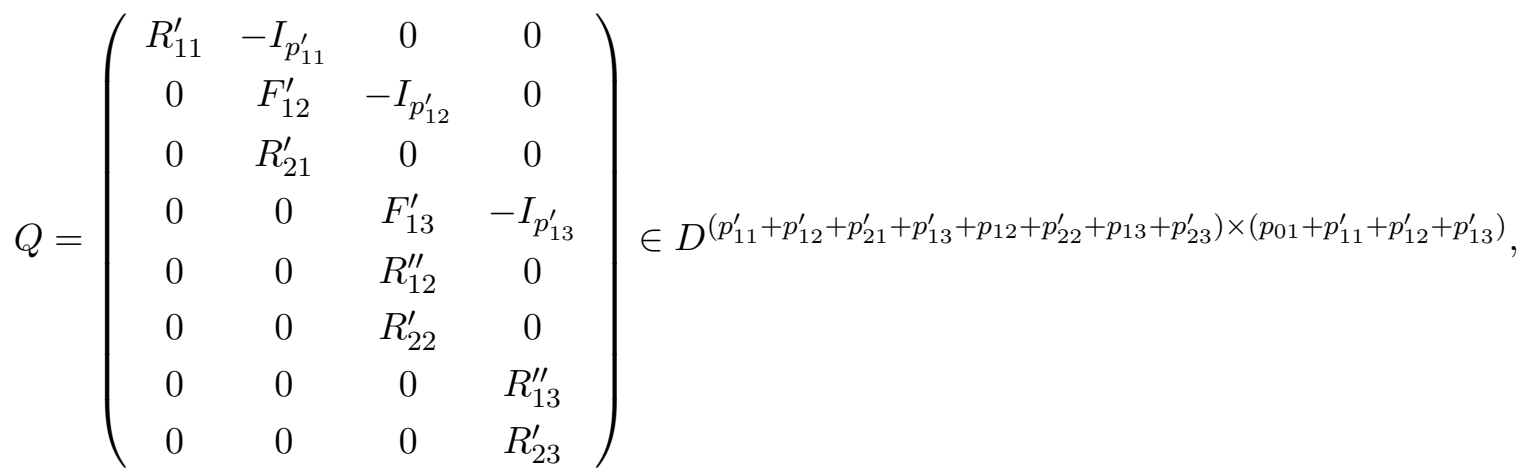

then we have

$$
\begin{aligned}
M & =D^{1 \times p_{01}} /\left(D^{1 \times p_{11}} R_{11}\right) \\
& \cong E=D^{1 \times\left(p_{01}+p_{11}^{\prime}+p_{12}^{\prime}+p_{13}^{\prime}\right)} /\left(D^{1 \times\left(p_{11}^{\prime}+p_{12}^{\prime}+p_{21}^{\prime}+p_{13}^{\prime}+p_{12}+p_{22}^{\prime}+p_{13}+p_{23}^{\prime}\right)} Q\right),
\end{aligned}
$$

where the isomorphism is defined by 85).

Let $\mathcal{F}$ be a left $D$-module. Then, $M \cong E$ and Theorem 1 imply that $\operatorname{ker}_{\mathcal{F}}\left(R_{11}.\right) \cong \operatorname{ker}_{\mathcal{F}}(P)=$. $\operatorname{ker}_{\mathcal{F}}(Q$.$) . Applying the functor \operatorname{hom}_{D}(\cdot, \mathcal{F})$ to the diagram defined in Figure 1, we obtain the diagram of abelian groups defined in Figure 2 formed by horizontal complexes of abelian groups. More precisely, using (85) and $R=R_{11}$, we obtain the following corollary.

Corollary 6. If $D$ is a noetherian domain which satisfies (38), $R \in D^{q \times p}$, and $\mathcal{F}$ a left $D$ module, then

$$
\operatorname{ker}_{\mathcal{F}}(R .) \cong \operatorname{ker}_{\mathcal{F}}(Q .),
$$

i.e., the following system equivalence holds

$$
R \eta=0 \Leftrightarrow\left\{\begin{array}{l}
R_{11}^{\prime} \zeta-\tau_{1}=0 \\
F_{12}^{\prime} \tau_{1}-\tau_{2}=0 \\
R_{21}^{\prime} \tau_{1}=0 \\
F_{13}^{\prime} \tau_{2}-\tau_{3}=0 \\
R_{12}^{\prime \prime} \tau_{2}=0 \\
R_{22}^{\prime} \tau_{2}=0 \\
R_{13}^{\prime \prime} \tau_{3}=0 \\
R_{23}^{\prime} \tau_{3}=0
\end{array}\right.
$$

under the following invertible transformations:

$$
\begin{aligned}
& \gamma: \operatorname{ker}_{\mathcal{F}}(Q .) \longrightarrow \operatorname{ker}_{\mathcal{F}}(R .) \quad \gamma^{-1}: \operatorname{ker}_{\mathcal{F}}(R .) \longrightarrow \operatorname{ker}_{\mathcal{F}}(Q .) \\
& \left(\begin{array}{c}
\zeta \\
\tau_{1} \\
\tau_{2} \\
\tau_{3}
\end{array}\right) \longmapsto \eta=\zeta, \quad \eta \longmapsto\left(\begin{array}{c}
\zeta \\
\tau_{1} \\
\tau_{2} \\
\tau_{3}
\end{array}\right)=\left(\begin{array}{c}
I_{p_{01}} \\
R_{11}^{\prime} \\
F_{12}^{\prime} R_{11}^{\prime} \\
F_{13}^{\prime} F_{12}^{\prime} R_{11}^{\prime}
\end{array}\right) \eta \text {. }
\end{aligned}
$$




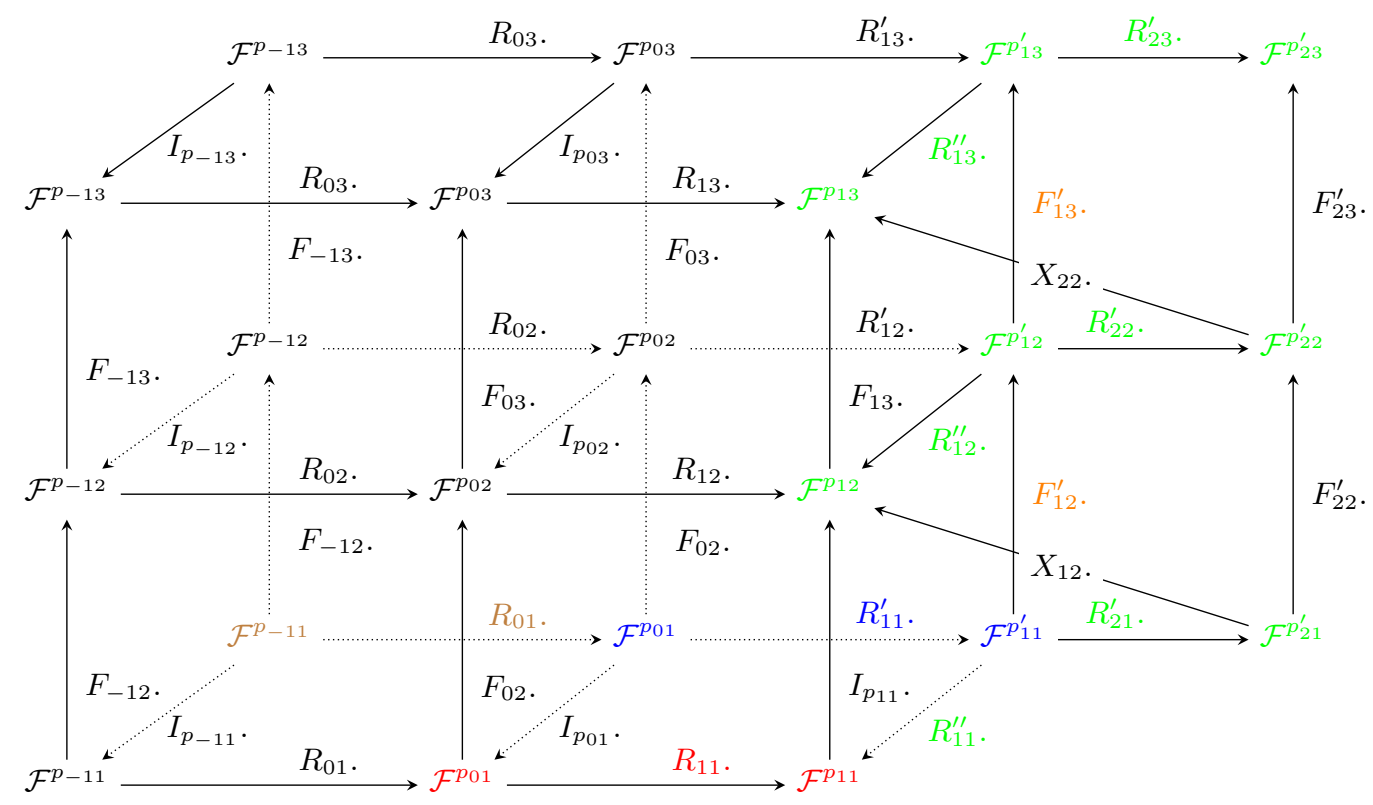

Figure 2: Dual of Figure 1

Remark 16. Let $D$ be both an Auslander regular ring and a Cohen-Macaulay ring. If we set

$$
S_{0}=R_{11}^{\prime}, \quad S_{1}=\left(\begin{array}{c}
F_{12}^{\prime} \\
R_{11}^{\prime \prime} \\
R_{21}^{\prime}
\end{array}\right), \quad S_{1}^{\prime}=\left(\begin{array}{c}
F_{12}^{\prime} \\
R_{21}^{\prime}
\end{array}\right), \quad S_{2}=\left(\begin{array}{c}
F_{13}^{\prime} \\
R_{12}^{\prime \prime} \\
R_{22}^{\prime}
\end{array}\right), \quad S_{3}=\left(\begin{array}{c}
R_{13}^{\prime \prime} \\
R_{23}^{\prime}
\end{array}\right),
$$

then:

1. $\operatorname{ker}_{\mathcal{F}}\left(S_{3}.\right) \cong \operatorname{hom}_{D}\left(L_{3}, \mathcal{F}\right) \cong \operatorname{hom}_{D}\left(\operatorname{ext}_{D}^{3}\left(N_{33}, D\right), \mathcal{F}\right)$ is either 0 or has dimension less than or equal to $\operatorname{dim}(D)-3$,

2. $\operatorname{ker}_{\mathcal{F}}\left(S_{2}.\right) \cong \operatorname{hom}_{D}\left(\operatorname{coker} \bar{\gamma}_{32}, \mathcal{F}\right) \cong \operatorname{hom}_{D}\left(\operatorname{coker} \gamma_{32}, \mathcal{F}\right)$ has dimension $\operatorname{dim}(D)-2$ when it is nonzero,

3. $\operatorname{ker}_{\mathcal{F}}\left(S_{1}.\right)=\operatorname{ker}_{\mathcal{F}}\left(S_{1}^{\prime}.\right) \cong \operatorname{hom}_{D}\left(\operatorname{coker} \bar{\gamma}_{21}, \mathcal{F}\right) \cong \operatorname{hom}_{D}\left(\operatorname{coker} \gamma_{21}, \mathcal{F}\right)$ is either 0 or has dimension $\operatorname{dim}(D)-1$,

4. $\operatorname{ker}_{\mathcal{F}}\left(S_{0}.\right) \cong \operatorname{hom}_{D}\left(M / M_{1}, \mathcal{F}\right)$ has dimension $\operatorname{dim}(D)$ when it is nonzero.

If $R_{3}$ has full row rank, i.e., $\operatorname{ker}_{D}\left(. R_{3}\right)=0$, then $N_{33} \cong \operatorname{ext}_{D}^{3}(M, D)$, and thus $\operatorname{ext}_{D}^{3}\left(N_{33}, D\right) \cong$ $\operatorname{ext}_{D}^{3}\left(\operatorname{ext}_{D}^{3}(M, D), D\right)$, and $\operatorname{ker}_{\mathcal{F}}\left(S_{3}\right.$. $)$ has $\operatorname{dim}(D)-3$ when it is nonzero.

The solution of the linear system $\operatorname{ker}_{\mathcal{F}}(R$. $)$ can then be obtained by integrating the linear system $\operatorname{ker}_{\mathcal{F}}(Q$.$) , i.e., by integrating in cascade the linear system \operatorname{ker}_{\mathcal{F}}\left(S_{3}\right.$.) of dimension less than or equal to $\operatorname{dim}(D)-3$, then the inhomogeneous linear systems of dimension respectively $\operatorname{dim}(D)-2, \operatorname{dim}(D)-1$ and $\operatorname{dim}(D)$. Finally, if $\mathcal{F}$ is an injective left $D$-module, then the linear system $\operatorname{ker}_{\mathcal{F}}\left(R_{11}^{\prime}.\right)$ of dimension $\operatorname{dim}(D)$ is parametrizable and $\operatorname{ker}_{\mathcal{F}}\left(R_{11}^{\prime}.\right)=R_{01} \mathcal{F}^{p_{-11}}$. 
Example 6. Let us consider an example studied by Janet and considered again in 38 defined by the $D=\mathbb{Q}\left[\partial_{1}, \partial_{2}, \partial_{3}\right]$-module $M=D^{1 \times 4} /\left(D^{1 \times 6} R\right)$ finitely presented by the following matrix:

$$
R=\left(\begin{array}{cccc}
0 & -2 \partial_{1} & \partial_{3}-2 \partial_{2}-\partial_{1} & -1 \\
0 & \partial_{3}-2 \partial_{1} & 2 \partial_{2}-3 \partial_{1} & 1 \\
\partial_{3} & -6 \partial_{1} & -2 \partial_{2}-5 \partial_{1} & -1 \\
0 & \partial_{2}-\partial_{1} & \partial_{2}-\partial_{1} & 0 \\
\partial_{2} & -\partial_{1} & -\partial_{2}-\partial_{1} & 0 \\
\partial_{1} & -\partial_{1} & -2 \partial_{1} & 0
\end{array}\right)
$$

The $D$-module $M$ admits the following finite free resolution:

$$
\begin{aligned}
& 0 \longleftarrow M \stackrel{\pi}{\longleftarrow} D^{1 \times 4} \stackrel{. R}{\longleftarrow} D^{1 \times 6} \stackrel{. R_{2}}{\longleftarrow} D^{1 \times 4} \stackrel{. R_{3}}{\longleftarrow} D \longleftarrow 0, \\
& R_{2}=\left(\begin{array}{cccccc}
2 \partial_{2} & \partial_{2} & -\partial_{2} & -\partial_{3} & \partial_{3} & 0 \\
2 \partial_{1} & \partial_{2} & -2 \partial_{1}+\partial_{2} & -\partial_{3} & 8 \partial_{1}-\partial_{3} & -8 \partial_{2}+2 \partial_{3} \\
0 & \partial_{1}-\partial_{2} & \partial_{1}-\partial_{2} & \partial_{3} & -8 \partial_{1}+\partial_{3} & 8 \partial_{2}-\partial_{3} \\
0 & 0 & 0 & \partial_{1} & -\partial_{1} & \partial_{2}
\end{array}\right), \\
& R_{3}=\left(\begin{array}{llll}
\partial_{1} & \partial_{2} & -\partial_{2} & \partial_{3}
\end{array}\right) .
\end{aligned}
$$

Using the notations $R_{11}=R, R_{22}=R_{2}$, and $R_{33}=R_{3}$, the commutative diagram (32) becomes the following commutative diagram

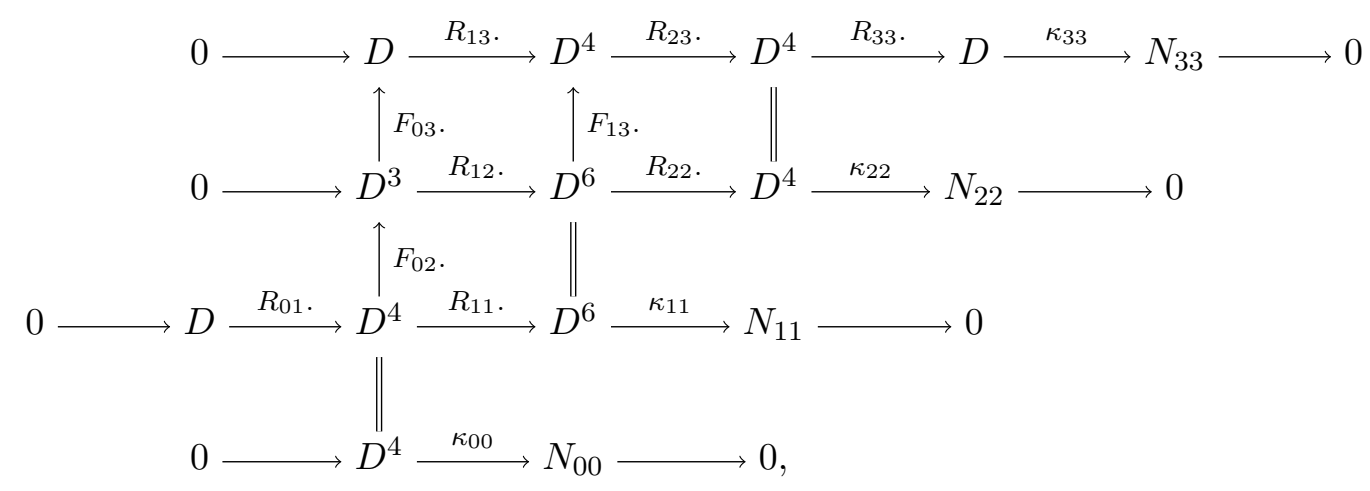

whose horizontal sequences are exact and with the following notations:

$$
\begin{gathered}
R_{01}=\left(\begin{array}{c}
1 \\
-1 \\
1 \\
\partial_{1}-2 \partial_{2}+\partial_{3}
\end{array}\right), R_{12}=\left(\begin{array}{cccc}
1 & 0 & 0 \\
-1 & 4 \partial_{1}-\partial_{3} & 0 \\
1 & 4 \partial_{1}-\partial_{3} & \partial_{3} \\
0 & \partial_{1}-\partial_{2} & 0 \\
0 & \partial_{1}-\partial_{2} & 0 \\
0 & 0 & \partial_{1}
\end{array}\right), \quad R_{23}=\left(\begin{array}{cccc}
-\partial_{3} & \partial_{2} & 0 & 0 \\
0 & 0 & 1 & 0 \\
0 & \partial_{1} & -1 & \partial_{3} \\
\partial_{1} & 0 & 0 & \partial_{2}
\end{array}\right) \\
R_{13}=\left(\begin{array}{c}
-\partial_{2} \\
-\partial_{3} \\
0 \\
\partial_{1}
\end{array}\right), F_{02}=\left(\begin{array}{cccc}
0 & -2 \partial_{1} & -\partial_{1}-2 \partial_{2}+\partial_{3} & -1 \\
0 & -1 & -1 & 0 \\
1 & -1 & -2 & 0
\end{array}\right),
\end{gathered}
$$




$$
F_{13}=\left(\begin{array}{cccccc}
0 & 0 & 0 & 1 & -1 & 0 \\
2 & 1 & -1 & 0 & 0 & 0 \\
2 \partial_{1} & \partial_{2} & -2 \partial_{1}+\partial_{2} & -\partial_{3} & 8 \partial_{1}-\partial_{3} & -8 \partial_{2}+2 \partial_{3} \\
0 & 0 & 0 & 0 & 0 & 1
\end{array}\right), \quad F_{03}=\left(\begin{array}{lll}
0 & 0 & 1
\end{array}\right)
$$

$R_{03}=0$, and $R_{02}=0$. Using Remark 14 with $p_{03}=1$ and $p_{02}=3$, we get $R_{13}^{\prime}=1, R_{12}^{\prime}=I_{3}$, $R_{21}^{\prime}=0, R_{22}^{\prime}=0$, and $R_{23}^{\prime}=0$. Then, 69 becomes the following the commutative diagram

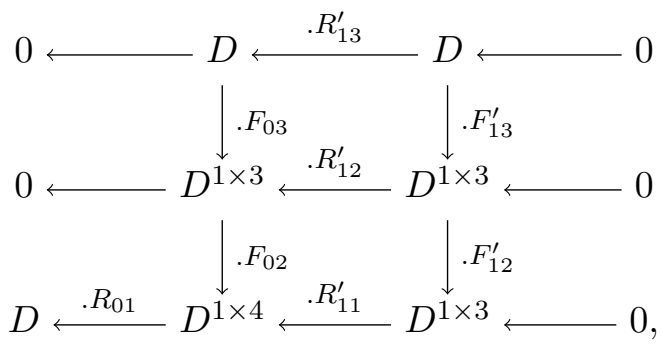

with the following notations:

$$
R_{11}^{\prime}=\left(\begin{array}{cccc}
1 & 0 & -1 & 0 \\
0 & 1 & 1 & 0 \\
0 & 0 & \partial_{1}-2 \partial_{2}+\partial_{3} & -1
\end{array}\right), \quad F_{13}^{\prime}=F_{03}, \quad F_{12}^{\prime}=\left(\begin{array}{ccc}
0 & -2 \partial_{1} & 1 \\
0 & -1 & 0 \\
1 & -1 & 0
\end{array}\right) .
$$

Moreover, using (63), we have $R_{13}^{\prime \prime}=R_{13}, R_{12}^{\prime \prime}=R_{12}$, and:

$$
R_{11}^{\prime \prime}=\left(\begin{array}{ccc}
0 & -2 \partial_{1} & 1 \\
0 & -2 \partial_{1}+\partial_{3} & -1 \\
\partial_{3} & -6 \partial_{1} & 1 \\
0 & -\partial_{1}+\partial_{2} & 0 \\
\partial_{2} & -\partial_{1} & 0 \\
\partial_{1} & -\partial_{1} & 0
\end{array}\right) .
$$

Since $\operatorname{ker}_{D}\left(. R_{3}\right)=0, N_{33} \cong \operatorname{ext}_{D}^{3}(M, D)$ and thus $\operatorname{ext}_{D}^{3}\left(N_{33}, D\right) \cong \operatorname{ext}_{D}^{3}\left(\operatorname{ext}_{D}^{3}(M, D), D\right)$, which shows that $\left\{M_{i}\right\}_{i=0, \ldots, 3}$ defined by (57) is the grade filtration of $M$.

Using (45) and (64) with $N_{11}=D^{6} /\left(R_{11} D^{4}\right), N_{22}=D^{4} /\left(R_{22} D^{6}\right)$, and $N_{33}=D /\left(R_{33} D^{4}\right)$, we obtain the finitely left $D$-modules:

$$
\left\{\begin{array}{l}
L_{1}=D^{1 \times 3} /\left(D^{1 \times 6} R_{11}^{\prime \prime}\right) \cong \operatorname{ext}_{D}^{1}\left(N_{11}, D\right) \cong t(M) \\
L_{2}=D^{1 \times 3} /\left(D^{1 \times 6} R_{12}\right) \cong \operatorname{ext}_{D}^{2}\left(N_{22}, D\right) \\
L_{3}=D /\left(D^{1 \times 4} R_{13}\right) \cong \operatorname{ext}_{D}^{3}\left(N_{33}, D\right)
\end{array}\right.
$$


Corollary 5 yields $M \cong E=D^{1 \times 11} /\left(D^{1 \times 17} Q\right)$, where the matrix $Q$ is defined by:

$$
Q=\left(\begin{array}{ccccccccccc}
1 & 0 & -1 & 0 & -1 & 0 & 0 & 0 & 0 & 0 & 0 \\
0 & 1 & 1 & 0 & 0 & -1 & 0 & 0 & 0 & 0 & 0 \\
0 & 0 & \partial_{1}-2 \partial_{2}+\partial_{3} & -1 & 0 & 0 & -1 & 0 & 0 & 0 & 0 \\
0 & 0 & 0 & 0 & 0 & -2 \partial_{1} & 1 & -1 & 0 & 0 & 0 \\
0 & 0 & 0 & 0 & 0 & -1 & 0 & 0 & -1 & 0 & 0 \\
0 & 0 & 0 & 0 & 1 & -1 & 0 & 0 & 0 & -1 & 0 \\
0 & 0 & 0 & 0 & 0 & 0 & 0 & 0 & 0 & 1 & -1 \\
0 & 0 & 0 & 0 & 0 & 0 & 0 & 1 & 0 & 0 & 0 \\
0 & 0 & 0 & 0 & 0 & 0 & 0 & -1 & 4 \partial_{1}-\partial_{3} & 0 & 0 \\
0 & 0 & 0 & 0 & 0 & 0 & 0 & 1 & 4 \partial_{1}-\partial_{3} & \partial_{3} & 0 \\
0 & 0 & 0 & 0 & 0 & 0 & 0 & 0 & \partial_{1}-\partial_{2} & 0 & 0 \\
0 & 0 & 0 & 0 & 0 & 0 & 0 & 0 & \partial_{1}-\partial_{2} & 0 & 0 \\
0 & 0 & 0 & 0 & 0 & 0 & 0 & 0 & 0 & \partial_{1} & 0 \\
0 & 0 & 0 & 0 & 0 & 0 & 0 & 0 & 0 & 0 & -\partial_{2} \\
0 & 0 & 0 & 0 & 0 & 0 & 0 & 0 & 0 & 0 & -\partial_{3} \\
0 & 0 & 0 & 0 & 0 & 0 & 0 & 0 & 0 & 0 & 0 \\
0 & 0 & 0 & 0 & 0 & 0 & 0 & 0 & 0 & 0 & \partial_{1}
\end{array}\right) .
$$

Let us explicitly compute $\operatorname{ker}_{\mathcal{F}}(Q$.$) , where \mathcal{F}=C^{\infty}\left(\mathbb{R}^{3}\right)$. We first integrate the last diagonal block of $Q$, i.e., the 0 -dimensional (holonomic) linear system $\operatorname{ker}_{\mathcal{F}}\left(R_{13}\right.$.):

$$
\left\{\begin{array}{l}
-\partial_{2} \tau_{3}=0 \\
-\partial_{3} \tau_{3}=0, \\
\partial_{1} \tau_{3}=0
\end{array} \quad \Leftrightarrow \quad \tau_{3}=c_{1} \in \mathbb{R}\right.
$$

Then, we integrate the inhomogeneous linear system in $\tau_{2}=\left(\begin{array}{lll}\tau_{21} & \tau_{22} & \tau_{23}\end{array}\right)^{T}$ and $\tau_{3}$ formed by the third triangular block of $Q$ (whose homogeneous part is purely subholonomic), namely:

$$
\left\{\begin{array} { l } 
{ \tau _ { 2 3 } - \tau _ { 3 } = 0 , } \\
{ \tau _ { 2 1 } = 0 , } \\
{ - \tau _ { 2 1 } + ( 4 \partial _ { 1 } - \partial _ { 3 } ) \tau _ { 2 2 } = 0 , } \\
{ \tau _ { 2 1 } + ( 4 \partial _ { 1 } - \partial _ { 3 } ) \tau _ { 2 2 } + \partial _ { 3 } \tau _ { 2 3 } = 0 , } \\
{ ( \partial _ { 1 } - \partial _ { 2 } ) \tau _ { 2 2 } = 0 , }
\end{array} \Leftrightarrow \left\{\begin{array}{l}
\tau_{23}=\tau_{3}=c_{1}, \\
\tau_{21}=0, \\
\left(4 \partial_{1}-\partial_{3}\right) \tau_{22}=0 \\
\left(\partial_{1}-\partial_{2}\right) \tau_{22}=0 .
\end{array}\right.\right.
$$

We obtain $\tau_{21}=0, \tau_{22}=f_{1}\left(x_{3}+\frac{1}{4}\left(x_{1}+x_{2}\right)\right)$, where $f_{1}$ is an arbitrary smooth function, and $\tau_{23}=c_{1}$, where $c_{1}$ is an arbitrary constant. Then, we integrate the inhomogeneous linear system in $\tau_{1}=\left(\begin{array}{lll}\tau_{11} & \tau_{12} & \tau_{13}\end{array}\right)^{T}$ and $\tau_{2}$ formed by the second triangular block of $Q$, namely:

$$
\left\{\begin{array} { l } 
{ - 2 \partial _ { 1 } \tau _ { 1 2 } + \tau _ { 1 3 } - \tau _ { 2 1 } = 0 , } \\
{ - \tau _ { 1 2 } - \tau _ { 2 2 } = 0 , } \\
{ \tau _ { 1 1 } - \tau _ { 1 2 } - \tau _ { 2 3 } = 0 , }
\end{array} \Leftrightarrow \left\{\begin{array}{l}
\tau_{12}=-\tau_{22}=-f_{1}\left(x_{3}+\frac{1}{4}\left(x_{1}+x_{2}\right)\right), \\
\tau_{11}=-\tau_{22}+\tau_{23}=-f_{1}\left(x_{3}+\frac{1}{4}\left(x_{1}+x_{2}\right)\right)+c_{1}, \\
\tau_{13}=-2 \partial_{1} \tau_{22}+\tau_{21}=-\frac{1}{2} \dot{f}_{1}\left(x_{3}+\frac{1}{4}\left(x_{1}+x_{2}\right)\right) .
\end{array}\right.\right.
$$

The entries of $\tau_{1}$ are 1-dimensional and not 2-dimensional. This result comes from the fact that the matrix $S_{1}^{\prime}$ defined in Remark 16 admits a left inverse over $D$. Thus, we have $M_{1} / M_{2}=0$, 
i.e., $M_{1}=M_{2}$, which yields $\operatorname{ker}_{\mathcal{F}}\left(S_{1}^{\prime}\right.$. $) \cong \operatorname{hom}_{D}\left(\operatorname{coker} \bar{\gamma}_{21}, \mathcal{F}\right) \cong \operatorname{hom}_{D}\left(\operatorname{coker} \gamma_{21}, \mathcal{F}\right)=0$. Finally, we integrate the inhomogeneous linear system in $\zeta=\left(\zeta_{1} \ldots \zeta_{4}\right)^{T}$ and $\tau_{1}$ formed by the first triangular block of $P$, namely:

$$
\left\{\begin{array} { l } 
{ \zeta _ { 1 } - \zeta _ { 3 } - \tau _ { 1 1 } = 0 , } \\
{ \zeta _ { 2 } + \zeta _ { 3 } - \tau _ { 1 2 } = 0 , } \\
{ ( \partial _ { 1 } - 2 \partial _ { 2 } + \partial _ { 3 } ) \zeta _ { 3 } - \zeta _ { 4 } - \tau _ { 1 3 } = 0 , }
\end{array} \Leftrightarrow \left\{\begin{array}{l}
\zeta_{1}-\zeta_{2}=-f_{1}\left(x_{3}+\frac{1}{4}\left(x_{1}+x_{2}\right)\right)+c_{1}, \\
\zeta_{2}+\zeta_{3}=-f_{1}\left(x_{3}+\frac{1}{4}\left(x_{1}+x_{2}\right)\right), \\
\left(\partial_{1}-2 \partial_{2}+\partial_{3}\right) \zeta_{3}-\zeta_{4}=-\frac{1}{2} \dot{f}_{1}\left(x_{3}+\frac{1}{4}\left(x_{1}+x_{2}\right)\right) .
\end{array}\right.\right.
$$

The torsion-free $D$-module $M / t(M)=D^{1 \times 4} /\left(D^{1 \times 3} R_{11}^{\prime}\right)$ can be parametrized by means of $R_{01}$, i.e., $M / t(M) \cong D^{1 \times 4} R_{01}$. Since $\mathcal{F}$ is an injective $D$-module, the linear system $\operatorname{ker}_{\mathcal{F}}\left(R_{11}^{\prime}\right.$.) is parametrized by $R_{01}$, i.e., $\operatorname{ker}_{\mathcal{F}}\left(R_{11}^{\prime}.\right)=R_{01} \mathcal{F}$. Since $R_{11}^{\prime}$ admits the right inverse over $D$

$$
X=\left(\begin{array}{ccc}
1 & 0 & 0 \\
0 & 1 & 0 \\
0 & 0 & 0 \\
0 & 0 & -1
\end{array}\right)
$$

the Quillen-Suslin theorem (see, e.g., [21, 50]) implies that $M / t(M)$ is a free $D$-module of rank 1. The general $\mathcal{F}$-solution of $(89)$ is then defined by $\zeta=R_{01} \xi+X \tau_{1}$ (for more details, see [46]):

$\forall \xi \in C^{\infty}\left(\mathbb{R}^{3}\right), \quad \forall f_{1} \in C^{\infty}(\mathbb{R}), \quad \forall c_{1} \in \mathbb{R}, \quad\left\{\begin{array}{l}\zeta_{1}=\xi-f_{1}\left(x_{3}+\frac{1}{4}\left(x_{1}+x_{2}\right)\right)+c_{1}, \\ \zeta_{2}=-\xi-f_{1}\left(x_{3}+\frac{1}{4}\left(x_{1}+x_{2}\right)\right), \\ \zeta_{3}=\xi, \\ \zeta_{4}=\left(\partial_{1}-2 \partial_{2}+\partial_{3}\right) \xi+\frac{1}{2} \dot{f}_{1}\left(x_{3}+\frac{1}{4}\left(x_{1}+x_{2}\right)\right) .\end{array}\right.$

Finally, using the $D$-isomorphism $\gamma$ defined by (88), we obtain

$$
\left\{\begin{array} { l } 
{ - 2 \partial _ { 1 } \eta _ { 2 } + \partial _ { 3 } \eta _ { 3 } - 2 \partial _ { 2 } \eta _ { 3 } - \partial _ { 1 } \eta _ { 3 } - \eta _ { 4 } = 0 , } \\
{ \partial _ { 3 } \eta _ { 2 } - 2 \partial _ { 1 } \eta _ { 2 } + 2 \partial _ { 2 } \eta _ { 3 } - 3 \partial _ { 1 } \eta _ { 3 } + \eta _ { 4 } = 0 , } \\
{ \partial _ { 3 } \eta _ { 1 } - 6 \partial _ { 1 } \eta _ { 2 } - 2 \partial _ { 2 } \eta _ { 3 } - 5 \partial _ { 1 } \eta _ { 3 } - \eta _ { 4 } = 0 , } \\
{ \partial _ { 2 } \eta _ { 2 } - \partial _ { 1 } \eta _ { 2 } + \partial _ { 2 } \eta _ { 3 } - \partial _ { 1 } \eta _ { 3 } = 0 , } \\
{ \partial _ { 2 } \eta _ { 1 } - \partial _ { 1 } \eta _ { 2 } - \partial _ { 2 } \eta _ { 3 } - \partial _ { 1 } \eta _ { 3 } = 0 , } \\
{ \partial _ { 1 } \eta _ { 1 } - \partial _ { 1 } \eta _ { 2 } - 2 \partial _ { 1 } \eta _ { 3 } = 0 }
\end{array} \Leftrightarrow \left\{\begin{array}{l}
\eta_{1}=\xi-f_{1}\left(x_{3}+\frac{1}{4}\left(x_{1}+x_{2}\right)\right)+c_{1} \\
\eta_{2}=-\xi-f_{1}\left(x_{3}+\frac{1}{4}\left(x_{1}+x_{2}\right)\right) \\
\eta_{3}=\xi \\
\eta_{4}=\left(\partial_{1}-2 \partial_{2}+\partial_{3}\right) \xi+\frac{1}{2} \dot{f}_{1}\left(x_{3}+\frac{1}{4}\left(x_{1}+x_{2}\right)\right)
\end{array}\right.\right.
$$

where $\xi$ (resp., $\left.f_{1}, c_{1}\right)$ is an arbitrary function of $C^{\infty}\left(\mathbb{R}^{3}\right)$ (resp., $C^{\infty}(\mathbb{R})$, constant).

For more examples coming from mathematical physics, mathematical systems theory, and algebraic geometry, see [45]. For instance, using the PURITYFiltration package, we can show that the torsion submodule of the differential module $M$ defined by the linearized Einstein equations in the vacuum (see, e.g., [14]) is 1-pure (see [45]), and thus every nontrivial torsion element $m$ of $M$ defines a pure differential module of dimension 3 .

Using the regular patterns of the matrix $P$ and $(85)$, we can easily generalize Theorem 11 , Corollary 6 and Remark 16 as follows. 
Theorem 12. Let $D$ be a noetherian regular ring $D$ satisfying (38), $\operatorname{gld}(D)=n$, and $R \in D^{q \times p}$. Then, there exists a matrix $\bar{R} \in D^{\bar{q} \times \bar{p}}$ of the form

$$
\bar{R}=\left(\begin{array}{cccccc}
R_{11}^{\prime} & -I_{p_{11}^{\prime}} & 0 & 0 & 0 & 0 \\
0 & F_{12}^{\prime} & -I_{p_{12}^{\prime}} & 0 & 0 & 0 \\
0 & R_{11}^{\prime \prime} & 0 & 0 & 0 & 0 \\
0 & R_{21}^{\prime} & 0 & 0 & 0 & 0 \\
0 & 0 & \vdots & \vdots & 0 & 0 \\
0 & 0 & \vdots & \vdots & 0 & 0 \\
0 & 0 & 0 & 0 & F_{1(n-1)}^{\prime} & -I_{p_{1(n-1)}^{\prime}} \\
0 & 0 & 0 & 0 & R_{1(n-1)}^{\prime \prime} & 0 \\
0 & 0 & 0 & 0 & R_{2(n-1)}^{\prime} & 0 \\
0 & 0 & 0 & 0 & 0 & R_{1 n}^{\prime \prime} \\
0 & 0 & 0 & 0 & 0 & R_{2 n}^{\prime}
\end{array}\right)
$$

such that $M=D^{1 \times p} /\left(D^{1 \times q} R\right) \cong \bar{M}=D^{1 \times \bar{p}} /\left(D^{1 \times \bar{q}} \bar{R}\right)$. Moreover, if $\bar{\pi}: D^{1 \times \bar{p}} \longrightarrow \bar{M}$ is the canonical projection onto $\bar{M}$ and $R_{11}^{\prime} \in D^{p_{11}^{\prime} \times p_{01}}$, then there exist matrices $F_{1 i}^{\prime}$ for $i=2, \ldots, n$ such that:

$$
\begin{aligned}
& \varphi^{-1}: \bar{M} \quad \longrightarrow \quad M \\
& \varphi: M \longrightarrow \bar{M}
\end{aligned}
$$

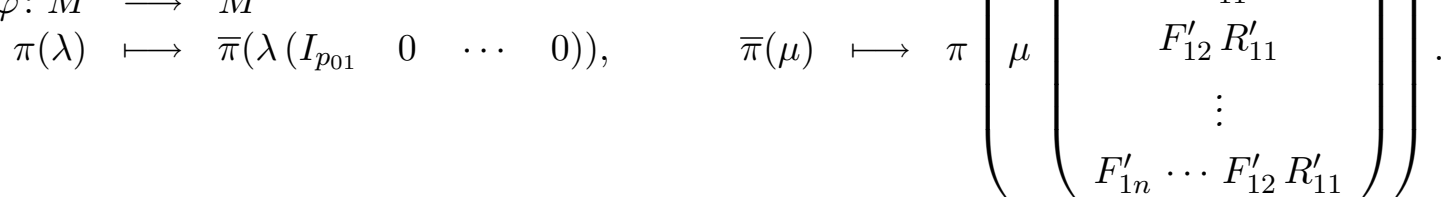

If $\mathcal{F}$ is a left $D$-module, then $\operatorname{ker}_{\mathcal{F}}(R.) \cong \operatorname{ker}_{\mathcal{F}}(\bar{R}$. $)$, where:

$$
\begin{aligned}
& \bar{\gamma}: \operatorname{ker}_{\mathcal{F}}(\bar{R} .) \longrightarrow \operatorname{ker}_{\mathcal{F}}(R .) \quad \bar{\gamma}^{-1}: \operatorname{ker}_{\mathcal{F}}(R .) \longrightarrow \operatorname{ker}_{\mathcal{F}}(\bar{R} .) \\
& \left(\begin{array}{c}
\zeta \\
\tau_{1} \\
\vdots \\
\tau_{n}
\end{array}\right) \longmapsto \eta=\zeta, \quad \eta \longmapsto\left(\begin{array}{c}
\zeta \\
\tau_{1} \\
\vdots \\
\tau_{n}
\end{array}\right)=\left(\begin{array}{c}
I_{p_{01}} \\
R_{11}^{\prime} \\
\vdots \\
F_{1 n}^{\prime} \cdots F_{12}^{\prime} R_{11}^{\prime}
\end{array}\right) \eta
\end{aligned}
$$

Finally, if $D$ is an Auslander regular ring, then the grade filtration $\left\{M_{i}\right\}_{i=0, \ldots, n}$ of $M$ is defined by the left $D$-module $M_{i}$ finitely presented by $\left(R_{1 i}^{\prime \prime T} \quad R_{2 i}^{\prime T}\right)^{T}$, and $M_{i} / M_{i+1}$ is the $i$-pure left $D$ module finitely presented by $R_{11}^{\prime}$ for $i=0$, by $\left(\begin{array}{ll}F_{1 i}^{\prime T} & R_{1 i}^{\prime \prime T} \\ R_{2 i}^{\prime T}\end{array}\right)^{T}$ for $i=1, \ldots, n-1$, and by $\left(\begin{array}{ll}R_{1 n}^{\prime \prime T} & R_{2 n}^{\prime T}\end{array}\right)^{T}$ for $i=n$.

Remark 17. We note that $M_{i}=M_{i+1}$ iff $S_{i}=\left(\begin{array}{lll}F_{1 i}^{\prime T} & R_{1 i}^{\prime \prime T} & R_{2 i}^{\prime T}\end{array}\right)^{T}$ admits a left inverse over $D$. It shows that the matrix $\bar{R}$ can sometimes be simplified especially if Gröbner/Janet bases can be computed over $D$, since the matrix $S_{i}$ does not generally form a Gröbner/Janet basis. Moreover, elementary operations can also be applied to simplify the matrix $S_{i}$ (see, e.g., Example 6). Using inductively Proposition 6, we can then obtain a simple presentation matrix of $M$ with a 
triangular-block form and whose diagonal blocks present the left $D$-modules $M_{i} / M_{i+1}$ 's when they are nontrivial. Such a procedure is implemented in the PurityFiltration package. For related results, see Appendix A of [2]. Finally, if $D$ is a commutative polynomial ring, then Remark 4 can also be used to check whether or not $M_{i} \cong M_{i} / M_{i+1} \oplus M_{i+1}$, i.e., whether or not the corresponding matrix $\left(\begin{array}{llll}I_{p_{i}^{\prime}}^{T} & 0^{T} & 0^{T}\end{array}\right)^{T}$ can be replaced by the trivial matrix $\left(\begin{array}{lll}0^{T} & 0^{T} & 0^{T}\end{array}\right)^{T}$ (which generally helps the integration of the corresponding linear functional system).

Even if the size of the matrix $\bar{R}$ is larger than the one of $R$, the presentation matrix $\bar{R}$ is more tractable for a fine study of the module properties of the left $D$-module $M \cong \bar{M}$ than $R$, for the study of the structural properties of $\operatorname{ker}_{\mathcal{F}}(R$.), as well as for computing closed-form solutions of $\operatorname{ker}_{\mathcal{F}}(R$.) (when they exist). For instance, overdetermined/underdetermined linear PD systems $\operatorname{ker}_{\mathcal{F}}(R$.), which cannot be directly integrated by means of standard computer algebra systems such as Maple, can be done using their equivalent forms $\operatorname{ker}_{\mathcal{F}}(\bar{R}$.). See Appendix and [45].

\section{$5 \quad$ An embedding theorem}

If $D$ is a domain, then a torsion-free left $D$-module $M$ can be embedded into a free left $D$ module (see the comment after Proposition 4 4 , and thus into a projective left $D$-module. Using Example 4, we deduce that a 0 -pure left $D$-module $M$ can be embedded into a left $D$-module of projective dimension 0 . This result is a particular case of the following general result.

Proposition 11 ([10]). Let $D$ be an Auslander regular ring and $M$ an i-pure left D-module. Then, $M$ can be embedded into a left $D$-module $P_{i}$ of left projective dimension $i$, i.e., there exist a left $D$-module $P_{i}$ with $\operatorname{lpd}_{D}\left(P_{i}\right)=i$ and an injective homomorphism $\epsilon_{i} \in \operatorname{hom}_{D}\left(M, P_{i}\right)$.

Proof. Let us give a constructive proof of the result. Let us first prove the result for a 0 pure module $M=D^{1 \times p} /\left(D^{1 \times q} R\right)$, i.e., $t_{0}(M)=M$ and $t_{1}(M)=0$. Since $j_{D}(M)=0$, $\operatorname{ker}_{D}(R.) \cong \operatorname{hom}_{D}(M, D) \neq 0$ (see Theorem 1), which shows that the Auslander transpose $N_{11}=D^{p_{11}} /\left(R_{11} D^{p_{01}}\right)$ of $M=D^{1 \times p_{01}} /\left(D^{1 \times p_{11}} R_{11}\right)\left(R_{11}=R, p_{01}=p, p_{11}=q\right)$ admits a free resolution of the form $\ldots \stackrel{R_{-11}}{\longrightarrow} D^{p_{-11}} \stackrel{R_{01}}{\longrightarrow} D^{p_{01}} \stackrel{R_{11}}{\longrightarrow} D^{p_{11}} \stackrel{\kappa_{11}}{\longrightarrow} N_{11} \longrightarrow 0$, where $R_{01} \neq 0$. Since $T_{1}=\operatorname{ext}_{D}^{1}\left(N_{11}, D\right) \cong M_{1}=t_{1}(M)=0$ (see Theorem 10), then we get the exact sequence $D^{1 \times p_{-11}} \stackrel{. R_{01}}{\longleftarrow} D^{1 \times p_{01}} \stackrel{. R_{11}}{\longleftarrow} D^{1 \times p_{11}}$, which yields $M=\operatorname{coker}_{D}\left(. R_{11}\right) \cong \operatorname{im}_{D}\left(. R_{01}\right) \subseteq D^{1 \times p_{-11}}$, where $D^{1 \times p_{-11}}$ is a free left $D$-module, i.e., $\operatorname{lpd}\left(D^{1 \times p_{-11}}\right)=0$.

Let us now suppose that $i \geq 1$. Since $M$ is $i$-pure, $j_{D}(M)=i$. Hence, if (24) is a free resolution of $M$, then $N_{i i}=D^{p_{i i}} /\left(R_{i i} D^{p_{(i-1) i}}\right)$ admits the free resolution (61), where $R_{i i}=R_{i}, p_{i i}=p_{i}$, and $p_{i(i+1)}=p_{i i}$ (see the notations of Section 3). Now, $\operatorname{ext}_{D}^{i}(M, D)=$ $\operatorname{ker}_{D}\left(R_{(i+1)(i+1)} \cdot\right) / \operatorname{im}_{D}\left(R_{i i}.\right)=\left(R_{i(i+1)} D^{p_{(i-1)(i+1)}}\right) /\left(R_{i i} D^{p_{(i-1) i}}\right)$ is a left $D$-submodule of the left $D$-module $N_{i i}$. Using Proposition 4, we obtain

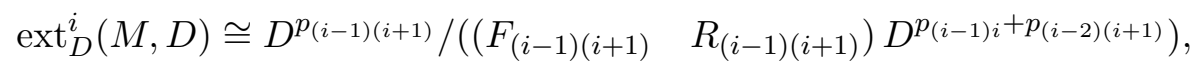

and the following commutative exact diagram holds: 


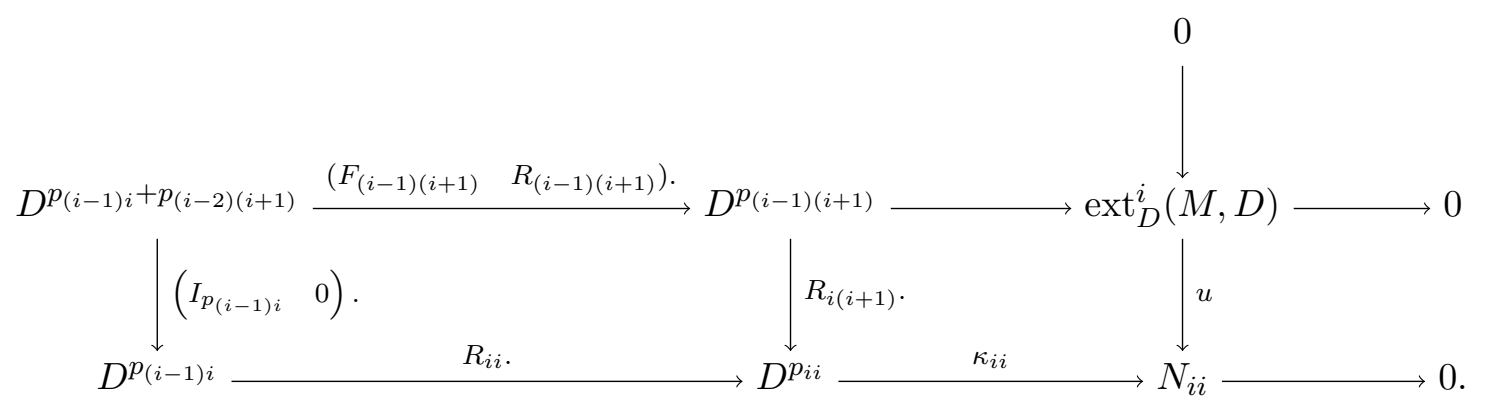

Let $q_{0}=p_{(i-1)(i+1)}, q_{1}=p_{(i-1) i}+p_{(i-2)(i+1)}, Q_{1}=\left(F_{(i-1)(i+1)} \quad R_{(i-1)(i+1)}\right), L_{0}=R_{i(i+1)}$, and $L_{1}=\left(\begin{array}{ll}I_{p_{(i-1) i}} & 0\end{array}\right)$. Extending the free resolution of $\operatorname{ext}_{D}^{i}(M, D), u \in \operatorname{hom}_{D}\left(\operatorname{ext}_{D}^{i}(M, D), N_{i i}\right)$ then induces the following commutative exact diagram:

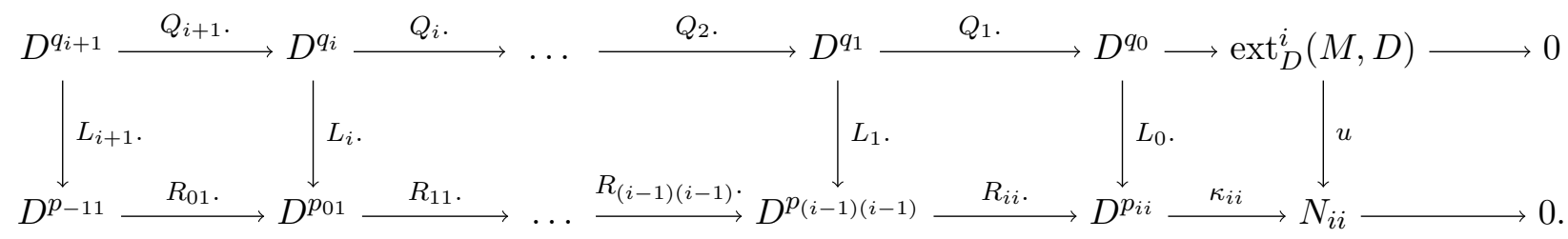

Since $j_{D}(M)=i \geq 1$, Theorem 1 shows that $\operatorname{ker}_{D}\left(R_{1 i}.\right) \cong \operatorname{hom}_{D}(M, D)=0$, i.e., $R_{01}=0$ (see also Remark 13). Since $D$ is Auslander regular (see Remark 7), $\operatorname{hom}_{D}\left(\operatorname{ext}_{D}^{i}(M, D), D\right)=0$ for $i \geq 1$. Applying the contravariant left exact functor $\operatorname{hom}_{D}(\cdot, D)$ to the above commutative exact diagram, we get the following commutative diagram:

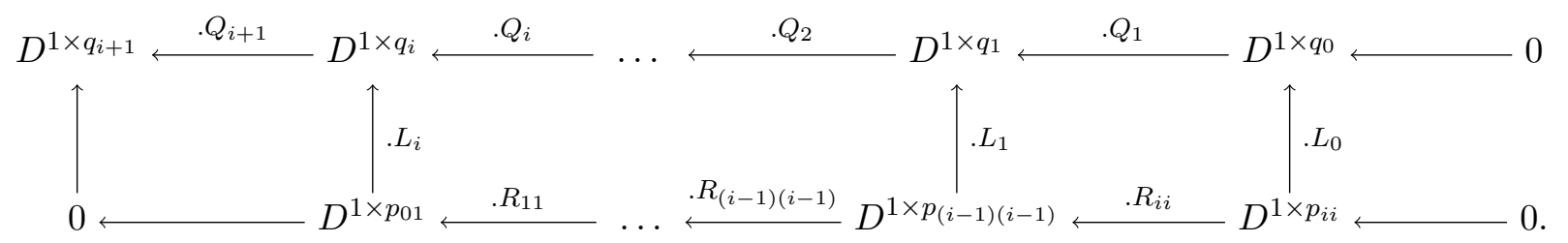

Since $D$ is Auslander regular, $\operatorname{ext}_{D}^{j}\left(\operatorname{ext}_{D}^{i}(M, D), D\right)=0$ for $j=1, \ldots, i-1$, which shows that the top horizontal complex of $(92)$ is exact at $D^{1 \times q_{j}}$ for $j=0, \ldots, i-1$. The defect of exactness of the top horizontal complex at $D^{1 \times q_{i}}$ is $\operatorname{ext}_{D}^{i}\left(\operatorname{ext}_{D}^{i}(M, D), D\right) \cong \operatorname{ker}_{D}\left(. Q_{i+1}\right) / \operatorname{im}_{D}\left(. Q_{i}\right)$, and the defect of exactness of the bottom horizontal complex at $D^{1 \times p_{01}}$ is $\operatorname{ext}_{D}^{i}\left(N_{i i}, D\right) \cong$ $D^{1 \times p_{01}} /\left(D^{1 \times p_{11}} R_{11}\right)=M$. Hence, . $L_{i}$ induces the following canonical left $D$-homomorphism

$$
\begin{aligned}
\varepsilon_{i}: M & \longrightarrow \operatorname{ker}_{D}\left(\cdot Q_{i+1}\right) / \operatorname{im}_{D}\left(. Q_{i}\right) \cong \operatorname{ext}_{D}^{i}\left(\operatorname{ext}_{D}^{i}(M, D), D\right) \\
\pi(\lambda) & \longmapsto o\left(\lambda L_{i}\right),
\end{aligned}
$$

where $o$ : $\operatorname{ker}_{D}\left(. Q_{i+1}\right) \longrightarrow \operatorname{ker}_{D}\left(. Q_{i+1}\right) / \operatorname{im}_{D}\left(. Q_{i}\right)$ is the projection, and $\lambda \in D^{1 \times p_{01}}$. Since $M$ is $i$-pure, 1 of Theorem 8 implies that $\varepsilon_{i}$ is an injective left $D$-homomorphism.

The exactness of the top horizontal complex of 92 at $D^{1 \times q_{j}}$ for $j=0, \ldots, i-1$ shows that the left $D$-module $P_{i}=D^{1 \times q_{i}} /\left(D^{1 \times q_{i-1}} Q_{i}\right)$ admits a free resolution of length $i$, which implies that $\operatorname{ext}_{D}^{j}\left(P_{i}, D\right)=0$ for all $j>i$. The free resolution of $\operatorname{ext}_{D}^{i}(M, D)$ defined by $(92)$ shows that 
$\operatorname{ext}_{D}^{i}\left(P_{i}, D\right) \cong \operatorname{ext}_{D}^{i}(M, D) \neq 0$, which proves that $\operatorname{lpd}_{D}\left(P_{i}\right)=i$ by Proposition 2 . Finally, since $\operatorname{ker}_{D}\left(. Q_{i+1}\right) \subseteq D^{1 \times q_{i}}, \operatorname{ker}_{D}\left(. Q_{i+1}\right) / \operatorname{im}_{D}\left(. Q_{i}\right)$ is a left $D$-submodule of $P_{i}=D^{1 \times q_{i}} /\left(D^{1 \times q_{i-1}} Q_{i}\right)$, $\varepsilon_{i}$ induces an injective left $D$-homomorphism $\epsilon_{i}: M \longrightarrow P_{i}$ defined by $\epsilon_{i}(\pi(\lambda))=\sigma_{i}\left(\lambda L_{i}\right)$ for all $\lambda \in D^{1 \times p_{01}}$, where $\sigma_{i}: D^{1 \times q_{i}} \longrightarrow P_{i}$ is the canonical projection onto $P_{i}$.

The constructive proof of Proposition 11 is implemented in the PURITYFiltration package.

A proof of Proposition 11 based on Spencer cohomology [51] was recently obtained in [39].

Example 7. Let $D$ be an Auslander regular ring with $\operatorname{gld}(D)=n$ and $M$ a nonzero holonomic left $D$-module. In particular, $\operatorname{pd}_{D}(M) \leq n$. By definition of a holonomic module, $j_{D}(M)=n$, and thus $\operatorname{ext}_{D}^{n}(M, D) \neq 0$ and $\operatorname{ext}_{D}^{i}(M, D)=0$ for $i>n$, which proves that $\operatorname{lpd}_{D}(M)=n$ by Proposition 2. Since $M$ is $n$-pure, we can take $P_{n}=M$ and $\epsilon_{n}=\mathrm{id}_{M}$ in Proposition 11.

Example 8. Let $D$ be an Auslander regular ring and $M \neq 0$ a left $D$-module defined by the free resolution $0 \longrightarrow D^{1 \times p} \stackrel{. R}{\longrightarrow} D^{1 \times p} \stackrel{\pi}{\longrightarrow} M \longrightarrow 0$. Since $M \cong \operatorname{ext}_{D}^{1}\left(\operatorname{ext}_{D}^{1}(M, D), D\right)$, i.e., $M$ is 1-pure, and $\operatorname{lpd}_{D}(M)=1$, we can then take $P_{1}=M$ and $\epsilon_{1}=\operatorname{id}_{M}$ in Proposition 11. If $D$ is also a Cohen-Macaulay ring, then $\operatorname{dim}_{D}(M)=\operatorname{dim}(D)-1$. If $D$ is the ring of PD operators with coefficients in a differential field $K$ of characteristic 0 , then this result proves Janet's conjecture [26], which was first obtained by Johnson in [28] (see also [40, 41]).

Corollary 7. Let $D$ be an Auslander regular ring, $M=D^{1 \times p} /\left(D^{1 \times q} R\right)$ an i-pure left $D$-module, and $\mathcal{F}$ an injective left $D$-module. Then, there exist two matrices $Q \in D^{s \times r}$ and $L \in D^{p \times r}$ such that the left $D$-module $P=D^{1 \times r} /\left(D^{1 \times s} Q\right)$ is such that $\operatorname{lpd}_{D}(P)=i$, and

$$
\operatorname{ker}_{\mathcal{F}}(R .)=L \operatorname{ker}_{\mathcal{F}}(Q .),
$$

i.e., an i-pure linear system is the image of a linear system of projective dimension $i$.

Proof. The proof of Proposition 11 shows that the following commutative exact diagram holds

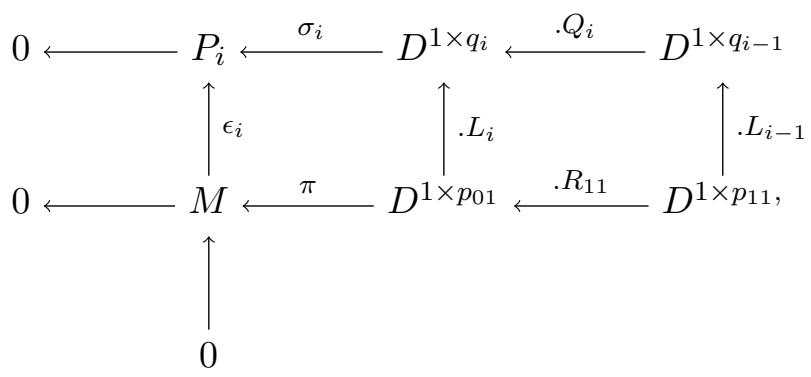

where $R_{11}=R, p_{01}=p$, and $p_{11}=q$. Applying the contravariant exact functor $\operatorname{hom}_{D}(\cdot, \mathcal{F})$ to (93), we obtain the following commutative exact diagram

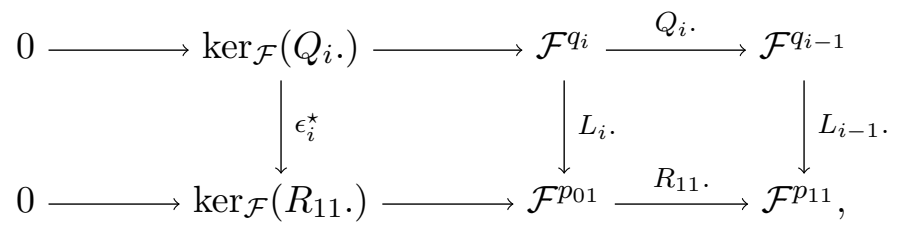

which shows that $\epsilon_{i}^{\star}: \operatorname{ker}_{\mathcal{F}}\left(Q_{i}\right.$. $) \longrightarrow \operatorname{ker}_{\mathcal{F}}(R$. $)$ is defined by $\epsilon_{i}^{\star}(\xi)=L_{i} \xi$ for all $\xi \in \operatorname{ker}_{\mathcal{F}}\left(Q_{i}.\right)$. Using Theorem 3 , the short exact sequence $0 \longrightarrow M \stackrel{\epsilon_{i}}{\longrightarrow} P_{i} \longrightarrow$ coker $\epsilon_{i} \longrightarrow 0$ yields the long exact sequence $0 \longrightarrow \operatorname{hom}_{D}\left(\operatorname{coker} \epsilon_{i}, \mathcal{F}\right) \longrightarrow \operatorname{hom}_{D}\left(P_{i}, \mathcal{F}\right) \longrightarrow \operatorname{hom}_{D}(M, \mathcal{F}) \longrightarrow \operatorname{ext}_{D}^{1}\left(\operatorname{coker} \epsilon_{i}, \mathcal{F}\right)$. 
Since $\mathcal{F}$ is an injective left $D$-module, $\operatorname{ext}_{D}^{1}\left(\operatorname{coker} \epsilon_{i}, \mathcal{F}\right)=0$ (see Definition 3), which shows that $\epsilon_{i}^{\star}$ is surjective, i.e., using Theorem 11, for every $\eta \in \operatorname{ker}_{\mathcal{F}}(R$. $)$, there exists $\xi \in \operatorname{ker}_{\mathcal{F}}\left(Q_{i}\right.$. ) such that $\eta=L_{i} \xi$. We note that $\epsilon_{i}^{\star}$ is also injective iff $\operatorname{hom}_{D}\left(\operatorname{coker} \epsilon_{i}, \mathcal{F}\right) \cong \operatorname{ker}_{\mathcal{F}}\left(\left(\begin{array}{ll}L_{i}^{T} & Q_{i}\end{array}\right)^{T}\right.$.) $=0$.

Example 9. Let $M$ be the $D=\mathbb{Q}\left[\partial_{1}, \partial_{2}, \partial_{3}\right]$-module finitely presented by the following matrix:

$$
R=\left(\begin{array}{cc}
\partial_{1} & 0 \\
0 & \partial_{1} \\
\partial_{2} & -\partial_{3}
\end{array}\right) \in D^{3 \times 2}
$$

Then, the $D$-module $M$ admits the following free resolution:

$$
0 \longleftarrow M \stackrel{\pi}{\longleftarrow} D^{1 \times 2} \stackrel{. R}{\longleftarrow} D^{1 \times 3} \stackrel{. R_{2}}{\longleftarrow} D \longleftarrow 0, \quad R_{2}=\left(\begin{array}{lll}
-\partial_{2} & \partial_{3} & \partial_{1}
\end{array}\right) .
$$

Clearly, $\operatorname{ext}_{D}^{2}(M, D)=D /\left(\partial_{1}, \partial_{2}, \partial_{3}\right) \neq 0$, which shows that $\operatorname{pd}_{D}(M)=2$ by Proposition 2. Using Algorithm 1, we can check that $M=M_{1}=t(M)$ and $M_{2} \cong \operatorname{ext}_{D}^{2}\left(N_{22}, D\right)=0$, where $N_{22}=D /\left(\partial_{1}, \partial_{2}, \partial_{3}\right)$, which shows that $M$ is a 1-pure $D$-module. With the notations of Section 3 and of the proof of Proposition 11, i.e., $R_{11}=R, R_{22}=R_{2}, \operatorname{ker}_{D}\left(R_{22}.\right)=R_{12} D^{3}, \operatorname{ker}_{D}\left(R_{12}.\right)=$ $R_{02} D, R_{12} F_{02}=R_{11}, Q_{1}=\left(\begin{array}{ll}F_{02} & R_{02}\end{array}\right), L_{0}=R_{12}$, and $L_{1}=\left(\begin{array}{ll}I_{2} & 0\end{array}\right)$, where

$$
R_{12}=\left(\begin{array}{ccc}
\partial_{3} & \partial_{1} & 0 \\
\partial_{2} & 0 & \partial_{1} \\
0 & \partial_{2} & -\partial_{3}
\end{array}\right), \quad F_{02}=\left(\begin{array}{cc}
0 & 0 \\
1 & 0 \\
0 & 1
\end{array}\right), \quad R_{02}=\left(\begin{array}{c}
-\partial_{1} \\
\partial_{3} \\
\partial_{2}
\end{array}\right),
$$

we obtain $\operatorname{ext}_{D}^{1}(M, D)=\operatorname{ker}_{D}\left(R_{22}.\right) /\left(R_{11} D^{2}\right)=\left(R_{12} D^{3}\right) /\left(R_{11} D^{2}\right) \cong D^{3} /\left(Q_{1} D^{3}\right)$. By Proposition 11, the $D$-homomorphism $\epsilon: M \longrightarrow P_{1}=D^{1 \times 3} /\left(D^{1 \times 3} Q_{1}\right)$ defined by $\epsilon_{1}(\pi(\lambda))=\sigma_{1}\left(\lambda L_{1}\right)$ is injective. Since the matrix $Q_{1}$ has full row rank and $P_{1} \neq 0, \operatorname{pd}_{D}\left(P_{1}\right)=1$, which shows that the 1-pure $D$-module $M$ can be embedded into the $D$-module $P_{1}$ of projective dimension 1 . Finally, if $\mathcal{F}=C^{\infty}\left(\mathbb{R}^{3}\right)$ is the injective $D$-module of smooth functions (see Example 2), then

$$
\operatorname{ker}_{\mathcal{F}}\left(Q_{1} .\right)=\left\{\left(\partial_{3} \phi\left(x_{2}, x_{3}\right) \quad \partial_{2} \phi\left(x_{2}, x_{3}\right) \quad-\phi\left(x_{2}, x_{3}\right)\right)^{T} \mid \forall \phi \in C^{\infty}\left(\mathbb{R}^{2}\right)\right\},
$$

which gives $\operatorname{ker}_{\mathcal{F}}(R)=.L_{1} \operatorname{ker}_{\mathcal{F}}\left(Q_{1}.\right)=\left\{\left(\partial_{3} \phi\left(x_{2}, x_{3}\right) \quad \partial_{2} \phi\left(x_{2}, x_{3}\right)\right)^{T} \mid \forall \phi \in C^{\infty}\left(\mathbb{R}^{2}\right)\right\}$.

\section{Acknowledgements}

We are grateful to M. Barakat (University of Kaiserslautern) and J.-F. Pommaret (Ecole Nationale des Ponts et Chaussées) for stimulating discussions on grade filtration. We also would like to thank D. Robertz (RWTH Aachen University) and G. Regensburger (INRIA Saclay-Îlede-France) for their comments on the literary aspect of some parts of the paper.

\section{Appendix: The PurityFiltration package}

We demonstrate the PurityFiltration package (Maple 15) dedicated to grade filtration and its applications. It uses the OreModules package [15] and the OreMorPhISMS package [17.

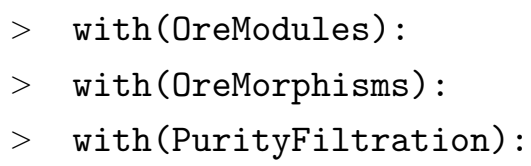

Since the notation $D$ is protected in Maple, in what follows, we shall use $A$ instead of $D$. 


\subsection{Grade filtration of linear PD systems}

Example 10. Let $A$ be the ring of PD operators in $d_{1}=\frac{\partial}{\partial x_{1}}$ and $d_{2}=\frac{\partial}{\partial x_{2}}$ with coefficients in $\mathbb{Q}\left[x_{1}, x_{2}\right]$.

$>A:=$ DefineOreAlgebra $(\operatorname{diff}=[d[1], x[1]], \operatorname{diff}=[d[2], x[2]]$, polynom=[x[1], $x[2]]):$

Let us consider the following matrix $R \in A^{3 \times 3}$ of $\mathrm{PD}$ operators first considered by Janet and studied in J.-F. Pommaret, "Algebraic analysis of control systems defined by partial differential equations", Lecture Notes in Control and Inform. Sci., 311, Springer, 2005, pp. 155-223.

$>\quad R:=\operatorname{matrix}(3,3,[0, d[2]-d[1], d[2]-d[1], d[2],-d[1],-d[2]-d[1], d[1],-d[1]$,

$>-2 * \mathrm{~d}[1]])$;

$$
R:=\left[\begin{array}{ccc}
0 & d_{2}-d_{1} & d_{2}-d_{1} \\
d_{2} & -d_{1} & -d_{2}-d_{1} \\
d_{1} & -d_{1} & -2 d_{1}
\end{array}\right]
$$

Let us compute the grade number $j_{A}(M)$ of the $A$-module $M=A^{1 \times 3} /\left(A^{1 \times 3} R\right)$.

$>$ GradeNumber $(\mathrm{R}, \mathrm{A})$;

Let us check that $j_{A}(M)=\operatorname{codim}_{A}(M)$ by computing the codimension of $M$.

$>$ Codimension $(\mathrm{R}, \mathrm{A})$;

Let us check whether or not $M$ is a pure $A$-module.

$>\operatorname{IsPure}(\mathrm{R}, \mathrm{A})$;

$$
\text { false }
$$

Since $M$ is not a pure $A$-module, it admits a nontrivial grade filtration. Let us compute it.

$>\mathrm{G}:=$ GradeFiltrationByGenerators $(\mathrm{R}, \mathrm{A})$;

$$
G:=\left[\left[\left[\begin{array}{ccc}
1 & 0 & -1 \\
0 & 1 & 1
\end{array}\right], 1\right],\left[\left[\begin{array}{ccc}
-1 & 1 & 2
\end{array}\right], 2\right]\right]
$$

We obtain that the $A$-modules $M_{1}=\left(A^{1 \times 2} G_{11}\right) /\left(A^{1 \times 3} R\right)$ and $M_{2}=\left(A G_{21}\right) /\left(A^{1 \times 3} R\right)$ define the grade filtration of $M$, where $G_{i 1}$ is the first matrix of the $i^{\text {th }}$ entry of $G$ (the second entry $G_{i 2}$ is the index $i$ of the submodule $M_{i}$ ). If $\pi: A^{1 \times 3} \longrightarrow M$ is the canonical projection onto $M,\left\{f_{j}\right\}_{j=1,2,3}$ the standard basis of $A^{1 \times 3},\left\{y_{j}=\pi\left(f_{j}\right)\right\}_{j=1,2,3}$ a family of generators of $M$, and $y=\left(\begin{array}{lll}y_{1} & y_{2} & y_{3}\end{array}\right)^{T}$, then $M$ is defined by the relations $R y=0$. Then, we have:

$$
\left\{\begin{array}{l}
M_{0}=M=A y_{1}+A y_{2}+A y_{3} \\
M_{1}=A\left(y_{1}-y_{3}\right)+A\left(y_{2}+y_{3}\right) \\
M_{2}=A\left(-y_{1}+y_{1}+2 y_{3}\right) \\
M_{3}=0
\end{array}\right.
$$

If an option is added to the command GradeFiltrationByGenerators, then we can also obtain the PD equations satisfied by the generators of the $A$-module $M_{i}$ for $i=0,1,2$. The PD 
operators annihilating the $j^{\text {th }}$ generators of $M_{i}$ are the entries of $j^{\text {th }}$ block-diagonal matrix of the matrix in front of the matrix $G_{i 1}$, i.e.,

$>\mathrm{H}:=$ GradeFiltrationByGenerators $(\mathrm{R}, \mathrm{A}, \mathrm{opt})$;

$$
\left.H:=\left[\left[\begin{array}{cc}
-d_{2}+d_{1} & 0 \\
0 & -d_{2}+d_{1}
\end{array}\right],\left[\begin{array}{ccc}
1 & 0 & -1 \\
0 & 1 & 1
\end{array}\right], 1\right],\left[\left[\begin{array}{c}
d_{2} \\
d_{1}
\end{array}\right],\left[\begin{array}{lll}
-1 & 1 & 2
\end{array}\right], 2\right]\right]
$$

shows that $z_{1}=y_{1}-y_{3}$ (resp., $z_{2}=y_{2}+y_{3}$ ) satisfies the PD operators appearing in the first (resp., second) block-diagonal matrix of the matrix appearing in front of $G_{i 1}$, i.e., $\left(d_{1}-d_{2}\right) z_{1}=0$ (resp., $\left(d_{1}-d_{2}\right) z_{2}=0$ ). The generator $z_{3}=-y_{1}+y_{2}+2 y_{3}$ of $M_{2}$ satisfies $d_{2} z_{3}=0$ and $d_{1} z_{3}=0$.

A presentation matrix of the $A$-module $M_{i} / M_{i+1}$ is computed by the command PureFactors_NR:

$>\mathrm{J}:=$ PureFactors_NR $(\mathrm{R}, \mathrm{A})$;

$$
\left.J:=\left[\begin{array}{ccc}
1 & 0 & -1 \\
0 & 1 & 1
\end{array}\right],\left[\begin{array}{cc}
1 & -1 \\
0 & -d_{2}+d_{1}
\end{array}\right],\left[\begin{array}{cc}
1 & 0 \\
0 & d_{1} \\
0 & d_{2}
\end{array}\right]\right]
$$

We get $M / M_{1}=A^{1 \times 3} /\left(A^{1 \times 2} J_{1}\right), M_{1} / M_{2}=A^{1 \times 2} /\left(A^{1 \times 2} J_{2}\right)$, and $M_{2}=A^{1 \times 2} /\left(A^{1 \times 3} J_{3}\right)$, where $J_{i}$ is the $i^{\text {th }}$ matrix of $J$. The suffix _NR stands for "NonReduced", i.e., the matrix $J_{i}$ 's does not generally form a Gröbner basis or is not simplified. To obtain such a presentation matrix of the $A$-module $M_{i} / M_{i+1}$ for $i=0,1,2$, we can use the command PureFactors

$>\mathrm{F}:=$ PureFactors $(\mathrm{R}, \mathrm{A})$;

$$
F:=\left[[0],\left[-d_{2}+d_{1}\right],\left[\begin{array}{l}
d_{1} \\
d_{2}
\end{array}\right]\right]
$$

i.e., we have:

$$
\left\{\begin{array}{l}
M / M_{1} \cong A /\left(A F_{1}\right) \cong A \\
M_{1} / M_{2} \cong A /\left(A F_{2}\right)=A /\left(A\left(d_{1}-d_{2}\right)\right) \\
M_{2} \cong A /\left(A^{1 \times 2} F_{3}\right)=A /\left(A d_{1}+A d_{2}\right)
\end{array}\right.
$$

Let us compute the codimension of the $A$-module $M_{i} / M_{i+1}$ for $i=0,1,2$ :

$>\operatorname{map}($ Codimension, $\mathrm{F}, \mathrm{A})$;

$$
[0,1,2]
$$

Thus, $\operatorname{codim}_{A}\left(M / M_{1}\right)=0, \operatorname{codim}_{A}\left(M_{1} / M_{2}\right)=1$, and $\operatorname{codim}_{A}\left(M_{2}\right)=2$, i.e., $\operatorname{dim}_{A}\left(M / M_{1}\right)=2$, $\operatorname{dim}_{A}\left(M_{1} / M_{2}\right)=1$, and $\operatorname{dim}_{A}\left(M_{2}\right)=0$.

Let us now check that the $A$-module $M_{i} / M_{i+1}$ is $i$-pure for $i=0,1,2$ :

$>\operatorname{map}($ IsPure, $, \mathrm{F}, \mathrm{A})$;

$$
[0,1,2]
$$

Another way to define the grade filtration $\left\{M_{i}\right\}_{i=0, \ldots, 2}$ of $M$ is by means of finitely presented $A$-modules $L_{i} \cong M_{i}$ and injective $\theta_{i} \in \operatorname{hom}_{A}\left(L_{i}, M\right)$ for $i=1,2$ (see Algorithm 3).

$>\mathrm{H}:=$ GradeFiltrationByMorphisms $(\mathrm{R}, \mathrm{A})$; 


$$
\left.H:=\left[\left[\begin{array}{cc}
0 & -d_{2}+d_{1} \\
d_{2} & -d_{2} \\
d_{1} & -d_{2}
\end{array}\right],\left[\begin{array}{ccc}
1 & 0 & -1 \\
0 & 1 & 1
\end{array}\right]\right],\left[\left[\begin{array}{cc}
1 & 0 \\
0 & d_{1} \\
0 & d_{2}
\end{array}\right],\left[\begin{array}{ccc}
0 & d_{2}-d_{1} & d_{2}-d_{1} \\
-1 & 1 & 2
\end{array}\right]\right]\right]
$$

We have $L_{1}=A^{1 \times 2} /\left(A^{1 \times 3} H_{11}\right)$ and $L_{2}=A^{1 \times 2} /\left(A^{1 \times 3} H_{21}\right)$, where $H_{i 1}$ is the first matrix in the $i^{\text {th }}$ entry of $H$. Moreover, the injective $A$-homomorphism $\theta_{i}: L_{i} \longrightarrow M$ is defined by $\theta_{i}\left(\rho_{i}^{\prime}(\lambda)\right)=\pi\left(\lambda H_{i 2}\right)$, where $H_{i 2}$ is the second matrix in the $i^{\text {th }}$ entry of $H$ and $\rho_{i}^{\prime}$ is the canonical projection onto $L_{i}$. Let us check again that the $A$-homomorphisms $\theta_{i}$ 's are injective.

$$
\begin{gathered}
>\operatorname{seq}(\operatorname{TestInj}(H[i][1], R, H[i][2], A), i=1 . .2) ; \\
\text { true, true }
\end{gathered}
$$

Let us now compute an $A$-module $\bar{M}$ isomorphic to $M$ which is finitely presented by the matrix $\bar{R}$ defined by means of the grade filtration of $M$ (see Theorem 12 ).

$>\mathrm{P}:=$ PurePresentation_NR $(\mathrm{R}, \mathrm{A})$;

$$
\begin{gathered}
P:=\left[\begin{array}{ccc}
0 & d_{2}-d_{1} & d_{2}-d_{1} \\
d_{2} & -d_{1} & -d_{2}-d_{1} \\
d_{1} & -d_{1} & -2 d_{1}
\end{array}\right],\left[\begin{array}{ccccccc}
1 & 0 & -1 & -1 & 0 & 0 & 0 \\
0 & 1 & 1 & 0 & -1 & 0 & 0 \\
0 & 0 & 0 & 0 & d_{2}-d_{1} & -1 & 0 \\
0 & 0 & 0 & -1 & 1 & 0 & -1 \\
0 & 0 & 0 & 0 & 0 & 1 & 0 \\
0 & 0 & 0 & 0 & 0 & 1 & -d_{2} \\
0 & 0 & 0 & 0 & 0 & 0 & -d_{1}
\end{array}\right] \text {, } \\
\left.\left[\begin{array}{ccccccc}
1 & 0 & 0 & 0 & 0 & 0 & 0 \\
0 & 1 & 0 & 0 & 0 & 0 & 0 \\
0 & 0 & 1 & 0 & 0 & 0 & 0
\end{array}\right],\left[\begin{array}{cccc}
1 & 0 & 0 \\
0 & 1 & 0 \\
0 & 0 & 1 \\
1 & 0 & -1 \\
0 & 1 & 1 \\
0 & d_{2}-d_{1} & d_{2}-d_{1} \\
-1 & 1 & 2
\end{array}\right]\right]
\end{gathered}
$$

We get $\bar{M}=A^{1 \times 7} /\left(A^{1 \times 7} P_{2}\right) \cong M=A^{1 \times 3} /\left(A^{1 \times 3} P_{1}\right)$, where $P_{i}$ is the $i^{\text {th }}$ matrix of $P$. If $\bar{\pi}$ is the canonical projection onto $\bar{M}$, then $\varphi: M \longrightarrow \bar{M}$ defined by $\varphi(\pi(\lambda))=\bar{\pi}\left(\lambda P_{3}\right)$ is an isomorphism, whose inverse $\varphi^{-1}: \bar{M} \longrightarrow M$ is $\varphi^{-1}(\bar{\pi}(\mu))=\pi\left(\mu P_{4}\right)$.

Let us check that $\varphi$ is an isomorphism and $\varphi^{-1}$ is defined by $P_{4}$.

$$
\begin{aligned}
& >\text { TestIso(P[1], } \mathrm{P}[2], \mathrm{P}[3], \mathrm{A}) \text {; } \\
& \text { true } \\
& >\text { TestIso(P[2], } \mathrm{P}[1], \mathrm{P}[4], \mathrm{A}) \text {; }
\end{aligned}
$$

The matrix $\bar{R}$, defined by the above matrices $J_{i}$ 's, can be simplified by computing a Gröbner basis of the $A$-module defined by the matrix $J_{i}$ for $i=1,2,3$. This can be obtained by using the command PurePresentation: 
$>\mathrm{Q}:=$ PurePresentation $(\mathrm{R}, \mathrm{A})$;

$$
Q:=\left[\left[\begin{array}{ccc}
0 & d_{2}-d_{1} & d_{2}-d_{1} \\
d_{2} & -d_{1} & -d_{2}-d_{1} \\
d_{1} & -d_{1} & -2 d_{1}
\end{array}\right],\left[\begin{array}{ccc}
0 & 0 & 0 \\
0 & -d_{2}+d_{1} & 0 \\
0 & 0 & d_{1} \\
0 & 0 & d_{2}
\end{array}\right],\left[\begin{array}{ccc}
1 & 1 & -1 \\
-1 & 1 & 0 \\
1 & 0 & 0
\end{array}\right],\left[\begin{array}{ccc}
0 & 0 & 1 \\
0 & 1 & 1 \\
-1 & 1 & 2
\end{array}\right]\right]
$$

We obtain $M=A^{1 \times 3} /\left(A^{1 \times 3} Q_{1}\right) \cong L=A^{1 \times 3} /\left(A^{1 \times 3} Q_{2}\right)$, where $Q_{i}$ is the $i^{\text {th }}$ matrix of $Q$. The isomorphism $\psi: M \longrightarrow L$ is defined by $\psi(\pi(\lambda))=\vartheta\left(\lambda Q_{3}\right)$, where $\vartheta$ is the canonical projection onto $L$. Let us check that $\psi$ is an isomorphism.

$$
>\operatorname{TestIso}(\mathrm{Q}[1], \mathrm{Q}[2], \mathrm{Q}[3], \mathrm{A}) \text {; }
$$

\section{true}

Now, $\psi^{-1}: L \longrightarrow M$ is defined by $\psi^{-1}(\vartheta(\mu))=\pi\left(\mu Q_{4}\right)$.

$$
>\text { TestIso(Q[2], Q[1], Q[4], A); }
$$

\section{true}

The presentation matrix $Q_{2}$ of the $A$-module $L$ is defined by the presentation matrices $F_{i}$ 's of the pure $A$-modules $M_{i} / M_{i+1}$ for $i=0,1,2$. The fact that $F_{1}=0$ explains why the first row of $Q_{2}$ is 0 . The presentation matrix $Q_{2}$ can be again simplified using the command SimplifiedPresentation.

$>\mathrm{S}:=$ SimplifiedPresentation $(\mathrm{Q}[2], \mathrm{A})$;

$$
\left.S:=\left[\begin{array}{ccc}
0 & -d_{2}+d_{1} & 0 \\
0 & 0 & d_{1} \\
0 & 0 & d_{2}
\end{array}\right],\left[\begin{array}{ccc}
1 & 0 & 0 \\
0 & 1 & 0 \\
0 & 0 & 1
\end{array}\right],\left[\begin{array}{ccc}
1 & 0 & 0 \\
0 & 1 & 0 \\
0 & 0 & 1 \\
0 & 0 & 0
\end{array}\right]\right]
$$

We have $L=A^{1 \times 3} /\left(A^{1 \times 3} S_{1}\right)$, where $S_{1}$ is the first matrix of $S$ (the second and the third matrices $S_{2}$ and $S_{3}$ defining the identity homomorphism between the two different presentations of $L)$.

Let us compute a presentation of the $A$-module $M_{1}=t(M)$ based on the terms $\left\{M_{i}\right\}_{i=1,2}$ of the grade filtration of $M_{1}$.

$>\mathrm{T}:=$ PurePresentationOfTorsionSubmodule $(\mathrm{R}, \mathrm{A})$;

$$
T:=\left[\left[\begin{array}{cc}
d_{2}-d_{1} & 0 \\
0 & d_{1} \\
0 & d_{2}
\end{array}\right],\left[\begin{array}{ccc}
0 & d_{2}-d_{1} & d_{2}-d_{1} \\
d_{2} & -d_{1} & -d_{2}-d_{1} \\
d_{1} & -d_{1} & -2 d_{1}
\end{array}\right],\left[\begin{array}{ccc}
0 & 1 & 1 \\
-1 & 1 & 2
\end{array}\right]\right]
$$

The first (resp., second) matrix $T_{1}$ (resp., $T_{2}$ ) of $T$ is a presentation of $t(M)$ (resp., $M$ ), i.e., $t(M) \cong K=A^{1 \times 2} /\left(A^{1 \times 3} T_{1}\right)$ (resp., $M=A^{1 \times 3} /\left(A^{1 \times 3} T_{2}\right)$ ). The third matrix $T_{3}$ of $T$ defines the embedding of the $A$-module $K$ into $M$, i.e., defines an injective $\iota \in \operatorname{hom}_{A}(K, M)$ defined by $\iota(\sigma(\nu))=\pi\left(\nu T_{3}\right)$, where $\sigma: A^{1 \times 2} \longrightarrow K$ is the canonical projection onto $K$.

$$
>\operatorname{TestInj}(\mathrm{T}[1], \mathrm{T}[2], \mathrm{T}[3], \mathrm{A}) \text {; }
$$


The form of the matrix $S_{1}$ shows that $L \cong A \oplus K$, and the form of the matrix $T_{1}$ shows that $t(M)=M_{1}=M_{1} / M_{2} \oplus M_{2}$. Thus, we obtain:

$$
M=A \oplus M_{1} / M_{2} \oplus M_{2}=A \oplus A /\left(A\left(d_{2}-d_{1}\right)\right) \oplus A /\left(A d_{1}+A d_{2}\right) .
$$

Let us finally check that $K$ is a torsion $A$-module, i.e., $\operatorname{codim}_{A}(M) \geq 1$.

$>\operatorname{Codimension}(\mathrm{T}[1], \mathrm{A})$;

Example 11. Let $A$ be the ring of PD operators in $d_{1}=\frac{\partial}{\partial x_{1}}, d_{2}=\frac{\partial}{\partial x_{2}}$, and $d_{3}=\frac{\partial}{\partial x_{3}}$ with coefficients in $\mathbb{Q}\left[x_{1}, x_{2}, x_{3}\right]$

$>A:=$ DefineOreAlgebra $(\operatorname{diff}=[\mathrm{d}[1], \mathrm{x}[1]], \operatorname{diff}=[\mathrm{d}[2], \mathrm{x}[2]], \operatorname{diff}=[\mathrm{d}[3], \mathrm{x}[3]]$,

$>$ polynom $=[\mathrm{x}[1], \mathrm{x}[2], \mathrm{x}[3]])$ :

and $R$ the system matrix of the linear PD system defined by the left hand side of (90):

$>\mathrm{R}:=\operatorname{matrix}(6,4,[0,-2 * \mathrm{~d}[1], \mathrm{d}[3]-2 * \mathrm{~d}[2]-\mathrm{d}[1],-1,0, \mathrm{~d}[3]-2 * \mathrm{~d}[1], 2 * \mathrm{~d}[2]-3 * \mathrm{~d}[1]$,

$>1, d[3],-6 * d[1],-2 * d[2]-5 * d[1],-1,0, d[2]-d[1], d[2]-d[1], 0, d[2],-d[1]$,

$>-d[2]-d[1], 0, d[1],-d[1],-2 * d[1], 0])$;

$$
R:=\left[\begin{array}{cccc}
0 & -2 d_{1} & d_{3}-2 d_{2}-d_{1} & -1 \\
0 & d_{3}-2 d_{1} & 2 d_{2}-3 d_{1} & 1 \\
d_{3} & -6 d_{1} & -2 d_{2}-5 d_{1} & -1 \\
0 & d_{2}-d_{1} & d_{2}-d_{1} & 0 \\
d_{2} & -d_{1} & -d_{2}-d_{1} & 0 \\
d_{1} & -d_{1} & -2 d_{1} & 0
\end{array}\right]
$$

Let us study the $A$-module $M=A^{1 \times 4} /\left(A^{1 \times 6} R\right)$. Let us first compute its grade number $j_{A}(M)$.

$>$ GradeNumber $(\mathrm{R}, \mathrm{A})$;

Let us check that $j_{A}(M)=\operatorname{codim}_{A}(M)$ by computing the codimension of $M$.

$>\operatorname{Codimension}(\mathrm{R}, \mathrm{A})$;

0

Let us check whether or not $M$ is a pure $A$-module.

$>\operatorname{IsPure}(\mathrm{R}, \mathrm{A})$;

false

Let us now compute the grade filtration $\{M\}_{i=0, \ldots, 3}$ of $M$ :

$$
\begin{aligned}
& >G:=\operatorname{GradeFiltrationByGenerators~}(\mathrm{R}, \mathrm{A}) \\
& \left.G:=\left[\left[\begin{array}{cccc}
1 & 0 & -1 & 0 \\
0 & 1 & 1 & 0 \\
0 & 0 & -2 d_{2}+d_{3}+d_{1} & -1
\end{array}\right], 1\right],\left[\left[\begin{array}{cccc}
0 & 1 & 1 & 0 \\
-1 & 1 & 2 & 0
\end{array}\right], 2\right],\left[\left[\begin{array}{cccc}
1 & -1 & -2 & 0
\end{array}\right], 3\right]\right]
\end{aligned}
$$


We have $0 \subseteq M_{3} \subseteq M_{2} \subseteq M_{1} \subseteq M_{0}=M$, where

$$
\left\{\begin{array}{l}
M_{0}=A^{1 \times 4} /\left(A^{1 \times 6} R\right), \\
M_{1}=\left(A^{1 \times 3} G_{11}\right) /\left(A^{1 \times 6} R\right), \\
M_{2}=\left(A^{1 \times 2} G_{21}\right) /\left(A^{1 \times 6} R\right), \\
M_{3}=\left(A G_{31}\right) /\left(A^{1 \times 6} R\right), \\
M_{4}=0,
\end{array}\right.
$$

where $G_{i 1}$ is the first matrix of the $i^{\text {th }}$ entry of $G$ (the second entry $G_{i 2}$ is the index $i$ of the submodule $M_{i}$ ). Equivalently, if $\pi: A^{1 \times 4} \longrightarrow M$ is the canonical projection, $\left\{f_{j}\right\}_{j=1, \ldots, 4}$ the standard basis of $A^{1 \times 4}$, and $\left\{y_{j}=\pi\left(f_{j}\right)\right\}_{j=1, \ldots, 4}$ a family of generators of $M$, then:

$$
\left\{\begin{array}{l}
M_{0}=A y_{1}+A y_{2}+A y_{3}+A y_{4}, \\
M_{1}=A\left(y_{1}-y_{3}\right)+A\left(y_{2}+y_{3}\right)+A\left(\left(-2 d_{2}+d_{3}+d_{1}\right) y_{3}-y_{4}\right), \\
M_{2}=A\left(y_{2}+y_{3}\right)+A\left(-y_{1}+y_{2}+2 y_{3}\right), \\
M_{3}=A\left(y_{1}-y_{2}-2 y_{3}\right), \\
M_{4}=0 .
\end{array}\right.
$$

If we add an option to the command GradeFiltrationByGenerators, then we also obtain the annihilators of the above family of generators of the $A$-modules $M_{i}$ 's (see Algorithm 2):

$$
\begin{aligned}
& >\text { GradeFiltrationByGenerators (R, A, opt); } \\
& \left.\left[\begin{array}{ccc}
4 d_{2}-d_{3} & 0 & 0 \\
4 d_{1}-d_{3} & 0 & 0 \\
0 & 4 d_{2}-d_{3} & 0 \\
0 & 4 d_{1}-d_{3} & 0 \\
0 & 0 & 4 d_{2}-d_{3} \\
0 & 0 & 4 d_{1}-d_{3}
\end{array}\right],\left[\begin{array}{cccc}
1 & 0 & -1 & 0 \\
0 & 1 & 1 & 0 \\
0 & 0 & -2 d_{2}+d_{3}+d_{1} & -1
\end{array}\right], 1\right] \\
& \left.\left.\left[\begin{array}{cc}
4 d_{2}-d_{3} & 0 \\
4 d_{1}-d_{3} & 0 \\
0 & d_{3} \\
0 & d_{2} \\
0 & d_{1}
\end{array}\right],\left[\begin{array}{cccc}
0 & 1 & 1 & 0 \\
-1 & 1 & 2 & 0
\end{array}\right], 2\right],\left[\left[\begin{array}{c}
d_{3} \\
d_{2} \\
d_{1}
\end{array}\right],\left[\begin{array}{llll}
1 & -1 & -2 & 0
\end{array}\right], 3\right]\right]
\end{aligned}
$$

The matrix in front of $G_{i 1}$ defines the PD operators which annihilate the generators of $M_{i}$ (which are defined by the residue class of the rows of $G_{i}$ in $M$ ). For instance, the first generator $z_{1}=y_{1}-y_{3}$ of $M_{1}$ satisfies $\left(4 d_{2}-d_{3}\right) z_{1}=0$ and $\left(4 d_{1}-d_{3}\right) z_{1}=0$ (similarly for the second $z_{2}=y_{2}+y_{3}$ and third generator $z_{3}=\left(-2 d_{2}+d_{3}+d_{1}\right) y_{3}-y_{4}$ of $\left.M_{1}\right)$. Similarly, $M_{2}$ is generated by $z_{2}$ and $z_{3}=-y_{1}+y_{2}+2 y_{3}$ which satisfies $d_{i} z_{3}=0$ for $i=1,2,3$. Finally, $z_{3}$ generates $M_{3}$ and satisfies $d_{i} z_{3}=0$ for $i=1,2,3$.

Another way to define the grade filtration $\left\{M_{i}\right\}_{i=0, \ldots, 3}$ of $M$ is by means of finitely presented $A$-modules $L_{i} \cong M_{i}$ and injective $\theta_{i} \in \operatorname{hom}_{A}\left(L_{i}, M\right)$ for $i=1,2,3$ (see Algorithm 3).

\section{$>\mathrm{H}:=$ GradeFiltrationByMorphisms $(\mathrm{R}, \mathrm{A})$;}




$$
\begin{aligned}
& H:=\left[\left[\begin{array}{ccc}
0 & 2 d_{1} & -1 \\
0 & 2 d_{2} & -1 \\
d_{3} & 0 & -2 \\
2 d_{2} & 0 & -1 \\
2 d_{1} & 0 & -1 \\
0 & d_{3} & -2 \\
0 & 0 & -d_{3}+4 d_{2} \\
0 & 0 & 4 d_{1}-d_{3}
\end{array}\right],\left[\begin{array}{cccc}
1 & 0 & -1 & 0 \\
0 & 1 & 1 & 0 \\
0 & 0 & -2 d_{2}+d_{3}+d_{1} & -1
\end{array}\right]\right] \\
& \left.\left[\begin{array}{ccc}
1 & 0 & 0 \\
0 & 0 & d_{1} \\
0 & 0 & d_{2} \\
0 & 0 & d_{3} \\
0 & 4 d_{1}-d_{3} & 0 \\
0 & -d_{3}+4 d_{2} & 0
\end{array}\right],\left[\begin{array}{cccc}
0 & -2 d_{1} & d_{3}-2 d_{2}-d_{1} & -1 \\
0 & 1 & 1 & 0 \\
-1 & 1 & 2 & 0
\end{array}\right],\left[\left[\begin{array}{c}
d_{1} \\
d_{2} \\
d_{3}
\end{array}\right],\left[\begin{array}{cccc}
1 & -1 & -2 & 0
\end{array}\right]\right]\right]
\end{aligned}
$$

We have $L_{1}=A^{1 \times 3} /\left(A^{1 \times 8} H_{11}\right), L_{2}=A^{1 \times 3} /\left(A^{1 \times 6} H_{21}\right)$, and $L_{3}=A /\left(A^{1 \times 3} H_{31}\right)$, where $H_{i 1}$ is the first matrix in the $i^{\text {th }}$ entry of $H$. Moreover, the injective $A$-homomorphism $\theta_{i}: L_{i} \longrightarrow M$ is defined by $\theta_{i}\left(\rho_{i}^{\prime}(\lambda)\right)=\pi\left(\lambda H_{i 2}\right)$, where $H_{i 2}$ is the second matrix in the $i^{\text {th }}$ entry of $H$ and $\rho_{i}^{\prime}$ is the canonical projection onto $L_{i}$. Let us check again that the $A$-homomorphisms $\theta_{i}$ 's are injective.

$>\operatorname{seq}(\operatorname{TestInj}(\mathrm{H}[\mathrm{i}][1], \mathrm{R}, \mathrm{H}[\mathrm{i}][2], \mathrm{A}), \mathrm{i}=1 \ldots 3)$;

true, true, true

Let us now compute a presentation of the pure $A$-modules $M_{i} / M_{i+1}$ for $i=0, \ldots, 3$ :

$>\mathrm{J}:=$ PureFactors_NR $(\mathrm{R}, \mathrm{A})$;

$\left.J:=\left[\begin{array}{cccc}1 & 0 & -1 & 0 \\ 0 & 1 & 1 & 0 \\ 0 & 0 & -2 d_{2}+d_{3}+d_{1} & -1\end{array}\right],\left[\begin{array}{ccc}0 & -2 d_{1} & 1 \\ 0 & 1 & 0 \\ -1 & 1 & 0\end{array}\right],\left[\begin{array}{ccc}0 & 0 & -1 \\ 1 & 0 & 0 \\ -1 & -4 d_{1}+d_{3} & 0 \\ 1 & -4 d_{1}+d_{3} & -d_{3} \\ 0 & d_{2}-d_{1} & 0 \\ 0 & d_{2}-d_{1} & -d_{2} \\ 0 & 0 & -d_{1}\end{array}\right],\left[\begin{array}{c}d_{2} \\ d_{3} \\ 0 \\ -d_{1}\end{array}\right]\right]$

If $J_{i}$ is the $i^{\text {th }}$ matrix of $J$, then $M / M_{1}=A^{1 \times 4} /\left(A^{1 \times 3} J_{1}\right), M_{1} / M_{2}=A^{1 \times 3} /\left(A^{1 \times 3} J_{2}\right)$, $M_{2} / M_{3}=A^{1 \times 3} /\left(A^{1 \times 7} J_{3}\right)$, and $M_{3}=A /\left(A^{1 \times 4} J_{4}\right)$.

Let us compute the codimension of the $A$-module $M_{i} / M_{i+1}$ for $i=0, \ldots, 2$ :

$>\operatorname{map}($ Codimension, $\mathrm{J}, \mathrm{A})$;

$$
[0, \infty, 2,3]
$$


In particular, we have $\operatorname{codim}_{A}\left(M_{1} / M_{2}\right)=\infty$, i.e., $M_{1}=M_{2}$. Let us now check that that the $A$-module $M_{i} / M_{i+1}$ is either 0 or $i$-pure for $i=0, \ldots, 3$ :

$>\operatorname{map}($ IsPure, F, A);

$$
[0, \infty, 2,3]
$$

The presentation matrix $J_{i}$ of $M_{i} / M_{i+1}$ does not generally form a Gröbner basis or is not simplified, which explains the suffix NR of the command PureFactors_NR, which stands for "NonReduced". To get such a presentation, we can use the command PureFactors_R, where R stands for "Reduced":

$$
\begin{aligned}
>K & :=\operatorname{PureFactors} \mathrm{R}(\mathrm{R}, \mathrm{A}) ; \\
K & \left.:=\left[\begin{array}{cccc}
1 & 0 & -1 & 0 \\
0 & 1 & 1 & 0 \\
0 & 0 & -2 d_{2}+d_{3}+d_{1} & -1
\end{array}\right],\left[\begin{array}{ccc}
0 & 1 & 0 \\
1 & 0 & 0 \\
0 & 0 & 1
\end{array}\right],\left[\begin{array}{ccc}
1 & 0 & 0 \\
0 & 0 & 1 \\
0 & 4 d_{1}-d_{3} & 0 \\
0 & -d_{3}+4 d_{2} & 0
\end{array}\right],\left[\begin{array}{c}
d_{1} \\
d_{2} \\
d_{3}
\end{array}\right]\right]
\end{aligned}
$$

Hence, $M / M_{1}=A^{1 \times 4} /\left(A^{1 \times 3} K_{1}\right), M_{1} / M_{2}=A^{1 \times 3} /\left(A^{1 \times 3} K_{2}\right), M_{2} / M_{3}=A^{1 \times 3} /\left(A^{1 \times 4} K_{3}\right)$, and $M_{3}=A /\left(A^{1 \times 3} K_{4}\right)$, where $K_{i}$ is the $i^{\text {th }}$ matrix of $K$.

We can simplify again the presentation of the $A$-module $M_{i} / M_{i+1}$ for $i=0, \ldots, 3$ by means of the elementary operations. This can be obtained by the command PureFactors.

$>\mathrm{F}:=\operatorname{PureFactors}(\mathrm{R}, \mathrm{A})$;

$$
F:=\left[[0],[1],\left[\begin{array}{c}
4 d_{1}-d_{3} \\
4 d_{2}-d_{3}
\end{array}\right],\left[\begin{array}{l}
d_{1} \\
d_{2} \\
d_{3}
\end{array}\right]\right]
$$

If $F_{i}$ is the $i^{\text {th }}$ matrix of $F$, then $M / M_{1} \cong A /\left(A F_{1}\right)=A, M_{1} / M_{2} \cong A /\left(A F_{2}\right)=A / A=0$, $M_{2} / M_{3} \cong A /\left(A^{1 \times 2} F_{3}\right)$, and $M_{3}=A /\left(A^{1 \times 3} F_{4}\right)$.

Let us check whether or not the $A$-module $M_{i} / M_{i+1}$ is 0 or $i$-pure for $i=0, \ldots, 3$.

$>\operatorname{map}($ IsPure $, \mathrm{K}, \mathrm{A})$;

$$
[0, \infty, 2,3]
$$

Let us compute a finite presentation of the $A$-module $M$ based on the presentation of the pure factors $M_{i} / M_{i+1}=\operatorname{coker}_{A}\left(. F_{i}\right)$ for $i=0, \ldots, 3$.

\section{$>\quad P:=$ PurePresentation_NR $(R, A)$ :}

We get that the $A$-module $M$ finitely presented by the matrix $P_{1}$ defined by

$>\mathrm{P}[1]$;

$$
\left[\begin{array}{cccc}
0 & -2 d_{1} & d_{3}-2 d_{2}-d_{1} & -1 \\
0 & d_{3}-2 d_{1} & 2 d_{2}-3 d_{1} & 1 \\
d_{3} & -6 d_{1} & -2 d_{2}-5 d_{1} & -1 \\
0 & d_{2}-d_{1} & d_{2}-d_{1} & 0 \\
d_{2} & -d_{1} & -d_{2}-d_{1} & 0 \\
d_{1} & -d_{1} & -2 d_{1} & 0
\end{array}\right]
$$


is isomorphic to the $A$-module $\bar{M}$ finitely presented by the matrix $P_{2}$ defined by:

P [2] ;
$\left.\qquad \begin{array}{ccccccccccc}1 & 0 & -1 & 0 & -1 & 0 & 0 & 0 & 0 & 0 & 0 \\ 0 & 1 & 1 & 0 & 0 & -1 & 0 & 0 & 0 & 0 & 0 \\ 0 & 0 & -2 d_{2}+d_{3}+d_{1} & -1 & 0 & 0 & -1 & 0 & 0 & 0 & 0 \\ 0 & 0 & 0 & 0 & 0 & -2 d_{1} & 1 & -1 & 0 & 0 & 0 \\ 0 & 0 & 0 & 0 & 0 & 1 & 0 & 0 & -1 & 0 & 0 \\ 0 & 0 & 0 & 0 & -1 & 1 & 0 & 0 & 0 & -1 & 0 \\ 0 & 0 & 0 & 0 & 0 & 0 & 0 & 0 & 0 & -1 & -1 \\ 0 & 0 & 0 & 0 & 0 & 0 & 0 & 1 & 0 & 0 & 0 \\ 0 & 0 & 0 & 0 & 0 & 0 & 0 & -1 & -4 d_{1}+d_{3} & 0 & 0 \\ 0 & 0 & 0 & 0 & 0 & 0 & 0 & 1 & -4 d_{1}+d_{3} & -d_{3} & 0 \\ 0 & 0 & 0 & 0 & 0 & 0 & 0 & 0 & d_{2}-d_{1} & 0 & 0 \\ 0 & 0 & 0 & 0 & 0 & 0 & 0 & 0 & d_{2}-d_{1} & -d_{2} & 0 \\ 0 & 0 & 0 & 0 & 0 & 0 & 0 & 0 & 0 & -d_{1} & 0 \\ 0 & 0 & 0 & 0 & 0 & 0 & 0 & 0 & 0 & 0 & d_{2} \\ 0 & 0 & 0 & 0 & 0 & 0 & 0 & 0 & 0 & 0 & d_{3} \\ 0 & 0 & 0 & 0 & 0 & 0 & 0 & 0 & 0 & 0 & 0 \\ 0 & 0 & 0 & 0 & 0 & 0 & 0 & 0 & 0 & 0 & -d_{1}\end{array}\right]$

In other words, $M=A^{1 \times 4} /\left(A^{1 \times 6} P_{1}\right) \cong \bar{M}=A^{1 \times 11} /\left(A^{1 \times 17} P_{2}\right)$ and $P_{2}$ is the block-triangular matrix defined in Theorem 12. The corresponding isomorphism is defined by the following matrix

$>\mathrm{P}[3]$;

$$
\left[\begin{array}{lllllllllll}
1 & 0 & 0 & 0 & 0 & 0 & 0 & 0 & 0 & 0 & 0 \\
0 & 1 & 0 & 0 & 0 & 0 & 0 & 0 & 0 & 0 & 0 \\
0 & 0 & 1 & 0 & 0 & 0 & 0 & 0 & 0 & 0 & 0 \\
0 & 0 & 0 & 1 & 0 & 0 & 0 & 0 & 0 & 0 & 0
\end{array}\right]
$$

i.e., $\varphi: M \longrightarrow \bar{M}$ is defined by $\varphi(\pi(\lambda))=\bar{\pi}\left(\lambda P_{3}\right)$, where $\bar{\pi}: A^{1 \times 11} \longrightarrow \bar{M}$ is the canonical projection onto $\bar{M}$. Let us check again that $\varphi$ is an isomorphism.

$$
>\text { TestIso(R,P[2],P[3],A); }
$$

true

Moreover, $\varphi^{-1}$ is defined by $\varphi^{-1}(\bar{\pi}(\mu))=\pi\left(\mu P_{4}\right)$, where $P_{4}$ is defined by:

$>\mathrm{P}[4]$; 


$$
\left[\begin{array}{cccc}
1 & 0 & 0 & 0 \\
0 & 1 & 0 & 0 \\
0 & 0 & 1 & 0 \\
0 & 0 & 0 & 1 \\
1 & 0 & -1 & 0 \\
0 & 1 & 1 & 0 \\
0 & 0 & -2 d_{2}+d_{3}+d_{1} & -1 \\
0 & -2 d_{1} & d_{3}-2 d_{2}-d_{1} & -1 \\
0 & 1 & 1 & 0 \\
-1 & 1 & 2 & 0 \\
1 & -1 & -2 & 0
\end{array}\right]
$$

Let us check again that the $A$-homomorphism from $\bar{M}$ to $M$ defined by $P_{4}$ is an isomorphism.

$>\operatorname{TestIso}(\mathrm{P}[2], \mathrm{R}, \mathrm{P}[4], \mathrm{A})$;

$$
\text { true }
$$

Let us now compute another presentation matrix $Q$ of the $A$-module $M$ whose diagonal blocks are the presentation matrices $K_{i}$ 's of the pure $A$-modules $M_{i} / M_{i+1}$ 's.

$>Q:=$ PurePresentation_R $(\mathrm{R}, \mathrm{A})$ :

We get that the $A$-module $M$ finitely presented by the matrix $Q_{1}$ defined by

$>\mathrm{Q}[1]$;

$$
\left[\begin{array}{cccc}
0 & -2 d_{1} & d_{3}-2 d_{2}-d_{1} & -1 \\
0 & d_{3}-2 d_{1} & 2 d_{2}-3 d_{1} & 1 \\
d_{3} & -6 d_{1} & -2 d_{2}-5 d_{1} & -1 \\
0 & d_{2}-d_{1} & d_{2}-d_{1} & 0 \\
d_{2} & -d_{1} & -d_{2}-d_{1} & 0 \\
d_{1} & -d_{1} & -2 d_{1} & 0
\end{array}\right]
$$

is isomorphic to the $A$-module $\overline{\bar{M}}$ finitely presented by the matrix $Q_{2}$ defined by

$>\mathrm{Q}[2]$; 


$$
\left[\begin{array}{ccccccccccc}
1 & 0 & -1 & 0 & -1 & 0 & 0 & 0 & 0 & 0 & 0 \\
0 & 1 & 1 & 0 & 0 & -1 & 0 & 0 & 0 & 0 & 0 \\
0 & 0 & -2 d_{2}+d_{3}+d_{1} & -1 & 0 & 0 & -1 & 0 & 0 & 0 & 0 \\
0 & 0 & 0 & 0 & 0 & 1 & 0 & 0 & -1 & 0 & 0 \\
0 & 0 & 0 & 0 & 1 & 0 & 0 & 0 & -1 & 1 & 0 \\
0 & 0 & 0 & 0 & 0 & 0 & 1 & -1 & -2 d_{1} & 0 & 0 \\
0 & 0 & 0 & 0 & 0 & 0 & 0 & 1 & 0 & 0 & 0 \\
0 & 0 & 0 & 0 & 0 & 0 & 0 & 0 & 0 & 1 & 1 \\
0 & 0 & 0 & 0 & 0 & 0 & 0 & 0 & 4 d_{1}-d_{3} & 0 & 0 \\
0 & 0 & 0 & 0 & 0 & 0 & 0 & 0 & -d_{3}+4 d_{2} & 0 & 0 \\
0 & 0 & 0 & 0 & 0 & 0 & 0 & 0 & 0 & 0 & d_{1} \\
0 & 0 & 0 & 0 & 0 & 0 & 0 & 0 & 0 & 0 & d_{2} \\
0 & 0 & 0 & 0 & 0 & 0 & 0 & 0 & 0 & 0 & d_{3}
\end{array}\right]
$$

i.e., $M=A^{1 \times 4} /\left(A^{1 \times 6} Q_{1}\right) \cong \overline{\bar{M}}=A^{1 \times 11} /\left(A^{1 \times 13} Q_{2}\right)$. The isomorphism $\psi: M \longrightarrow \overline{\bar{M}}$ is defined by the following matrix

$>\mathrm{Q}[3]$;

$$
\left[\begin{array}{ccccccccccc}
0 & 0 & 1 & 0 & 0 & 0 & 0 & 0 & 1 & 0 & 1 \\
0 & 0 & -1 & 0 & 0 & 0 & 0 & 0 & 1 & 0 & 0 \\
0 & 0 & 1 & 0 & 0 & 0 & 0 & 0 & 0 & 0 & 0 \\
0 & 0 & 0 & 1 & 0 & 0 & 0 & 0 & 0 & 0 & 0
\end{array}\right]
$$

i.e., $\psi(\pi(\lambda))=\overline{\bar{\pi}}\left(\lambda Q_{3}\right)$. Let us check again that $\psi$ is an isomorphism.

$>\operatorname{TestIso}(\mathrm{R}, \mathrm{Q}[2], \mathrm{Q}[3], \mathrm{A})$;

true

Moreover, $\psi^{-1}: \overline{\bar{M}} \longrightarrow M$ is defined by $\psi^{-1}(\overline{\bar{\pi}}(\mu))=\pi\left(\mu Q_{4}\right)$, where $Q_{4}$ is defined by:

$>\mathrm{Q}[4]$; 


$$
\left[\begin{array}{cccc}
1 & 0 & 0 & 0 \\
0 & 1 & 0 & 0 \\
0 & 0 & 1 & 0 \\
0 & 0 & 0 & 1 \\
1 & 0 & -1 & 0 \\
0 & 1 & 1 & 0 \\
0 & 0 & -2 d_{2}+d_{3}+d_{1} & -1 \\
0 & -2 d_{1} & d_{3}-2 d_{2}-d_{1} & -1 \\
0 & 1 & 1 & 0 \\
-1 & 1 & 2 & 0 \\
1 & -1 & -2 & 0
\end{array}\right]
$$

Let us check again that $\psi^{-1}$ is an isomorphism.

$>$ TestIso(Q[2], R, Q[4], A);

true

We can simplify again the presentation matrix $Q_{2}$ by means of elementary operations. This can be achieved using the command PurePresentation.

$>\mathrm{S}:=$ PurePresentation $(\mathrm{R}, \mathrm{A})$;

$$
\begin{gathered}
S:=\left[\begin{array}{cccc}
0 & -2 d_{1} & d_{3}-2 d_{2}-d_{1} & -1 \\
0 & d_{3}-2 d_{1} & 2 d_{2}-3 d_{1} & 1 \\
d_{3} & -6 d_{1} & -2 d_{2}-5 d_{1} & -1 \\
0 & d_{2}-d_{1} & d_{2}-d_{1} & 0 \\
d_{2} & -d_{1} & -d_{2}-d_{1} & 0 \\
d_{1} & -d_{1} & -2 d_{1} & 0
\end{array}\right],\left[\begin{array}{ccc}
-4 d_{1}+d_{3} & 0 & 0 \\
-4 d_{2}+d_{3} & 0 & 0 \\
0 & 0 & d_{1} \\
0 & 0 & d_{2} \\
0 & 0 & d_{3}
\end{array}\right], \\
\left.\left[\begin{array}{ccc}
-1 & -1 & 1 \\
-1 & 1 & 0 \\
0 & -1 & 0 \\
2 d_{1} & 2 d_{2}-d_{1}-d_{3} & 0
\end{array}\right],\left[\begin{array}{cccc}
0 & -1 & -1 & 0 \\
0 & 0 & -1 & 0 \\
1 & -1 & -2 & 0
\end{array}\right]\right]
\end{gathered}
$$

We obtain $M \cong L=A^{1 \times 3} /\left(A^{1 \times 5} S_{2}\right)$, where $S_{2}$ is the second matrix of $S$. The isomorphism $\varphi: M \longrightarrow L$ is defined by $\varphi(\pi(\lambda))=\vartheta\left(\lambda S_{3}\right)$, where $S_{3}$ is the third matrix of $S, \lambda \in A^{1 \times 4}$, and $\vartheta: A^{1 \times 3} \longrightarrow L$ is the canonical projection onto $L$.

Let us check again that $\varphi$ is an isomorphism.

$>$ TestIso $(\mathrm{R}, \mathrm{S}[2], \mathrm{S}[3], \mathrm{A})$;

\section{true}

Moreover, $\varphi^{-1}: L \longrightarrow M$ is defined $\varphi^{-1}(\vartheta(\mu))=\pi\left(\mu S_{4}\right)$ for all $\mu \in A^{1 \times 3}$, where $S_{4}$ is the fourth matrix of $S$. 
$>$ TestIso(S[2], R, S[4], A);

true

From the presentation matrix $S$, we get $M \cong M_{3} \oplus M_{1} / M_{3} \oplus A$.

A presentation of the torsion submodule $t(M)=M_{1}$ of $M$ based on the terms $\left\{M_{i}\right\}_{i=1,2,3}$ of the grade filtration of $M_{1}$ can be computed using the command PurePresentationOfTorsionSubmodule.

$>\mathrm{T}:=$ PurePresentationOfTorsionSubmodule $(\mathrm{R}, \mathrm{A})$;

$$
\left.T:=\left[\begin{array}{cc}
4 d_{1}-d_{3} & 0 \\
4 d_{2}-d_{3} & 0 \\
0 & d_{1} \\
0 & d_{2} \\
0 & d_{3}
\end{array}\right],\left[\begin{array}{cccc}
0 & -2 d_{1} & d_{3}-2 d_{2}-d_{1} & -1 \\
0 & d_{3}-2 d_{1} & 2 d_{2}-3 d_{1} & 1 \\
d_{3} & -6 d_{1} & -2 d_{2}-5 d_{1} & -1 \\
0 & d_{2}-d_{1} & d_{2}-d_{1} & 0 \\
d_{2} & -d_{1} & -d_{2}-d_{1} & 0 \\
d_{1} & -d_{1} & -2 d_{1} & 0
\end{array}\right],\left[\begin{array}{cccc}
0 & 1 & 1 & 0 \\
1 & -1 & -2 & 0
\end{array}\right]\right]
$$

We can check that the $A$-homomorphism $\iota: t(M)=A^{1 \times 2} /\left(A^{1 \times 5} T_{1}\right) \longrightarrow M=A^{1 \times 4} /\left(A^{1 \times 6} T_{2}\right)$ defined by $\iota(\sigma(\nu))=\pi\left(\nu T_{3}\right)$ is injective.

$>\operatorname{TestInj}(\mathrm{T}[1], \mathrm{T}[2], \mathrm{T}[3], \mathrm{A})$;

true

Let us check that the $A$-module finitely presented by $T_{1}$ is torsion.

$>$ Codimension $(\mathrm{T}[1], \mathrm{A})$;

2

Let us compute a solution of the linear system $\operatorname{ker}_{\mathcal{F}}\left(T_{1}.\right) \cong \operatorname{hom}_{D}(t(M), \mathcal{F})$.

$>\mathrm{z}$ :=IntegrationOfTorsionDSubmodule $(\mathrm{R}, \mathrm{A})$;

$$
z:=\left[\begin{array}{c}
-F 1\left(1 / 4 x_{2}+1 / 4 x_{1}+x_{3}\right) \\
-C 1
\end{array}\right]
$$

Let us check that $z$ is a solution of $\operatorname{ker}_{\mathcal{F}}\left(T_{1}.\right)$.

$>\operatorname{ApplyMatrix}(\mathrm{T}[1], \mathrm{z}, \mathrm{A})$;

$$
\left[\begin{array}{l}
0 \\
0 \\
0 \\
0 \\
0
\end{array}\right]
$$

Finally, let us try to integrate the linear system $\operatorname{ker}_{\mathcal{F}}(R.) \cong \operatorname{hom}_{D}(M, \mathcal{F})$. We obtain $>\mathrm{y}:=$ IntegrationOfDModule $(\mathrm{R}, \mathrm{A}, \mathrm{xi})$; 


$$
y:=\left[\begin{array}{c}
-y_{3}\left(x_{1}, x_{2}, x_{3}\right)+{ }_{-} F 1\left(1 / 4 x_{2}+1 / 4 x_{1}+x_{3}\right)+{ }_{-} C 1-\xi_{1}\left(x_{1}, x_{2}, x_{3}\right) \\
-{ }_{-} y_{3}\left(x_{1}, x_{2}, x_{3}\right)+{ }_{-} F 1\left(1 / 4 x_{2}+1 / 4 x_{1}+x_{3}\right)+\xi_{1}\left(x_{1}, x_{2}, x_{3}\right) \\
-y_{3}\left(x_{1}, x_{2}, x_{3}\right)-\xi_{1}\left(x_{1}, x_{2}, x_{3}\right) \\
-1 / 2 \mathrm{D}\left({ }_{-} F 1\right)\left(1 / 4 x_{2}+1 / 4 x_{1}+x_{3}\right)+\frac{\partial}{\partial x_{1}}-y_{3}\left(x_{1}, x_{2}, x_{3}\right)-2 \frac{\partial}{\partial x_{2}} y_{3}\left(x_{1}, x_{2}, x_{3}\right) \\
+\frac{\partial}{\partial x_{3}} y_{3}\left(x_{1}, x_{2}, x_{3}\right)-\frac{\partial}{\partial x_{1}} \xi_{1}\left(x_{1}, x_{2}, x_{3}\right)+2 \frac{\partial}{\partial x_{2}} \xi_{1}\left(x_{1}, x_{2}, x_{3}\right)-\frac{\partial}{\partial x_{3}} \xi_{1}\left(x_{1}, x_{2}, x_{3}\right)
\end{array}\right]
$$

where $\xi$ and $y_{3}$ are two arbitrary functions of $x_{1}, x_{2}, x_{3}$ (their difference can be replaced in $y$ by a single function of $\left.x_{1}, x_{2}, x_{3}\right), F_{1}$ an arbitrary function of 1 variable, and $C_{1}$ an arbitrary constant. Let us finally check that $y$ is a solution of $\operatorname{ker}_{\mathcal{F}}(R$.).

$>$ ApplyMatrix $(\mathrm{R}, \mathrm{y}, \mathrm{A})$;

$$
\left[\begin{array}{l}
0 \\
0 \\
0 \\
0 \\
0 \\
0
\end{array}\right]
$$

Example 12. Let $A$ be the ring of PD operators in $d_{1}=\frac{\partial}{\partial x_{1}}, d_{2}=\frac{\partial}{\partial x_{2}}, d_{3}=\frac{\partial}{\partial x_{3}}$, and $d_{4}=\frac{\partial}{\partial x_{4}}$ with coefficients in $\mathbb{Q}\left[x_{1}, x_{2}, x_{3}, x_{4}\right]$

$>A:=$ DefineOreAlgebra (diff $=[d[1], x[1]], \operatorname{diff}=[d[2], x[2]], \operatorname{diff}=[d[3], x[3]]$,

$>\operatorname{diff}=[d[4], x[4]]$, polynom $=[x[1], x[2], x[3], x[4]]):$

and the linearized Einstein equations in the vacuum defined by the following matrix $R \in A^{10 \times 10}$ :

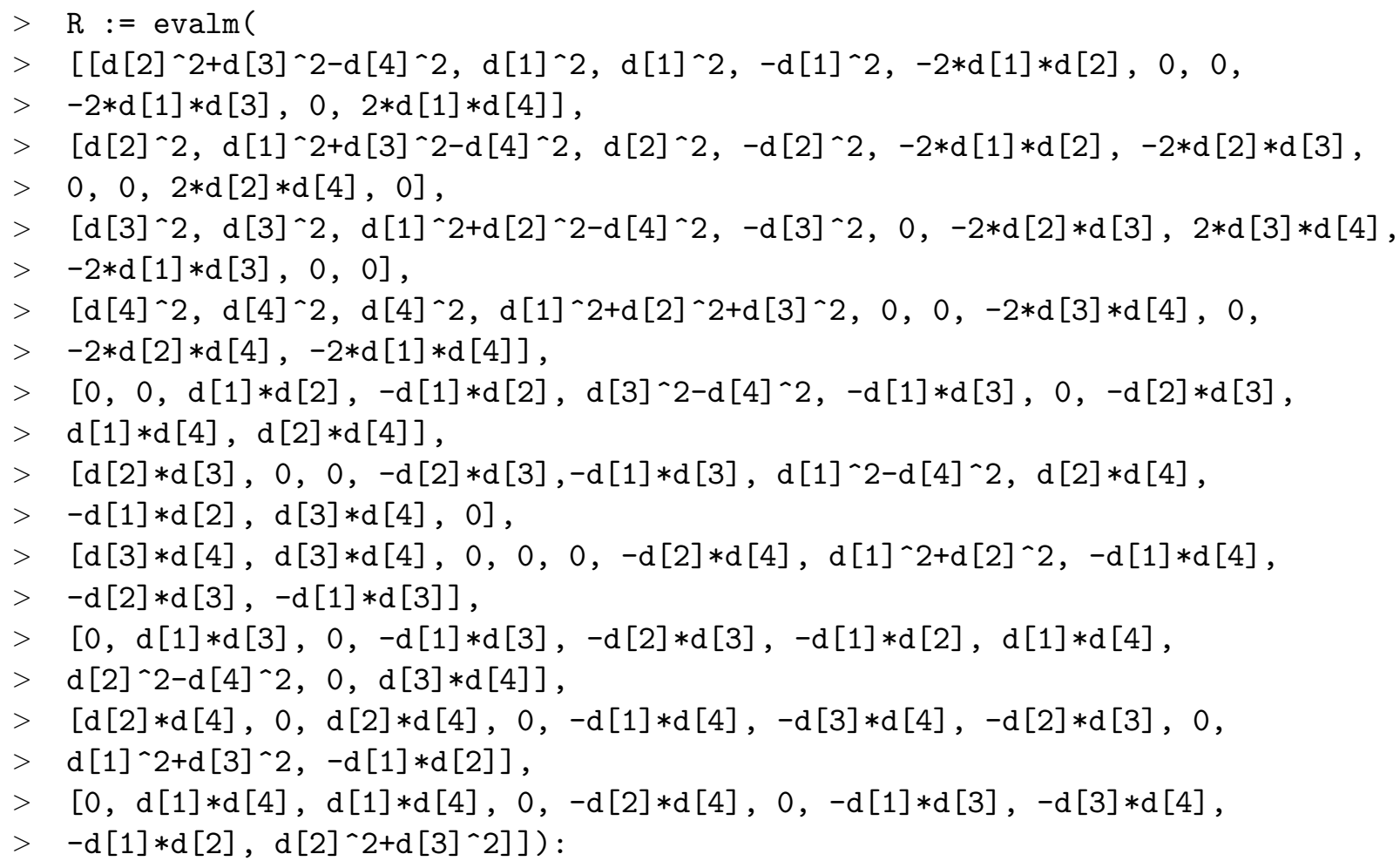


Let $M=A^{1 \times 10} /\left(A^{1 \times 10} R\right)$ be the $A$-module finitely presented by $R$. Let us first compute the codimension of $M$.

$>$ Codimension $(\mathrm{R}, \mathrm{A})$;

0

We get $\operatorname{codim}_{A}(M)=0$, i.e., $\operatorname{dim}_{A}(M)=4$. Let us check that $j_{A}(M)=\operatorname{codim}_{A}(M)$.

\section{$>$ GradeNumber $(\mathrm{R}, \mathrm{A})$;}

0

Let us now compute the grade filtration $\left\{M_{i}\right\}_{i=0, \ldots, 4}$ of $M$.

$>\mathrm{G}:=$ GradeFiltrationByGenerators $(\mathrm{R}, \mathrm{A})$ :

We get a 2-step filtration of $M$ since $G$ contains only 2 elements:

$>\operatorname{nops}(G)$;

2

The $A$-module $M_{1}=t(M)$ is defined by the residue classes of the rows of the first matrix of the first entry $G_{1}$ of $G$ defined by:

$$
\begin{aligned}
& >\mathrm{G}[1] \text {; }
\end{aligned}
$$

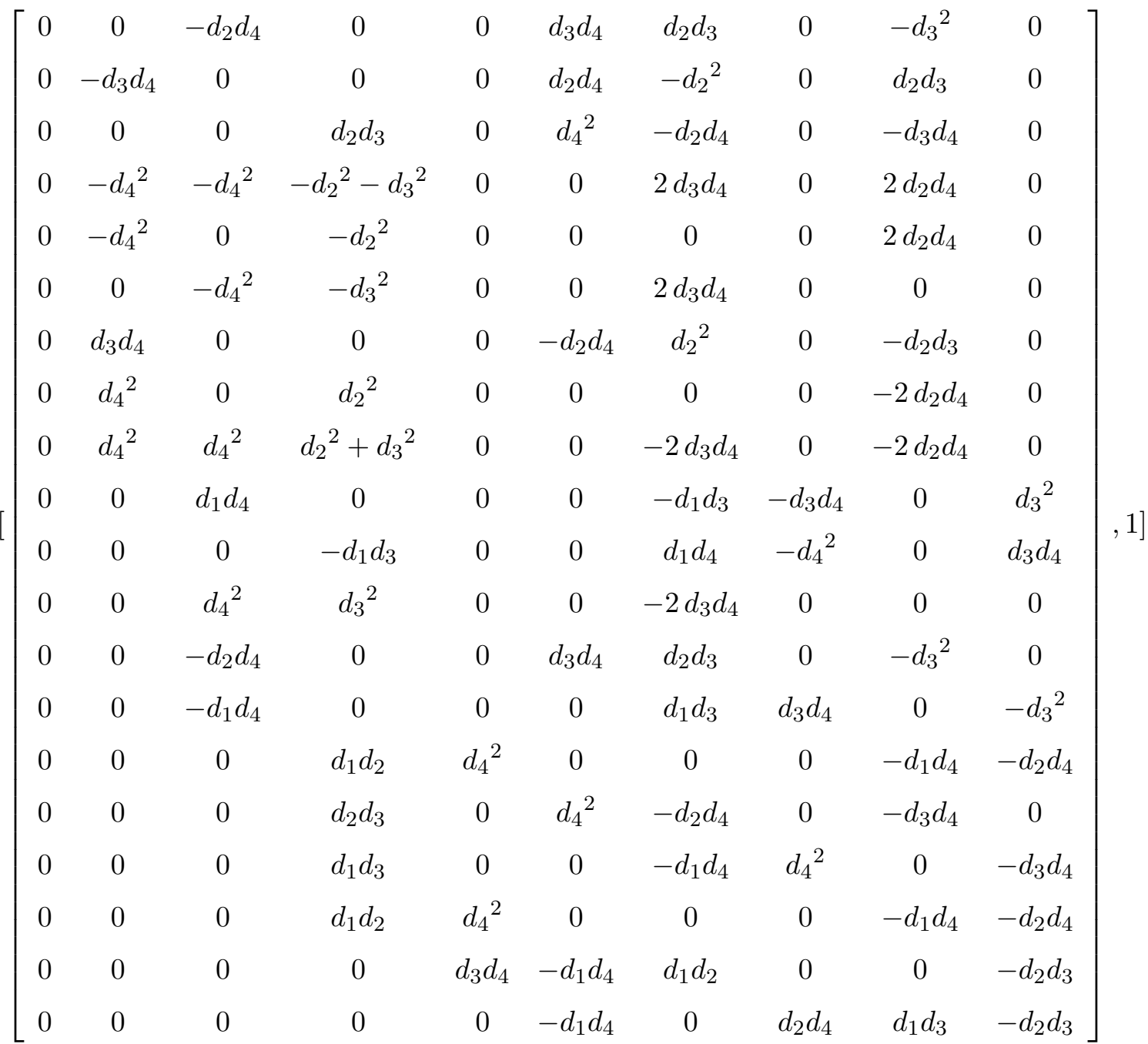

$\mathrm{RR} \mathrm{n}^{\circ} 7769$ 
In other words, we have $t(M)=M_{1}=\left(A^{1 \times 20} G_{11}\right) /\left(A^{1 \times 10} R\right)$, where $G_{11}$ is the first entry of $G_{1}$. Since we have

$>\mathrm{G}[2]$;

[[], 2]

we get $M_{2}=0$, which shows that the grade filtration of $M$ is $0=M_{2} \subseteq M_{1} \subseteq M$.

Let us now compute a presentation of the pure $A$-modules $M / M_{1}$ and $M_{1}$.

\section{$>\mathrm{F}:=$ PureFactors $(\mathrm{R}, \mathrm{A})$ :}

Let us check whether or not the $A$-module $M_{i} / M_{i+1}$ is 0 or $i$-pure for $i=0,1,2$.

$>\operatorname{map}($ IsPure, $\mathrm{F}, \mathrm{A})$;

$$
[0,1, \infty]
$$

We obtain that 0 -pure $A$-module $M / M_{1}=M / t(M)$ is finitely presented by the following matrix:

$$
\begin{aligned}
& >\mathrm{F}[1] \text {; } \\
& {\left[\begin{array}{cccccccccc}
d_{2}{ }^{2} & d_{1}{ }^{2} & 0 & 0 & -2 d_{1} d_{2} & 0 & 0 & 0 & 0 & 0 \\
d_{3}{ }^{2} & 0 & d_{1}{ }^{2} & 0 & 0 & 0 & 0 & -2 d_{1} d_{3} & 0 & 0 \\
d_{4}{ }^{2} & 0 & 0 & d_{1}{ }^{2} & 0 & 0 & 0 & 0 & 0 & -2 d_{1} d_{4} \\
0 & d_{3}{ }^{2} & d_{2}{ }^{2} & 0 & 0 & -2 d_{2} d_{3} & 0 & 0 & 0 & 0 \\
0 & d_{4}{ }^{2} & 0 & d_{2}{ }^{2} & 0 & 0 & 0 & 0 & -2 d_{2} d_{4} & 0 \\
0 & 0 & d_{4}{ }^{2} & d_{3}{ }^{2} & 0 & 0 & -2 d_{3} d_{4} & 0 & 0 & 0 \\
d_{2} d_{3} & 0 & 0 & 0 & -d_{1} d_{3} & d_{1}{ }^{2} & 0 & -d_{1} d_{2} & 0 & 0 \\
d_{2} d_{4} & 0 & 0 & 0 & -d_{1} d_{4} & 0 & 0 & 0 & d_{1}{ }^{2} & -d_{1} d_{2} \\
d_{3} d_{4} & 0 & 0 & 0 & 0 & 0 & d_{1}{ }^{2} & -d_{1} d_{4} & 0 & -d_{1} d_{3} \\
0 & d_{1} d_{3} & 0 & 0 & -d_{2} d_{3} & -d_{1} d_{2} & 0 & d_{2}{ }^{2} & 0 & 0 \\
0 & d_{1} d_{4} & 0 & 0 & -d_{2} d_{4} & 0 & 0 & 0 & -d_{1} d_{2} & d_{2}{ }^{2} \\
0 & d_{3} d_{4} & 0 & 0 & 0 & -d_{2} d_{4} & d_{2}{ }^{2} & 0 & -d_{2} d_{3} & 0 \\
0 & 0 & d_{1} d_{2} & 0 & d_{3}{ }^{2} & -d_{1} d_{3} & 0 & -d_{2} d_{3} & 0 & 0 \\
0 & 0 & d_{1} d_{4} & 0 & 0 & 0 & -d_{1} d_{3} & -d_{3} d_{4} & 0 & d_{3}{ }^{2} \\
0 & 0 & d_{2} d_{4} & 0 & 0 & -d_{3} d_{4} & -d_{2} d_{3} & 0 & d_{3}{ }^{2} & 0 \\
0 & 0 & 0 & d_{1} d_{2} & d_{4}{ }^{2} & 0 & 0 & 0 & -d_{1} d_{4} & -d_{2} d_{4} \\
0 & 0 & 0 & d_{1} d_{3} & 0 & 0 & -d_{1} d_{4} & d_{4}{ }^{2} & 0 & -d_{3} d_{4} \\
0 & 0 & 0 & d_{2} d_{3} & 0 & d_{4}{ }^{2} & -d_{2} d_{4} & 0 & -d_{3} d_{4} & 0 \\
0 & 0 & 0 & 0 & 0 & d_{1} d_{4} & 0 & -d_{2} d_{4} & -d_{1} d_{3} & d_{2} d_{3} \\
0 & 0 & 0 & 0 & d_{3} d_{4} & 0 & d_{1} d_{2} & -d_{2} d_{4} & -d_{1} d_{3} & 0
\end{array}\right]}
\end{aligned}
$$

Moreover, the 1-pure $A$-module $M_{1}=t(M)$ is finitely presented by the matrix $F_{2}$. Since $F_{2}$ is a large matrix, let us print it in pieces. 


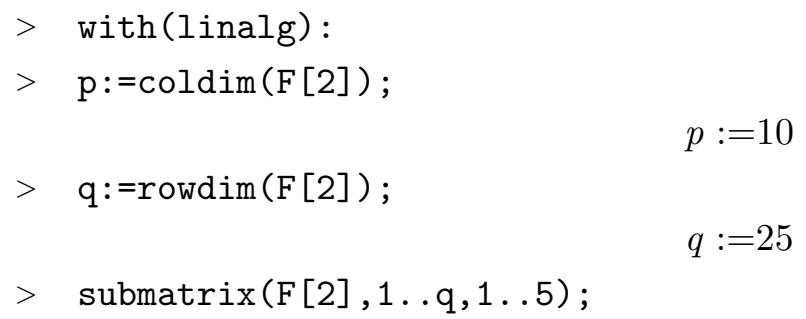




\begin{tabular}{|c|c|c|c|c|}
\hline 0 & $-d_{3}$ & 0 & 0 & 0 \\
\hline$-d_{3}$ & 0 & 0 & 0 & 0 \\
\hline$d_{1}$ & 0 & $-d_{3}$ & $d_{4}$ & 0 \\
\hline 0 & 0 & 0 & 0 & 0 \\
\hline 0 & 0 & $d_{4}$ & $-d_{3}$ & 0 \\
\hline 0 & $d_{2}$ & $-d_{3}$ & $d_{4}$ & $-d_{4}$ \\
\hline 0 & $d_{3}$ & $d_{2}$ & 0 & 0 \\
\hline 0 & 0 & $d_{4}$ & $-d_{3}$ & $d_{3}$ \\
\hline$d_{4}$ & 0 & 0 & $d_{1}$ & 0 \\
\hline$-d_{4}$ & 0 & 0 & 0 & $-d_{1}$ \\
\hline 0 & $-d_{4}$ & 0 & 0 & $d_{2}$ \\
\hline 0 & $-d_{4}$ & 0 & $-d_{2}$ & $d_{2}$ \\
\hline 0 & 0 & 0 & 0 & 0 \\
\hline 0 & $d_{1}$ & 0 & 0 & 0 \\
\hline 0 & 0 & $-d_{1}$ & 0 & 0 \\
\hline$d_{2}$ & $d_{1}$ & 0 & 0 & 0 \\
\hline 0 & $-d_{1} d_{3}$ & 0 & 0 & 0 \\
\hline 0 & 0 & $2 d_{3} d_{4}$ & $-d_{4}^{2}-d_{3}^{2}$ & $-d_{2}^{2}+d_{4}^{2}$ \\
\hline 0 & 0 & 0 & $d_{1}^{2}+d_{2}^{2}+d_{3}^{2}-d_{4}^{2}$ & 0 \\
\hline$d_{3} d_{4}$ & 0 & $-d_{1} d_{4}$ & $d_{1} d_{3}$ & 0 \\
\hline 0 & $d_{3} d_{4}$ & $-d_{2} d_{4}$ & $d_{2} d_{3}$ & $-d_{2} d_{3}$ \\
\hline 0 & 0 & 0 & 0 & $d_{1}^{2}+d_{2}^{2}+d_{3}^{2}-d_{4}^{2}$ \\
\hline 0 & 0 & $d_{1}^{2}+d_{2}^{2}+d_{3}^{2}-d_{4}^{2}$ & 0 & 0 \\
\hline$d_{2}^{2}+d_{3}^{2}-d_{4}^{2}$ & 0 & $d_{1} d_{3}$ & $-d_{1} d_{4}$ & 0 \\
\hline 0 & $d_{1}^{2}+d_{3}^{2}-d_{4}^{2}$ & $d_{2} d_{3}$ & $-d_{2} d_{4}$ & $d_{2} d_{4}$ \\
\hline
\end{tabular}

\subsection{Equidimensional decomposition of affine algebraic varieties}

Example 13. Let us consider the commutative polynomial ring $A=\mathbb{Q}[x, y, z]$

$>A:=$ DefineOreAlgebra $(\operatorname{diff}=[x, s 1], \operatorname{diff}=[y, s 2], \operatorname{dif} f=[z, s 3]$, polynom $=[\mathrm{s} 1, \mathrm{~s} 2, \mathrm{~s} 3]):$ and the matrix $R \in A^{1 \times 3}$ defined by:

$$
\begin{aligned}
& >\quad \mathrm{R}:=e v a \operatorname{lm}\left(\left[\left[\mathrm{x}^{\wedge} 3+\mathrm{x}^{\wedge} 2 * \mathrm{y}+\mathrm{x}^{\wedge} 2 * \mathrm{z}-\mathrm{x}^{\wedge} 2-\mathrm{x} * \mathrm{z}-\mathrm{y} * \mathrm{z}-\mathrm{z}^{\wedge} 2+\mathrm{z}\right],\left[\mathrm{x}^{\wedge} 2 * \mathrm{y} * \mathrm{z}+\mathrm{x}^{\wedge} 2 * \mathrm{y}-\mathrm{y} * \mathrm{z}^{\wedge} 2-\mathrm{y} * \mathrm{z}\right]\right.\right. \text {, } \\
& \left.\left.>\left[\mathrm{x}^{\wedge} 2 * \mathrm{y}^{\wedge} 2-\mathrm{x}^{\wedge} 2 * \mathrm{y}-\mathrm{y}^{\wedge} 2 * \mathrm{z}+\mathrm{y} * \mathrm{z}\right]\right]\right) \text {; } \\
& R:=\left[\begin{array}{c}
x^{3}+x^{2} y+x^{2} z-x^{2}-x z-y z-z^{2}+z \\
x^{2} y z+x^{2} y-y z^{2}-y z \\
x^{2} y^{2}-x^{2} y-y^{2} z+y z
\end{array}\right]
\end{aligned}
$$

Let us consider the $A$-module $M=A / I$, where $I=A^{1 \times 3} R$ is the ideal of $A$ generated by the three entries of $R$. The $A$-module $M$ was first considered in Exercise 4.4.5 of G.-M. Greuel, G. Pfister, "A Singular Introduction to Commutative Algebra", Springer, 2002, p. 261. 
Let us first try to solve the polynomial system defined by $I$ using the Maple command solve:

$$
\begin{aligned}
& >\operatorname{solve}(\operatorname{convert}(\mathrm{R}, \mathrm{set})) ; \\
& \left\{\begin{array}{c}
\left\{x=x, y=y, z=x^{2}\right\},\left\{x=\operatorname{RootOf}\left(Z^{2}+1\right), y=y, z=-1\right\}, \\
\{x=x, y=0, z=-x+1\},\{x=1, y=1, z=-1\}
\end{array}\right.
\end{aligned}
$$

The Maple output shows that the complex algebraic variety $V(I)$ defined by the ideal $I$ is formed by a point, 3 curves and a hypersurface. In particular, $V(I)$ is not equidimensional. Hence, let us check again that $M$ is not a pure $A$-module.

$>\operatorname{IsPure}(\mathrm{R}, \mathrm{A})$;

$$
\text { false }
$$

Let us now compute the grade filtration of $M$.

$>\mathrm{G}:=$ GradeFiltrationByGenerators $(\mathrm{R}, \mathrm{A})$;

$$
G:=\left[[[1], 1],\left[\left[x^{2}-z\right], 2\right],\left[\left[x^{2} y-y z\right], 3\right]\right]
$$

If $\pi: A \longrightarrow M$ is the canonical projection onto $M$ and $u=\pi(1)$ the generator of $M$, then we have $M_{1}=A u=M, M_{2}=A\left(x^{2}-z\right) u, M_{3}=A\left(y\left(x^{2}-z\right) u\right)$, and $M_{4}=0$.

If an option is added to the command GradeFiltrationByGenerators, then the annihilator of the generators of the $A$-modules $M_{i}$ 's are also computed and returned in the first matrix of each entry of the output.

$$
\begin{gathered}
>\mathrm{H}:=\text { GradeFiltrationByGenerators }(\mathrm{R}, \mathrm{A}, \mathrm{opt}) \\
\left.H:=\left[\left[\begin{array}{c}
x^{3}+x^{2} y+x^{2} z-x^{2}-x z-y z-z^{2}+z \\
x^{2} y z+x^{2} y-y z^{2}-y z \\
x^{2} y^{2}-x^{2} y-y^{2} z+y z
\end{array}\right],[1], 1\right],\left[\begin{array}{c}
x+y+z-1 \\
y z+y \\
y^{2}-y
\end{array}\right],\left[x^{2}-z\right], 2\right], \\
\left.\left.\left[\begin{array}{c}
z+1 \\
y-1 \\
x-1
\end{array}\right],\left[x^{2} y-y z\right], 3\right]\right]
\end{gathered}
$$

Hence, we get $M_{1} \cong A /\left(A^{1 \times 3} H_{11}\right)=M, M_{2} \cong A /\left(A^{1 \times 3} H_{21}\right)$, and $M_{3} \cong A /\left(A^{1 \times 3} H_{31}\right)$, where $H_{i 1}$ is the first matrix in the $i^{\text {th }}$ entry of $H$.

Another way to define the grade filtration $\left\{M_{i}\right\}_{i=0, \ldots, 3}$ of $M$ is by means of finitely presented $A$-modules $L_{i} \cong M_{i}$ and injective $\theta_{i} \in \operatorname{hom}_{A}\left(L_{i}, M\right)$ for $i=1,2,3$ (see Algorithm 3 ).

$$
\begin{aligned}
& >\mathrm{J}:=\text { GradeFiltrationByMorphisms }(\mathrm{R}, \mathrm{A}) \text {; } \\
& J:=\left[\left[\left[\begin{array}{c}
x^{2} y^{2}-x^{2} y-y^{2} z+y z \\
x^{2} y z+x^{2} y-y z^{2}-y z \\
x^{3}+x^{2} y+x^{2} z-x^{2}-x z-y z-z^{2}+z
\end{array}\right],[1]\right],\left[\left[\begin{array}{c}
y^{2}-y \\
y z+y \\
x+y+z-1
\end{array}\right],\left[x^{2}-z\right]\right],\right. \\
& \left.\left.\left[\begin{array}{c}
y-1 \\
z+1 \\
x-1
\end{array}\right],\left[y\left(x^{2}-z\right)\right]\right]\right]
\end{aligned}
$$


We obtain $L_{1}=A /\left(A^{1 \times 3} J_{11}\right), L_{2}=A /\left(A^{1 \times 3} J_{21}\right)$, and $L_{3}=A /\left(A^{1 \times 3} J_{31}\right)$, where $J_{i 1}$ is the first matrix of the $i^{\text {th }}$ entry of $J$. Moreover, the injective $A$-homomorphism $\theta_{i}: L_{i} \longrightarrow M$ is defined by $\theta_{i}\left(\rho_{i}^{\prime}(\lambda)\right)=\pi\left(\lambda J_{i 2}\right)$, where $J_{i 2}$ is the second matrix in the $i^{\text {th }}$ entry of $J$ and $\rho_{i}^{\prime}$ is the canonical projection onto $L_{i}$. We find $L_{1} \cong M_{1}, L_{2} \cong M_{2}$, and $L_{3} \cong M_{3}$. Let us check again that the $A$-homomorphisms $\theta_{i}$ 's are injective:

$>\operatorname{seq}(\operatorname{Test} \operatorname{Inj}(J[i][1], R, J[i][2], A), i=1 \ldots 3)$

true, true, true

Let us compute a finite presentation of the $A$-module $M_{i} / M_{i+1}$ for $i=1,2,3$.

$>\mathrm{F}:=\operatorname{PureFactors}(\mathrm{R}, \mathrm{A})$;

$$
F:=\left[[1],\left[x^{2}-z\right],\left[\begin{array}{c}
y \\
x-1+z
\end{array}\right],\left[\begin{array}{c}
y-1 \\
z+1 \\
x-1
\end{array}\right]\right]
$$

We obtain $M / M_{1}=A /\left(A F_{1}\right)=0$, i.e., $M=M_{1}=t(M), M_{1} / M_{2}=A /\left(A F_{2}\right), M_{2} / M_{3}=$ $A /\left(A^{1 \times 2} F_{3}\right)$, and $M_{3}=A /\left(A^{1 \times 3} F_{3}\right)$, where $F_{i}$ is the $i^{\text {th }}$ matrix of $F$.

Let us check again that the $A$-modules $M_{i} / M_{i+1}$ 's are either 0 or $i$-pure.

$>\operatorname{map}($ IsPure, $, \mathrm{F}, \mathrm{A})$;

$$
[\infty, 1,2,3]
$$

We find again that $M / M_{1}=0$ and $M_{1} / M_{2}$ (resp., $M_{2} / M_{3}$ and $M_{3}$ ) is 1 pure (resp., 2 and 3 pure).

Let us now compute a presentation of the $A$-module $M$ based on the grade filtration of $M$.

$>\mathrm{P}:=$ PurePresentation $(\mathrm{R}, \mathrm{A})$;

$$
\begin{gathered}
P:=\left[\begin{array}{c}
x^{3}+x^{2} y+x^{2} z-x^{2}-x z-y z-z^{2}+z \\
x^{2} y z+x^{2} y-y z^{2}-y z \\
x^{2} y^{2}-x^{2} y-y^{2} z+y z
\end{array}\right],\left[\begin{array}{cccc}
1 & 0 & 0 & 0 \\
0 & x^{2}-z & -1 & 0 \\
0 & 0 & y & -1 \\
0 & 0 & x-1+z & 1 \\
0 & 0 & 0 & y-1 \\
0 & 0 & 0 & z+1 \\
0 & 0 & 0 & x-1
\end{array}\right], \\
\left.\left[\begin{array}{llll}
1 & 1 & 0 & 0
\end{array}\right],\left[\begin{array}{c}
0 \\
1 \\
x^{2}-z \\
x^{2} y-y z
\end{array}\right]\right]
\end{gathered}
$$

We obtain $M=A /\left(A^{1 \times 3} P_{1}\right) \cong \bar{M}=A^{1 \times 4} /\left(A^{1 \times 7} P_{2}\right)$, where $P_{i}$ is the $i^{\text {th }}$ matrix of $P$. If $\bar{\pi}$ is the canonical projection onto $\bar{M}$, then $\varphi: M \longrightarrow \bar{M}$ defined by $\varphi(\pi(\lambda))=\bar{\pi}\left(\lambda P_{3}\right)$ is an isomorphism, whose inverse $\varphi^{-1}: \bar{M} \longrightarrow M$ is $\varphi^{-1}(\bar{\pi}(\mu))=\pi\left(\mu P_{4}\right)$.

Let us check that $\varphi$ is an isomorphism and $\varphi^{-1}$ is defined by $P_{4}$. 
$>$ TestIso(P[1],P[2], $\mathrm{P}[3], \mathrm{A})$;

$>\operatorname{TestIso}(\mathrm{P}[2], \mathrm{P}[1], \mathrm{P}[4], \mathrm{A})$;

true

true

Since $M_{1}=t(M)=M$, we can simply compute a new presentation of $M$ based on the grade filtration of $t(M)$.

$>Q:=$ PurePresentationOfTorsionSubmodule_R(R,A);

$Q:=\left[\left[\begin{array}{ccc}x^{2}-z & -1 & 0 \\ 0 & y & -1 \\ 0 & x-1+z & 1 \\ 0 & 0 & y-1 \\ 0 & 0 & z+1 \\ 0 & 0 & x-1\end{array}\right],\left[\begin{array}{c}x^{3}+x^{2} y+x^{2} z-x^{2}-x z-y z-z^{2}+z \\ x^{2} y z+x^{2} y-y z^{2}-y z \\ x^{2} y^{2}-x^{2} y-y^{2} z+y z\end{array}\right],\left[\begin{array}{c}1 \\ x^{2}-z \\ x^{2} y-y z\end{array}\right]\right]$

We get $M_{1}=M=A /\left(A^{1 \times 3} Q_{2}\right) \cong \bar{M}_{1}=A^{1 \times 3} /\left(A^{1 \times 6} Q_{1}\right)$, where this $A$-isomorphism is defined by the matrix of $Q_{3}$.

Finally, let us check again that $Q_{3}$ defines an isomorphism from $\bar{M}_{1}$ to $M$.

$>\operatorname{TestIso}(\mathrm{Q}[1], \mathrm{Q}[2], \mathrm{Q}[3], \mathrm{A})$;

true

Example 14. Let us consider the commutative polynomial ring $A=\mathbb{Q}\left[x_{1}, x_{2}, x_{3}, x_{4}\right]$

$>A:=$ DefineOreAlgebra (diff $=[x[1], \mathrm{s} 1]$, diff $=[x[2], s 2]$, diff $=[x[3], s 3], \operatorname{diff}=$

$>[\mathrm{x}[4], \mathrm{s} 4]$, polynom $=[\mathrm{s} 1, \mathrm{~s} 2, \mathrm{~s} 3, \mathrm{~s} 4])$ :

and the matrix $R \in A^{1 \times 3}$ defined by:

$>\mathrm{R}:=\operatorname{evalm}\left(\left[\left[\mathrm{x}[1]^{\wedge} 3\right],\left[\mathrm{x}[2]^{\wedge} 3\right],\left[\left(\mathrm{x}[1]^{\wedge} 2+\mathrm{x}[2]^{\wedge} 2\right) * \mathrm{x}[4]+\mathrm{x}[1] * \mathrm{x}[2] * \mathrm{x}[3]\right]\right]\right)$;

$$
R:=\left[\begin{array}{c}
x_{1}{ }^{3} \\
x_{2}{ }^{3} \\
\left(x_{1}^{2}+x_{2}^{2}\right) x_{4}+x_{1} x_{2} x_{3}
\end{array}\right]
$$

Let us consider the $A$-module $M=A / I$, where $I=A^{1 \times 3} R$ is the ideal of $A$ generated by the three entries of $R$, first considered in F. S. Macaulay, "The Algebraic Theory of Modular Systems", Cambridge 1994 (first published in 1916), p. 44.

Let us first try to solve the polynomial system defined by $I$ using the Maple command solve:

$>$ solve (convert $(\mathrm{R}$, set $))$;

$$
\left\{x_{1}=0, x_{2}=0, x_{3}=x_{3}, x_{4}=x_{4}\right\}
$$

According to Maple, the affine algebraic variety $V(I)$ defined by $I$ is the 2-dimensional algebraic variety $\left(x_{1}=0, x_{2}=0, x_{3}=x_{3}, x_{4}=x_{4}\right)$. In particular, if this result is correct, then $V(I)$ would be an equidimensional affine algebraic variety. 
Let us compute the grade filtration of $M$.

$>\mathrm{G}:=$ GradeFiltrationByGenerators $(\mathrm{R}, \mathrm{A})$;

$$
G:=\left[[[1], 1],[[1], 2],\left[\left[\begin{array}{c}
x_{2} x_{1}{ }^{2} \\
-x_{1} x_{2}{ }^{2} \\
x_{1} x_{2}{ }^{2} \\
-x_{2} x_{1}{ }^{2}
\end{array}\right], 3\right],\left[\left[-x_{1}^{2} x_{2}^{2}\right], 4\right]\right]
$$

If $\pi: A \longrightarrow M$ is the canonical projection onto $M$ and $u=\pi(1)$ the generator of $M$, then $M_{1}=A u=M, M_{2}=A u=M, M_{3}=A\left(x_{2} x_{1}^{2}\right) u+A\left(x_{1} x_{2}^{2}\right) u, M_{4}=A\left(x_{1}^{2} x_{2}^{2}\right) u$, and $M_{5}=0$. Hence, the command solve does not compute the whole solution set of the polynomial system defined by $I$. In particular, $V(I)$ is not an equidimensional affine algebraic variety.

Let us now compute a finite presentation of the $A$-module $M_{i} / M_{i+1}$ for $i=0, \ldots, 4$.

$>\mathrm{F}:=$ PureFactors $(\mathrm{R}, \mathrm{A})$;

$$
F:=\left[[1],[1],\left[\begin{array}{c}
x_{1}{ }^{3} \\
x_{2}{ }^{3} \\
x_{1} x_{2}{ }^{2} \\
x_{2} x_{1}{ }^{2} \\
x_{4} x_{1}{ }^{2}+x_{4} x_{2}{ }^{2}+x_{1} x_{2} x_{3}
\end{array}\right],\left[\begin{array}{cc}
0 & -x_{2} \\
x_{2} & 0 \\
-x_{4} & -x_{3} \\
0 & -x_{1} \\
x_{1} & 0 \\
-x_{3} & -x_{4} \\
x_{3}{ }^{2}-x_{4}{ }^{2} & 0
\end{array}\right],\left[\begin{array}{c}
x_{1} \\
x_{2} \\
x_{3} \\
x_{4}
\end{array}\right]\right]
$$

We get $M / M_{1}=A /\left(A F_{1}\right)=0, M_{1} / M_{2}=A /\left(A F_{2}\right)=0, M_{2} / M_{3}=A /\left(A^{1 \times 5} F_{3}\right), M_{3} / M_{4}=$ $A^{1 \times 2} /\left(A^{1 \times 7} F_{4}\right)$, and $M_{4}=A /\left(A^{1 \times 4} F_{5}\right)$.

Let us now check that the $A$-modules $M_{i} / M_{i+1}$ 's are either 0 or $i$-pure.

$>\operatorname{map}($ IsPure, F, A);

$$
[\infty, \infty, 2,3,4]
$$

Let us compute a new presentation of the $A$-module $M$ based on the grade filtration of $M$.

$>\mathrm{P}:=$ PurePresentation $(\mathrm{R}, \mathrm{A})$ :

We obtain that the $A$-module $M$ finitely presented by the matrix

$>\mathrm{P}[1]$;

$$
\left[\begin{array}{c}
x_{1}{ }^{3} \\
x_{2}{ }^{3} \\
\left(x_{1}{ }^{2}+x_{2}{ }^{2}\right) x_{4}+x_{1} x_{2} x_{3}
\end{array}\right]
$$

is isomorphic to the $A$-module $\bar{M}$ finitely presented by the matrix

$>\mathrm{P}[2]$; 


$\left[\begin{array}{cccccc}1 & 0 & 0 & 0 & 0 & 0 \\ 0 & 1 & 0 & 0 & 0 & 0 \\ 0 & 0 & x_{1}{ }^{3} & 0 & 0 & 0 \\ 0 & 0 & x_{2}{ }^{3} & 0 & 0 & 0 \\ 0 & 0 & x_{1} x_{2}{ }^{2} & 0 & -1 & 0 \\ 0 & 0 & x_{1}{ }^{2} x_{2} & -1 & 0 & 0 \\ 0 & 0 & x_{4} x_{1}{ }^{2}+x_{4} x_{2}{ }^{2} x_{1} x_{2} x_{3} & 0 & 0 & 0 \\ 0 & 0 & 0 & 0 & -x_{2} & 0 \\ 0 & 0 & 0 & x_{2} & 0 & -1 \\ 0 & 0 & 0 & -x_{4} & -x_{3} & 0 \\ 0 & 0 & 0 & 0 & -x_{1} & 1 \\ 0 & 0 & 0 & x_{1} & 0 & 0 \\ 0 & 0 & 0 & -x_{3} & -x_{4} & 0 \\ 0 & 0 & 0 & x_{3}^{2}-x_{4}{ }^{2} & 0 & 0 \\ 0 & 0 & 0 & 0 & 0 & x_{1} \\ 0 & 0 & 0 & 0 & 0 & x_{2} \\ 0 & 0 & 0 & 0 & 0 & x_{3} \\ 0 & 0 & 0 & 0 & 0 & x_{4}\end{array}\right]$

i.e., $M \cong \bar{M}=A^{1 \times 6} /\left(A^{1 \times 18} P_{2}\right)$. Moreover, $\varphi: M \longrightarrow \bar{M}$ defined by $\varphi(\pi(\lambda))=\bar{\pi}\left(\lambda P_{3}\right)$, where the matrix $P_{3}$ is given by

$>\mathrm{P}[3]$;

$$
\left[\begin{array}{llllll}
1 & 1 & -1 & 0 & 0 & 0
\end{array}\right]
$$

and $\bar{\pi}: A^{1 \times 6} \longrightarrow \bar{M}$ is the canonical projection onto $\bar{M}$, is an $A$-isomorphism. Its inverse $\varphi^{-1}: \bar{M} \longrightarrow A^{1 \times 6}$ is defined by $\varphi^{-1}(\bar{\pi}(\mu))=\pi\left(\mu P_{4}\right)$, where the matrix $P_{4}$ is defined by:

\section{$>\mathrm{P}[4]$;}

$$
\left[\begin{array}{c}
0 \\
0 \\
-1 \\
-x_{1}{ }^{2} x_{2}-x_{2}{ }^{3} \\
-x_{1} x_{2}{ }^{2} \\
-x_{1}{ }^{2} x_{2}{ }^{2}-x_{2}{ }^{4}
\end{array}\right]
$$

Finally, let us check again that $\varphi$ is an isomorphism

$>$ TestIso(P[1], $P[2], P[3], A)$;

$$
\text { true }
$$


and $\varphi^{-1}$ is defined by matrix $P_{4}$.

$>$ TestIso(P[2], P[1], P[4], A);

true

\subsection{Integration of linear PD systems}

Example 15. Let $A$ be the ring of PD operators in $d x=\frac{\partial}{\partial x}$ and $d t=\frac{\partial}{\partial t}$ with coefficients in $\mathbb{Q}[x, t]$

$>A:=$ DefineOreAlgebra $(\operatorname{diff}=[\mathrm{dx}, \mathrm{x}], \operatorname{diff}=[\mathrm{dt}, \mathrm{t}]$, polynom $=[\mathrm{x}, \mathrm{t}])$ :

and the matrix $R \in A^{1 \times 2}$ of PD operators defined by:

$>\mathrm{R}:=\operatorname{evalm}\left(\left[\left[\mathrm{dt}^{\wedge} 2 *(\mathrm{dx}-\mathrm{dt})\right],[\mathrm{dt} * \mathrm{dx} *(\mathrm{dx}-\mathrm{dt})]\right]\right)$;

$$
\left[\begin{array}{c}
d t^{2}(d x-d t) \\
d t d x(d x-d t)
\end{array}\right]
$$

The corresponding linear system $R y(t, x)=0$ is defined by the following equations:

$>\operatorname{Eqs}:=\operatorname{map}(\mathrm{a}->\mathrm{a}=0, \operatorname{convert}(\operatorname{ApplyMatrix}(\mathrm{R},[\mathrm{y}(\mathrm{t}, \mathrm{x})], \mathrm{A})$, set $))$;

$$
E q s:=\left\{-\frac{\partial^{3}}{\partial x \partial t^{2}} y(t, x)+\frac{\partial^{3}}{\partial x^{2} \partial t} y(t, x)=0, \frac{\partial^{3}}{\partial x \partial t^{2}} y(t, x)-\frac{\partial^{3}}{\partial t^{3}} y(t, x)=0\right\}
$$

Let us use the Maple command pdsolve to integrate the above linear PD system.

$>$ st:=time(): sol:=pdsolve(Eqs, $y(t, x))$; time()-st;

Error, (in combine/power) too many levels of recursion 28.679

Maple cannot solve the linear PD system due to bugs!

Let us now study the grade filtration of the $A$-module $M=A /\left(A^{1 \times 2} R\right)$.

$>\mathrm{G}:=$ GradeFiltrationByGenerators $(\mathrm{R}, \mathrm{A}, \mathrm{opt})$;

$$
G:=\left[\left[\left[\begin{array}{c}
d t^{2} d x-d t^{3} \\
-d t^{3}+d t d x^{2}
\end{array}\right],[1], 1\right],\left[\left[\begin{array}{c}
d t \\
d x
\end{array}\right],\left[-d t d x+d t^{2}\right], 2\right]\right]
$$

If $\pi: A \longrightarrow M$ be the canonical projection onto $M$ and $u=\pi(1)$ the generator of $A$, then $M_{1}=A u=A /\left(A^{1 \times 2} R\right)=M, M_{2}=\left(-d t d x+d t^{2}\right) u \cong A /(A d t+A d x)$, and $M_{3}=0$.

Let us now compute a finite presentation of the $A$-module $M_{i} / M_{i+1}$ for $i=0,1,2$.

$>\mathrm{F}:=\operatorname{PureFactors}(\mathrm{R}, \mathrm{A})$;

$$
F:=\left[[1],\left[d t d x-d t^{2}\right],\left[\begin{array}{c}
d t \\
d x
\end{array}\right]\right]
$$

We obtain $M / M_{1}=A /\left(A F_{1}\right), M_{1} / M_{2}=A /\left(A F_{2}\right)=0$, and $M_{2}=A /\left(A^{1 \times 2} F_{3}\right)$, where $F_{i}$ is the $i^{\text {th }}$ matrix of $F$.

Let us check whether or not the $A$-module $M_{i} / M_{i+1}$ is either 0 or $i$-pure for $i=0,1,2$.

$>\operatorname{map}($ IsPure, $, \mathrm{F}, \mathrm{A})$; 
$[\infty, 1,2]$

Let us now compute a finite presentation of $M$ based on the grade filtration of $M$.

$>\mathrm{P}:=$ PurePresentation $(\mathrm{R}, \mathrm{A})$;

$$
P:=\left[\left[\begin{array}{c}
d t^{2}(d x-d t) \\
d t d x(d x-d t)
\end{array}\right],\left[\begin{array}{ccc}
1 & -1 & 0 \\
0 & d t d x-d t^{2} & 1 \\
0 & 0 & d t \\
0 & 0 & d x
\end{array}\right],\left[\begin{array}{lll}
0 & 1 & 0
\end{array}\right],\left[\begin{array}{c}
1 \\
1 \\
-d t d x+d t^{2}
\end{array}\right]\right]
$$

We obtain $M=A /\left(A^{1 \times 2} P_{1}\right) \cong \bar{M}=A^{1 \times 3} /\left(A^{1 \times 4} P_{2}\right)$, where $P_{i}$ is the $i^{\text {th }}$ matrix of $P$. Moreover, the $A$-isomorphism $\varphi: M \longrightarrow \bar{M}$ is defined by $\varphi(\pi(\lambda))=\bar{\pi}\left(\lambda P_{3}\right)$, where $\bar{\pi}: A^{1 \times 3} \longrightarrow \bar{M}$ is the canonical projection onto $\bar{M}$. Finally, $\varphi^{-1}: \bar{M} \longrightarrow M$ is defined by $\varphi^{-1}(\bar{\pi}(\mu))=\pi\left(\mu P_{4}\right)$.

Let us now check again that $\varphi$ is an isomorphism and $\varphi^{-1}$ is defined by $P_{4}$.

$>$ TestIso(P[1], $\mathrm{P}[2], \mathrm{P}[3], \mathrm{A})$;

$$
>\text { TestIso(P[2],P[1],P[4], A); } \quad \text { true }
$$

true

Let us now try to integrate the above linear PD system by using its equivalence form $P_{2} z=0$.

$$
\begin{aligned}
& >\quad i v:=o p(A[3]) \text {; } \\
& i v:=x, t \\
& >\text { Eqs: }=\operatorname{map}(\mathrm{a}->\mathrm{a}=0 \text {, convert (convert (ApplyMatrix (P [2], [zeta[1] (iv), zeta [2] (iv), } \\
& >\operatorname{zeta}[3](\text { iv) }], A) \text {, vector), set)); } \\
& \text { Eqs }:= \\
& \left\{\zeta_{1}(x, t)-\zeta_{2}(x, t)=0,-\frac{\partial^{2}}{\partial t^{2}} \zeta_{2}(x, t)+\frac{\partial^{2}}{\partial x \partial t} \zeta_{2}(x, t)+\zeta_{3}(x, t)=0, \frac{\partial}{\partial t} \zeta_{3}(x, t)=0, \frac{\partial}{\partial x} \zeta_{3}(x, t)=0\right\}
\end{aligned}
$$

We obtain

$$
\begin{aligned}
& >\text { st:=time(): z:=pdsolve(Eqs, zeta[1] (iv), zeta[2] (iv), zeta[3] (iv)); } \\
& >\text { time }()-\text { st; } \\
& z:=\left\{\zeta_{1}(x, t)={ }_{-} F 1(x)+{ }_{-} F 2(x+t)-1 / 2{ }_{-} C 1 x(x+2 t),\right. \\
& \left.\zeta_{2}(x, t)={ }_{-} F 1(x)+{ }_{-} F 2(x+t)-1 / 2{ }_{-} C 1 x(x+2 t), \zeta_{3}(x, t)={ }_{-} C 1\right\} \\
& 0.019
\end{aligned}
$$

i.e., the general solution $Z$ of $P_{2} z=0$ is defined by:

$$
\begin{gathered}
>\mathrm{Z}:=\operatorname{evalm}([\operatorname{rhs}(\operatorname{sol}[1])],[\operatorname{rhs}(\operatorname{sol}[2])],[\operatorname{rhs}(\operatorname{sol}[3])]]) ; \\
Z:=\left[\begin{array}{c}
-F 1(x)+{ }_{-} F 2(x+t)-1 / 2{ }_{-} C 1 x(x+2 t) \\
{ }_{-} F 1(x)+{ }_{-} F 2(x+t)-1 / 2{ }_{-} C 1 x(x+2 t) \\
{ }_{-} C 1
\end{array}\right]
\end{gathered}
$$

Let us check again that $Z$ is a solution of the linear $\mathrm{PD}$ system $P_{2} Z=0$ :

$>$ ApplyMatrix $(\mathrm{P}[2], \mathrm{Z}, \mathrm{A})$; 


$$
\left[\begin{array}{l}
0 \\
0 \\
0 \\
0
\end{array}\right]
$$

Now, the solution of the linear $\mathrm{PD}$ system $R y=0$ is defined by $y=P_{3} z$, i.e.:

$>\mathrm{y}:=\operatorname{ApplyMatrix}(\mathrm{P}[3], \mathrm{Z}, \mathrm{A})$;

$$
y:=\left[{ }_{-} F 1(x)+{ }_{-} F 2(x+t)-1 / 2{ }_{-} C 1 x^{2}{ }_{-} C 1 x t\right]
$$

Let us check that $y$ is a solution of the linear PD system $R y=0$.

$>$ ApplyMatrix $(\mathrm{R}, \mathrm{y}, \mathrm{A})$;

$$
\left[\begin{array}{l}
0 \\
0
\end{array}\right]
$$

This result can be directly obtained by the command IntegrationOfDModule.

$>$ sol:=IntegrationOfDModule (R,A);

$$
\text { sol }:=\left[{ }_{-} F 1(x)+{ }_{-} F 2(x+t)-1 / 2{ }_{-} C 1 x^{2}-{ }_{-} C 1 x t\right]
$$

$>$ ApplyMatrix $(\mathrm{R}, \mathrm{sol}, \mathrm{A})$;

$$
\left[\begin{array}{l}
0 \\
0
\end{array}\right]
$$

Example 16. Let $A$ be the ring of $\mathrm{PD}$ operators in $d x=\frac{\partial}{\partial x}$ and $d t=\frac{\partial}{\partial t}$ with coefficients in $\mathbb{Q}[x, t]$

$>A:=$ DefineOreAlgebra $(\operatorname{diff}=[\mathrm{dx}, \mathrm{x}], \operatorname{diff}=[\mathrm{dt}, \mathrm{t}]$, polynom $=[\mathrm{x}, \mathrm{t}])$ :

and the matrix $R \in A^{1 \times 2}$ of PD operators defined by:

$>\mathrm{R}:=\operatorname{evalm}\left(\left[\left[\mathrm{dx}^{\wedge} 2 *(\mathrm{dt}-\mathrm{dx})\right],\left[\mathrm{dt}^{\wedge} 2 *(\mathrm{dt}-\mathrm{dx})\right]\right]\right)$;

$$
R:=\left[\begin{array}{l}
d x^{2}(d t-d x) \\
d t^{2}(d t-d x)
\end{array}\right]
$$

Let us use the Maple command pdsolve to integrate the linear PD system $R y(x, t)=0$, i.e.,

$>\quad i v:=o p(A[3])$;

$$
i v:=x, t
$$

the linear system of PD equations defined by:

$>\operatorname{Eqs}:=\operatorname{map}(\mathrm{a}->\mathrm{a}=0, \operatorname{convert}(\operatorname{ApplyMatrix}(\mathrm{R},[\mathrm{y}(\mathrm{iv})], \mathrm{A})$, set $))$;

$$
E q s:=\left\{-\frac{\partial^{3}}{\partial x \partial t^{2}} y(x, t)+\frac{\partial^{3}}{\partial t^{3}} y(x, t)=0, \frac{\partial^{3}}{\partial x^{2} \partial t} y(x, t)-\frac{\partial^{3}}{\partial x^{3}} y(x, t)=0\right\}
$$

Maple cannot integrate the linear PD system due to bugs!

$$
\text { > st:=time(): sol:=pdsolve(Eqs,y(iv)); time()-st; }
$$


Error, (in dchange/funcs) not implemented case of many integrals w.r.t the same variable inside a multiple integral

0.698

Let us now compute the grade filtration of the $A$-module $M=A /\left(A^{1 \times 2} R\right)$.

$>\mathrm{G}:=$ GradeFiltrationByGenerators $(\mathrm{R}, \mathrm{A})$;

$$
G:=\left[\left[\left[\begin{array}{c}
-d t^{3}+d t^{2} d x \\
-d x^{2} d t+d x^{3}
\end{array}\right],[1], 1\right],\left[\left[\begin{array}{c}
d t^{2} \\
d x^{2}
\end{array}\right],[d t-d x], 2\right]\right]
$$

If $\pi: A \longrightarrow M$ is the canonical projection onto $M$ and $u=\pi(1)$ the generator of $M$, then $M_{1}=A u=M=A /\left(A^{1 \times 2} R\right), M_{2}=A(d t-d x) u \cong A /\left(A d t^{2}+A d x^{2}\right)$, and $M_{3}=0$.

Let us compute a finite presentation of the $A$-module $M_{i} / M_{i+1}$ for $i=0,1,2$.

$>\mathrm{F}:=$ PureFactors $(\mathrm{R}, \mathrm{A})$;

$$
\left.F:=[1],[d x-d t],\left[\begin{array}{c}
d t^{2} \\
d x^{2}
\end{array}\right]\right]
$$

We get $M / M_{1}=A /\left(A F_{1}\right)=0, M_{1} / M_{2}=A /\left(A F_{2}\right)$, and $M_{2}=A /\left(A^{1 \times 2} F_{3}\right)$, where $F_{i}$ is the $i^{\text {th }}$ matrix of $F$.

Let us check again that $M_{i} / M_{i+1}$ is either 0 or $i$-pure for $i=0,1,2$.

$>\operatorname{map}($ IsPure, F, A);

$$
[\infty, 1,2]
$$

Since $M=M_{1}=t(M), M$ is a torsion $A$-module. Let us compute a finite presentation of $M$ based on the grade filtration of $M_{1}$.

$>P:=$ PurePresentationOfTorsionSubmodule $(\mathrm{R}, \mathrm{A})$;

$$
P:=\left[\left[\begin{array}{cc}
d x-d t & 1 \\
0 & d t^{2} \\
0 & d x^{2}
\end{array}\right],\left[\begin{array}{c}
d x^{2}(d t-d x) \\
d t^{2}(d t-d x)
\end{array}\right],\left[\begin{array}{c}
1 \\
d t-d x
\end{array}\right]\right]
$$

We get $L=A^{1 \times 2} /\left(A^{1 \times 3} P_{1}\right) \subseteq M=A /\left(A^{1 \times 2} P_{2}\right)$. The injection $\iota: L \longrightarrow M$ is defined by $\iota(\kappa(\mu))=\pi\left(\mu P_{3}\right)$, where $P_{i}$ is the $i^{\text {th }}$ matrix of $P$ and $\kappa: A^{1 \times 2} \longrightarrow L$ is the canonical projection onto $L$. Let us check again that $\iota$ is an injection.

$>\operatorname{TestInj}(\mathrm{P}[1], \mathrm{P}[2], \mathrm{P}[3], \mathrm{A})$;

$$
\text { true }
$$

Since $M_{1}=M, \iota$ is also an isomorphism, which can be easily check again.

$>$ TestIso(P[1],P[2], P[3],A);

true

The inverse $\iota^{-1}: M \longrightarrow L$ of $\iota$ can then be computed as follows.

$>\mathrm{T}:=\operatorname{InverseMorphism}(\mathrm{P}[1], \mathrm{P}[2], \mathrm{P}[3], \mathrm{A})$;

$$
T:=\left[\left[\begin{array}{ll}
1 & 0
\end{array}\right],\left[\begin{array}{ccc}
-d x^{2} & 0 & 1 \\
-d t^{2} & 1 & 0
\end{array}\right]\right]
$$

$\mathrm{RR} \mathrm{n}^{\circ} 7769$ 
Thus, $\iota^{-1}: M \longrightarrow L$ is defined by $\iota^{-1}(\pi(\lambda))=\kappa\left(\lambda T_{1}\right)$, where $T_{1}$ is the first matrix of $T$. Let us check again that the $A$-homomorphism defined by $T_{1}$ defined an isomorphism.

$>$ TestIso(P[2],P[1], T[1], A);

true

Let us try to integrate $P_{1} z=0$ using the command IntegrationOfTorsionDSubmodule.

$>\mathrm{z}:=$ IntegrationOfTorsionDSubmodule $(\mathrm{R}, \mathrm{A}) ;$
$z:=\left[\begin{array}{c}-1 / 6 x^{3}{ }_{-} C 3+\left(-1 / 2{ }_{-} C 1-1 / 2{ }_{-} C 3 t-1 / 2{ }_{-} C 4\right) x^{2}+\left({ }_{-} C 1 t-{ }_{-} C 2\right) x+{ }_{-} F 1(t+x) \\ \left(x_{-} C 3+{ }_{-} C 1\right) t+x_{-} C 4+{ }_{-} C 2\end{array}\right]$

Let us check again that $z$ is a solution of $P_{1} z=0$.

$>\operatorname{ApplyMatrix}(P[1], z, A)$;

$$
\left[\begin{array}{l}
0 \\
0 \\
0
\end{array}\right]
$$

Then, $y=T_{1} z$, namely,

$$
\begin{aligned}
> & \mathrm{y}:=\operatorname{ApplyMatrix}(\mathrm{T}[1], \mathrm{z}, \mathrm{A}) ; \\
y & :=\left[-1 / 6 t^{3}{ }_{-} C 3-1 / 2 t^{2}{ }_{-} C 3 x-1 / 2 t^{2}{ }_{-} C 4-1 / 2{ }_{-} C 1 t^{2}-{ }_{-} C 1 t x-{ }_{-} C 2 t+{ }_{-} F 1(x+t)\right]
\end{aligned}
$$

is a solution of the linear PD system $R y=0$.

$>\operatorname{ApplyMatrix}(\mathrm{R}, \mathrm{y}, \mathrm{A})$;

$$
\left[\begin{array}{l}
0 \\
0
\end{array}\right]
$$

This last result can be directly be obtained using the command IntegrationOfDModule.

$$
\begin{aligned}
& >\mathrm{y}:=\text { IntegrationOfDModule }(\mathrm{R}, \mathrm{A}) ; \\
& y:=\left[-1 / 6 x^{3}{ }_{-} C 3-1 / 2 x^{2}{ }_{-} C 3 t-1 / 2 x^{2}{ }_{-} C 4-1 / 2{ }_{-} C 1 x^{2}-{ }_{-} C 1 x t-{ }_{-} C 2 x+{ }_{-} F 1(t+x)\right] \\
& >\quad \text { ApplyMatrix }(\mathrm{R}, \mathrm{y}, \mathrm{A}) ; \\
& \\
& {\left[\begin{array}{l}
0 \\
0
\end{array}\right]}
\end{aligned}
$$

Example 17. Let $A$ be the ring of PD operators in $d_{1}=\frac{\partial}{\partial x_{1}}, d_{2}=\frac{\partial}{\partial x_{2}}$, and $d_{3}=\frac{\partial}{\partial x_{3}}$ with coefficients in the ring $\mathbb{Q}\left[x_{1}, x_{2}, x_{3}\right]$

$>A:=$ DefineOreAlgebra $(\operatorname{diff}=[d[1], x[1]], \operatorname{diff}=[d[2], x[2]]$, polynom $=[x[1], x[2]])$ : and the matrix $R \in A^{1 \times 2}$ of $\mathrm{PD}$ operators defined by:

$$
\begin{gathered}
>\quad \mathrm{R}:=\operatorname{evalm}([[\mathrm{d}[1] \sim 2+\mathrm{d}[2] * \mathrm{~d}[1]-(\mathrm{x}[1]+\mathrm{x}[2]) * \mathrm{~d}[1]-1],[\mathrm{d}[2] \wedge 2+\mathrm{d}[2] * \mathrm{~d}[1] \\
>-(\mathrm{x}[1]+\mathrm{x}[2]) * \mathrm{~d}[2]-1]]) ; \\
R:=\left[\begin{array}{c}
d_{1}{ }^{2}+d_{2} d_{1}-\left(x_{1}+x_{2}\right) d_{1}-1 \\
d_{2}{ }^{2}+d_{2} d_{1}-\left(x_{1}+x_{2}\right) d_{2}-1
\end{array}\right]
\end{gathered}
$$


Let us try to integrate the linear PD system $R \eta=0$ defined by

$>\quad i v:=o p(A[3])$;

$$
i v:=x_{1}, x_{2}
$$

$>$ Eqs $:=\operatorname{map}(a->a=0, \operatorname{convert}(\operatorname{convert}(\operatorname{ApplyMatrix}(R,[$ eta(iv)], A), vector), set));

$$
\text { Eqs := }
$$

$$
\begin{aligned}
& \left\{-\eta\left(x_{1}, x_{2}\right)-\left(\frac{\partial}{\partial x_{1}} \eta\left(x_{1}, x_{2}\right)\right) x_{1}-\left(\frac{\partial}{\partial x_{1}} \eta\left(x_{1}, x_{2}\right)\right) x_{2}+\frac{\partial^{2}}{\partial x_{2} \partial x_{1}} \eta\left(x_{1}, x_{2}\right)+\frac{\partial^{2}}{\partial x_{1}{ }^{2}} \eta\left(x_{1}, x_{2}\right)=0,\right. \\
& \left.-\eta\left(x_{1}, x_{2}\right)-\left(\frac{\partial}{\partial x_{2}} \eta\left(x_{1}, x_{2}\right)\right) x_{1}-\left(\frac{\partial}{\partial x_{2}} \eta\left(x_{1}, x_{2}\right)\right) x_{2}+\frac{\partial^{2}}{\partial x_{2} \partial x_{1}} \eta\left(x_{1}, x_{2}\right)+\frac{\partial^{2}}{\partial x_{2}{ }^{2}} \eta\left(x_{1}, x_{2}\right)=0\right\}
\end{aligned}
$$

Maple cannot solve the linear PD system since no output is returned:

$>$ eta: $=$ pdsolve(Eqs, eta(iv));

$$
\text { eta }:=
$$

Let us now compute the grade filtration of the left $A$-module $M=A /\left(A^{1 \times 2} R\right)$.

$$
\begin{aligned}
& >\text { GradeFiltrationByGenerators (R,A,opt); } \\
& \left.\left[\left[\begin{array}{c}
d_{2}^{2}+d_{2} d_{1}-d_{2} x_{1}-d_{2} x_{2}-1 \\
d_{2} x_{2}-d_{1} x_{2}+d_{2} x_{1}-d_{1} x_{1}-d_{2}{ }^{2}+d_{1}{ }^{2}
\end{array}\right],[1], 1\right],\left[\left[\begin{array}{c}
d_{2} \\
d_{1}
\end{array}\right],\left[-d_{1}-d_{2}+x_{1}+x_{2}\right], 2\right]\right]
\end{aligned}
$$

If $\pi: A \longrightarrow M=A /\left(A^{1 \times 2} R\right)$ is the canonical projection and $u=\pi(1)$ the generator of $M$, then $M_{1}=A u=M=A /\left(A^{1 \times 2} R\right), M_{2}=A\left(-d_{1}-d_{2}+x_{1}+x_{2}\right) u \cong A /\left(A d_{1}+A d_{2}\right)$, and $M_{3}=0$.

Let us compute a finite presentation of the left $A$-module $M_{i} / M_{i+1}$ for $i=0,1,2$.

$>\mathrm{F}:=$ PureFactors $(\mathrm{R}, \mathrm{A})$;

$$
F:=\left[[1],\left[d_{1}+d_{2}-x_{1}-x_{2}\right],\left[\begin{array}{l}
d_{1} \\
d_{2}
\end{array}\right]\right]
$$

We obtain $M / M_{1}=A /\left(A F_{1}\right)=0, M_{1} / M_{2}=A /\left(A F_{2}\right)$, and $M_{2}=A /\left(A^{1 \times 3} F_{3}\right)$, where $F_{i}$ is the $i^{\text {th }}$ matrix of $F$. Let check whether or not $M_{i} / M_{i+1}$ is 0 or $i$-pure for $i=0,1,2$.

$>\operatorname{map}($ IsPure, $\mathrm{F}, \mathrm{A})$;

$$
[\infty, 1,2]
$$

Let us now compute a new presentation of the left $A$-module $M=A /\left(A^{1 \times 2} R\right)$ based on the grade filtration of $M$.

$$
\begin{aligned}
> & \mathrm{P}:=\text { PurePresentationOfTorsionSubmodule }(\mathrm{R}, \mathrm{A}) \\
P & \left.:=\left[\begin{array}{cc}
d_{1}+d_{2}-x_{1}-x_{2} & 1 \\
0 & d_{1} \\
0 & d_{2}
\end{array}\right],\left[\begin{array}{c}
d_{1}^{2}+d_{2} d_{1}-\left(x_{1}+x_{2}\right) d_{1}-1 \\
d_{2}{ }^{2}+d_{2} d_{1}-\left(x_{1}+x_{2}\right) d_{2}-1
\end{array}\right],\left[\begin{array}{c}
1 \\
-d_{1}-d_{2}+x_{1}+x_{2}
\end{array}\right]\right]
\end{aligned}
$$

We get $L=A^{1 \times 2} /\left(A^{1 \times 3} P_{1}\right) \subseteq M=A /\left(A^{1 \times 2} R\right)$. The injection $\iota: L \longrightarrow M$ is defined by $\iota(\kappa(\mu))=\pi\left(\mu P_{3}\right)$, where $\kappa: A^{1 \times 2} \longrightarrow L$ is the canonical projection onto $L$ and $P_{i}$ is the $i^{\text {th }}$ matrix of $P$. Let us check again that $\iota$ is injective.

$>\operatorname{TestInj}(\mathrm{P}[1], \mathrm{P}[2], \mathrm{P}[3], \mathrm{A})$;

true 
Since $M=M_{1}=t(M), \iota$ is also an isomorphism, which can be easily check again.

$>$ TestIso(P[1],P[2], $\mathrm{P}[3], \mathrm{A})$;

true

The inverse $\iota^{-1}: M \longrightarrow L$ of $\iota$ is then defined by

$>\mathrm{T}:=$ InverseMorphism(P[1], $\mathrm{P}[2], \mathrm{P}[3], \mathrm{A})$;

$$
T:=\left[\left[\begin{array}{cc}
1 & 0
\end{array}\right],\left[\begin{array}{ccc}
d_{1} & -1 & 0 \\
d_{2} & 0 & -1
\end{array}\right]\right]
$$

i.e., $\iota^{-1}(\pi(\lambda))=\kappa\left(\lambda T_{1}\right)$ for all $\lambda \in A$. Let us check again that the $A$-homomorphism defined by $T_{1}$ is an isomorphism.

$>$ TestIso(P[2],P[1], T[1], A);

true

Let us now try to solve the linear PD system $P_{1} z=0$ defined by the following PD equations:

$>$ eqs : $=\operatorname{map}(\mathrm{a}->\mathrm{a}=0$, convert (convert (ApplyMatrix (P[1], [zeta[1] (iv), zeta[2] (iv)],

$>$ A), vector), set));

$$
\begin{gathered}
\text { eqs }:= \\
\left\{-\zeta_{1}\left(x_{1}, x_{2}\right) x_{1}-\zeta_{1}\left(x_{1}, x_{2}\right) x_{2}+\frac{\partial}{\partial x_{1}} \zeta_{1}\left(x_{1}, x_{2}\right)+\frac{\partial}{\partial x_{2}} \zeta_{1}\left(x_{1}, x_{2}\right)+\zeta_{2}\left(x_{1}, x_{2}\right)=0,\right. \\
\left.\frac{\partial}{\partial x_{1}} \zeta_{2}\left(x_{1}, x_{2}\right)=0, \frac{\partial}{\partial x_{2}} \zeta_{2}\left(x_{1}, x_{2}\right)=0\right\}
\end{gathered}
$$

We obtain:

$$
\begin{gathered}
>\mathrm{z}:=\text { pdsolve (eqs, zeta[1] (iv), zeta[2] (iv)) } \\
z:= \\
\left\{\zeta_{1}\left(x_{1}, x_{2}\right)=-1 / 2\left({ }_{-} C 1 \sqrt{\pi} \mathrm{e}^{1 / 4\left(-x_{1}+x_{2}\right)^{2}} \operatorname{erf}\left(1 / 2 x_{1}+1 / 2 x_{2}\right)-2{ }_{-} F 1\left(-x_{1}+x_{2}\right)\right) \mathrm{e}^{x_{1}{ }^{2}+\left(-x_{1}+x_{2}\right) x_{1}},\right. \\
\left.\zeta_{2}\left(x_{1}, x_{2}\right)={ }_{-} C 1\right\}
\end{gathered}
$$

In other words, the vector $Z$ defined by

$$
\begin{aligned}
& >Z:=\operatorname{evalm}([\operatorname{rhs}(\operatorname{sol}[1])],[\operatorname{rhs}(\operatorname{sol}[2])]]) ; \\
& Z:=\left[\begin{array}{c}
-1 / 2\left({ }_{-} C 1 \sqrt{\pi} \mathrm{e}^{1 / 4\left(-x_{1}+x_{2}\right)^{2}} \operatorname{erf}\left(1 / 2 x_{1}+1 / 2 x_{2}\right)-2{ }_{-} F 1\left(-x_{1}+x_{2}\right)\right) \mathrm{e}^{x_{1}{ }^{2}+\left(-x_{1}+x_{2}\right) x_{1}} \\
-C 1
\end{array}\right]
\end{aligned}
$$

is a solution of the linear $\mathrm{PD}$ system $P_{1} Z=0$.

$>\operatorname{ApplyMatrix}(\mathrm{P}[1], \mathrm{Z}, \mathrm{A})$;

$$
\left[\begin{array}{l}
0 \\
0 \\
0
\end{array}\right]
$$

Now, $y=T_{1} Z$, namely,

$>\mathrm{y}:=\operatorname{ApplyMatrix}(\mathrm{T}[1], \mathrm{Z}, \mathrm{A})$; 


$$
y:=\left[-1 / 2\left(-C 1 \sqrt{\pi} \mathrm{e}^{1 / 4\left(x_{1}-x_{2}\right)^{2}} \operatorname{erf}\left(1 / 2 x_{1}+1 / 2 x_{2}\right)-2{ }_{-} F 1\left(-x_{1}+x_{2}\right)\right) \mathrm{e}^{x_{1} x_{2}}\right]
$$

is a solution of the linear PD system $R y=0$.

$>$ ApplyMatrix $(\mathrm{R}, \mathrm{y}, \mathrm{A})$;

$$
\left[\begin{array}{l}
0 \\
0
\end{array}\right]
$$

We can directly integrate $P_{1} z=0$ using the command IntegrationOfTorsionDSubmodule:

$$
\begin{aligned}
& >\mathrm{U}:=\text { IntegrationOfTorsionDSubmodule }(\mathrm{R}, \mathrm{A}) ; \\
& U:=\left[\begin{array}{c}
-1 / 2\left({ }_{-} C 1 \sqrt{\pi} \mathrm{e}^{1 / 4\left(-x_{1}+x_{2}\right)^{2}} \operatorname{erf}\left(1 / 2 x_{1}+1 / 2 x_{2}\right)-2{ }_{-} F 1\left(-x_{1}+x_{2}\right)\right) \mathrm{e}^{x_{1}{ }^{2}+\left(-x_{1}+x_{2}\right) x_{1}} \\
-C 1
\end{array}\right] \\
& >\operatorname{ApplyMatrix}(\mathrm{P}[1], \mathrm{U}, \mathrm{A}) ;
\end{aligned}
$$

$$
\left[\begin{array}{l}
0 \\
0 \\
0
\end{array}\right]
$$

Finally, the linear PD system $R y=0$ can also be directly integrated using the command IntegrationOfDModule.

$>\mathrm{X}:=:=$ IntegrationOfDModule $(\mathrm{R}, \mathrm{A}, \mathrm{a})$;

$$
X:=\left[-1 / 2\left({ }_{-} C 1 \sqrt{\pi} \mathrm{e}^{1 / 4\left(x_{1}-x_{2}\right)^{2}} \operatorname{erf}\left(1 / 2 x_{1}+1 / 2 x_{2}\right)-2{ }_{-} F 1\left(-x_{1}+x_{2}\right)\right) \mathrm{e}^{x_{1} x_{2}}\right]
$$

$>$ ApplyMatrix $(\mathrm{R}, \mathrm{X}, \mathrm{A})$;

$$
\left[\begin{array}{l}
0 \\
0
\end{array}\right]
$$

\section{References}

[1] M. Auslander and M. Bridger. Stable Module Theory, volume 94 of Memoirs of the American Mathematical Society. AMS, 1969.

[2] M. Barakat. Spectral filtrations via generalized morphisms. Technical report, ArXiv, 2009. http://arxiv.org/abs/0904.0240, submitted for publication, version 2 .

[3] M. Barakat. Purity filtration and the fine structure of autonomy. In $19^{\text {th }}$ International Symposium on Mathematical Theory of Networks and Systems (MTNS), Budapest (Hungary), 2010 .

[4] M. Barakat and B. Bremer. Higher extension modules and the Yoneda product. Technical report, ArXiv, 2008. http://arxiv.org/abs/0802.3179, submitted for publication.

[5] M. Barakat and M. Lange-Hegermann. The Sheaves package - A homalg based package for the Abelian category of coherent sheaves over a projective scheme, 2008-2011. (http: //homalg.math.rwth-aachen.de/index.php/unreleased/sheaves). 
[6] M. Barakat and M. Lange-Hegermann. An axiomatic setup for algorithmic homological algebra and an alternative approach to localization. J. Algebra Appl., 10:269-293, 2011. http://arxiv.org/abs/1003.1943).

[7] M. Barakat and A. Quadrat. The AbelianSystems project, 2011. A homalg package (GAP4), in development. http://homalg.math.rwth-aachen.de/,

[8] M. Barakat and D. Robertz. homalg - A meta-package for homological algebra. J. Algebra Appl., 7(3):299-317, 2008. http://homalg.math.rwth-aachen.de/.

[9] J. E. Björk. Rings of Differential Operators. North Holland, 1979.

[10] J. E. Björk. Analytic D-modules and Applications. Kluwer, 1993.

[11] A. Borel. Algebraic D-modules, volume 2 of Perspectives in Mathematics. Academic Press, 1987.

[12] B. Buchberger. An algorithm for finding the basis elements in the residue class ring modulo a zero dimensional polynomial ideal. J. Symbolic Comput., 41:475-511, 2006. PhD Thesis (English translation).

[13] E. Cartan and A. Einstein. Lettres sur le parallélisme absolu. Académie Royale de Belgique, Princeton University Press, 1979.

[14] F. Chyzak, A. Quadrat, and D. Robertz. Effective algorithms for parametrizing linear control systems over Ore algebras. Appl. Algebra Engrg. Comm. Comput., 16:319-376, 2005 .

[15] F. Chyzak, A. Quadrat, and D. Robertz. OreModules: A symbolic package for the study of multidimensional linear systems. In J. Chiasson and J.-J. Loiseau, editors, Applications of Time-Delay Systems, volume 352 of Lecture Notes in Control and Inform. Sci., pages 233-264. Springer, 2007. The OreModules project http://wwwb.math.rwth-aachen. de/OreModules.

[16] T. Cluzeau and A. Quadrat. Factoring and decomposing a class of linear functional systems. Linear Algebra Appl., 428:324-381, 2008.

[17] T. Cluzeau and A. Quadrat. OreMorphisms: A homological algebraic package for factoring and decomposing linear functional systems. In J.-J. Loiseau, W. Michiels, S.I. Niculescu, and R. Sipahi, editors, Topics in Time-Delay Systems: Analysis, Algorithms and Control, volume 388 of Lecture Notes in Control and Inform. Sci., pages 179196. Springer, 2009. The OreMorphisms project http://www-sop.inria.fr/members/ Alban.Quadrat/OreMorphisms/index.html.

[18] T. Cluzeau and A. Quadrat. A constructive version of Fitting's theorem on isomorphisms and equivalences of linear systems. In $7^{\text {th }}$ International Workshop on Multidimensional (nD) Systems, Poitiers (France), 2011.

[19] R. Courant and D. Hilbert. Methods of Mathematical Physics. Wiley Classics Library. Wiley, 1989.

[20] D. Eisenbud, C. Huneke, and W. Vasconcelos. Direct methods for primary decomposition. Invent. Math., 110:207-235, 1992. 
[21] A. Fabiańska and A. Quadrat. Applications of the Quillen-Suslin theorem to multidimensional systems theory. In H. Park and G. Regensburger, editors, Gröbner Bases in Control Theory and Signal Processing, volume 3 of Radon Ser. Comput. Appl. Math., pages 23-106. Walter de Gruyter, 2007. The QuILLENSUSLIN project: http://wwwb.math.rwth-aachen. de/QuillenSuslin.

[22] The GAP Group. GAP - Groups, Algorithms, and Programming, Version 4.4.12, 2008. http://www.gap-system.org.

[23] V. Ginsburg. Characteristic varieties and vanishing cycles. Invent. Math., 84:327-402, 1986.

[24] M. Gromov. Differential Partial Relations. Springer, 1986.

[25] R. Hartshorne. Residue and Duality, volume 20 of Lectures Notes in Math. Springer, 1966.

[26] M. Janet. Sur les systèmes aux dérivées partielles comprenant autant d'équations que de fonctions inconnues. C. R. Acad. Sci. Paris, 172:1637-1639, 1921.

[27] M. Janet. Leçons sur les systèmes d'équations aux dérivées partielles. Cahiers scientifiques IV. Gauthier-Villars, 1929.

[28] J. Johnson. Systems of $n$ partial differential equations in $n$ unknown functions: the conjecture of M. Janet. Trans. Amer. Math. Soc., 242:329-334, 1978.

[29] M. Kashiwara. B-functions and holonomic systems. Invent. Math., 38:33-53, 1976.

[30] M. Kashiwara. Algebraic Study of Systems of Partial Differential Equations, volume 63 of Mémoires of Société Mathématique de France. SMF, 1995. Master Thesis, Tokyo Univ. 1970 (English translation).

[31] V. Levandovskyy. Non-commutative Computer Algebra for Polynomial Algebras: Gröbner Bases, Applications and Implementation. PhD thesis, University of Kaiserslautern, 2005.

[32] T. Levasseur. Complexe bidualisant en algèbre non commutative. In Séminaire DubreuilMalliavin, 1983-1984, volume 1146 of Lecture Notes in Math., pages 270-287. Springer, 1985.

[33] P. Maisonobe and C. Sabbah. D-modules cohérents et holonomes. Travaux en Cours 45. Hermann, 1993.

[34] B. Malgrange. Systèmes différentiels à coefficients constants. Séminaire Bourbaki 1962/63, pages $1-11,1962$.

[35] J. C. McConnell and J. C. Robson. Noncommutative Noetherian Rings. American Mathematical Society, 2000.

[36] U. Oberst. Multidimensional constant linear systems. Acta Appl. Math., 20:1-175, 1990.

[37] U. Oberst. The significance of Gabriel localization for stability and stabilization of multidimensional input/output behaviors. In $19^{\text {th }}$ International Symposium on Mathematical Theory of Networks and Systems (MTNS), Budapest (Hungary), 2010.

[38] J.-F. Pommaret. Partial Differential Control Theory. Kluwer, 2001. 
[39] J.-F. Pommaret. Macaulay inverse systems revisited. J. Symbolic Comput., 46:1049-1069, 2011.

[40] J.-F. Pommaret and A. Quadrat. Algebraic analysis of linear multidimensional control systems. IMA J. Math. Control Inform., 16:275-297, 1999.

[41] A. Quadrat. Extended Bézout identities. In European Control conference (ECC'01), Porto (Portugal), 2001.

[42] A. Quadrat. Purity filtration of 2-dimensional linear systems. In $19^{\text {th }}$ International Symposium on Mathematical Theory of Networks and Systems (MTNS), Budapest (Hungary), 2010.

[43] A. Quadrat. Equidimensional triangularization of multidimensional linear systems. In $7^{\text {th }}$ International Workshop on Multidimensional (nD) Systems, Poitiers (France), 2011.

[44] A. Quadrat. Purity filtration of multidimensional linear systems. In $7^{\text {th }}$ International Workshop on Multidimensional (nD) Systems, Poitiers (France), 2011.

[45] A. Quadrat. The PurityFiltration project, 2011. http://www-sop.inria.fr/members/ Alban.Quadrat/PurityFiltration.html.

[46] A. Quadrat and D. Robertz. On the Baer extension problem for multidimensional linear systems. Technical report, INRIA report 6307, 2007. http://hal.inria.fr/inria-00175272.

[47] A. Quadrat and D. Robertz. Baer's extension problem for multidimensional linear systems. In $18^{\text {th }}$ International Symposium on Mathematical Theory of Networks and Systems (MTNS), Virginia (USA), 2008.

[48] D. Robertz. Janet bases and applications. In M. Rosenkranz and D. Wang, editors, Gröbner Bases in Symbolic Analysis, volume 2 of Radon Ser. Comput. Appl. Math., pages 139-168. Walter de Gruyter, 2007. The JanetOre project: http://wwwb.math.rwth-aachen.de/ $\sim$ daniel/index.html.

[49] J.-E. Roos. Bidualité et structures des foncteurs dérivés de $\lim _{\leftarrow}$ dans la catégorie des modules sur un anneau régulier. C. R. Acad. Sci. Paris, 254:1556-1558, 1962.

[50] J. J. Rotman. An Introduction to Homological Algebra. Springer, second edition, 2009.

[51] D. C. Spencer. Overdetermined systems of linear partial differential equations. Bull. Amer. Math. Soc., 75:179-239, 1969.

[52] J. T. Stafford. Non-holonomic modules over Weyl algebras and enveloping algebras. Invent. Math., 79:619-638, 1984.

[53] J. Wood. Modules and behaviours in $n \mathrm{D}$ systems theory. Multidimens. Systems Signal Process., 11:11-48, 2000.

[54] E. Zerz and V. Lomadze. A constructive solution to interconnection and decomposition problems with multidimensional behaviors. SIAM J. Control Optim., 40:1072-1086, 2001. 
Centre de recherche INRIA Saclay - Île-de-France

Parc Orsay Université - ZAC des Vignes

4, rue Jacques Monod - 91893 Orsay Cedex (France)

Centre de recherche INRIA Bordeaux - Sud Ouest : Domaine Universitaire - 351, cours de la Libération - 33405 Talence Cedex Centre de recherche INRIA Grenoble - Rhône-Alpes : 655, avenue de l'Europe - 38334 Montbonnot Saint-Ismier

Centre de recherche INRIA Lille - Nord Europe : Parc Scientifique de la Haute Borne - 40, avenue Halley - 59650 Villeneuve d'Ascq Centre de recherche INRIA Nancy - Grand Est : LORIA, Technopôle de Nancy-Brabois - Campus scientifique 615, rue du Jardin Botanique - BP 101 - 54602 Villers-lès-Nancy Cedex

Centre de recherche INRIA Paris - Rocquencourt : Domaine de Voluceau - Rocquencourt - BP 105 - 78153 Le Chesnay Cedex

Centre de recherche INRIA Rennes - Bretagne Atlantique : IRISA, Campus universitaire de Beaulieu - 35042 Rennes Cedex

Centre de recherche INRIA Sophia Antipolis - Méditerranée : 2004, route des Lucioles - BP 93 - 06902 Sophia Antipolis Cedex 